Historic, archived document

Do not assume content reflects current scientific knowledge, policies, or practices. 



\title{
BUSINESS CONDITIONS
}

\author{
THESE PRICES \\ SUPERSEDE ALL OTHERS AND ARE SUBJECT \\ TO CHANGE WITHOUT NOTICE.
}

TlkMs. Sixty days net cash to seed Houses of approved credit, except on (wasl

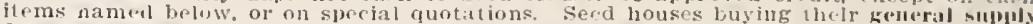
from us will be given June 1st terms. Bills to houses not regularly in the catalugue seed trade are due the first of evcry month following date of purchase.

Grass, l'arm, Bird aud Clover seds, Onian sets, lotatoes, fertilizers, lbone Mills,

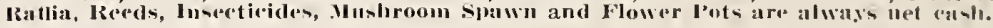

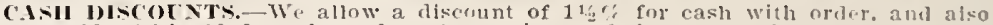
on bills paid whthin 10 days from date ("xccpting cish flums mentioned above).

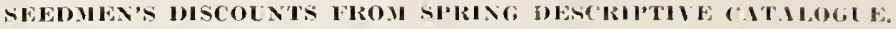

Orders under $\$ 2.00$ for parket secds, $3: 13$ pur cent.

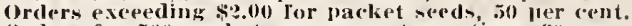

orders for 500 packets or more at wue time, (i) per iont.

Orders for olluces, flints, and (juarta, $331 / 3$ per rent.

Orders fur "Sllecial ofrers" and rul rate "Collections" maly I0 fier fent.

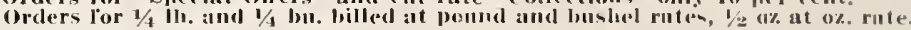

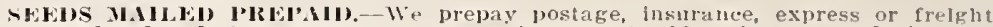
whin :o ordered and 'harge same to customer's account. If package exceeds s.2.0 lil villue it will be sent by insured mail or express and if prepald sucli prepajoment cincliged to eustomer.

Routing Shijments.-Railroad or Express routes given by buyers, whlch should le an each order, will be strictiy followed by us where posslble. In all cases whore the ronte is left to our judgment we will select the best we know, but entirely at tlo risk of the buyer, we assuming no responsibllty for delays in transportation. Please use un every order the following words as routing Instructions: "FIREIGIIT." EXI'IRlix."

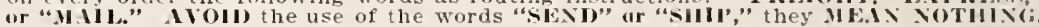

No' W.IRR.INTEl. - We do not warrant in any way, express, or Implied, the contents, or the discription, quality, productiveness, or any other mattcr, of any seeds, bulbs or plants, sold by us, and we will not be in any way responslble for the crop. If the pulchas:-l loes not accept these goods on the above terms, no sale is niad. thereof, and he nusit return them at once, and money will be refunded. Subject in above conditions we make this sale, at the moderate prices at which we sell our goods.

Iauglan's Seed Store (Incorporated).

Eloctrotyes of our cataloguc illustrations can be furnished at $\mathbf{1 5}$ cents per squalc Inch.

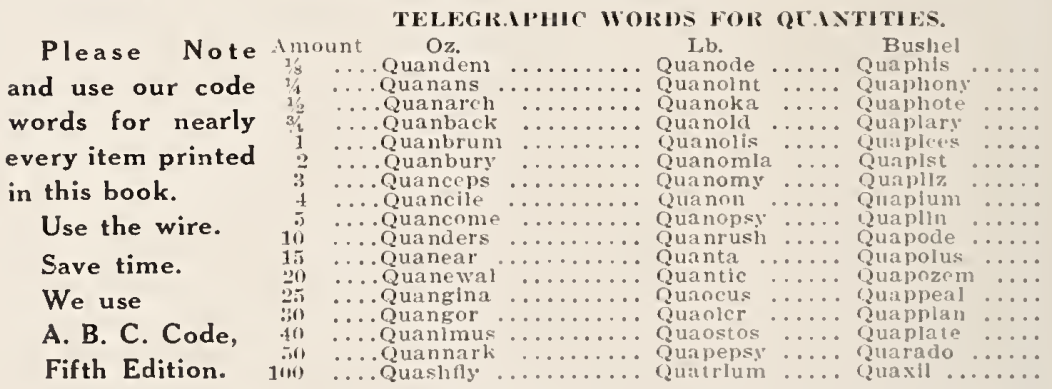

When possilue and so requested we will so far as nur stock permlts luobl thes* prices f. o. h. Xiv Jork Clty, but we do not guarantee to do so, the bulk of our stock being in Chlesgo.

J. C. Vaughan, Pres.

i. H. Vaughan, Vlce-Pres,

C. Cropp, Sec'y \& Treas. 


\title{
Vaughan's Seed Store
}

NEW YORK:

43 BARCLAY STREET

\author{
CHICAGO: \\ $3 i=33 \mathrm{~W}$. RANDOLPH STREET
}

WEST SIDE BRANCH:

803 WEST RANDOLPH STREET.

Greenhouses and Trial Orounds: Western Springs, III.

\section{TRADE LIST OF SEEDS.}

Vaughan's Seeds are the very best that can be had. Our thirty-eight years' experience dealing directly with Market Gardeners and Florists whose livelihood depends upon their growing and producing the highest quality in both vegetable and flowers, is an invaluable aid. We are in constant touch with the largest markets, and the results obtained from our own and other seeds.

Lower prices can be found. However, cheap prices and poor seeds invariably go together. WVe own and grow our seeds at right prices and, quality considered, we can and will meet reliable competition.

Large Dealer's on fill-in orders during the rush season should remember our orders are filled the same day as received. You are assured quick service, high germination, best quality and right prices. Give us a trial. VAUGHAN'S SEED STORE.
ARTICHOKE.
Tel. cipher
Lb. $100 \mathrm{lbs}$

Green Globe............................. . $\$ 4.00$

Tubers (Jerusalem), bu, $\$ 1.25$; bbl. of $23 / 4$ bu., $\$ 3.25$; if shipped from $\mathrm{New}$

York, bu., $\$ 1.50 ;$ bbl., $\$ 3.75 \ldots \ldots$ A batis ....

ASPARAGUS SEED.

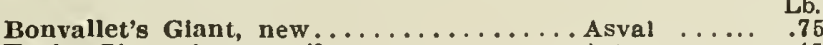

Early Giant Argenteuil.............................. . . . .

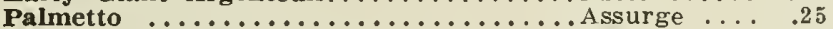

Conover's Colossal........................ 25

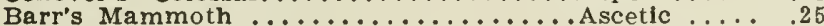

Columblan Mammoth White.......................

ASPARAGUS ROOTS.

Per 100

Per 1000

Bonvallet's Giant, 1-year-old......... Avict . . . \$0.65

Bonvallet's Giant, 2-year-old . . . . . . . . . Avicting. . . 1.00

Bonvallet's Giant, 3 -year-transplants .......... 2.00

Palmetto, 1-year-old ........................40

P'almetto, 2-year-old ........................ .55

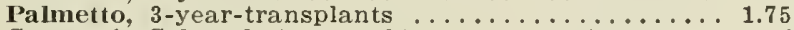

Conover's Colossal, 1-year-old........... Attend ... 40

Conover's Colossal, 2-year-old......... Atfile .... .55

Conover's Colossal, 3-year-transplants .......... 1.75

Colunbian Mammotl White, 2-year-old... Auer ..... . .65

Columbian Mammoth White, 3-year-trans-

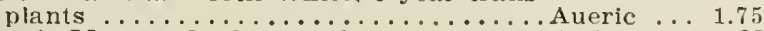

Barr's Mammoth, 2-year-old............ Attic ..... .65

Giant Argenteuil, 2-year-old ...................... 65 


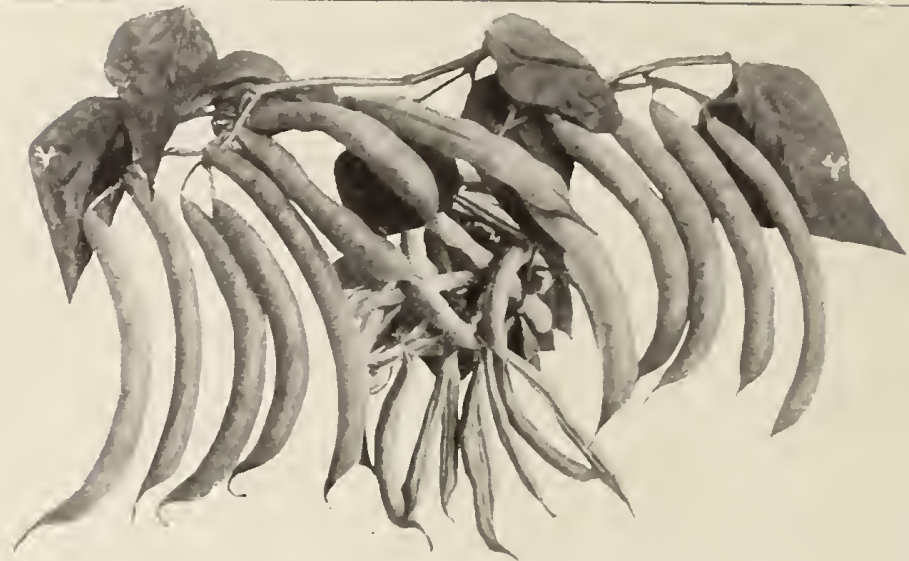

RED VALENTINE-Vaughan's Improved.

\section{BUSH BEANS.}

BEANS ARE VERY SCARCE. AT THE PRICES BELOW WE CAN SUPPLY ONLY IN VERY LIIITED QUANTITY TO OUR REGULAR CUSTOMERS ON A GENERAL ORDER. FOR BEANS ALONF: WRITE FOR PRICES.

GRELN BEANS.

Tel. cipher.

Per Bu.

IRed Valentine, Improved Earliest........ Blacklash ....... \$5.50

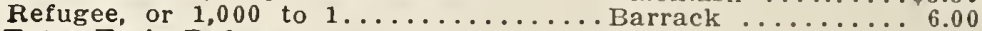

Extra Early Refugee ........................ 6.00

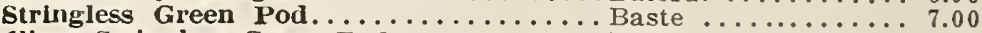

Giant Stringless Green Pod .......... Bash ........... 6.50

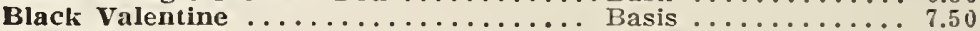

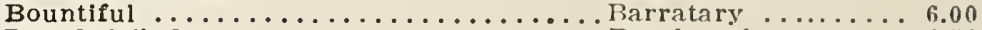

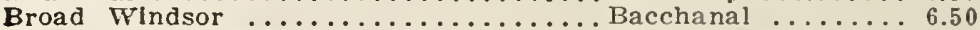

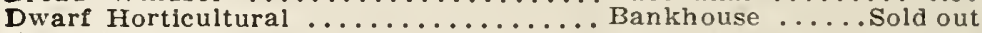

Early Molhawk .......................... 5.50

Early Round Pod Six Weeks.......... Baker .......... 5.50

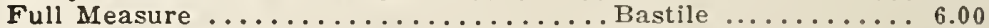

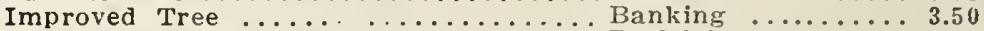

Long Yellow Six Weeks...................... 5.75

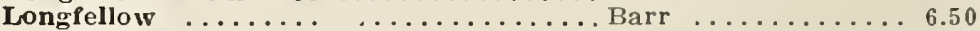

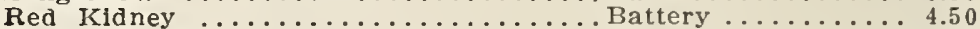

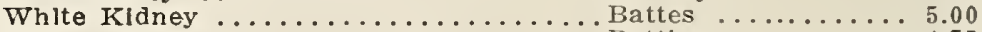

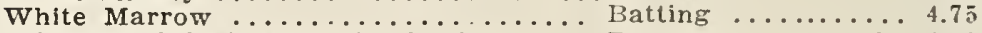

Trlumph of the Frames, for forcing ..... Bassoon ....... pk., 4.50

Stringless Refugee 1,000 to $1 \ldots \ldots \ldots \ldots$ Barut .................. 7.00

WAX BEANS.

Currie's Rust Proof Golden Wax....... Believe ......... 7.50

Round Pod IIIdney Wax (Brittle Wax)... Eibuling ......... Sold out

Improved Dwarf Golden Wax.......... Besee ......... 8.00

Davis Wax, hardiest and most productlve. Bybox ........ 8.00

Wardwell's Early Iildney Wax, one of

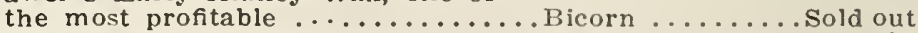

Challenge Rlack Dware Wax .......... Eegrune ....... 8.00

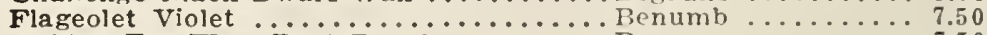

Golden Eye Wax. Rust Proof .................... 7.50

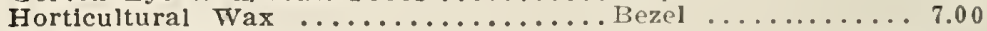

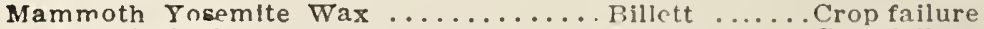

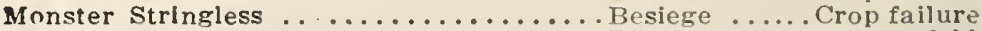

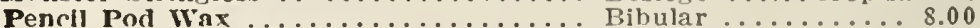

Prollfic German Black Wax .................... 8.00 
IVAX BEANS-Continued.

10 Bush.

Refugee Wax, Keeneys Stringless....... Bewitch ...\$8.50

Per Bu.

Valentine Wax

7.50

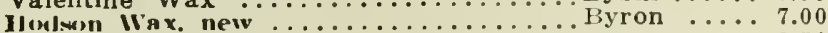

Michigan White Wax............................ . . 8.50

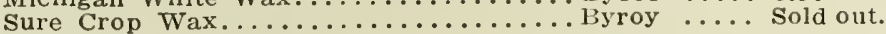

BUSH LIMAS.

New Wonder Bush Lima .................... $7.25 \quad 7.00$

Henderson's Bush Lima ................... Binmack ...6.00 5.75

Burpee's Bush Lima .. . . . . . . . . . . Billow .... 7.00 6.75

Dreer's Bush Lima ........................ . 8.50

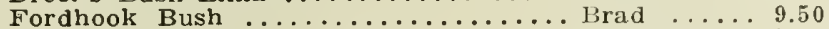

Burpee's Improved ............................ . 9.00

BEANS, POLE.

Early Golden Cluster Wax ............ Bluster ...8.00

Kentucky wonder, or old Homestead.... Bodkin ... 6.25 6.00

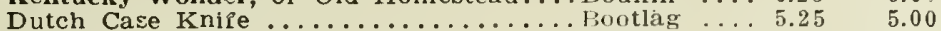

Hortlcultural Cranberry ..................... $5.50 \quad 5.25$

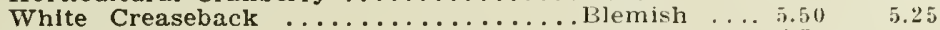

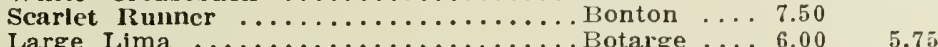

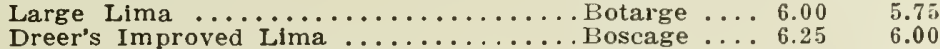

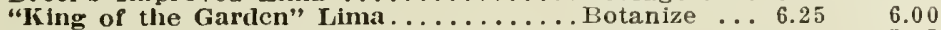

Ford's Mammoth Lima ............................ 6.0

Extra Early Jersey Lima...........................

Slebert's Early Lima ............................ . $6.00 \quad 5.75$

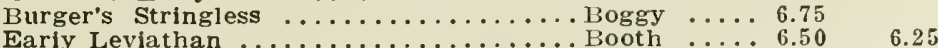

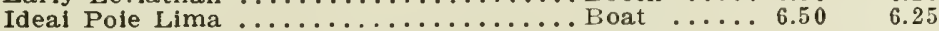

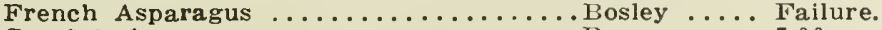

Carpinteria ........................... $7.00 \quad 6.75$

\section{BEET.}

European War conditions make the Beet Seed Market very uncertain. We have had a representative in Europe all this fall and we are prepared to take care of our trade as usual on this and other foreign seed lines. On quantity lots we will be pleased to make special figures.

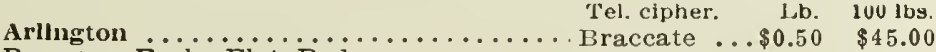

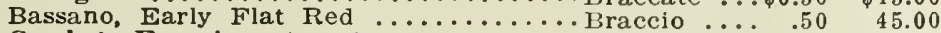

Crosby's Egyptian, American grown...... Brace ..... $.75 \quad 70.00$

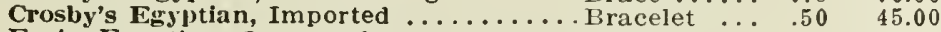

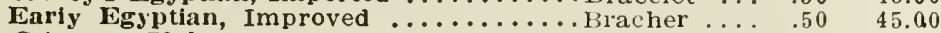

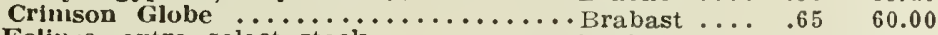

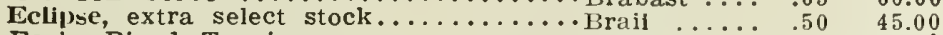

Early Blood Turnip .......................

Dewing's Early Blood Turnip, Sclect.... Bract ...... $50.50 \quad 45.00$

Haif Long Blood, very deep red .......... Brangle ..... .60 55.00

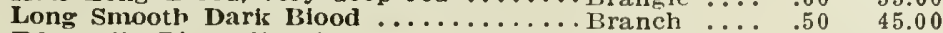

Edmand's Blood Turnip, select stock.... Braja ...... $.50 \quad 45.00$

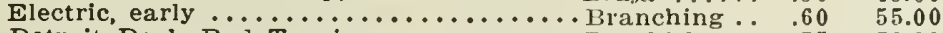

Detroit Dark Red Turnip ................................... 7570.00

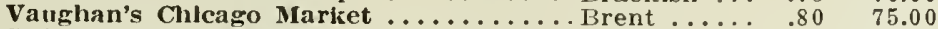

Swiss Chard or Summer Spinach......... Bream .... $.50 \quad 45.00$

Swiss Chard Lucullus ..........................70 65.00

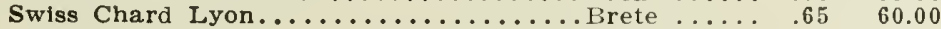

Vaughan's Firebali ......................... .8 $80 \quad 75.00$

Specialties and Novelties.-It is our aim to carry a complete up-todate line. New sorts or special stiains are catalogued as soon as we become convinced of their merits. Oid sorts are dropped when we consider them displaced by improved kinds. For descriptions of latest additions consult the specialty pages of our descriptive retali catalog. 
MANGEL WURZEL.

Tel. elpher.

Lb. $100 \mathrm{lbs}$.

Vaughan's Manmoth Long Red ....... Brigade ...\$ 0.18

Champlon Yellow Globe ................. Breeper .... 18

Golden Tankard ...........................

$\$ 16.00$

16.00

Glant Yellow, Eekendorf .................... 20

16.00

Giant Yellow, Intermedlate ................... 18

Glant Half Sugar Rose........................ 18

18.00

16.00

16.00

Barre's Sludstrup, Danish Grown......... Breeze ..... .22

20.00

SUGAR BEETS.

Vilmorin's Imperial White............ Browning ... .18

16.00

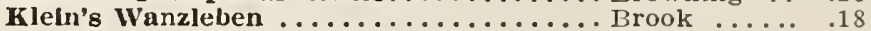

French Red Top ........................... 18

Lane's Improved White .................... 18

16.00

16.00

16.00

BORECOLE Or ITALE.

Vaughan's Exeelsior Mross Curled ...... Bugle ..... .75

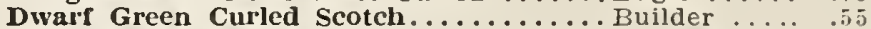

Tall Green Curled Seotch................... . .6urlesque

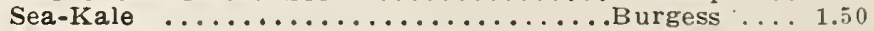

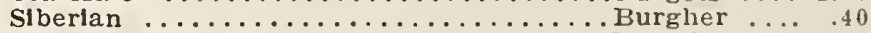

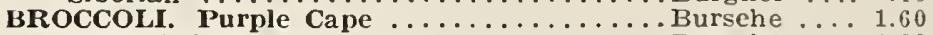

Large White Mammoth .................... 1.60

BRUSSELS SPROUTS, Paris Market........ Bybond ... 1.00

Inıproved Extra Dwarf ................ Bybent . . . 1.00

Danish Imported ........................ 1.50

\section{CABBAGE.}

We eall particular attention to our eabbage seed, all of which is grown in those loealities and countries where the different varieties are ralsed to the best advantage. American varleties, Long Island grown. Seed crop agaln short. Tel. clpher. Lb. 100 IDs.

Vaughau's Sclected Jersey Wakcfield..... Cascane ...\$1.60 \$150.00

Vaughun's Market Gardeners', Vandergaw,

or All Seasons............................. 1.75160 .00

Vaughan's Fremlum Flat Dutch.......................

All Head Early, or Faultless (short erop). Caravas .... 2.25

Autumn King or World Beater ......... Carapace ... 1.60

Bridgeport Drumhead ...............................

Charleston (large) Early ivakefield.......... Chaffer ..... 1.60

Chinese, or Pe-Tsai ...................... 3.00

Danlsh Sunmer Ballhead...................... 1.40

Copenhagen Market (new) .................. 3.50

150.00

125.00

150.00

130.00
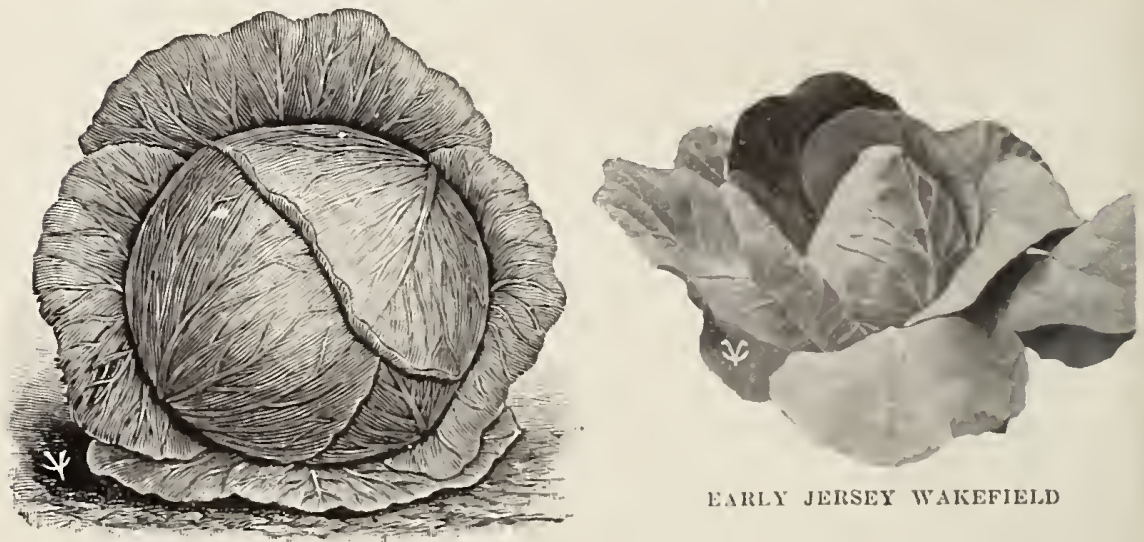

E.ALY JEIRSEY WAKEFIELD

VAUGHAN'S MARKE'T GARUEXERS 
CABBAGE-Continued.

Tel. clpher.

Lb. $100 \mathrm{lbs}$.

Danish Ballhead, Danish grown, tall.... Carcass ...\$1.40 $\$ 130.00$

Danish Balthead, Danish grown, short..... Carden ..... $1.40 \quad 130.00$

Dutch Winter or Hollunder, true stock... Career .... 1.40 130.00

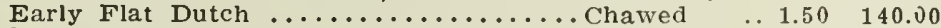

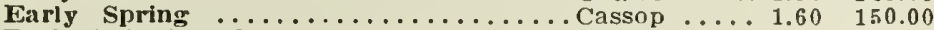

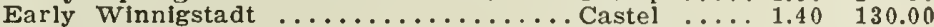

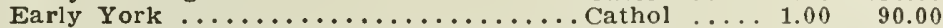

Etampes, Extra Early .............. Carnal .... 1.40 130.00

Express, Extra Early (ready in 90 days). Causer .... . 1.40 130.00

Fottler's Imp. Brunswick Drumhead .... Cedrine .... 1.40 130.00

Glory of Enkhousen ........................ $1.50 \quad 140.00$

Large Late American Drumhead, choicc.. Chancel .... 1.25 115.00

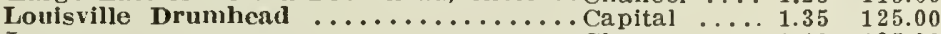

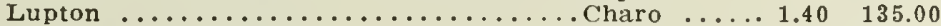

Marblehead Mammoth ............................ 150.00

New Early Flat Hcad ........................ $1.60 \quad 150.00$

New York Early Summer, selected........Cassep ..... 1.75 160.00

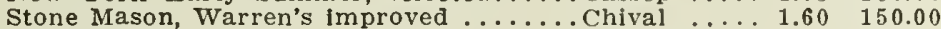

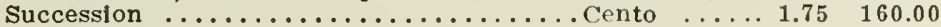

Surchead ................................. 1.75160 .00

SAVOY CABBAGE.

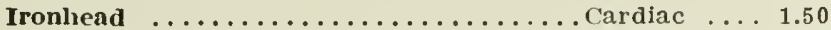

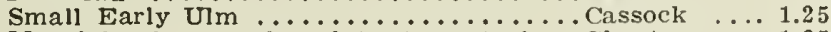

Marvin's, the very best late, true stock... Charter ... 1.25

Improved American ....................... 1.50

RED CABBAGE.

Zenith, new ..........................

Mammoth Toek Red, large, deep red.... Chariot .... 1.75

Extra Early Dark Red Erfurt.................. 1.40

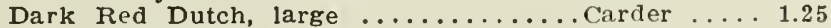

Black Dlamond ........................ 3.00

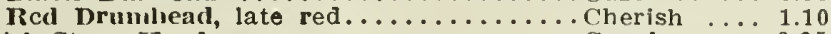

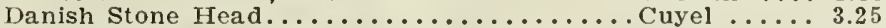

CAlidoON. Targe. smooth .................... 1.25

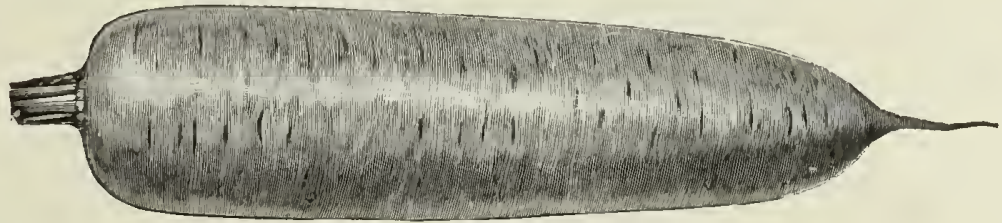

VAUGHAN'S SELECT DANVERS.

\section{CARROT.}

Our Carrot seed is from the most reliable sources, and will satisfy the most critical. Write for prices in quantity.

Inches long. Tel. clpher. Lb. 10 ths

2 Very Early Scarlet (French Horn) .... Clough ... .\$0.80

4 Early Short Stump Rooted......... Clabboste .. .80

6 Nantes, Half Long Scarlet, stump rootclipper .... .65

5 Guerande Half Long (Oxheart)....... Cloan ...... .60

6 Chantenay, one of the best half long... Clabbish ... .70

8 Danvers, (Vaughan's Selcoted Stock)..

only perfect roots................... .90

8 Danvers, Half Long, regular stock.... Classic ..... .90

8 London Market (new) .................... . .5 50

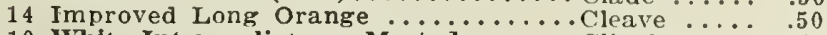

10 Whitc Intermediate or Mastodon ...... Clincher ... .40

16 Long White Belgian, green top ....... Climb .... .35

16 Long Orange Belglan ........................ 


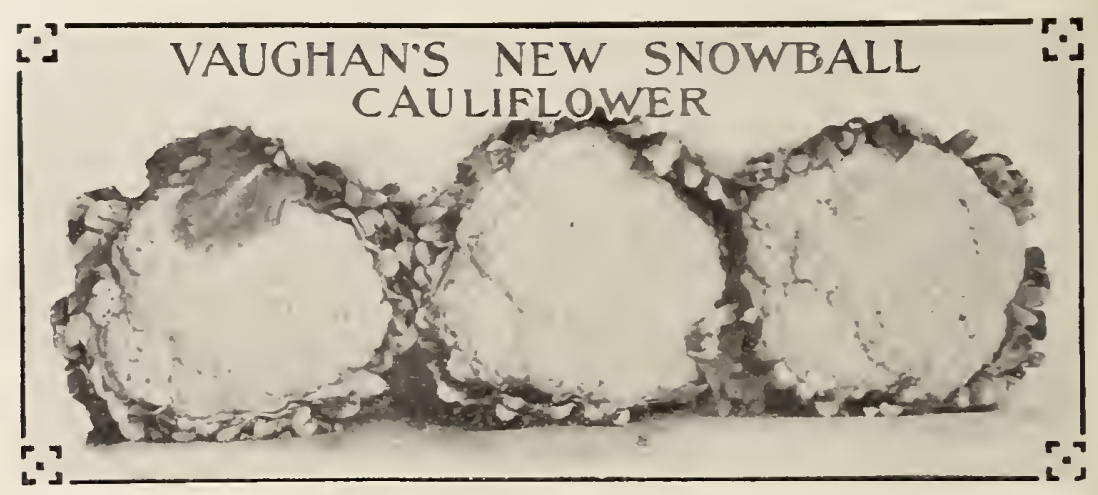

Our stralns are the equal of the world's best products. Prices will be found strletly ln line.

\section{CAULIFLOWER.}

Tel. cipher.

Ox.

Lbs.

Vaughan's New Snowball.......... Cliver ....\$1.75

Vaughan's Earliest Dwarf Erfurt........ Cibospen ... 1.25

Dry Weather .............................. 1.75

Extra Early Dwarf Erfurt ................... . . . . .

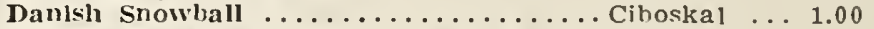

Alglers, large late sort Holland grown. Cibalt ..... .35

Autumn Glant, large and productlve " Clbank ..... .20

Large Early Erfurt. best for late ....... Cicamo .... .50

Lenormand's Shorf Stem Mammoth ...... Cldade ..... .40

Parls, Extra Early ..................... . 25

Parls. Half Early, a standard early sort.. Cicalve .... .25

Earllest of All......................... 1.75

\section{CEIERY.}

Chlcago Glant Self-Blanching (best new

Tel. clpher. Lb. 10 lbs.

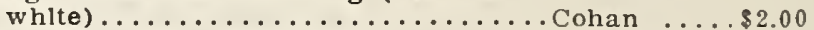

Vaugluan's Glant Golden Heart ...............

Goldien Self- Blanching, French grown ... Coiner ....15.00

Golilen Self-Blanchlng. A merlcan grown . Coining .... 5. 5.00

Glant Pascal, a self-blanchlng varlety.... Cohort .... . . 5

Boston Market ........................... .75

Crawford's Half Dwarf ................ Cockney ... . 5

Dwarf Golden Heart, the old standby .... Cod ...... .75

Evans' Trlumph .......................... . . 75

Far Superlor Many-Heart ....................... .75

Glant White Solld ...........................

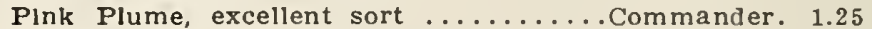

Perfectlon Heartwell .......................... . 80

Perle Le Grand .................. Comming ... .75

Rose Ribbed Parls ........................ 2.00

Schumacher .......................... .80

Solld Ivory or Kalamazno ........... Complle ... 75

White Plume, Vaughan's selected stock... Compound .. 1.40

Winter Queen, hard to beat as a keeper. . Comnaler... 8.85

Soup Celery or Smallage............. Coinst ..... .75

Old Celery Seed, for flavoring ................. . 20

Turnlp Rooted, Dolleatrse ..................... 2.50 
CHERVIL.

Tel. clpher.

Lb.

Double curled ....................... . $\$ 0.50$

CHICORY.

Large rooted Magdeburgh ............ ('ondense ... .75

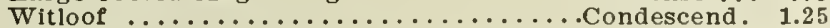

CHIVES, oz., 25c........................ 3.00

COLLAIRIS.

Georgia ................... rondole ... .40

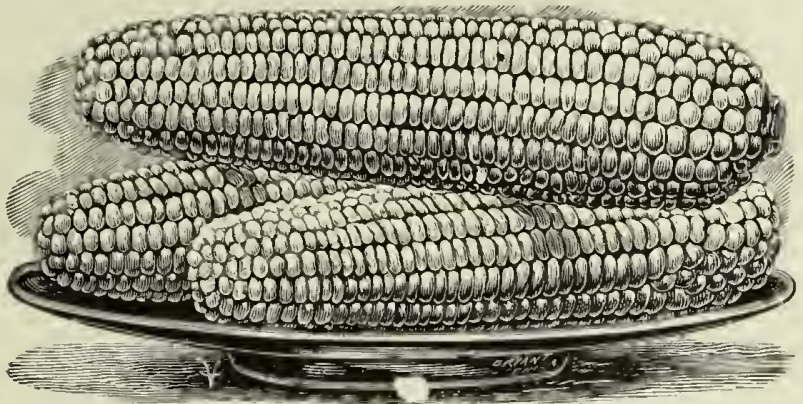

STOWELL'S EVERGREEN.

\section{SWEET CORN}

Our extra early sorts grown on our own Michigan farm. They are much earlier than western seed, truer to type and, due to our curing methods, of higher germination.

EARLY VARIETIES.

Tel. cipher. Bush. In 10 Bu.

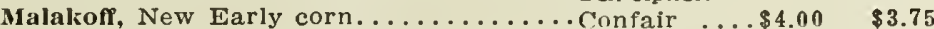

Golden Bantam, New Yellow........................ $4.25 \quad 4.00$

Peep O'Day, very early ............................ $30 \quad 3.25$

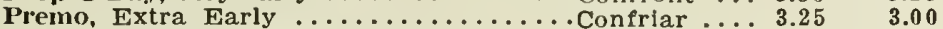

Adams' Early, not sweet but early ....... Confab .... 2.50 2.25

Adams' Extra Early ............................ $2.50 \quad 2.25$

Crosby's Early, an excellent sort ......... Confluent ... $3.25 \quad 3.00$

Chanplon, the earllest large sweetcorn... Confident $\ldots 3.00 \quad \mathbf{2 . 7 5}$

Cory, Mammoth White .............................. 3.00

Early Cory, or First of All............... Connate ... 3.00 2.75

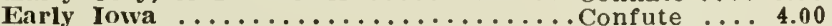

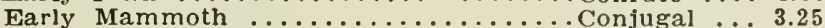

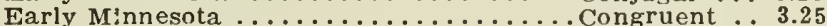

Kendal's Early Giant............... Consummate 3.00

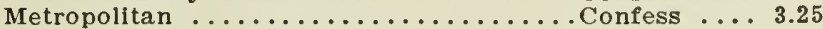

MEDIUM AND LATE VARIETIES.

Bantam Evergreen ....................... 8.50

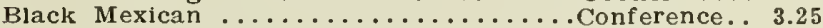

Black Sugar, Vaughan's Eastern grown...Conferring... 3.50

Country Gentleman ........................

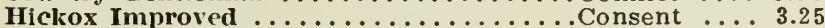

Golden Cream (new)...................... 4.00

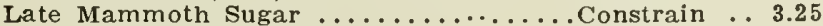

Moore's Early Concord .............................. 3.25

Old Colony, genuine Maine stock................

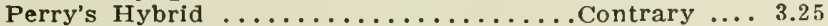

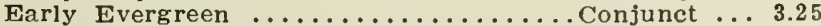

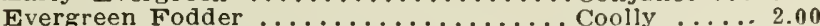

Stowell's Evergreen, Connecticut grown... Contingent , 3.25

Stnwell's Evergreen, Western grown..... Cooler .....3.00

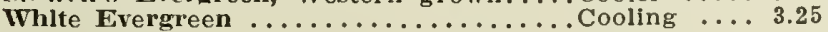

3.00

3.00

2.75

3.00

7.50

3.00

3.25

3.25

3.00

3.00

3.00

3.00

3.00

3.00

1.75

3.00

2.75

3.00

CORN SALAD.

lb. $10 \mathrm{lba}$

Round-Leaved, large seeded ...........Conductive... $0.40 \quad 3.50$ 
Tel. clpher.

Lb. 10 Ibs.

CRESS, CURLED GARDEN ............ Cuniform .. \$0.25

$\$ 2.25$

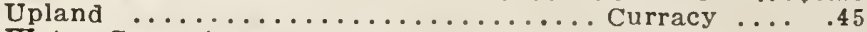

Water Cress, true ........................ 300

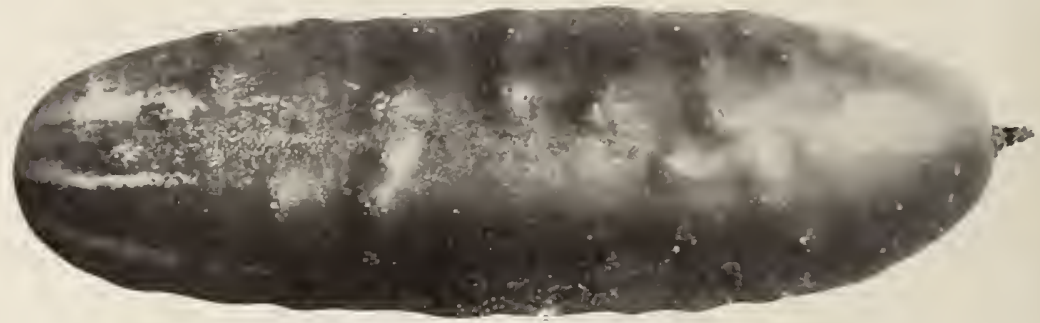

VAUGHAN'S ARLINGTON WHITE SPINE.

CUCUMBER.

Tel. cloher.

Lb. $100 \mathrm{lbs}$.

Arllngton White Spine (Vaughan's Imp.) .. Creduling . $\$ 0.55$

Metcalf's White Spine (Fancy Arlington). Cyrus ..... .55

Westerfield Chicago Pickle, true........ Croft ..... .45

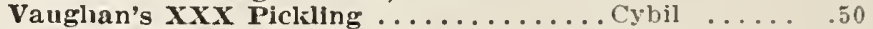

Jersey Plckling ............................ 45

Snow's Fancy Picliling ...................... .50

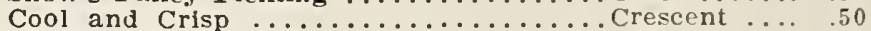

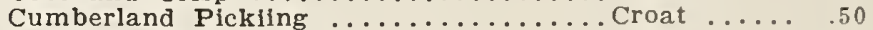

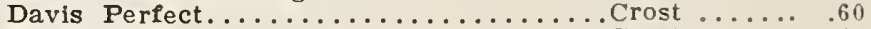

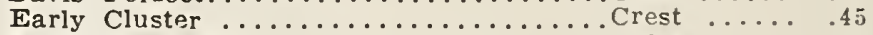

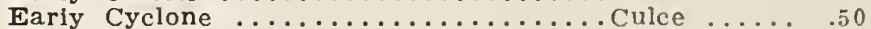

Early Frame ............................ 45

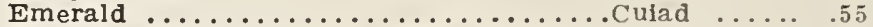

Evergreen, or Extra Long White Spine...Crimpie ... .50

Extra Early Green Prolific ............ Crimson .... .50

Fordhook Famous ...................... 5 . 55

Fordhook Pickling ....................45

Green Prolific or Boston Plckilng....... Croaket .... .45

Improved Long Green, extra good strain. . Croon ..... . .55

Improved White Spine .....................45

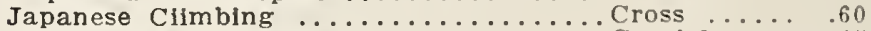

Nlchol's Medium Green ........................45

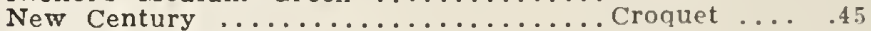

Rockyford (Klondyke) ............... Crowbar . .. .55

West India Gherkin............................ 1.00

Carter's Model, for forcing, 1 oz., $\$ 6.00 \ldots$ Cubical ...

Noa's Forcing, very prolific, $1 / 4$ oz., $75 \mathrm{c}$. Cully ......

Prescott Wonder, 1/4 oz., $\$ 1.50 \ldots \ldots \ldots$ Cuiprit ....

Rollison's Telegraph. 1/4 oz., $\$ 1.75 \ldots \ldots$. Culpably....

Vaughan's Prolific Forcing, $1 / 4$ oz., \$1.00. Culvert ....

D.INDELION.-Thick Leaved or Cabbaging. . Dan ..... 3.75

ENDIVE, Broad-lenved White.......... Elastic .... .60

$10 \mathrm{lbs}$

Ever-White Curled .......................... 60

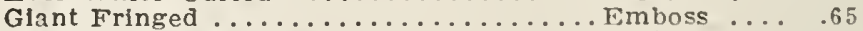

Green Curled summer ..................... .60

Green Curled Winter............................60

Moss Curled, very fine..............Empress ... .65

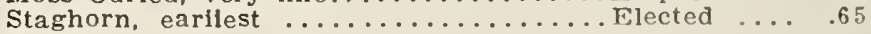

$\$ 50.00$

50.00

40.00

45.00

40.00

45.00

45.00

45.00

55.00

40.00

45.00

40.00

50.00

45.00

45.00

50.00

40.00

40.00

50.00

40.00

55.00

40.00

40.00

50.00 


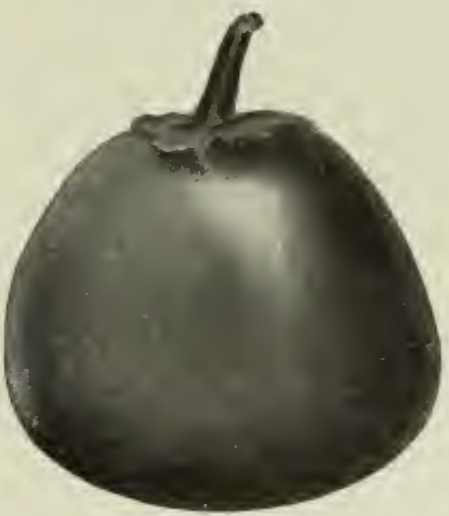

NEW YORK IMPROVED SPINELESS.

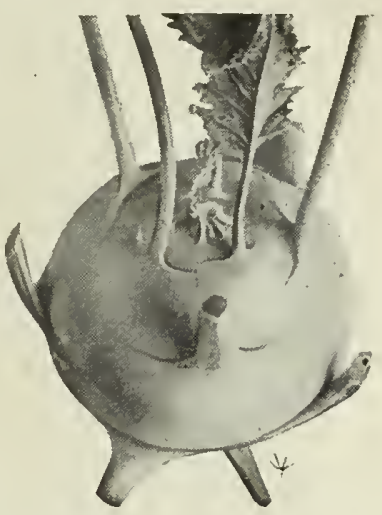

SHORT-LEAVED EARLY ERFURT KOHLRABI.

EGg PLANT.

Tel. cipher. Lb.

Very Early Dwarf ............... Education ...\$2.00

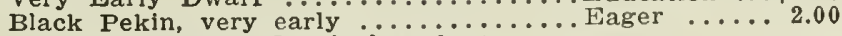

New York Improved Spineless, best......

New Jersey gardeners' selection ........ Edda ..... 3.00

Black Beauty, new ............................... 30

GARLIC.-Sound dry bulbs. Write for prices.. Gabel ......

HORSE RADISH.-Horse Radish Sets, $100,60 \mathrm{c} ; 500, \$ 2.75 ; 1,000, \$ 5$. New Bohemian, per $100, \$ 1.00$; per 1,000 . $\$ 7.00$.

\section{IIERBS.}

Tel. ciph. oz. $1 \mathrm{~b}$.

Anise ......... Habitat.. $\$ .05 \$ .50$

Balm ..........Hackel. .12 1.50

Bene ...........Halcyon. .05 .50

Borage ........Halser... .05 .50

Coriander ........Handy... .05 .40

Caraway ........Halter....05 .20

Catnip...........Halyard. $.25 \quad 3.00$

Chamomile .......Humel.. $35 \quad 3.00$

Dill, 10 lbs., \$3.00.Harbin.. .05 .35

Fennel, Florence..Hardich. $.10 \quad .60$

Fennel .........Hardness .05 .40

Henbane ..........Hatred...10

Hop ..............Hauter...50

Horehound .....Haven. ... 101.25

Hyssop .........Havoc.... 10101.20

Lavender Splca.. . Hawser. . $.10 \quad .75$

Peppermlnt, per 100 seeds, 15 c.; per 1000 seeds, $\$ 1.20$. Help.....

KOHLRABI.

Short-leaved Early Erfurt, best strain...Knitter ...\$1.65

Klng of the Earlles ........................... 1.75

Early White Vlenna ..............Kolpie ....\$1.00

Early Purple Vienna .............. Keblap .... 1.10

LEEK. Broad Scotch .......................... $\$ 0.75$

Large Musselburg, of enormous size..... Lactic ..... .75

Rouen, Large Winter ....................... .75

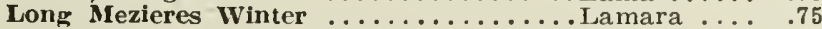

Bulgarian .............................. 1.50
$\$ 90.00$

100.00 


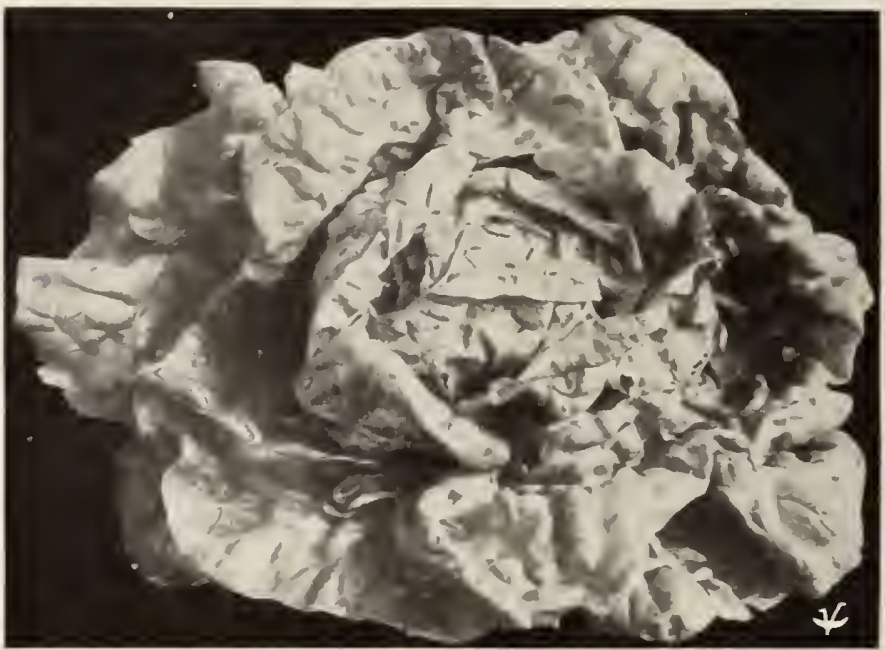

VAUGHAN'S ALL SEASUNS.

LE'T'TUCE.

CURLED VARIETIES. Tel. cipher.

T.h. 100 ths

Blacl-Seeded Simpson, very choice...... Landman ..\$0.50 \$45.00

Blacir-Seeded Simpson, selected stock.... Landing ... $.55 \quad 50.00$

Grand Rapids Forcing .............................50 45.00

Grand Rapids Forclng, select.........................6er ....

Eariy Curied Silesia ...........................45

Eariy Curled Simpson .......................45

The Morse ............................. .50

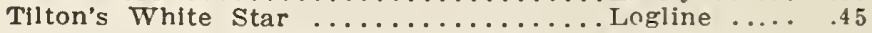

CABBAGE AND HEAD VARIETIES.

All Seasons, Vaughan's .......................... .55

$.55 \quad 50.00$

May King, very early........................65

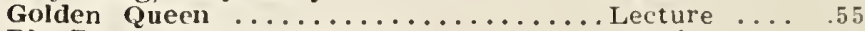

Blg Boston, extra selected ........................ 60

Black Seeded Tennis Bali ............................. 45

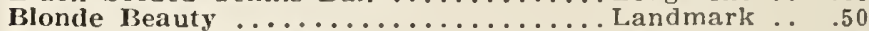

Brown Duteh Cabbage ................................... 45

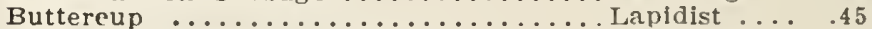

California Cream Butter ............................ 50

Deacon, heads large and solld ................. .50

Denver Market Forcing ................... .55

Glant Crystal Head ............................. 60

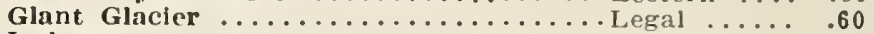

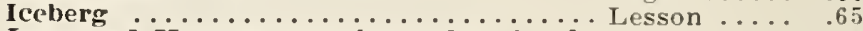

Improved Hanson, very large firm heads.Levant .... .55

Mammoth Black-Seeded Butter ........Lintman ... .50

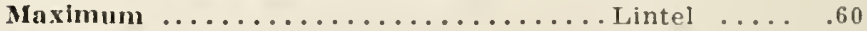

New York ............................. . . . 0

Perpignan, or Defiance Summer................. . . 50

Prizehead .................................... 50

St. Louis Butterhead, very fancy strain... Livery .... . .50

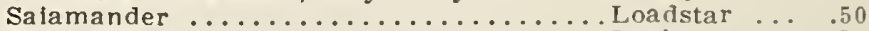

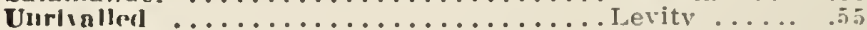

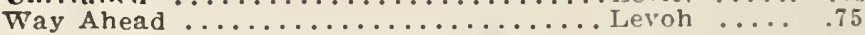

White Cabbage. good head lettuce....... Luxury .... .55

Tennis Rall White Seed (Boston Market).Luthern .... .50

Paris White Cos...............................50

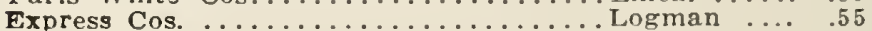

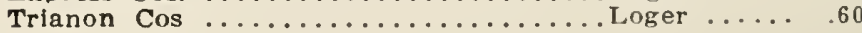

55.00

40.00

40.00

45.00

40.00

50.00
60.00

50.00

55.00

40.00

45.00

40.00

40.00

45.00

45.00

50.00

60.00

50.00

45.00

55.00

65.00

45.00

45.00

45.00

45.00

50.00

50.00

45.00

45.00

50.00

55.00 


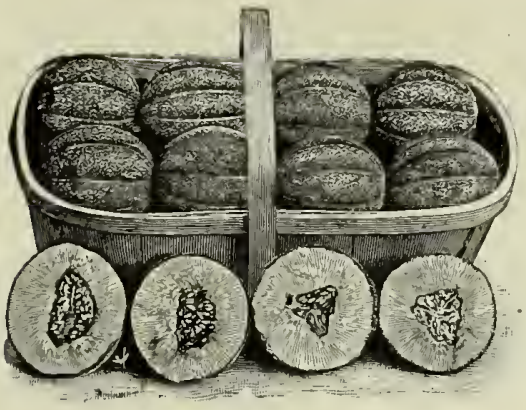

PAUL ROSE.

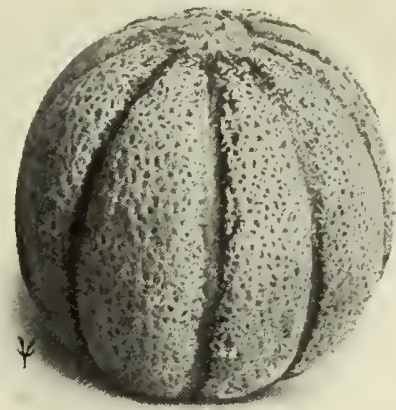

HOCLUO.

\section{MUSK MELON.}

Our Musk Melon Seed is raised in districts where the different varieties are at their best and produce ideal melons of their respective types.

Tel. cipher.

Lb. $100 \mathrm{lbs}$

HOODOO. Introducer's Strain........ Mazin ....\$1.50

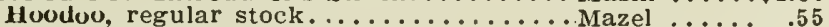

PAUL ROsE. (Petoskey.)

50.00

Vaughan's Select Stock ...........Maxmillian.. 1.50

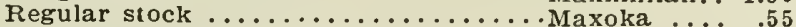

THE OSAGE, Truc ..............

Stock Seed (Mlchigan grown)........Mayport ... 1.40

Regular Stock, ralsed from stock seed.. Maypole $\ldots . .60$

Chicayo Marliet Nutueg ....................... .65ing

Netted Gem, round, choice stock..........Malignent $\ldots . .45$

Netted Gem, oval, choice stock.................. .45

Netted Gem Rocky Ford, choice........ Makkat .... .50

Netted Gem lRocky Ford, select.................... 1.25

Emerald Geni, one of the sweetest..... Mallard .... .60

Tip Top. a very fine market melon.........Melody ....

Extra Early Nutmeg or Early Cltron.... Mamma .... .50

Chanipion Market ........................

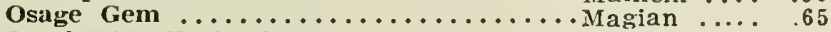

Landreth's Early Citron ....................

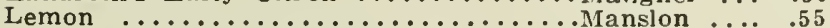

Improved Jenny Lind, selected seed ..... Marker ..... .55

Hackensack .............................. .50

Extra Early Hackensack, best straln..... Maniple .... .50

Montreal Market Nutmeg, very large.... Matry ...... .65

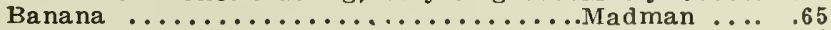

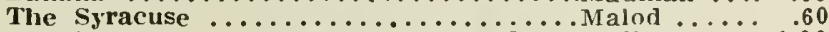

Vaughan's Improved Milwauke Market. Malison .... 1.00

MANGO MELON, or Vegetable Orange.......Mortar .... .75

MARTYNIA Proboseldea ...................... 1.25

\section{MUSTARD.}

White London .........................

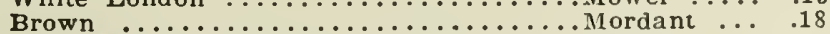

Chineas. or Giant Southern, curled......Morat .... .20

Fordhook Fancy ....................... 20 


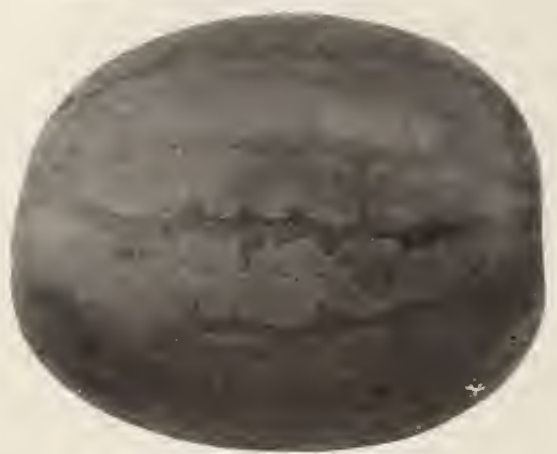

CUIE"X゙ HEXT V.IRTI WATERMELON

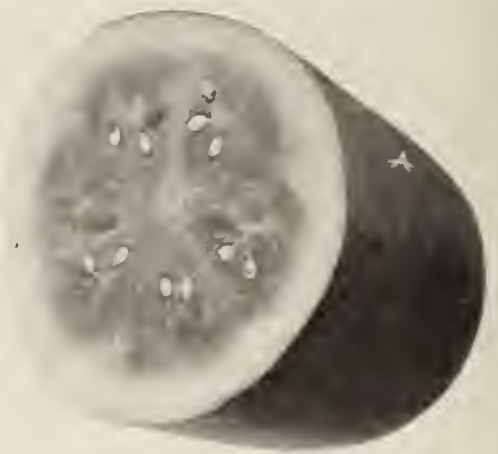

TOM TATSON

\section{WATERMELON.}

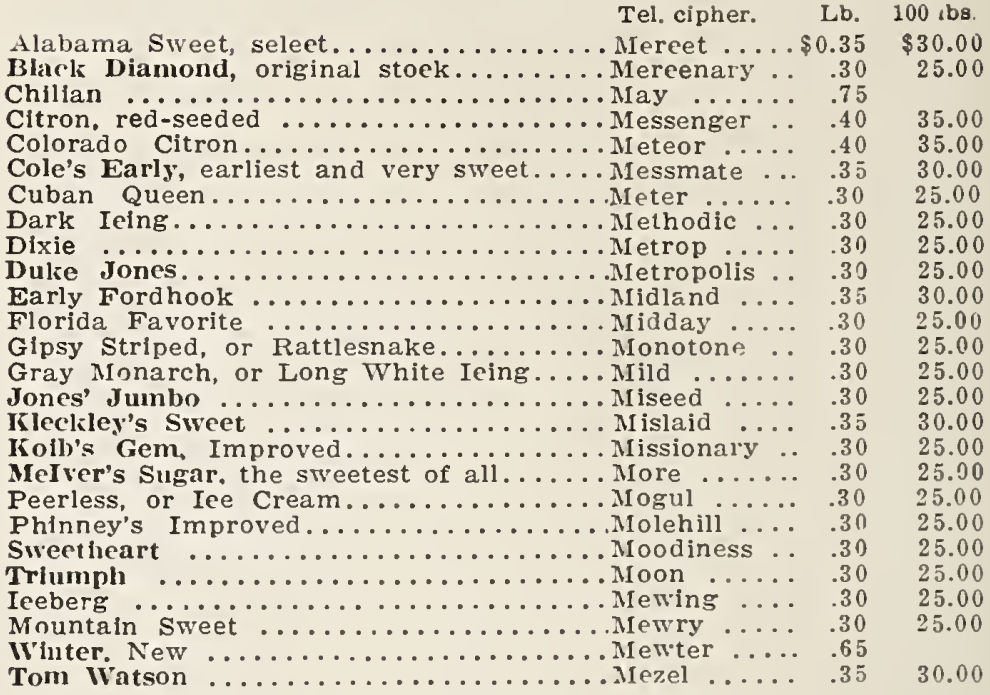

\section{MESHIROOM SPIWX.}

Write for quotation on 1,000 lbs, and upwards. We reeeive from 6 to $\mathrm{S}$ importations a year.

Pure Cultue Mmerican Spawn.

Standard Bi.ick, all varieties, per brick, $20 \mathrm{c}, 10(1 \mathrm{bricks}, \$ 10.00$. f. o. b. Chicago; f. o. b. New York, 100 bricks, $\$ 10.50 \ldots \ldots \ldots$. . . .

Dilcet Briek, all varieties, per bricl, $25 \mathrm{e}$, 100 brieks, $\$ 13.00$, f.o.b. Chieago; f. o. b. New Tork, 100 brielis, $\$ 13.50 \ldots . .$. Mummer

English, per lb., 12c; 10 lbs., $\$ 1.00 ; 100$ lbs., $\$ 6.50 ; 1,000$ lbs., $\$ 60.00$

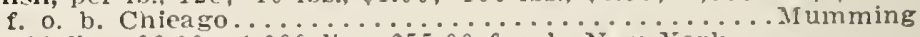
100 lbs., $\$ 6.00: 1.000$ lbs., $\$ 55.00$ f. o. b. Nem York. 


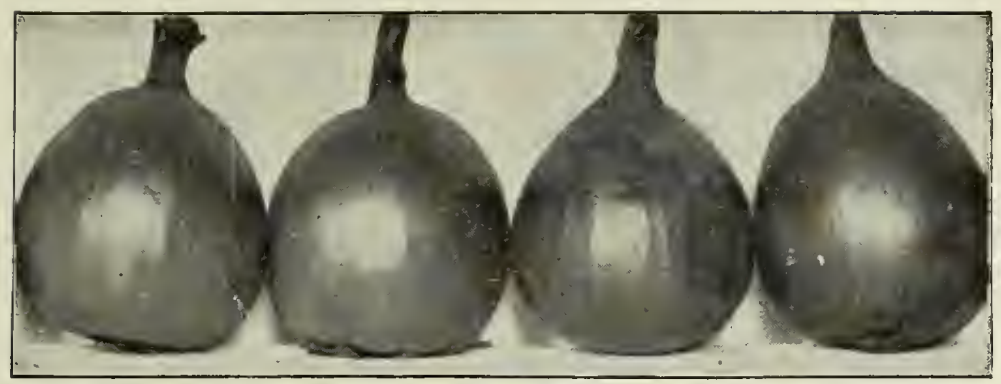

VAUGIIAN'S SELECT OIIIO YELLOW GLOBE. ONION.

ONIONS. Write for speclal quantity prices. Tel. cipher. L.b. 100 lbs.

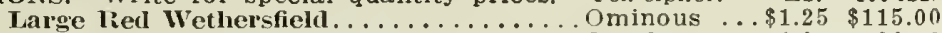
Soutliport Large Red Giobe. . . . . . . . . . . . . O racle . . . $1.00 \quad 90.00$ Southport Large Iied Globe, Eästern. . . . . . Obvious . . . . 1.35 125.00 Southport Large Yellow Globe............ Oral . . . . . $1.00 \quad 90.00$ Southport Large Yellow GIobe, Eastern. . . Owcho ..... 1.35 125.00 Sonthport White Giobe ....................... 1.75 165.00 Ideal White Globe, Eastern grown......... Ordanox ... 2.75

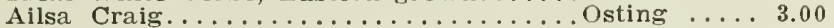

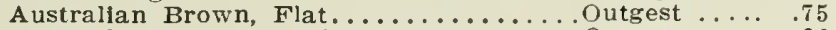
A ustrallan Brown, Globe shaped........ Overum .... .90 Bernuda White (True Teneriffe) ....... Ouger .... 1.75

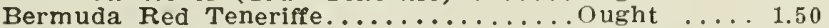

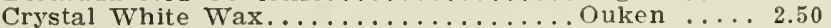

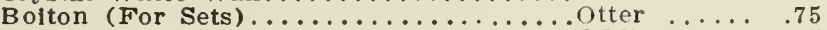
Extra Early Flat Red, Eastern grown..... Oddity ..... 1.15

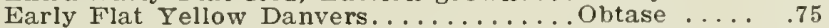

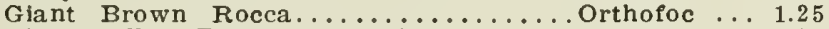
Giant Yellow Rocca or Spanish King..... Orstock ... 1.25

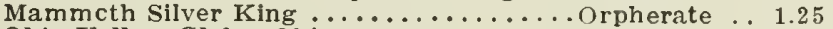
Ohio Yellow Globe, Ohio grown......... Ohope .... 1.50

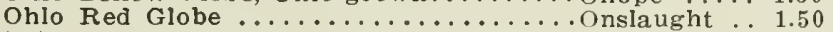
Prizetaker or Mammoth Yellow Spanlsh. Oppose .... .90 Vaughan's "Apple Shaped" Red Globe... . Obliging . . . 1.35

Vaughan's Piekling............................. 1.50

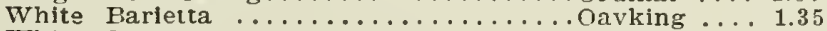

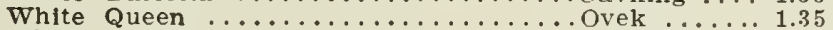
White Portugal or Silver Skin ........... . . . . . . . . . . . . 1.25

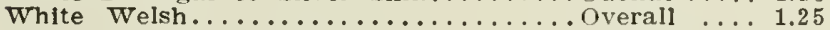
Yellow Cracker, very choice............. . . . . . . . . 0

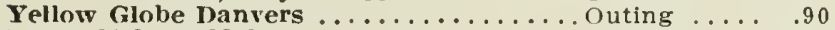

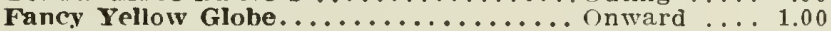
Yellow Strasburg or Yellow Dutch........ Outil ..... .75

275.00 65.00 80.00 150.00 125.00

65.00 105.00 65.00 110.00 110.00 110.00 140.00 140.00 80.00 125.00 140.00 125.00 125.00 115.00

75.00 80.00 90.00 65.00 ONION SETS.

We mall samples and quote lowest prlces on application.

White Multiplier Sets..... Owaken. White Bottom Sets........ Owack. Yellow Bottom Sets........ Owaf. Red Bottom Sets........ Owaking.

Rbls. extra, $25 \mathrm{c}$. Bags at cost. OKIRA.

White Velvet ................. Oblate ... $\$ 0.20$

Dware $\ldots \ldots \ldots \ldots \ldots \ldots \ldots \ldots \ldots$. . . . . . . . . . . . . . . . . . .

Tall, Perkin's Mammoth................... 20
Owabut.

Egyptian, winter top sets... Ovupt. Red Summer top sets...... O Owank. Yellow Potato ........... Ollace.

Lb. $100 \mathrm{lbs}$. $\$ 18.00$ 18.00 18.00 


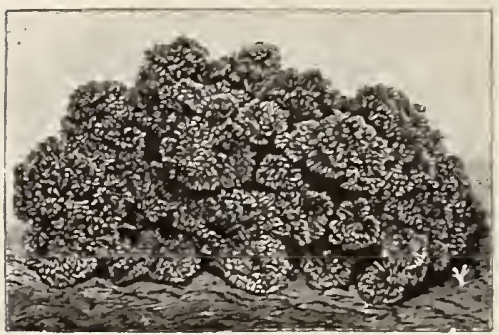

VAUGHAN'S CHAMPION MOSS CURLED PARSLEY.

PARSLEY.

Vaughan's Champion Moss Curled.

Double Curled

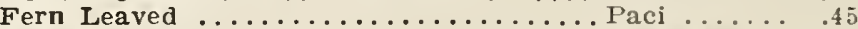

Plaln ...................................40

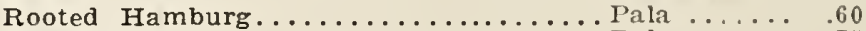

Vaughan's $\mathbf{x x} \ldots \ldots \ldots \ldots \ldots \ldots \ldots$. . . . . . . . . 75

I'IRSNTP.

Long Smooth, or Abbott's Hollow Crown. Palmpaid ... .25

Improved Guernsey, smooth, fine grain... Pallor ..... .25

Magnum Bonum........................... .25ara

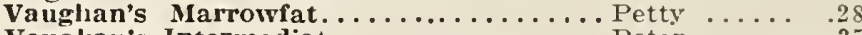

Vaughau's Intermediate $\ldots \ldots \ldots \ldots \ldots$. . Peter ..... 35

\section{PEAS.}

22.00

22.00

22.00

25.00

30.00

Bush.

There are no better stocks of peas. Many varieties are grown from our own tested stock seeds.

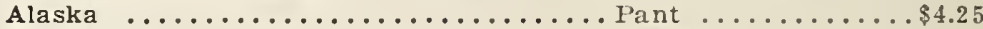

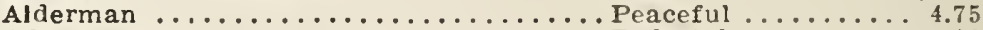

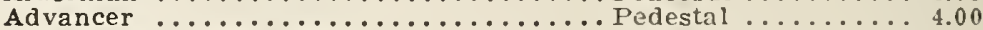

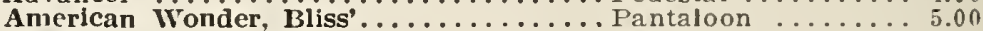

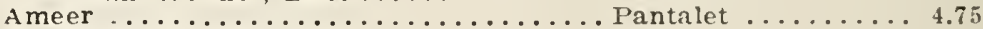

Black Eyed Marrowfat..................... Pantomime ..... 40

Champion of England.............. Parent ........ 4.50

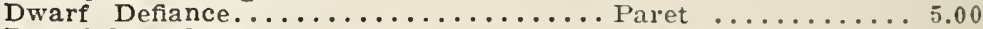

Dwarf Grey Sugar................ Perigraph ........ 6.00

Dwarf Telephone, or Daisy (extra choice). Pemphone ....... 5.00

Extra Early Premium Gem, or Little Gem. Passport . . . . . . . 4.75

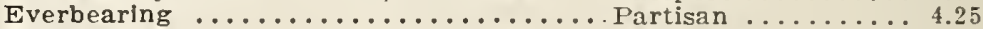

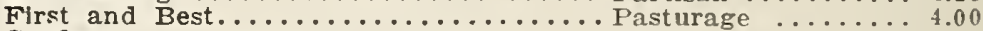

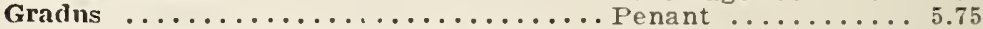

Herolne, one of the best Second Earlies... Paver .......... 4.75

Horsford's Marlet Garden........... Pavillion ........ 4.00

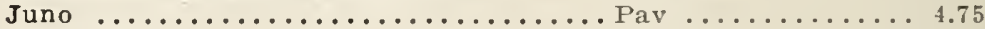

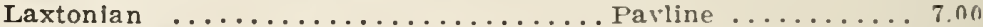

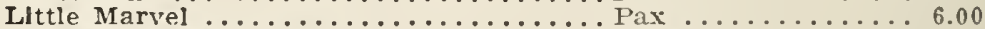

Long Island Mammoth .............. Pedantry ....... 4.50

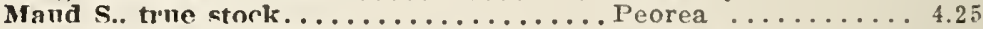

Mammoth Podded Sugar .................... 6.50

Nott's Excelstor, one of the best earlles... Pella ......... 5.00

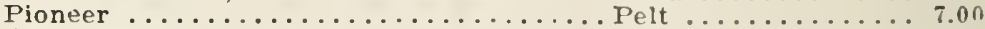

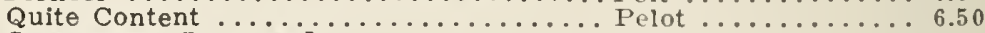

Stratagem. Inproved............. Percolate . . . . . 5.00

Surprlse, fine extra early........... Penult ........ 5.00

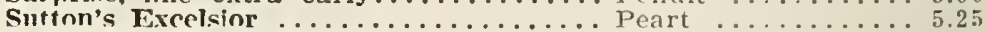

Trlenhone, regular stock................ Perfidius ....... 4.50

Telrnhone. Vaughan's Improved....... Perfid ......... 4.75

Thomas Laxton.................. Pealing ........ 5.75

Vauchnn's Swcet Market...................... 5.50

Velncity ..................... Pezel .......... 5.00

White Marrowfat, large.............. Perk .......... 4.00

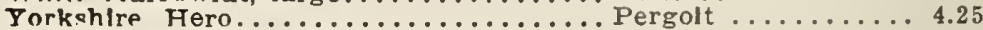

Canada Field, Closest market prlce..... 


\section{PEPPER.}

'Tel. clpher.

Magnum Dulce, the largest.......... Perm . . . \$4.50

Vauginan's Improved Sweet Mountain.....Picaroon ...2.00

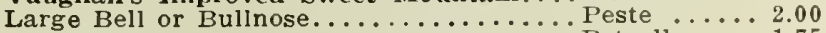

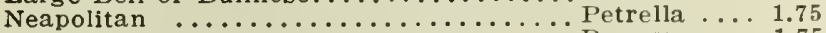

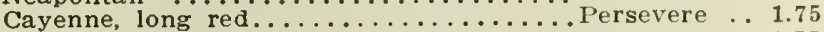

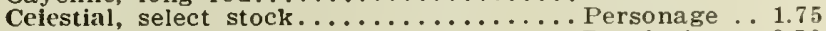

Chinese Giant .......................... 3.50

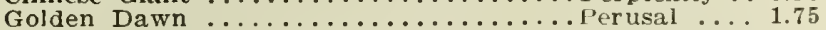

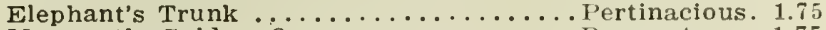

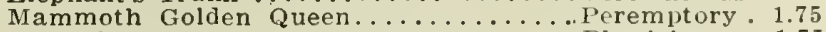

Red Cherry.................. Physician . 1.75

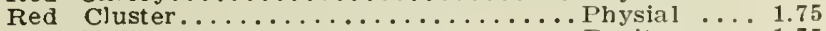

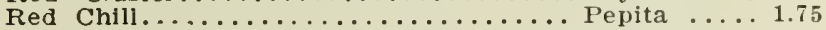

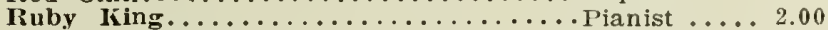

Spanish Monstrous .............. Pibroch ... 1.75

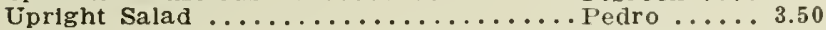

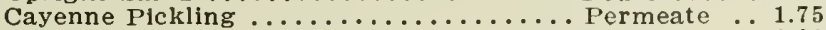

Tabasco ................................. 3.00

SIVEET POTATOES. ( 3 barrel lots, $25 \mathrm{c}$. less).

Prices subject to change. Quotations given.

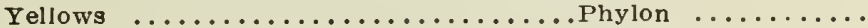

Reds, wlll be scarce agaln ............. Phyllls ...........

The African Bunch Yam (Vineless) ..... Phylas ............

SEED POTATOES.-Northern Grown. Prices subject to change.

Our potatoes are grown especially for us from our stock seed under contract, and are true to namc, selected as to size and shape. Barrel contains 11 pecks. (No charge for bbls.) We have a good stock of the following which we own at right prices. Prices vary with the market. We will quote by mall upon request.

Vaughan's White Ohlo, early...........Plnnac ....

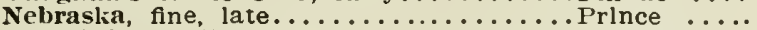

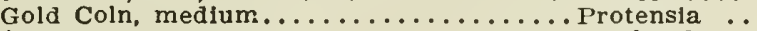

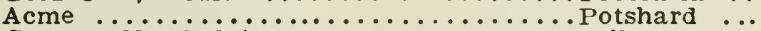

Carman No. 3, late........................

Irlsh Cobbler, good, early..........................

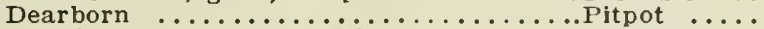

Vauglian's Improved Eariy ohio, fancy.. Pinnacle ...

Rural New Yorker No. 2, late.......... Plexus .....

Slr Walter Ralelgh, late............. Raleigh ...

Woodbury White Rose.........................

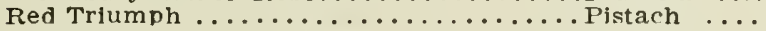

Russet Rural or Petoskey.............. Pitash ....

\section{PUMPIKIN.}

Lb. $100 \mathrm{lbs}$.

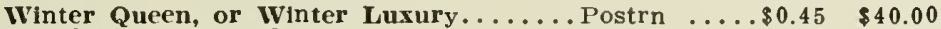

Vaughan's Mammoth Prize..................... $70 \quad 65.00$

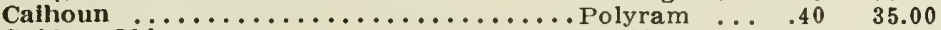

Golden obiong........................ $40 \quad 35.00$

Japanese Ple............................. $50 \quad 45.00$

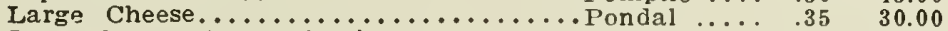

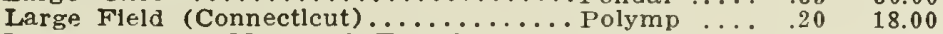

Large Tours, or Mammoth French...... Ponert .... .45 40.00

Quaker Ple.............................. .45 40.00

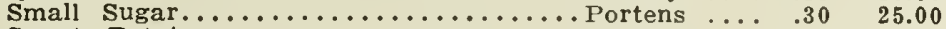

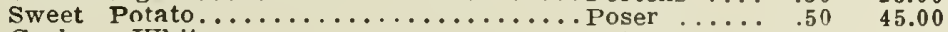

Cushaw, White ..........................

Cushaw, Striped .......................6oted ....

\section{RHUR.ARI3.}

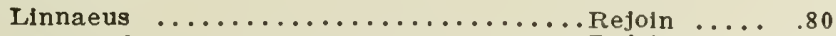

Mammoth ............................ Rejoire ... 80

75.00

RoOT'S. We have a fine stock. Write for speclai prices.

Electrotypes of our catalog illustrations can be furnished at $15 \mathrm{c}$ per sq. in. 


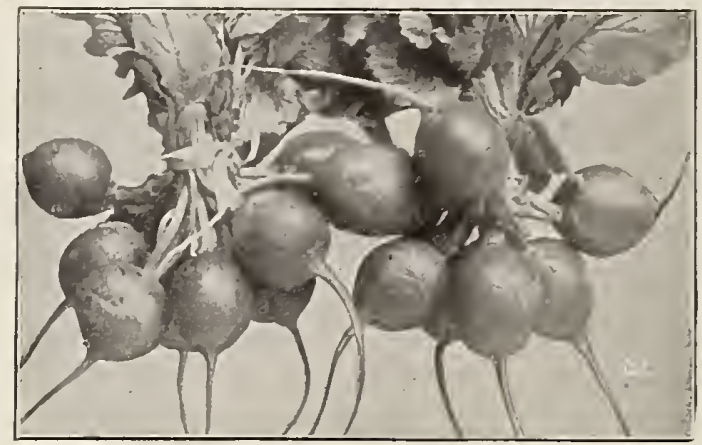

VAUGHAN'S SCARLE"T CILOBE.

In comparing prices customers must bear in mind that our Radish Seed is raised from selected transplanted roots.

RADISH.

All Seasons ................. Rabble ...\$0.35

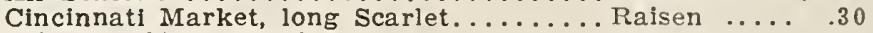

Crimson Giant Foroing.............. Radiate .... 440

Earliest Carmine Turnip, Vaughan's.... Ramoose ... .35

Early Scarlet Turnip, White Tip Forcing. Ranter .... .35

Earliest White Olive-shaped..........Radix ..... .40

Earliest White Turnip (short leaved).... Raven .... .35

Early Scarlet Globe, Vaughan's Select... Ratsbane ... .40

Early Scarlet Giobe, Ordinary.........Ratooner ... .30

Early Deep Scarlet Turnip...........Rancer .... .32

Early Scarlet Globe, White Tip Forcing

(Scarlet Turnip, White Tip Forcing) . Reggy ..... .40

Early Long Scarlet (short top)........ Rapacious .. .30

Eariy White Giant Stuttgart.......... Reform ... . .35

French Breakfast ..................... . . . . .

French Breakfast, Improved........... Readjust ... . .40

Vaughan's Giant Butter Radish (new) ... Reade ..... . .55

Golden Yellow Summer.............. Raysop .... .35

Half Long Deep Scarlet............. Reach ..... .35

Improved Chartier ............... Reading … .35

Long Brlghtest Scarlet, white tip (Cardinal) Realley .... .30

Long IVhite Strassburg............. Reaping ... . 30

Long White Vienna or Lady Finger.... Reapting ... .35

Non Plus Ultra......................... . . 35

Olive Deep Scarlet Short Top (20 days)

forcing.............................. .45

Olive Shaped Deep Scarlet..........Rebuke .... .40

Oval Yellow May ................. Redeam ... .35

Scarlet Turnip, White Tip (select)...... Reclaim .... .32

Triumph, scar!et and white ......... Reanol ..... .40

Vaughan's Market, long white........ Rand ..... 35

Vaughan's Cardinal Forcing.........Random .... .45

Wood's Early Frame...............Regrste .... . 30

White Icicle, the earliest long white..... Reacting $\ldots . .35$

IVINTER VARIETIES.

Chinese Mammoth or Celestial....... Raiment .... .40

California Mammoth White Winter...... Regan ...... .40

Half Long Black Winter............ Rent ...... .40

Long White Spanish..........................40

Long Black Spanish................ Reality .... .40

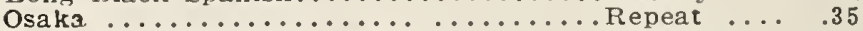

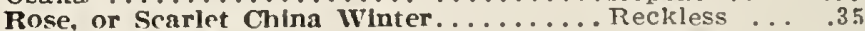

Round Black Spanish..........................40

Munich .....................Ranting ... 40

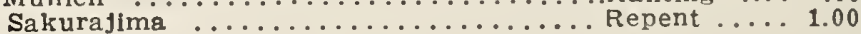

100 lbs.

$\$ 30.00$

25.00

35.00

30.00

30.00

35.00

30.00

35.00

25.00

28.00

35.00

25.00

30.00

25.00

35.00

50.00

30.00

30.00

30.00

25.00

25.00

30.00

30.00

40.00

35.00

30.00

28.00

35.00

30.00

40.00

25.00

30.00

35.00

35.00

35.00

35.00

35.00

30.00

30.00

35.00

35.00 
S.ILSIFY.

Tel. Cipher. Lb. 100 lbs.

Mammoth Sandwich Island

Sacker ...\$0.55

$\$ 50.00$

SCORZONERA, or Black Salsify...........Sacristan . . 1.00

SORliEI, Broad-I.eaved French............Sagacity . . . 1.25

SIINACH. Write for prices on larger lots.

I3loomstale, or Norfolk, Savoy Leaved.... Salem .... . .12

Extra Iarge Round-Lcaved..............Saltern ..... 12

Large Round Viroflay............................... 12

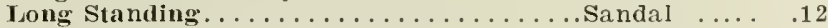

Vaughan's Giant Fill Basket (new)...............15

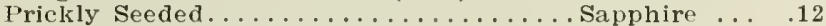

Tetragonia, or New Zealand............Sanguine ... .35

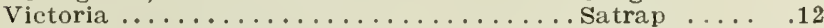

Vaughan's Triumph............... Satrox .....14

Swiss Chard............................ .50

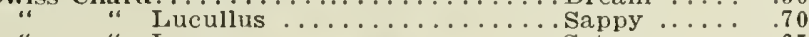

Lyon ...............................

10.00

10.00

10.00

10.00

13.00

10.00

30.00

10.00

12.00

45.00

65.00

60.00

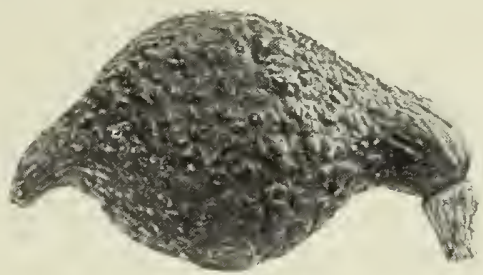

CHICAGO WARTED HUBBARD.

\section{SQUASH.}

Boston Mariow ................ Saver ....\$0.40

Chicago Warted Hubbard ................. Scaffold .... .55

Chicago Orange Marrow ............... Scabbard ... .45

Delieious ............................55

Delicata ............................... 50

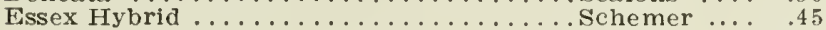

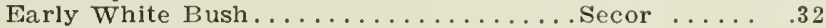

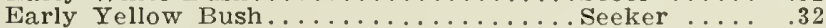

Faxon's Brazilian ......................... .45

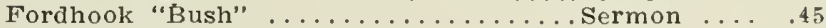

Fordhook Early .......................... 50

Giant Summer Crookneck ...................... .45

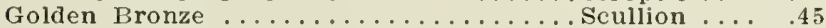

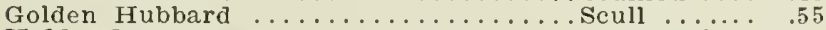

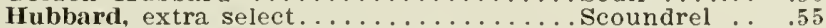

Mammoth White Bush, extra select... Scutcheon .. .35

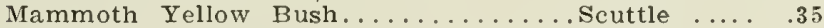

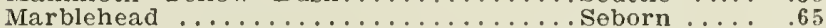

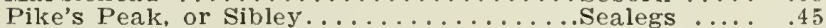

Vegetable Marrow ................ Sectary .... .50

White Summer Crookneck.............Sedan ...... 35

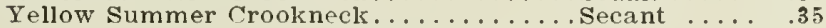

\section{TOBACCO.}

Big Havana . . . . . . . . . . . . Tabard . . \$2.25

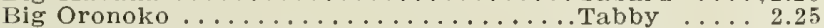

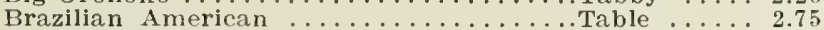

Comstock's Spanish .............. Talmud ... 3.00

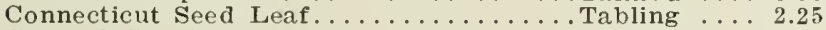

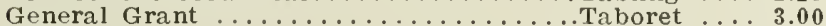

Harby .................... Tape ..... 2.75

Primus ........................... 2.25 


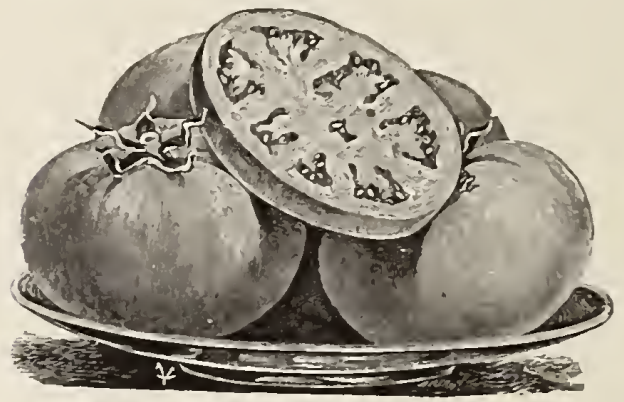

NEW STONE.

\section{TOMATO.}

All our seed is contract grown by the most reliable and experienced men. It can absolutely be relied upon.

Vaughan's Earliest of All.

Tel. clpher.

Lb. 10 lbs.

Vaugluan's Model .................... Thaning ... 1.75

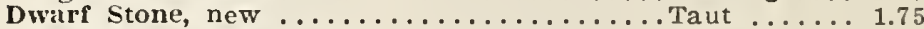

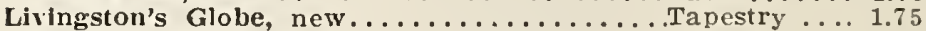

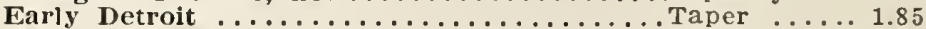

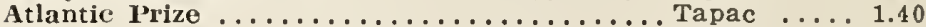

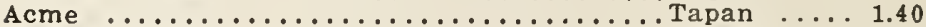

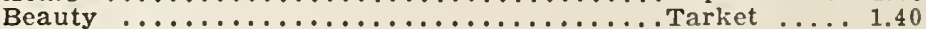

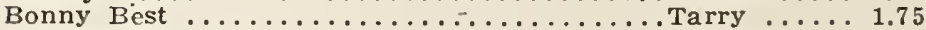

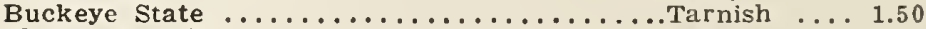

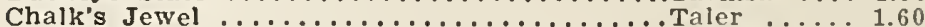

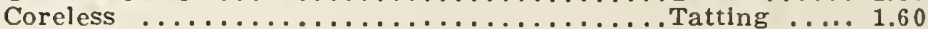

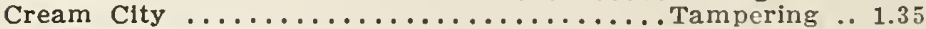

Crlmson Cushion ............................. 2.25

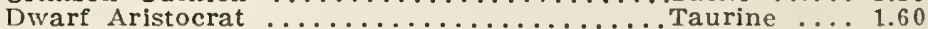

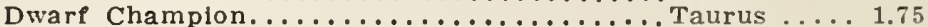

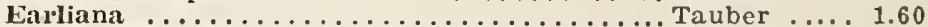

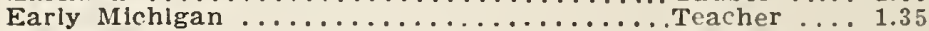

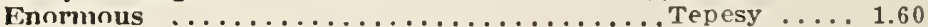

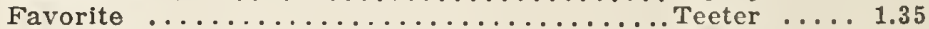

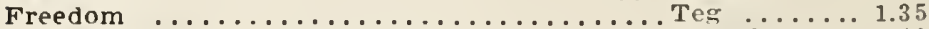

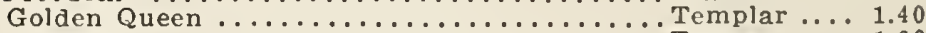

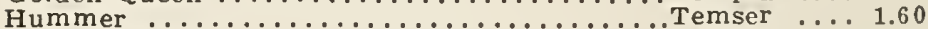

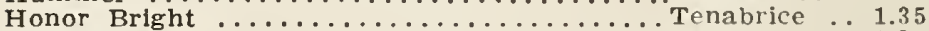

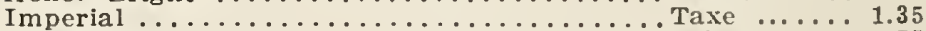

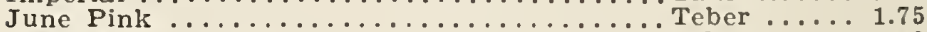

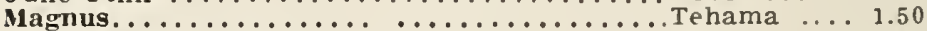

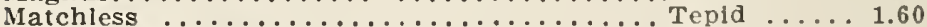

Pear-shaped Yellow................. Tapa ..... 1.50

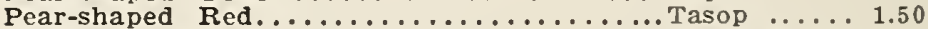

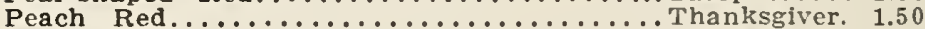

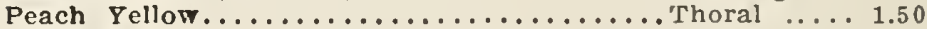

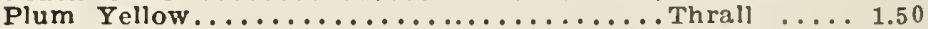

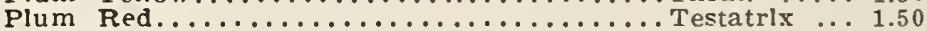

Perfectinn ....................... Testanlng ... 1.35

Ponderosa ............................. 3.00

Stone, Impreved. .............................. 1.40

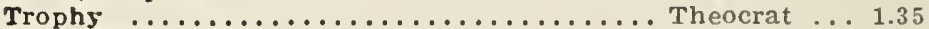

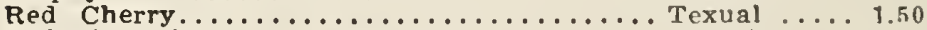

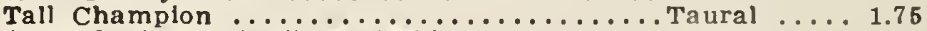

Ground Cherry (Yellow Husk).............. Temptation . 1.50

$\$ 14.00$

16.50

16.50

17.50

12.50

12.50

12.50

16.50

14.00

15.00

15.00

12.50

21.50

15.00

16.50

15.00

13.00

15.00

12.50

12.50

13.00

15.00

12.50

12.50

16.50

$14 .(11)$

15.00 


\section{Tonnato Forcing Sorts.}

Frogmore, selected ................. Teheram ...\$2.u0

Hubert's Marvel ....................... . . . . . . . . 2.00

Carter's Sunrise ................................ 2.00

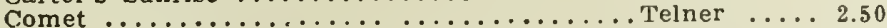

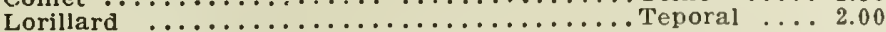

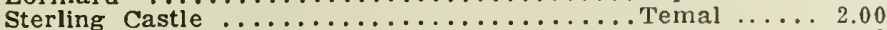

Sutton's Best forcing................ Theo ..... 2.00

TURNIP.

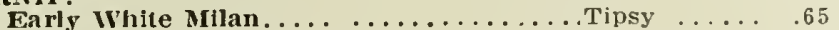

Extra Early Purple Top Milan......... Tibing ..... . . 65

Purple Top Strap Leaf, choice......... Thugord ... . .25

Purple Top White Globe.............. Tirode ..... .28

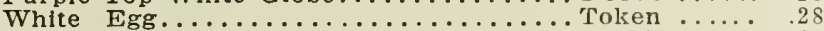

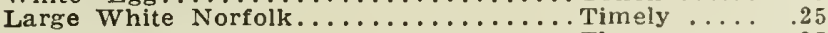

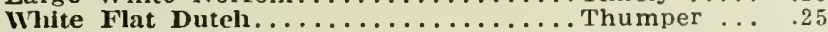

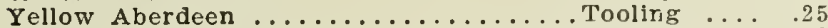

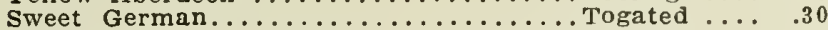

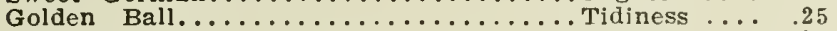

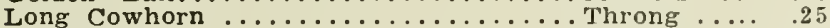

Amber Globe .................... 25

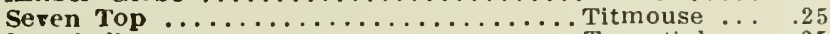

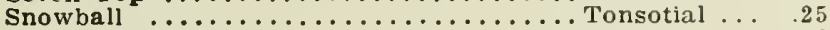

Rhode Island Rock........................ 40

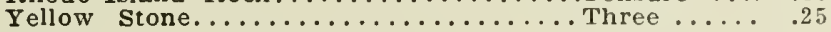

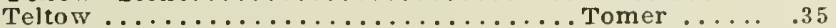

$10 \mathrm{lbs}$.

$\$ 19.00$

19.00

$100 \mathrm{lbs}$

60.00

60.00

22.00

25.00

25.00

2200

22.00

22.00

28.00

22.00

22.00

22.00

22.00

22.00

22.00

30.00

SIVEDES OR RUTABAGAS.

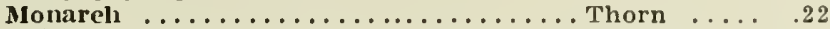

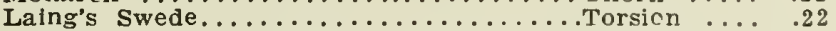

20.00

Carter's Improved Swede...........Topmed ... .22 20.00

Vaughan's Imp. American, Purple top.... Topic ...... .25 23.00

Skirving's .................... Toward .... .24 22.00

Sutton's Champion, extra fine..........Tracking ... $.25 \quad 22.00$

Breadstone $\ldots \ldots \ldots \ldots \ldots \ldots \ldots \ldots \ldots$ Thriff $\ldots \ldots \ldots .25 \quad 22.00$

White Swede..................Tramper ... $.22 \quad 20.00$

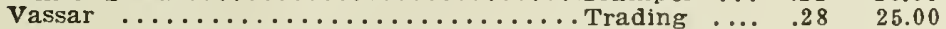

\section{FAR $M$ and MISCELLANEOUS}

Prices Change; write for latest quotations. Bags Extra. Terms Cash.

Items with * F. O. B. New York or Chicago. Balance of list F. O. B. Chicago. Ask our New York House for Quotations. Items with no prices attached are subject to quick market changes. Latest quotations on application.

Barley, Success 48 lbs. to the bushel....... Wolf ....

Manshuri, $48 \mathrm{lbs}$. to the bushel......W Wollab

"Wisconsin Pedigree ............... Wollan

Barley, White Hulless, Montana grown........ Woland

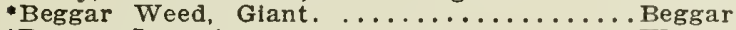

* Bromus Inermís ..................... Warouse

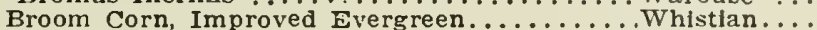

Buckwheat Japanese, write for prices............. Bucking

Buckwheat Silver Hull, write for prices....... Buckram

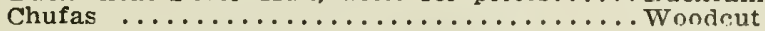

Corn, Kaffir, White.................Wobweb

$.13 \quad 11.00$

CORN.-Field (Write for latest prices.) -

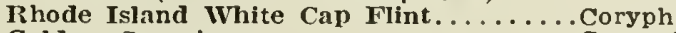

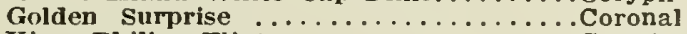

King Philip. Flint......................

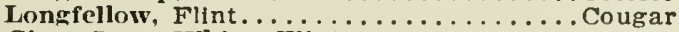

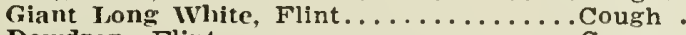

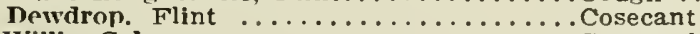

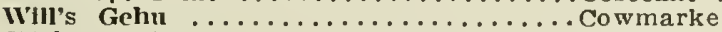

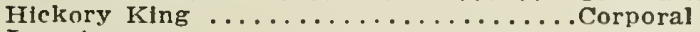

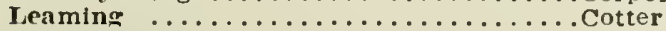

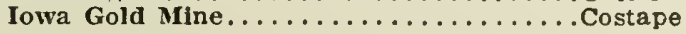


CORN, Field-Continued.

Iowa Silver Mine.......................

Tel. clph. Lb. $100 \mathrm{lbs}$.

Minnesota No. $13 \ldots \ldots \ldots \ldots \ldots \ldots \ldots$ Corner

Pride of the North.

Cousin

Reld's Yellow Dent on the Ear......... Cowmuling

Reid's Yellow Dent shelled............ Cowhide

Silver King (Wisconsin No. 7 )............... Coulter

Boone County White Dent............... Coward

Madison Yeliow Dent, Best early............ Cort

Sunshine Dent, Earliest................ Corner

CORN-Pop, all selected ears for secd.

Mapledale Prolific................. Crater

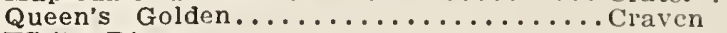

$\begin{array}{rr}\$ 0.05 & \$ 4.00 \\ .05 & 4.00 \\ .05 & 4.00 \\ .05 & 4.00 \\ .08 & 6.00\end{array}$

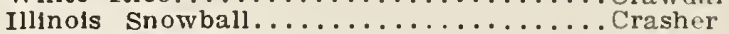

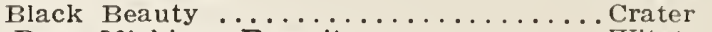

Cow Peas, Michigan Favorite.............. Witator

Cow Peas, Black-Eye................Witation

Cow Peas, Whlppoorwill ...............Witric

Cow Peas, Mixed.

Witrock

Espersette, or Sainfoin.

Witwell

At market price

At market price

At market price

Flax Seed, write for prices................... Witword $\ldots$

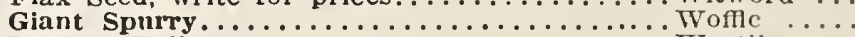

Lupins, Yellow..................................

Lb. $100 \mathrm{lbs}$

Maw, blue.

Whigol

Whimney

Milo Malze, White.

Wood ......

Millet, Pearl or Cat Tail

Womate

Oats, Black

Wome

Lincoln

Womcdy

Victory

Wometer

Peas, Canada Field, write for prices........... Parasite

- Peanuts, Spanish ...................... Pandote

- Peanuts, Mammoth Virginla.................. Pandave

- IRape, Essex......................... Wodif

Salt Bush, Australian................... Woodecock

Sand Vetch $\ldots \ldots \ldots \ldots \ldots \ldots \ldots \ldots \ldots$ Woffer

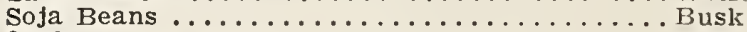

Speltz $\ldots \ldots \ldots \ldots \ldots \ldots \ldots \ldots \ldots \ldots$. Speltz

Sugar Cane, Early Amber.............. Whisk

- Sunflower, Mammoth Russian............... Whough

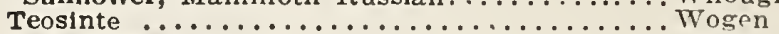

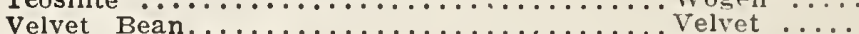

so.14

"VETCHES, Spring ................. Wognate

-WHEAT, Klondyke ................... Woolo .....

.08

.08

.60

$.14 \$ 13.00$

Woolman

Wollyt .....

Woolsack ...

Woolweb . Write for prices

Woolwoven..

Wompart ...

Whapter

\section{TREE SEEDS}

WINTER R
WILD RICE.

Tel. cipher.

Ash, White

Tondem

Box Elder....................... Wonduct

Catalpa Speciosa. True

Wonfall

Wonfront

Locust, Honey

Wonform

Won Wins

Lb. $100 \mathrm{lbs}$.

$\$ 0.30 \$ 25.00$

1.25

20.00

.40

35.00

Maple, Oregon Mammoth.

.50

1.10

Osage Orange.

ALL GRASS AND CLOVER SEEDS.-Alfalfa, Alsyke, white Clover.

Crimson, Medium Red, and Mammoth Clorer, etc. Prices on application. 


\section{VAUGHAN'S \\ Trade List of Flower Seeds.}

\section{"THE BEST FLOWER SEEDS IN AMERICA."}

Our list contains all the best novelties we know to be worthy of cultivation snd are improvements on the older kinds or entilely new valieties, as well as all the standard sorts. The quality of our seeds is the very best, and our prices are reasonable.

A Quantities of $1 / 4$ lb. and over are charged at the pound lates (1/8 $1 \mathrm{~b}$. at the oz. rate), $1 / 2$ oz. at the oz. rate, but only when the price per ounce is $60 \mathrm{c}$, $\mathrm{or}^{\circ}$ more, and is not otherwise quoted.

We do not sell half ounces where the price per ounce is $15 \mathrm{c}$ or under.

Abobra Viridiflora, Ornamental Cucumber....... Abapo . . $\$ 1.00$

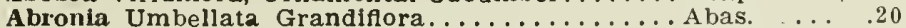

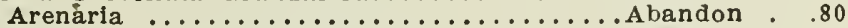

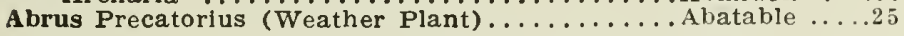

Abutilon fine mixed..........per 1/8 oz., 30c..Abavus ... 1.80

New Hybrids, mixed........ per $1 / 8$ oz., 50c..Abba ... 3.00

Acacla Lophanta Speciosa Compacta........... Abdera ... . 10

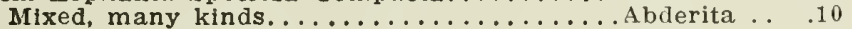

Acanthus Latifolius, very decorative. . . . . . . . . . Abdite ... . . . . . . . . . . . . .

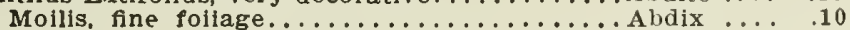

Aclillea The Pearl, very fine.....1/8 oz., $40 \mathrm{c}$. Abditery .. 2.60

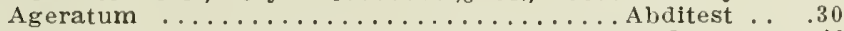

Aconitum Napellus, Monk's Hood, blue......... Abdo .... .40

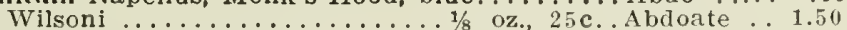

Aoroclinium Roseum, pink..................................... .10

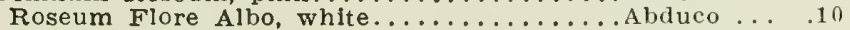

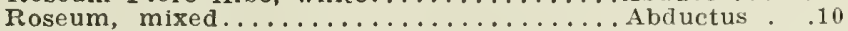

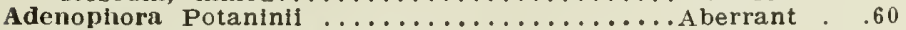

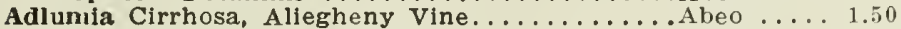

Adonis Aestivalis, (Flos Adonis), red............ Aberro . . . . 10

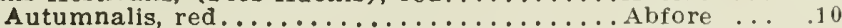

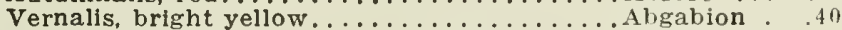

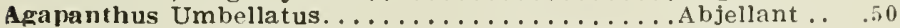

Agathaca Coclestis (Amelloides), Blue Daisy...... Abhor ... .70

Agerafum Mexicamim, biue....................... 10

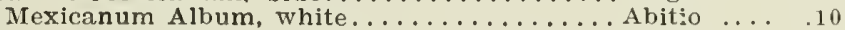

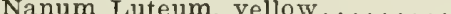

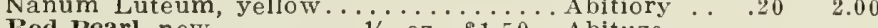

Imperial Dwarf Biue................... . . . . . . . . $25 \quad 2.80$

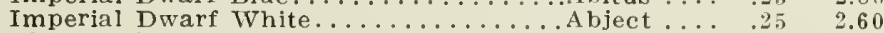

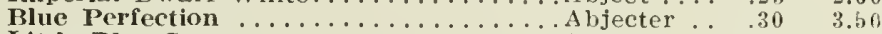

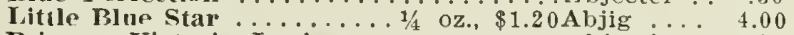

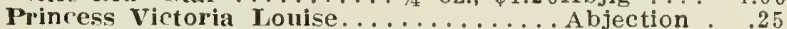

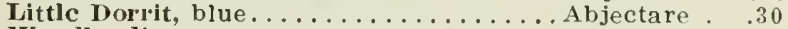

Wendlandi nanum conpactum album..... Abjectori $\quad .30$

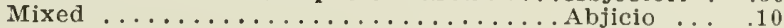

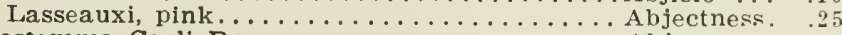

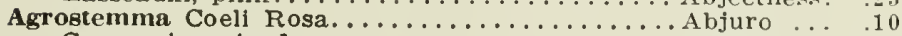

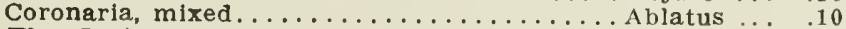

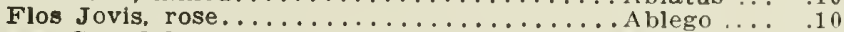

2.50

3.00

1.20

2.80

.70

1.00

1.00

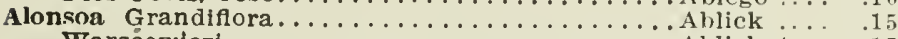

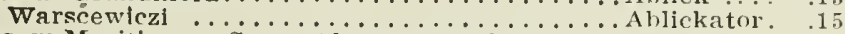

Alyssum Maritimum, Sweet Alyssum 10 ibs., $\$ 10.00$ Ablutto . . . 10

Bentham! Compactum Erectum, dwarf......Ablutus ... . .20

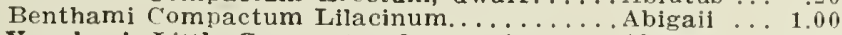

Vaughan's Little Gem, extra fine strain . . . . . . Abnego ... .40

Saxatile Compactum, Gold Dust..................... . 25

1. 60

1.20

2.00

4.00

2.50 
TEL. CIPHER.

Amaranthus Tricolor, Joseph's Coat........... Abnuo ...\$0.15

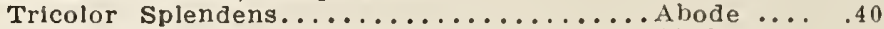

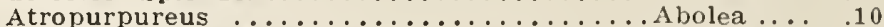

Caudatus (Love Lles Bleeding), red.........Abolitus . . .10

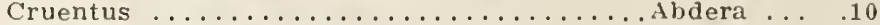

Superbus (Coleus-leaved)...................... .10

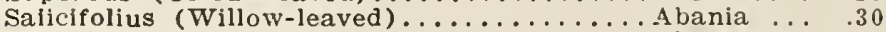

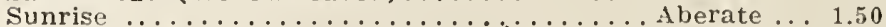

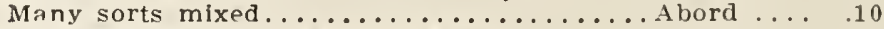

Amaryllis, new hybrids, $75 \mathrm{c}$ per 100 seeds.......... Abording

Amberboa Muricata Rosea.................. Aborford

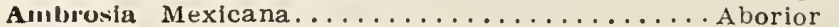

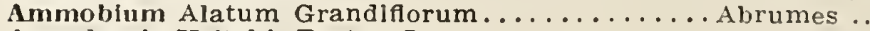

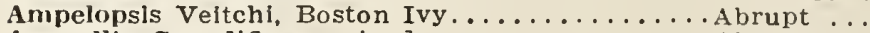

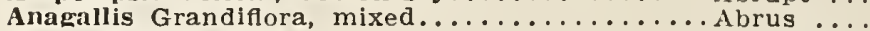

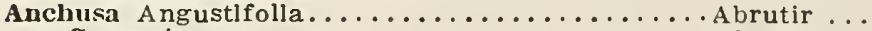

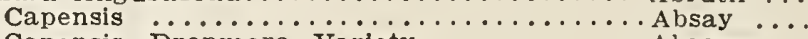

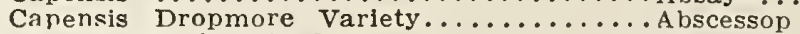

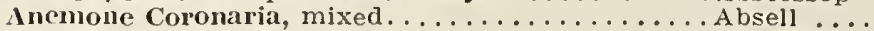

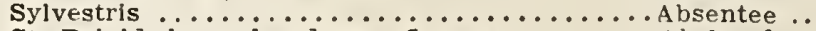

St. Brigld, large handsome flowers........... bshead.

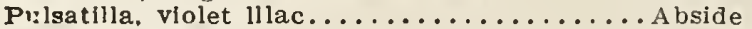

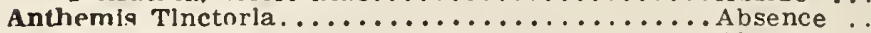

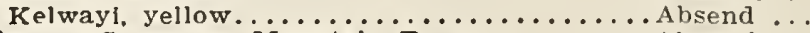

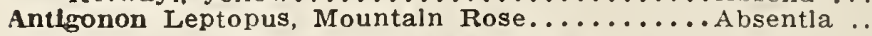

\section{ANTIRRIINUM MAJUS GRANDIFLORUM.}

Delila, carmine and yellow, white throat........ bsclay ... .30

Firefly, scarlet, white throat.........................30

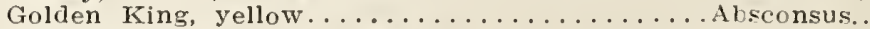

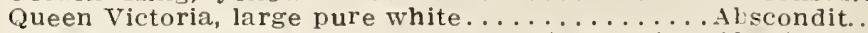

Silver Pink...............1/8 oz., 60 c. Aborforge.

Striped Varieties, mixed (Butterfly).............

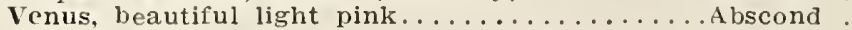

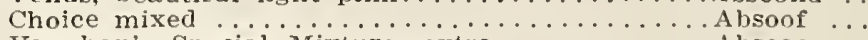

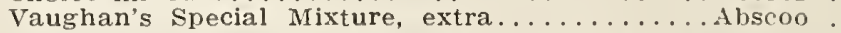

\section{ANTIRRIIXCM M.J'S.}

Atrococcincum, dark scarlet...........................

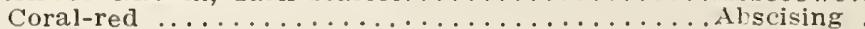

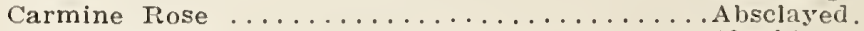

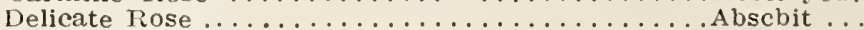

Fairy Queen, golden orange with white throat...Abscbited.. Maximum Purple King................. Abscbiting.

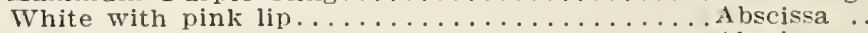

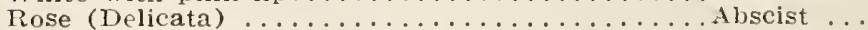

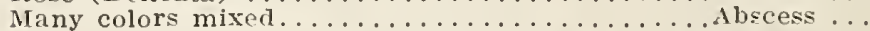

\section{INTIIRIINUM MAJT NANIM.}

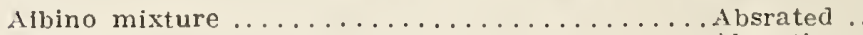

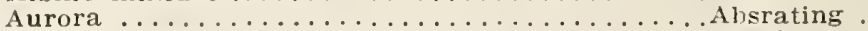

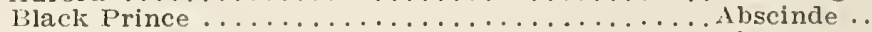

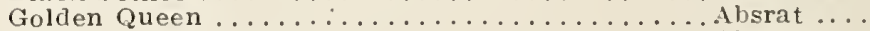

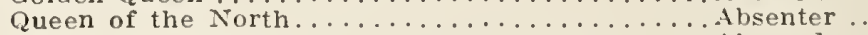

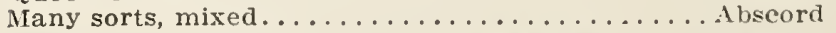

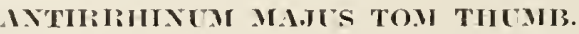

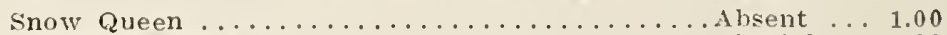

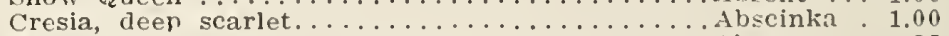

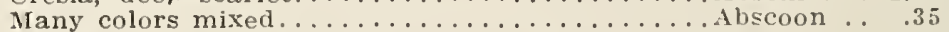

Aquilegia Columbine, Vulgaris, single mixed ...... Absillio ... . .20

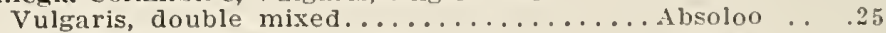

Canadensis, scarlet and yellow.......................... 1.00

Chrysantha, yellow ................................................... 1.20

Chrysantha Grandiflora Alba, white.......... A sone ... 1.25
LB.

$\$ 1.40$

.40

.35

.40

.70

3.00

20

2.00

.80

1.35

2.00

2.00

3.00

2.50

3.50

3.50

3.20

3.50

3.50

6.00

2.30

4.50

3.50

3.00

3.50

4.00

3.00

6.00

4.170

4.00

2.00

๖. 00

3.50

3.50

3.50

3.20

2.00

4.00

2.20

2.60

16.00 16.00 


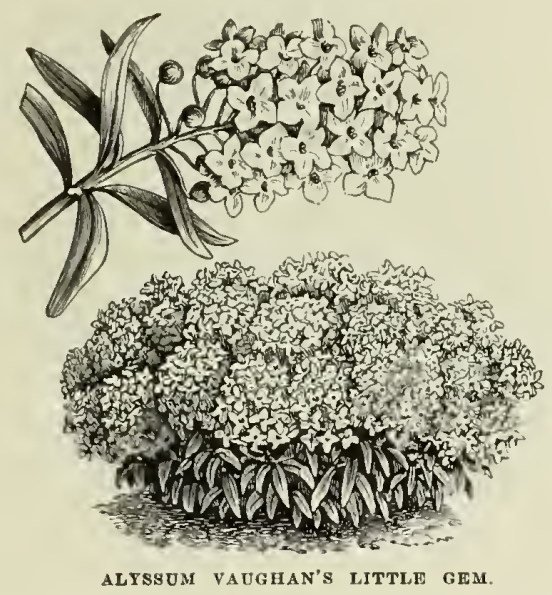

AQUIHEGIA-Continued.

TEL. CIPHER. OZ.

LB.

Chrysantha Fl., pl., double................ Absorb ..\$1.40

Helenae, very fine .............................. 5.00

Improved Long Spurred English Varieties... . Absurde .. .85

Vulgaris Alba Plena, double white........... Absorbeo . .40

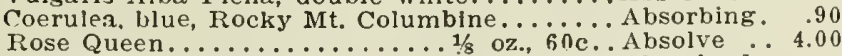

$\$ 12.00$

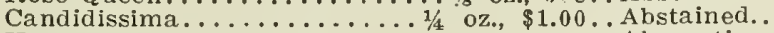

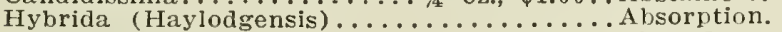

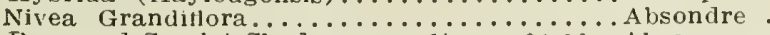

1.2516 .00

Rose and Scarlet Shades...... $1 / 8$ oz., $\$ 1.00 \ldots$ Absorp ...

Skinueri, scarlet tipped with green........... Absorpate. 3.00

Vervaeneana Atroviolacea Plenissima........... Absumere . .60

Vaughan's Special Mixture............................ 1.00

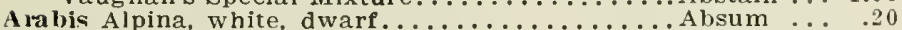

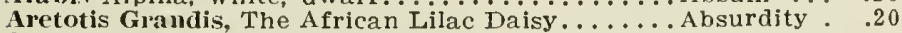

2.40

Arenaria Cacspitosa ........... Montana ...............

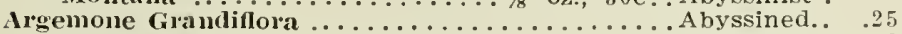

Aristolochia sipho, Dutchman's Pipe.............................. 50

12.00

$25 \quad 2.50$

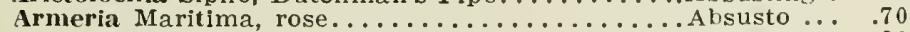

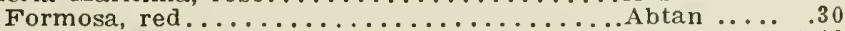

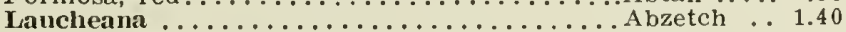

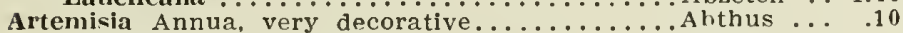

2.20

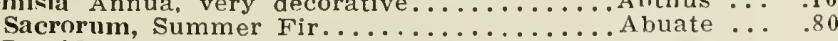

6.00

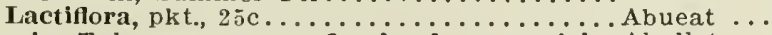

Aselepias Tuberosa, orange, fine hardy perenntal . . Abullot ... 1.00

\section{ASPARAGUS.}

Asparagus Plumosus Nanus, Northern Greenhouse Grown 1,000 seeds at $\$ 3.50$ per thousand; 5,000 to 10,000 seeds at $\$ 3.25$ per thousand; 11,000 to 25,000 seeds at $\$ 3.00$ per thousand; 26,000 to 50,000 seeds

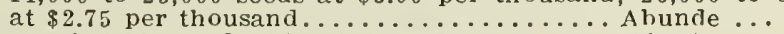

Sprengeri, 1,000 seeds, $50 \mathrm{c} ; 100$ seeds, $15 \mathrm{c} \ldots \ldots$. . A busive $\ldots . .30 \quad .300$

Asparagus Hatcherii ........\$5.00 per $1,000 \ldots$ Aburria ...

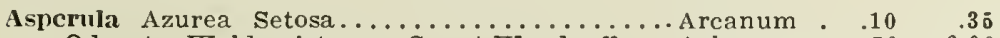

ninrata. Waldmeister, $\mathrm{nr}$ Sweet Woodruff.....Acbarus .. $\quad .50 \quad 6.00$

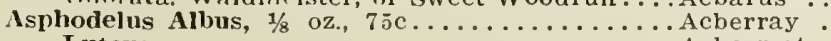

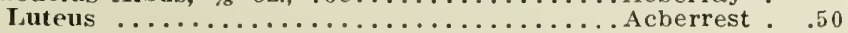




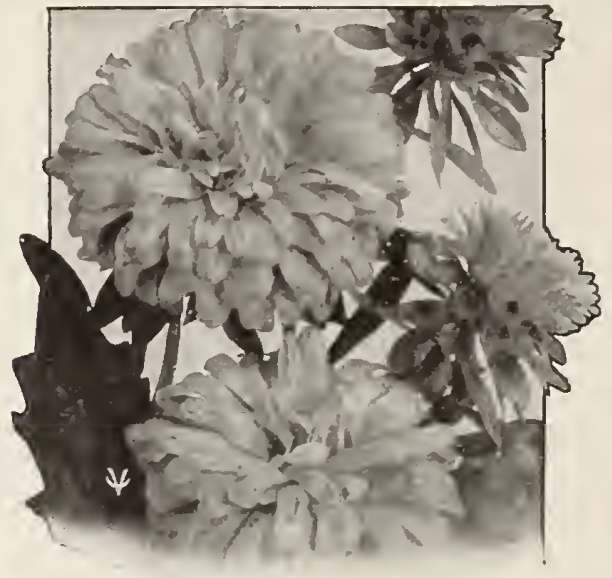

ASTER QUEEN OF THE MARKET

\section{ASTERS.}

Our Aster seed is raised by experienced and relíable seed growers, in some cases from our own stock seed and should not be compared with btock ralsed in the south or by experimenters who use no care and lack experlence.

Vaughan's Litte Gem, helght 6 Inches, flowers

$$
\text { TEL. CIPHER. OZ. L. }
$$

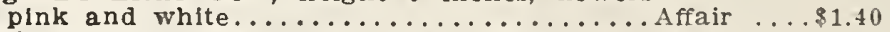

Vaughan's Fireball, a splendid dwarf Aster...... Agast .... 1.60

Vnughan's Snowball, the best dwarf white Aster.. Agastria .. 1.50

\section{Dwarf Chrysanthemum Flowered Aster}

TEL. CIPHER.

White

White, turning to rose... Afloat.

Peachblossom .........Agalman.

Brilliant Rose.......... Afric.

Crimson .............. Afront.

Fiery Scarlet. . oz., $\$ 1.60$. Aftaba.
TEL. CIPHER

White, turning to azureblue............... Afoot. Light Blue and White. Again. Light Blue.............. galma

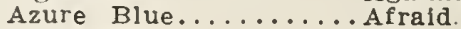
Dark Blue................ Afresh

Each of above, except noted, per 1/4 oz., $35 \mathrm{c}$; per oz., $\$ 1.30$. TEL. CIPHER. OZ. LB.

Dwarf Chryanthemum-Flowered, all colors mlxed. Agaric . .\$0.\$5 $\$ 10.00$

\section{Dwarf Queen Aster}

TEL. CIPHER

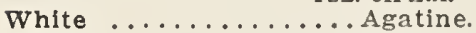

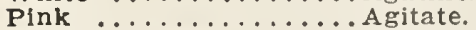

Rrilliant Rose.........Agitator.

Flesh............... Ageta.

TEL. CIPBER

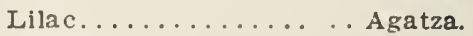

Crimson ............... Age.

Dark Blue........... Agitable

Each of the above, per oz., \$1.20.

Tel. Cipher. Oz. Lb.

Dwarf Queen, all colors mixed..................

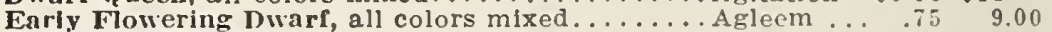

Gloria or Button-hole. The flowers are about 2 Inches in diameter, pure white with a scarlet margin ............................. 2.00 
TEL. CIPHER.

Oz.

LB

Pigny, Dwarf Bouquet or Boltze's, choice mixed . Aflight . . \$0.75\$9.00

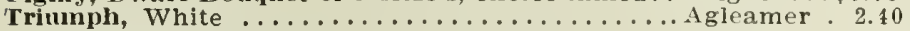

Scarlet ........................... 2.40

Mixed, many colors ................. Aglee ... 1.50

16.00

\section{COMET ASTER.}

TEL. CIPHER.

TEL. CIPHER.

White .............Adjourn.

Brldesmaid, white turn-

ing to lilac rose....... Adleu.

Peach Blossom......... Adjunct.

Pink, bordered white... Adjudge.

Dark Rose............. Adjournal. Each of above.

Carmine ............ Adjusted.

Deep Searlet.......... Adjust.

White and Lilac........ Adjuster.

Light Blue and White...Adjure.

Light Blue............. Adjugate.

Dark Blue............. Adjustant.

per oz., $\$ 1.00$.

Comet, all colors mixed

TEL. CIPHER.

Oz. LB.

... Adjustria .\$0.65 \$8.00

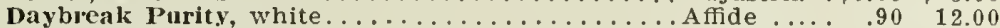

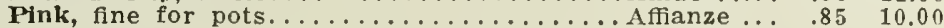

Salmon Pink

1.50

\section{GIANT COMET ASTER.}

Thite CIPHER,

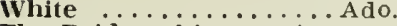

The Bride, white turning

to $p \operatorname{lnk} . . . . . . . .$. Adopt.

Queen of Spain, soft yel-

low turning to flesh.. Adopting.

Sulphur Yellow.........Adog.

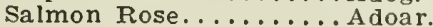

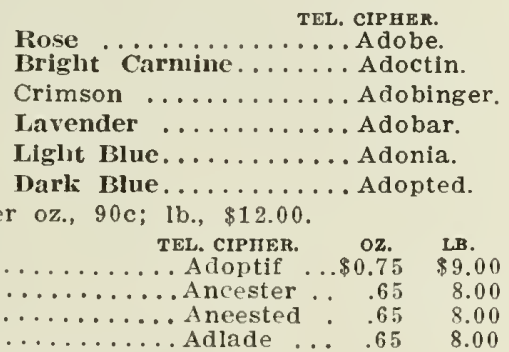

Giant Comet Aster, all colors mixed.............. Tdoptif $\ldots . \$ 0.75 \quad \$ 9.00$

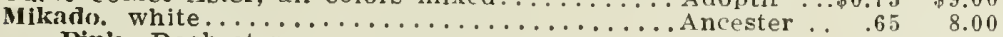

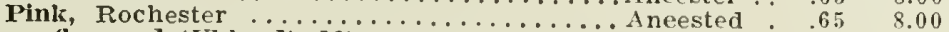

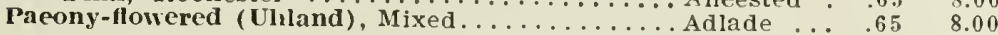

\section{QUEEN OF THE MARKET ASTER.}

TEL. CIPHER.

White .........................

Pink ................ Adversely.

Crimson.............. Advisable.

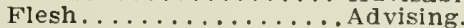

Azure Blue ............ Advisage.
TEL. CIPBER.

Dark Lilac............ Advist.

Silvery Lilac.......... Advistare.

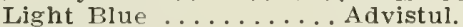

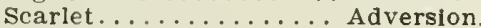

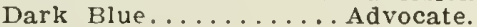

Fach of the ahove, per oz., $50 \mathrm{c}$; per 1b., $\$ 6.00$.

TEL. CIPHER. OZ. LB.

Adrock $\$ 0.40 \quad \$ 4.50$

Queen of the Market, all the above, best mixed . . Advock ...\$0.40 \$4.50

\section{VICTORIA ASTER, Vaughan's Improved.} Acutule.

MIss Roosevelt, yellow

turning to flesh.... Admittance. Apple Blossom......... Adaquo.

Brlght Pink.................. Adhíbeo.

Crimson and White.......Adight.

Dark Crimson........... Adaucto.

TEL. CIPHER

Bright Scarlet. . . . . . . Additio.

Light Blue............ Adaxint.

Azure Blue............ Adamus.

Indigo Blue on white ground ............. Adlnole.

Dark Blue and White... Adesus.

Dark Blue........... Adelphia.

Many Colors, in splendid mixture............ Adipous . $\$ 1.00 \$ 14.00$ Victorla Aster, imp. col., 6 col., each, net, 20 c... Aditus..... imp. col., 12 col., each, net, $40 \mathrm{c}$... Adition.... Each of the above, per oz., $\$ 1.60$. 


\section{BRANCHING OR SEMPLE ASTER.}

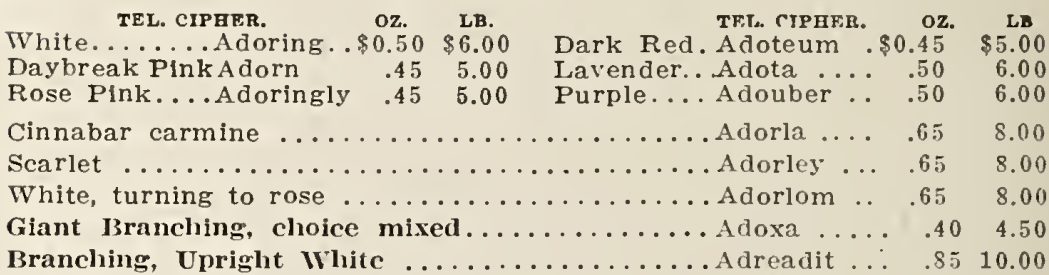

\section{CARLSON OR INVINCIBLE ASTER.}

White

TEL. CIPHER.

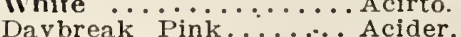

Marquis Pink.......... Actavus.
TEL. CIPHER.

Lavender ............Acme.

Violet Blue...........Acnop.

Each of above, oz., $\$ 1.00$.

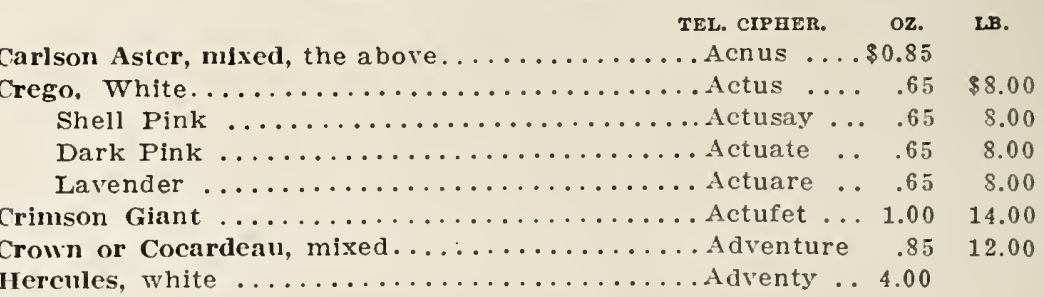

\section{HOHENZOLLERN ASTER.}

TEL. CIPHER.

Bride, white turning to

pink ............... Adosomer.

Pink ............... Adoreting.
TEL. CIPHER.

.......Adorx

Crimson ...............Adourner.

Silvery Lilac..........Adorus.

Light Blue............. Adorvas.

Each of the above, oz., $\$ 1.40$.

TEL. CIPHER. OZ. LB.

Hohenzollern, many colors, mixed............ Adoryus ...\$0.90 $\$ 12.00$

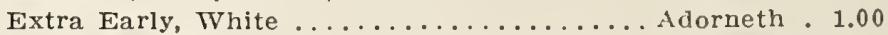

Extra Early, Rose ............................ 1.00

\section{VAIIOCS ASTEIR.}

Ostrich Feather, many colors, mixed..........Adorare .. .85 10.00

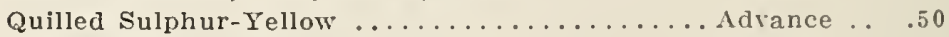

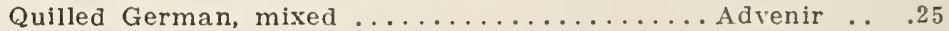

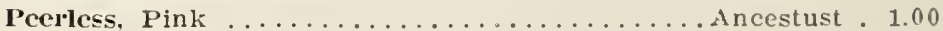

Pink Bcauty ....................... Adormate . 2.00

Enchantress Pink ................... Adormey . 1.80

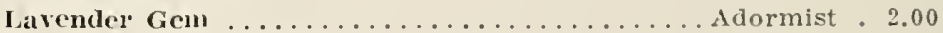

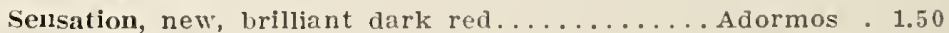

Unicum White.................................. 2.00 


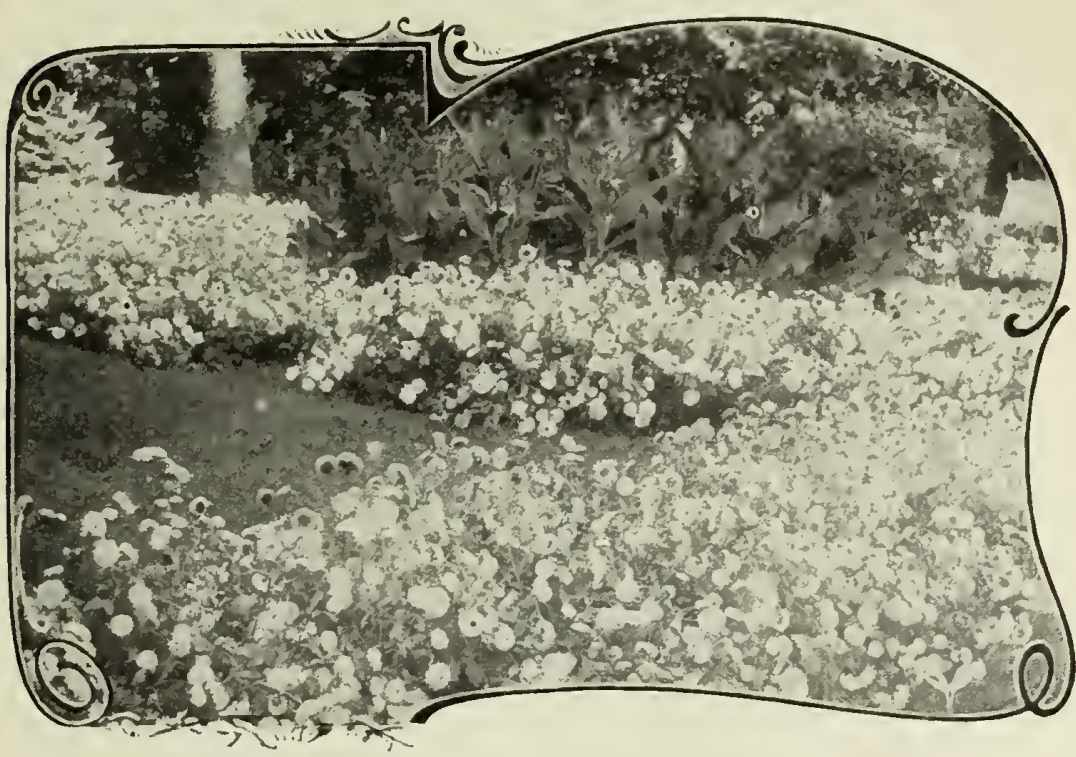

BED OF VAUGHAN'S GIANT' BRANCHING ASTERS

\section{ASTER}

\section{PAEONY-FLOWERED PERFECTION.}

TEL. CIPHER

TEL. CIPHER

Snow White ........... Accensus. Sinlight, sulphur-yellow. Acinose. Delicate Rose ........ Accera.

Surprise, chamois

turning to pink......Acinetan. Pink (La Superbe).... Accepso. Crimson .............. Accido.
Brilliant Scarlet........Aceo.

Gravelotte, Bloodred.... Accurbus. Light Blue and White... Acesco.

Ametliyst Blue.......... Achiote.

Light Blue............ Acerbus.

Dark Blue.............. Accolo.

Shining Blıc-black..... Acerbe.

Each of the above, per oz., \$1.25; 1/4 oz., $35 \mathrm{c}$.

Paeony-Flowcred Perfection, splendid mixture... Acena $\ldots \ldots 0.75 \quad \$ 9.00$

TEL. CIPHER. OZ. LB.

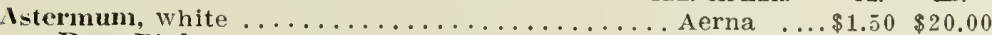

Rose Pink . . . . . . . . . . . . . . . . . Aernope .. 1.5020 .00

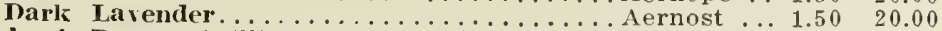

Vaughan's Beauty, brililant red.................. Afferent .. $\$ 1.25$

\section{IIING ASTERS.}

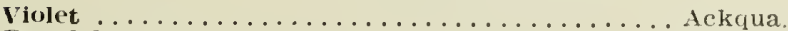

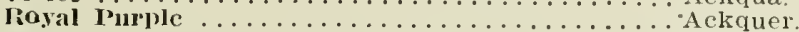

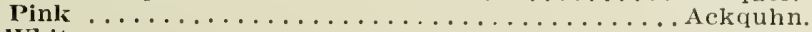

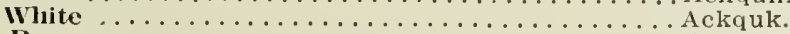

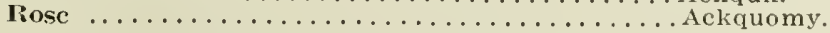

Each of the above. $1 / 4$ oz, $25 \mathrm{c}$; per oz., $85 \mathrm{c}$; per $1 \mathrm{~b} ., \$ 12.00$.

Elcetrotypes of our catalog illustrations can be furnished at $15 \mathrm{c} p$ er sq. in. 


\section{ASTERS-Continued}

Vaughan's Florists' Mixture. . . . . . . . . . . Agrippa . \$1.00\$14.00

Wlite Tall Varieties, mixed....................... .75 10.00

Dwarf Asters, many classes and colors mixed.... Agreet ... $\quad .50 \quad 6.50$

Tall Asters, many classes and colors mlxed.......Agreement $\quad .30 \quad 3.50$

Vaughan's Exeelslor Mixture of Dwarf Asters. It is comprised of the most distinct and desirable colors of the different classes of Dwarf Asters speclally selected for this mlxture. Agricole . 1.2516 .00

Vaughan's Exeelsior Mixture of Tall Asters. Agrilus.

This ls a mixture of the most beautiful, striking and distinct colors of the tall growing Asters, selected from the cream of the Peony Fld. Vietoria, Glant Comet, Hohenzollern, Ostrieh Plume, Carlson, Branching and other desirable sorts, and includes many klnds specially purchased for this mixture. Per oz., $\$ 1.25 ; 1 \mathrm{~b} ., \$ 16.00$.

TEL, CIPHER.

$0 \%$.

LB.

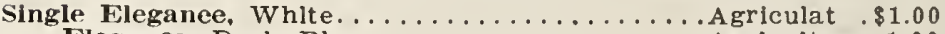

Elegance, Dark Blue................... Agrlcultna. 1.00

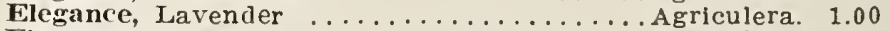

Eleganee, Rose ............................ 1.00

Ierennial Asters, choice mixed. $1 / 4$ oz., $20 \mathrm{c} \ldots \ldots$ Ahoy .... .65

Aubretia Hyb. Grandifiora. 1/\& oz., $\$ 1.00 \ldots \ldots \ldots$ Average

New Large Flowering Varieties Mixed............ A hoyage .. 1.00

Aurlcula (Primula), fine mixed, $1 / 8$ oz., $65 \mathrm{c} \ldots \ldots$.... Aulpha ... 4.00

Bachelor's Button, mixed (see also Centaurea)... Cede...... .10

Balloon VIne (Cardiospermum Halicacabum).... Babakoto . .10

\section{BALSA MS-DOUBLE.}

TEL. CIPHET.

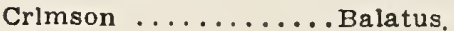

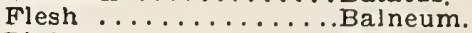

Pink ................. Balteus.

Scarlet $\ldots \ldots \ldots$. Bamballo.

White............. Bare.
Crimson Spotted ......Barbaos.

Solferlno ........... Barbula.

Lllac ................ Barcae.

Light Lemon..........Barcel.

Dark Blue ..........Barcine Each of the above, oz., $30 \mathrm{c}$ : 1b., $\$ 3.00$.

TEL. CIPHER, OZ. LB.

Atrosanguinea plenissima, double blood red..... Bacenite . $\$ 0.30 \$ 3.00$

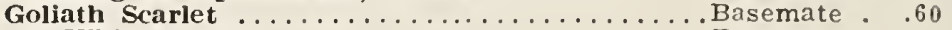

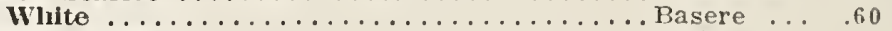

Malniaison Pink................................. 40

Prlnce Bismarek, salmon rose....................40

Vaughan's Invinelble, the best mixture in existence.Baris .... $\quad .50$

Alba Perfecta, or white Deflance, best double white.Barisine . . .40

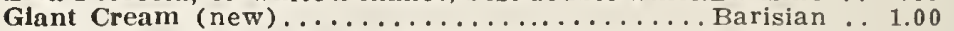

Double Cannella-Flowered, all colors best mixed..Barium ... 25

Inproved Double Camella-Flowered, cholce mxd.Barnacle . . .50

Double Dwarf, mixed..................... Baro ..... .20

Double Rose-Flowered. best quality mixed . . . . . Basilia . . . .25

Donble Carnation Striped, mixed............. Basilicus . .20

Tall Double, mixed colors.......5 Ibs., \$7.50 . Bassaris .. .15

Balsam Apple, (Momordica Balsamina) ........ Beatus ... .15 1.so Pear, (Momordica Charantia) ....................... $20 \quad 2.00$ Apple and Pear, mlxed........................... . 1.80

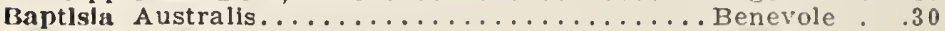

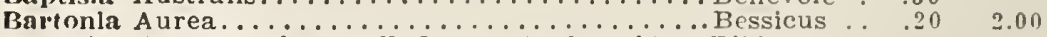

Begonla, Single Tuberous Rtd.. mx., 1-32 oz.\$1.00 Riblus.... Dbl. Tuberous Rtd., $m x$. pkt. (500 seeds) $17 \mathrm{c}$ net. Bibo ...... 


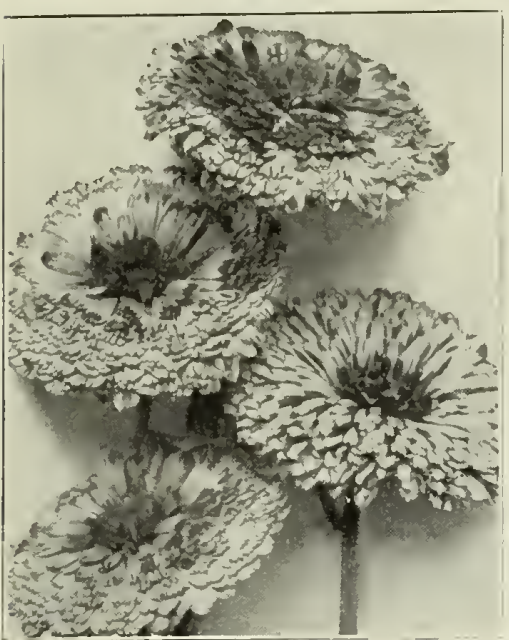

CALENDULA

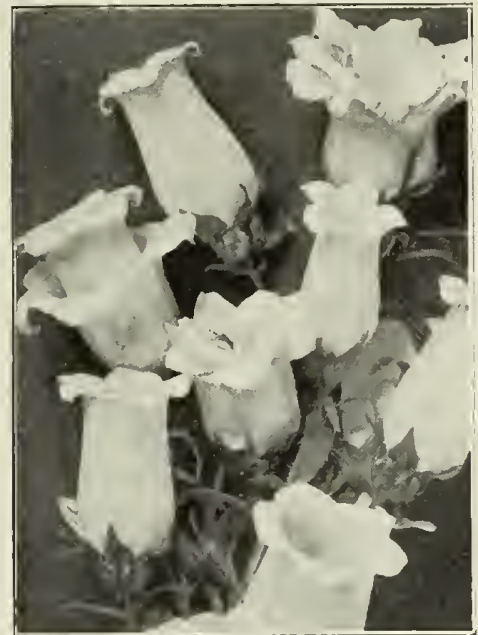

CAMPANULA-Canterbury Bells

Begonia.

TEL. CIPHER.

oz.

LB.

EIfordia, rosy carmine....1-16 oz., \$1.00.. Bibolation

Erfordia Grandiflora Superba.1-32 oz., \$1.50 . . Bibozat . .

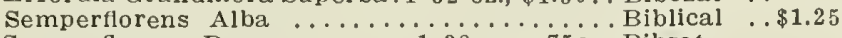

Semperflorens Berna........1-32 oz., 75c..Bibsat ....

Semperfforens Mixed ..................... .75

Gracilis Luminosa ........ 1-32 oz., $\$ 1.00 \ldots$ Bibolate ..

Gracilis Bonfire............pkt., 12c net.. Bibozene .

Gracilis Mignon..........1-64 oz., $\$ 2.00 \ldots$ Bibmat ...

Gracilis Prima Donna......1-64 oz., \$2.50.. Bibozita ..

Rex, extra fine mx. pkt. (500 seeds) 20 c net.. Bibola ...

Veruon, splendid bedding sort, $1 / 8$ oz., $30 \mathrm{c} \ldots$ Bibrax $\ldots 2.00$

Bellis, or Double Daisy, White......................

Vaughan's Mammoth Mixturo... $1 / 8$ oz., $25 \mathrm{c}$. . Bifrons ...

" White ... W oz., 30c..Big .... 1.80

l'ink ....1/8 oz., $30 \mathrm{c} \ldots$ Bigamist .. 1.80

Monstrosa, double white, new..1/8 oz., 60c.. Bigamy ... 4.00 Double Pink, new.......1/8 oz., $60 \mathrm{c}$.. Biggin ... 4.00

Turban, new, double red....1-32 oz., \$1.80.. Bigbart ...

Longfellow, double plnk......................

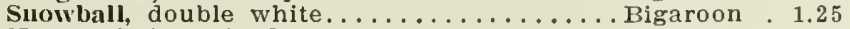

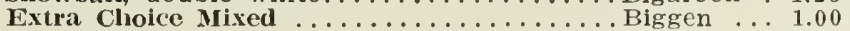

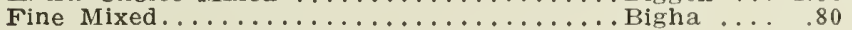

Betonica Grandiflora Superba.......................... 1.25

Bidens Atrosanguinea, the black Dahlia. . .......Bike .... . 40

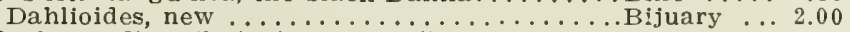

Bird of Paradise, (Poinciana Glllesi) . . . . . . . Bllbo ... . . 30

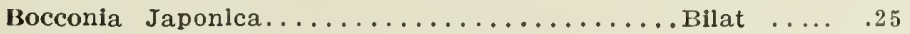

Boltonia Asteroides . . . . . . . . . . . . . Bilamary . 1.20

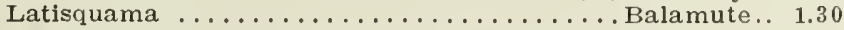

Brachycome Iberidifolia, Swan River Daisy, Blue.Bilalo .... .30

Iberidifolia, White ......................... 30

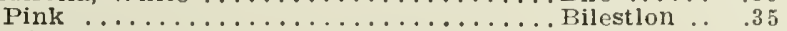

Mixed ..................................

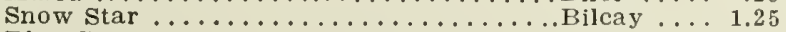

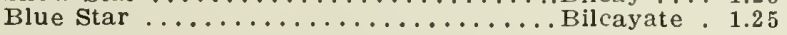


TEL. CIPHER.

Billiards . \$0.10

Bloiza Maxima

Browallia Elata, mixed.

Speciosa Major, large, blue .1-16 oz., \$1.25 . Blllous ..

Bryolupsls Lacinusa, tine annual climber....... Bilirubin ..

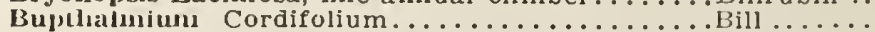

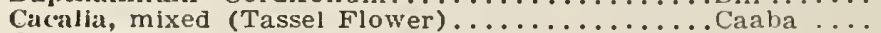

Cactus, mixed

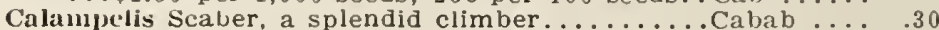

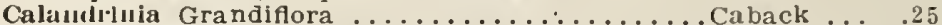

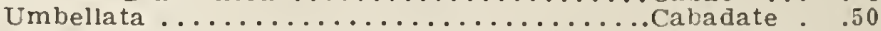

Calccolaria Hybrida, finest strain. $1-32$ oz., $\$ 1.00$. Caballero

Calendula (Pot MIarigold). Meteor..5 lbs., \$2.50..Calasset Sulphurea Plena, double sulphur-yellow..... Cabbish

rrince of Orange ........... lbs., $\$ 2.75 \ldots$ Cabble ...

Grandillora fl. pl. (Örange King) 5 lbs., $\$ 2.75$. Cabeca ...

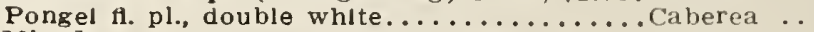

Mixed .......................... $\$ 2.25$. Cabin

Califoruia Poppy, see Escholtzia.

Calliopsis, mixed.............. 1bs., \$2.75..Cabinet ..

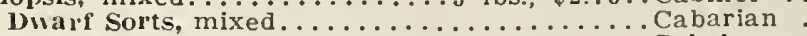

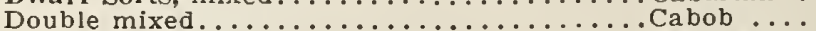

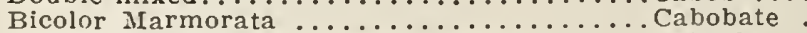

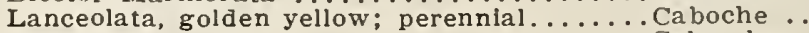

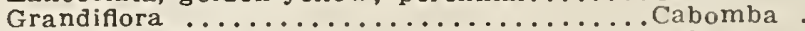

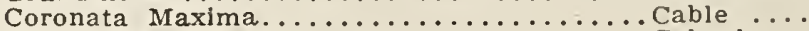

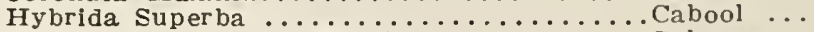

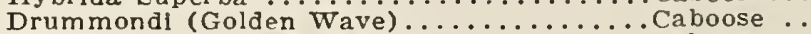

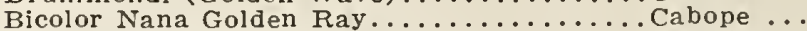

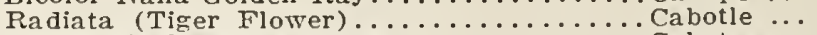

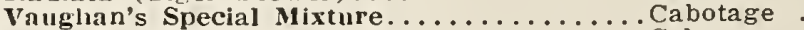

Callirhoe Involucrata (Verticlliata) .........................

CAMPANUIA

Calycanthema, Cup and Saucer, mixed......Cabrank ...

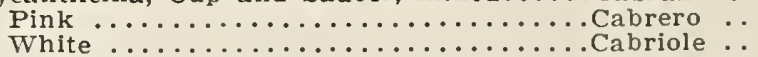

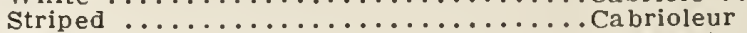

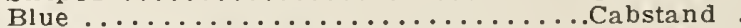

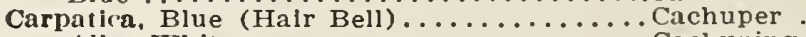

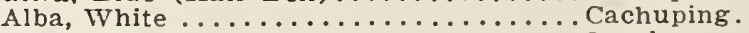

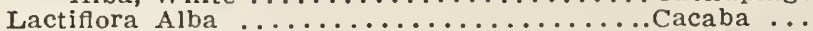

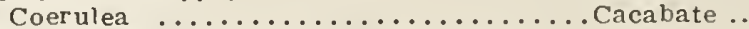

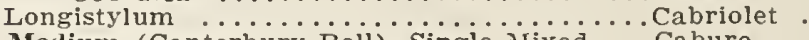

Medium (Canterbury Bell), Single MIixed.... Cabure ...

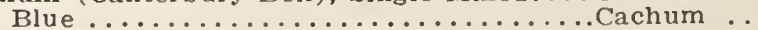

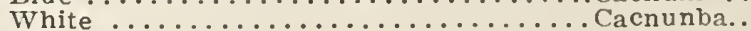

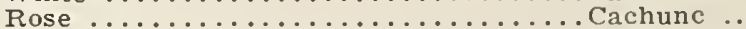

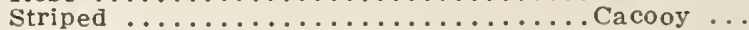

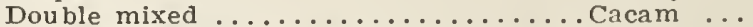

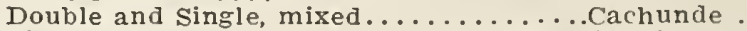

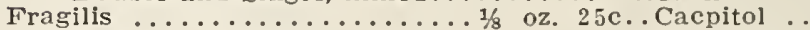

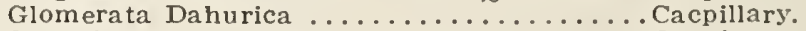

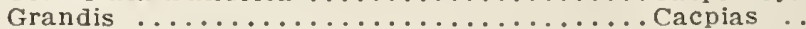

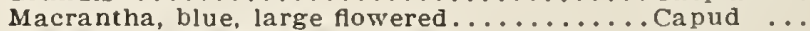

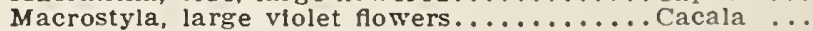

Mirabilis, beautiful species....1-32 oz., 50 c. . Cacadot ..

Speculum (Venus Looking Glass), mixed.... Cachou ...

Perslclfolla Grandifnera Alba (Backhousel) ...Cachexia .. 1.00 Grandiflora Coerulea .................................. 1.00 Double white (Moerheimi) is oz., $\$ 1.50$. Caclam ...

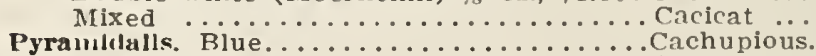

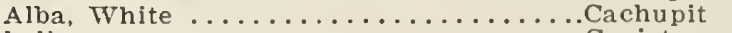

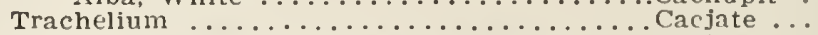

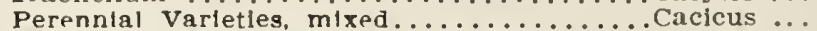

Canary Blrd Flower, fine climber for shade.............. oz.

$\mathbf{L B}$

$\$ 0.70$

2.50

2.50

1. 50

3.50

10

.10

.10

.10

.10

.10

.10

.10

.10

.10

.20

.30

.20

.15

.10

.15

.20

.20

.55

.60

.60

.60

1.20

.50

.60

.80

1.00

1.00

2.00

3.50

2.00

1.60

.60

1.80

2.00

2.00

.50

1.00

1.00

1.00

. 50

.25

.25

2.00

2.60

1.00

.15

.20

.20

.20

.20

.40

.25

1.30

2.00

2.20

2.20

2.20

4.00

3.00

2.00

1.50

.20

. 50

.10

1.00

1.00

.40

.40

.40

.30

.20 


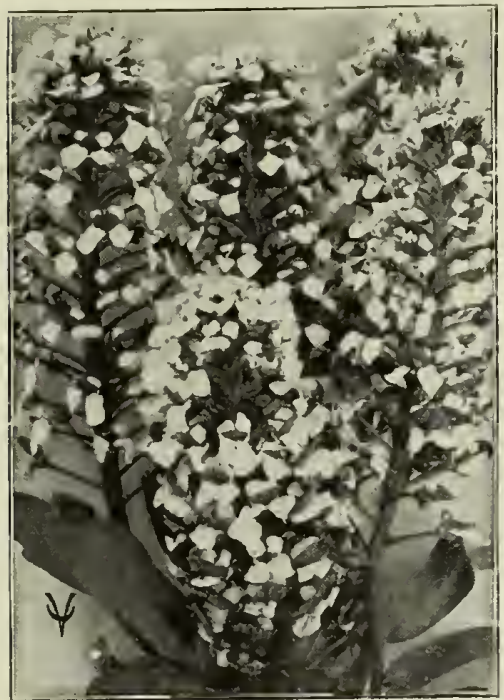

CANTYTLFT EMPRESS

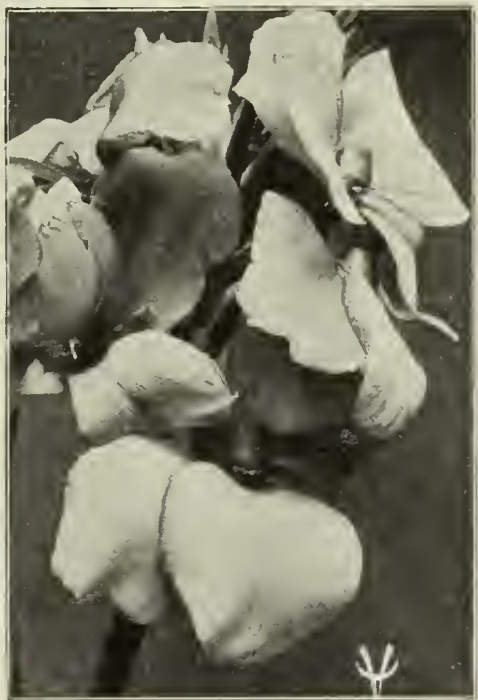

CANNA Vaughan's Glant Flowered

\section{CANDYTUFT.}

TEL. CIPHER.

oz.

LB.

Candytuft. Coronaria. White Rocket, 5 lbs. $\$ 1.75 \ldots$ Caco . . $\$ 0.10$ Coronaria "Princeps" (Llttle Prince).......Cagebird .. .20

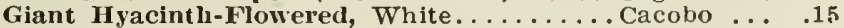
Eupress, selected stock .......................40 Crimson, (Umbeliata Purpurea) ....................... . . . . . . .

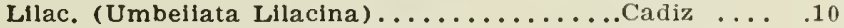
Carmine, (Umbellata Carminea) .................... . 15 Queen of Italy, pink, dwarf, selected stock..Codong ... .30

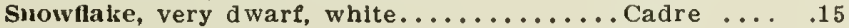

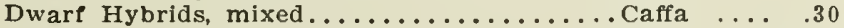
All Color's Mixer, 10 ibs., $\$ 4.00 ; 51$ bs., $\$ 2.50 \ldots$ Caftan $\ldots . .10$

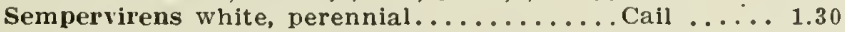

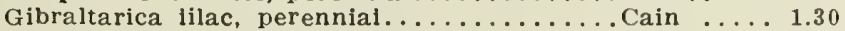

Canna, Crozy's, best mixed .......5 lbs., \$5.75 . Cakus ... . 10

Ci*zy's, Vauglian's special Mixture, saved

from our own collection of hest 50 kinds... Cajoling .. $\quad .15$ $\$ 0.40$ 2.00 1.60 4.00 .85 1. 20 1.60 3.00 1.80 3.00 .55

Cardinal Climber..........500 seeds, $\$ 1.25 \ldots$ Carrara ...

\section{CARNATION.}

We call special attention to our Carnation Seed. which is raised by specialists.

Vienna Dwarf, extra cholce mixed......... Calf .... .65

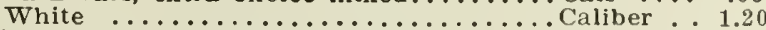

Guiliaud or Giants of Nice, $\mathrm{mx} 1-16$ oz., $\$ 2.00$.Caigar ....

Double Perpetual (Chabaud), mxd......... Calico ... 1.50 Marie Chabaud, yellow ............................ 3.00

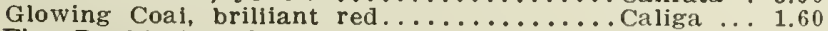
Fine Double Mixed............................. 6 .

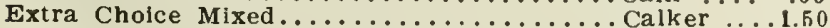
Grenadin. double red, haif high type.......... Camel ... 1.00 Double white ................................. 1.20 


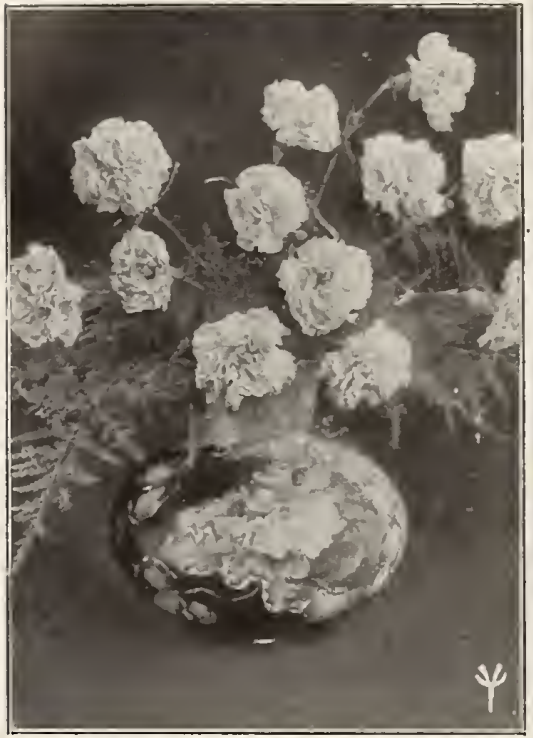

CARNATION Vaughan's Special Mixture

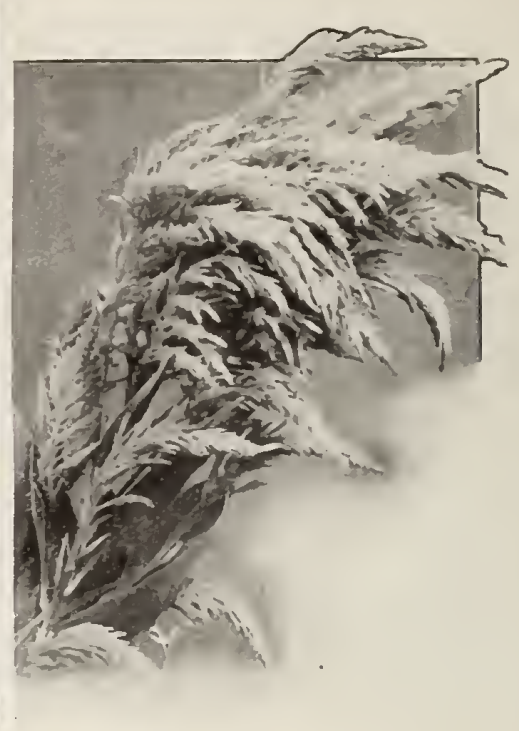

CELosiA Thompsont Magniflea

\section{CARNATIONS-Continued.}

TEL. CIPHER.

oz. LB.

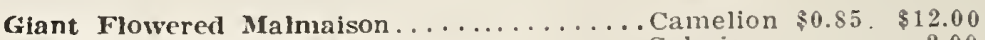

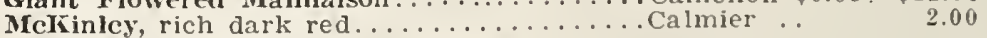

Anlerican Hybrids in finest mixture,

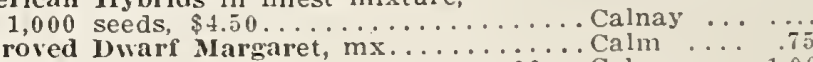

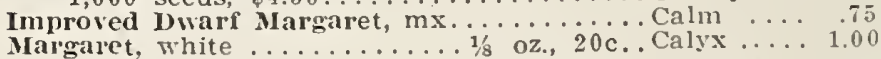

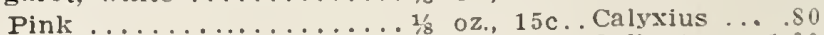

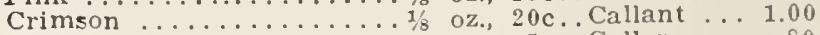

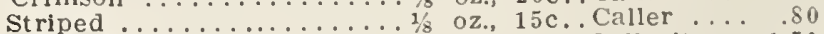

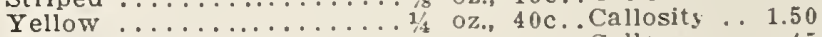

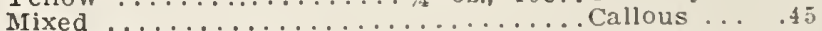

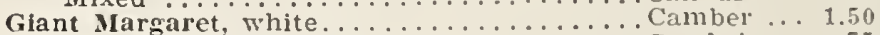

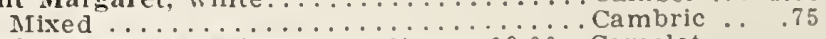

Vaughan's Special iixtule... . is oz. $\$ 3.00 \ldots$ Camelot ..

Cardlospermum, see Balloon Vine.

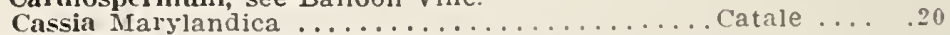

$\ddot{0} \ddot{0} 0$

Castor Oil Bean, see Ricinus.

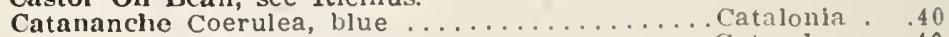

Coerulea Alba, white................................... 40

Catchfly, See Silene Armeria.

\section{CELOSIA.}

Cristata, (Coxcomb) Tall sorts mixed....... Canera .. .20

Cristata Nana. Dwarf sorts mixed, cx. choice.Cames ... 1.25

\begin{tabular}{|c|c|c|}
\hline 18 & "10: & good mixed..... Camomlal. \\
\hline 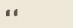 & " & Plesident Tlificl's, true...... Camp..... \\
\hline "1 & 6 & $\begin{array}{l}\text { Enupless, extra choice, dwarf, } \\
\text { crimson combs ......... Campalgn.. }\end{array}$ \\
\hline “ & " & Aurea, dwarf. yellow combs. Campana. \\
\hline “ & “ & Rosen, dwarf, rose combs.. Campaned. \\
\hline " & “ & Fire líng, new. 1/s oz., $45 \mathrm{c}$. . Campanga \\
\hline
\end{tabular}


CENTAUIRA-Continued.

Celestial

Lb.

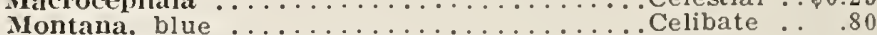

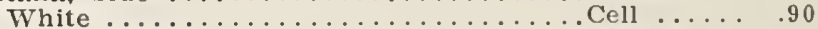

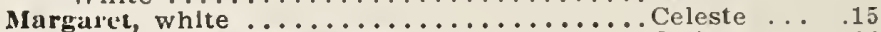

Moscllata, mixed, Sweet Sultan......................... .20

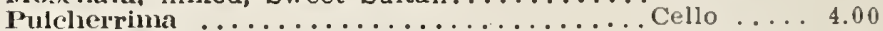

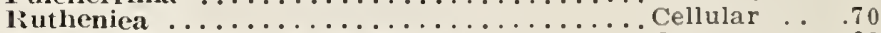

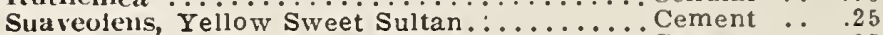

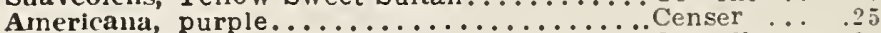

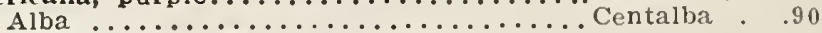

Vaughan's Special Hixture includes all the

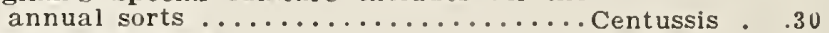

Centrosema Grandlfora ..................... .50

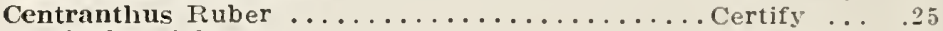

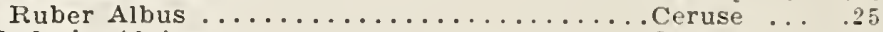

Cephalaria Alpina .............................. 40

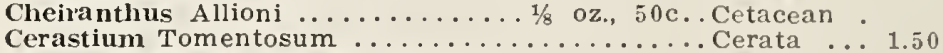

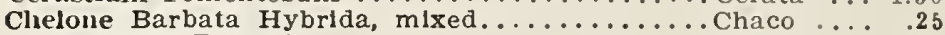

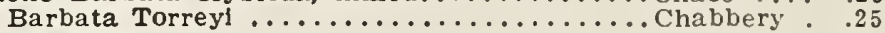

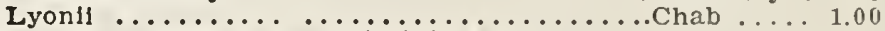

Cheiranthus Maritimus, see Virglnia Stocks.

\section{CHRYSANTHEMUM.}

Coronarium. Double white..................... . . 10

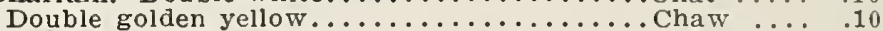

Double sulphur yellow......................... .10

Double mixed............5 lbs., \$3.25..Ched ..... .10

Pumilum Luteum Pl., Golden Ball.......... Century .. .40

$\$ 1.80$

2.00

3.00

12.00

6.00

Dunnetti Album pl., double white................. 35

Aureum pl., double yellow..................... . 30

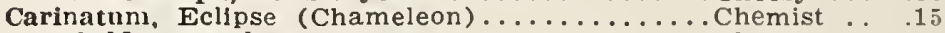

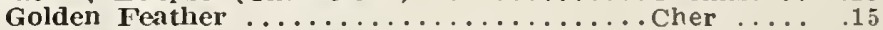

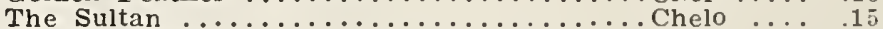

1.80

1.811

1.80

Single Mixed $\ldots \ldots \ldots \ldots \ldots \ldots \ldots \ldots \ldots \ldots$ Cheroot ...

Inodorum Plenissimum, double white..................

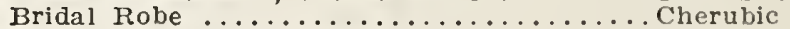

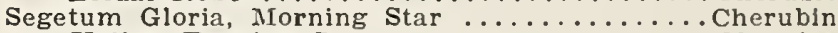

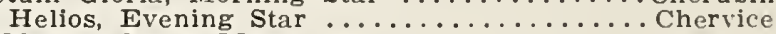

Double and Single Mixed, annual sorts ......... Chess

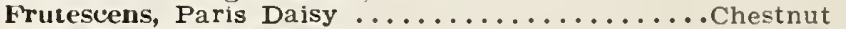

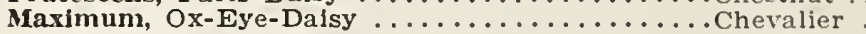

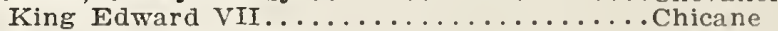

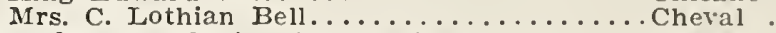

Lencanthemum, Spring Marguerite..........................

Indieum fl. pl., Chinese varieties, mixed, 1/8 oz., $50 \mathrm{c}$ Chide.

.80

.85

.7c

.15

.30

.90

.10

.10

.20

.25

.20

.40

.80

.25

1.50

3.50

1.00

1.20

2.00

2.50

2.20

Japonicum fl. pl., Japanese varieties, mixed.... Chideny . . 4.00 Early Flowering Single, mixed..1/s oz., 60c.. Chief ... 4.00

\section{CINERARIA HYBRIDA.}

Largo Flowering (Maxima) choicest mlxture. 1-32

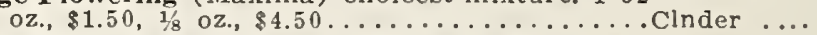
Grandlfora, large-flowered, prize varletles, splendid mixed, $1-32$ oz., $\$ 2.50,1-16$ oz., $\$ 4.50 \ldots$. CIndery ... Vaughan's Columbian Mixture, 1-32 oz., $\$ 2.75,1-16$

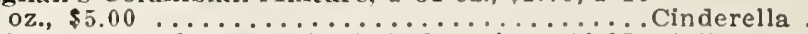
Maxima Nana, cholcest mixed, dwe.,1-16 oz., \$2.25. Cinlld .... Grandiflora, Stella, $1-32$ oz., $\$ 1.75 \ldots \ldots \ldots \ldots$ Cinkefoll ... Large Flowering, sky-blue, $1-32$ oz., \$1.00..... Cinnamole Crimson, $1-32$ oz., $\$ 1.00 \ldots \ldots \ldots \ldots$ Cinnamate Old Rose, $1-32$ oz., $\$ 3.50 \ldots \ldots \ldots \ldots \ldots$ Cinnamic White, $1-32$ oz., $\$ 1.00 \ldots \ldots \ldots \ldots \ldots$ Clnnabar 
CINERARIA HYIBRIDA-Continmed.

oz.

Ls.

Matador, brilliant searlet, 1-32 oz., $\$ 2.50 \ldots \ldots$ Cinerary ..

Ordinary Mixed, $1 / 8$ oz., $60 \mathrm{c} \ldots \ldots \ldots \ldots \ldots \ldots$ Cinque ... $\$ 4.00$

Double, best mixed, 1-32 oz., $\$ 1.00 \ldots \ldots \ldots \ldots$ Cipher ....

Radiata (Polyantha, Stellata) ...1-16 oz., \$1.00 . Ciphering

Cincraria Maritima Candidissima............ Circle .... .20

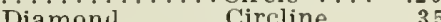

$\$ 2.00$

4.00

Ciarkia Pulchella, Single mixed...................... $10 \quad 1.00$

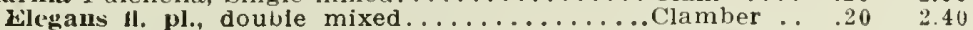

Scarlet Queen ......................... .80

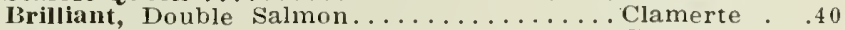

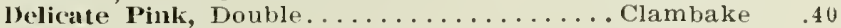

White Prince.................................. .40

Salmon Quecn............................. 40

Clematls Paniculata ......................... . 35

Large F!owering, Jackmani Hybrids, mixed... Clear .... 1.40

Cleome Pungens, Giant Spider Plant, true........ Clease . . . . .15

Clianthus Dampieri, Glory Pea of A ustralla . . . . Cleff . . . . 1.70

Cobaea Scandens, purple............... Coax .... .25

Scandens Flore Albo, white............. Cobalt .... .50

Coccínea Indica, fine climber............................ .60

Cockscomb, see Celosia cristata.

Colx Laclurymae, Job's Tears, write for price on larger lots ............................ .10

Coleus, Vaughan's Rainbow Mixt., 1/8 oz., \$1.50 . Cobby . . . 10.00

Fine Mlixed ................................... . . 1.50

Extra Choice, large leaved, mix, 1/8 oz., \$1.00. Cobweb . 7.00

Lacinated and Fringed.....1-16 oz., $\$ 1.00 \ldots$ Cocoa

Cordyllne, see Dracaena.

Collinsia, mixed .............5 lbs., \$1.60..Cockade .. .10

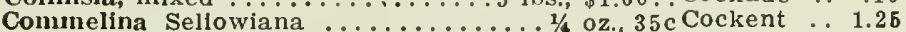

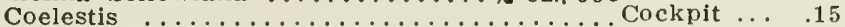

Convolvulus Major (Morning Glory), white....... Cocoon ... .10

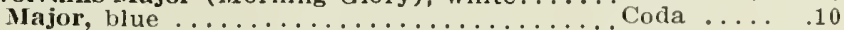

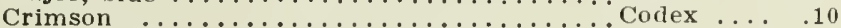

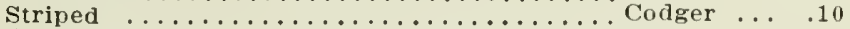

All colors mixed, 100 lbs., $\$ 30,00 ; 10$ lbs..

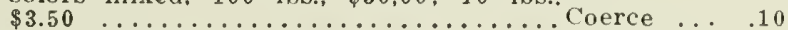

20.00

Imported Collection of 8 varieties,ea, $18 \mathrm{c}$ net. Coercing.

Major, double mixed.................. Coercible . .20

Double white, snow Fairy............................. 45

Imperial Japanese, mixed. . ..5 lbs., $\$ 3.00 \ldots$ Coffer .... .10

Fancy Fringed, best.....5 lbs., \$7.00.. Cofringed... .15

Minor, Dwarf Glory, mixed....5 lbs., \$1.75 . . Cognate . . .10

Monstrosus ........................... 10

Mauritanicus, for hanging baskets............. Cognize ... 30

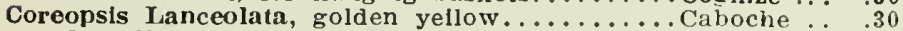

Grandiflora, large flowering, golden yellow... Codak .... .20

Eldorado ...............................60

cosmidium Burridgcanum, orange crown........ Coherer .. $\quad .40$

Cosmos, Glant-flowered, choice mixed........................... $\quad 10$

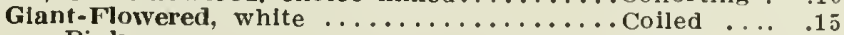

Pink ................................

Crimson $\ldots \ldots \ldots \ldots \ldots \ldots \ldots$ Code $\ldots \ldots \ldots .15$

Lady Lennox, pink...................

Lady Lennox, white .....................

Striped ............................... $\quad .20$

Early Flowering Irybrids, mixed ............ Collapse ... . .20

Dawn, white ............................

Plnk .............................. .20

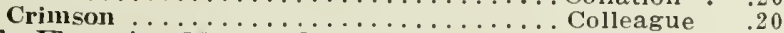

Early Fowering Mammoth, white................. . .

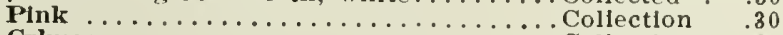

Crlmson . . . . . . $.30 \ldots \ldots \ldots \ldots$ Collectlve.

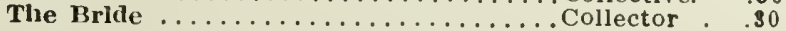




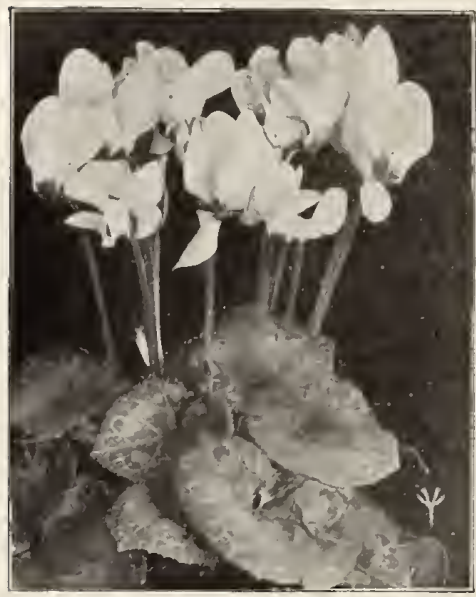

CYCLAMEN

COsMOS, Continued

Klondyke, yellow

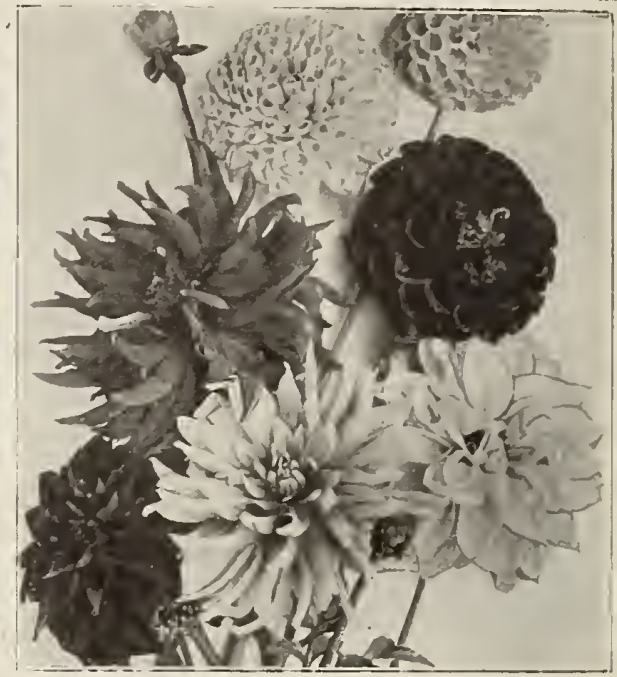

DAHLIA-Vaughan's SDeclal Mlixture TEL. CIPIIEH.

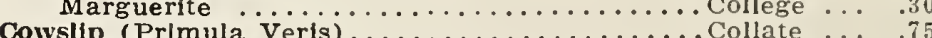

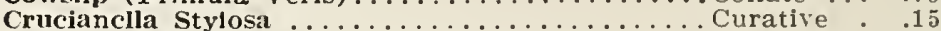

Cucumls Metuliferus, Horned African Cucumber. . Curator . . .40

Cupleea Purpurea ........................... .25

Platycentra............. i oz. $_{8} 60 \mathrm{c}$.. Curate ... 4.00

\section{CYCLAMEN'PERSICUM.}

Fine Mixed .................................. 1.0014 .00

Giganteum, pure white, 1,000 seeds, $\$ 3.75$,

per $1 / 8$ oz., $\$ 1.25 \ldots \ldots \ldots \ldots \ldots \ldots \ldots$ Cycllde $\ldots \ldots$

Rose Marienthal, 1,000 seeds, $\$ 3.75$,

$1 / 8$ oz., $\$ 1.25 \ldots \ldots \ldots \ldots \ldots \ldots$ Cyelone ...

Dark Crimson, 1,000 seeds, $\$ 3.50$,

$1 / 8$ oz., $\$ 1.25 \ldots \ldots \ldots \ldots \ldots \ldots \ldots \ldots \ldots$ Cyclops ...

White with Carmine Eye, 1,000 seeds, $\$ 3.75$

$1 / 8$ oz., $\$ 1.25 \ldots \ldots \ldots \ldots \ldots \ldots \ldots \ldots$ Cygnet ...

Dark Rose, 1,000 Seeds, $\$ 3.75$

$1 / 8$ oz., $\$ 1.25 \ldots \ldots \ldots \ldots \ldots \ldots \ldots \ldots$ Cygnus ...

Mixed, 1,000 seeds, $\$ 1.50 ; 1 / 8$ oz., $70 \mathrm{c} \ldots \ldots$. Cycle ... 4.50

Orchid-Ftowered, mixed .........\$4.50.. Cygnopsis

Pure White, 100 seeds, 65 c..... $5.00 \ldots$ Cylichna ..........

Red, 100 seeds, $65 \mathrm{c} \ldots \ldots \ldots \ldots .5 .00 \ldots$ Cyllchoton $\ldots \ldots \ldots \ldots$

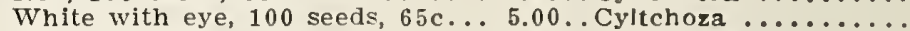

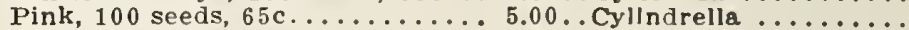

Lilac-Color, 100 seeds, $65 \mathrm{c} \ldots \ldots .5 .00 \ldots$ Cvilndrlcal. .

\section{NEW ENGLISH=GROWN CYCLA MEN.}

\section{TELL. I'TPHER.}

Rosy Morn ......... Czar.

Excelsior ........................

Princess May ........ Czaster.

$$
\text { Any of the above, } 1 / 8 \text { oz. for } \$ 2.00 \text {. }
$$

Salmon King ............. oz., for $\$ 2.25$

Grandiftorum ilbum ............ oz., for $\$ 2.25$

Glory of Wandsbek, per 1,000 seeds, $\$ 7.00 ; 100$ seeds, $\$ 1.00$.

St. George .................. $1 / 8$ oz., for $\$ 4.50$
TEL. CIPRER

Czasth.

. Czarab.

. Czateger

Czyasting.

Czyastic.

\section{Cyat.}

Czestoc. 
TEL. CIPGRR.

น2.

Cyperus Alternifolius, Umbrella Plant......... Cylinder . \$0.60

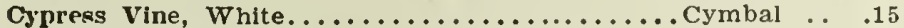

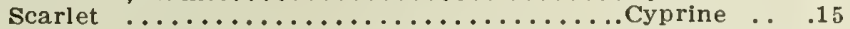

Ivy-Leaved Scarlet ..........................

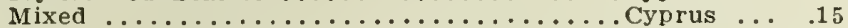

$\$ 1.80$

1.80

1.80

Hybrida Cardinal Climber, 500 seeds, $\$ 1.25$. Carrara .

Dahlia, Double mixed, good quality .......... Dace .... $\quad .50$

Double Peony Flowcred Mixed........... Daelay ... .50

Extra choice mixed............... Dactyl ... 1.00

Cactus-Flowered, mixed .............. Daftness .. .85

Cactus-Flowered, extra choice mixed... Dague .... 3.00

Liliput, mixed ........................ 1.00

Single Giant Perfection, mixed........... Dalmio ... .15

Striped and Spotted .............. Dalle .... .20

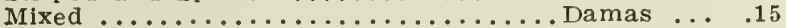

Collarette ............................ . .5

Zimpani (Black Cosmos) ............ Dabble ... .40

20 th Century ..................... 1.00

Colossal, semi-double Peony-flowered,

mixed ................... Dazzle ... .40

Vaughan's Special Mixture, dbl. and sgl.... Dame ... 3:50

Daisy, see Bellis, Agathea, Brachycome and

Chrysanthemum Maximum.

Shasta Daisy, Alaska, best white.......... Dampare .. 1.00

14.00

Shasta Daisy, Ordinary ................ Damascus

Datura Fastuosa fl. pl., double mixed.......... Danaid ...

Double White ..............................15

1.80

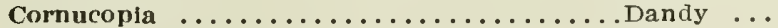

.15

Triple-Flowered yellow (Golden Queen).....Darion .... .15

1.60

1.60

Delphinlum Formosum, blue............... Deacon ... .45

5.00

Formosum Coelestinum, (Pillar of Beauty)... Deadly ...

Belladonua Hybrils ................. Debonair

Chinese Album Grandiflorum, white......... Deflective

Azureum, blue ................ Deflector

Cashmerianum, dark blue; fine.. $1 / 4$ oz., $75 \mathrm{c}$.. Defiexion ..

Ca ucasicum, sky-blue ................. Deflorate...

Nudicaule, red ......................... ozeafen 30 c...

Elatum Iybridum, extra cholce mixed....... Dearth ...

Double hybrids, fine mixed............ Deball ...

Vaughan's Gold Medal Hybrids, an extra

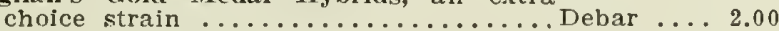

Zalil, yellow, splendid cut flower, $1 / 8$ oz., $40 \mathrm{c} \ldots$ Debet $\ldots .2 .50$

Speciosum Glabratum, blue ............. Defluency . .50

Sibiricum hybridum, blue shades.......................... $\quad .80$

Vauchan's Special Mixture of Pereunial

Varleties ............................. 3.00

See also Larkspur.

\section{DIANTHUS=PINKS.}

Double Chinese Pink, choice mixed, 5 lbs., $\$ 9.00 \ldots$ Digest ... . 20

Single Chinese, extra choice mixed......... Diminish ... .15

Heddewigl f. pl., double Japan, best mixed...... Dimity ... . 35

Single, extra flne mixed............... Dimly ... .25

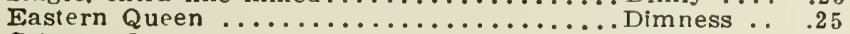

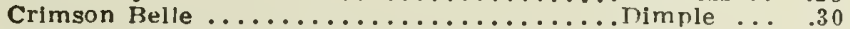

Snow Queen, best double white............ Dinde .... .60

Mtourulug Cloak, double................. Diner .... .50

2.50

3.00

6.50

Dladeniatus f. pl., (Dladem Pink) double... Dingy .... $.35 \quad 4.00$ 
DIANTHCS-Continued.

Laciniatus fl. pl., Double fringed, mixed.... Dining . .\$0.50

TEL. CIPHER. OZ.
Dining ...\$0.50

Lucifer fl. pl $\ldots \ldots \ldots \ldots \ldots$ Dinopisky .1 .00

Vesuvius, orange scarlet........... Dingle ... . .35

Mephisto, flaked and striped .......... Dintatler. .35

Mirabilis, very fine................ Dinsure .. .35

Mirabilis $\mathrm{f}$. pl. dbl. nixd........... Disartge . . 1.00

Nanus Conıpactus, F'ulry Quierll...... Dijon .... $\$ 0$

Albus Pl., Snowdrift............ Dinopls .. .60

Double Striped ................. Diplaz . . .75

Midnight, double blood-red............... Diobol .... . 50

The Bride, single white............. Dlorite ... 30

Count Kerchove, dark blood red.......... Dinoplste. . .50

Snowball, dwarf ................. Dipopa ... 50

Fireball, dwarf ................ Dipper ... 85

Marginatus, sllver-edged ............ Diplomate .35

Superbissima Mixed ............... Displaxy . . 25

Double Imperial Pinks (Imperialis fl. pl.), mixed. Disable . . 25

New Princess Pink, very fine.............. Disabuse . . .35

Hybridus Latifolius Atrococcineus pl ......... Dirge . . . .45.

Vaughan's Special Mixed, Dbl. and Single Pinks. . Disarm ... .50

Vaughan's Special Mixture, Single Pinks........ Disband .. .40

Vaughan's Special Mixture, Double Pinks....... Disbar . . . .50

Plumarius, Single Pheasant's Eye Pink......... Discard . . 15

New Early Flowering, double............ Discount $\ldots 1.50$

Fl. Pl., double mixed.............. Disclose .. .85

Albus Plenus .......................

Cyciops ....................... 50

Semperflorens, double and single mxd...... Disc .... .90

Diadematus ...................... Discomb .. 1.00

Vaughan's Speclal mixture ............ Discombed. 1.25

Barbatus, see Sweet William.

Dictamnus Fraxinclla, Gas Plant........... Pistrain . . .15

Fraxinella Alba ................. Dack .... 15

Caucasicus ......................... 50

Didiscus Coeruleus, blue............... Doctering . .80

Digitalis (Foxglove) Purpurea Monstrosa, mixed. Dissintory. $\quad .30$

Purpurea, mixed ................. Dissent ... .15

Gloxiniaeflora, mixed ............... Dissenter . .15

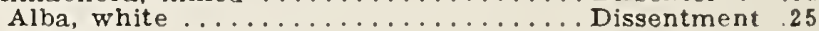

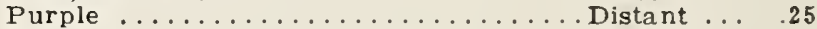

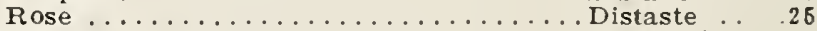

Grandiflora yellow ............... Dissoclal . 10

Lanata ..................... Disspated . 10

Maculata Iveryana, spotted varieties....... Dissuade . .25

Vaughan's Special Mixture. . . . . . . . . Distent . . . 35

Dimorphotheca Aurantiaca, Orange Daisy....... Dividend .. .50

New Hybrids .................... Divlder ... 80

Dolichos (Hyacinth Bean), mixed.......... Doctor ... .10

Princess Helen (Daylight) ............. Doctrine .. 10

Purple Soudan .................. Dolent ... 10

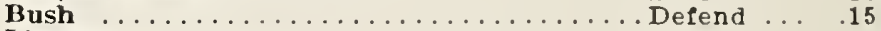

LIenosus ..................... Delhi ... .50

Dracaena Indivisa Lineata ............... Draegle ... .30 3.00

Indivisa ..................... Draln ... $20 \quad 2.00$

Indivisa Latifolla .................. Draisine ... 30

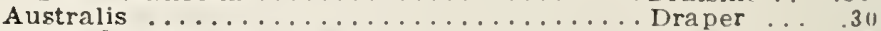

Dracocephalum Moldavieum ............. Dresden ... in

LB.

$\$ 5.00$

2.60

4.00

4.00

4.00

\$. .00

3.00

6.00

10.00

5.00

3.00

2.60

4.00

5.00

6.00

4.50

6.00

1.80

12.00

6. 00

1.5

1.50

3.50

1.50

1.50

2.40

2.40

2.40

1.00

2.411

4.00

B. 00

in

1.00

1.00

1.80

3.00
2.00

.40

Echeveria (Hen and Chlcken), mxd., 100 seeds

for $15 \mathrm{c} ; 1,000$ seeds, $90 \mathrm{c} \ldots \ldots \ldots \ldots$. $\ldots \ldots$ chas

Metallica, 100 seeds, $25 \mathrm{c} ; 1.000$ seeds, $\$ 2.00$. Filatior

Secunda Glauca, 100 seeds for $20 \mathrm{c}: 1,000$

seeds, $\$ 1.80 \ldots \ldots \ldots \ldots \ldots$. . . . . . . . . . . .

ne smetlona, 100 apeds, isc; 1,000 seads, si. on Flate 
TEL, CIPARR.

oz.

L.

Echlnacea Hybrida, beautiful new varleties..... Echate . . \$1.00 Purpurea ................................. 1.00

Echinocystis Lobata, Wild Cucumber, 5 lbs., $\$ 2.50$. Echinoc . . $\quad .10$

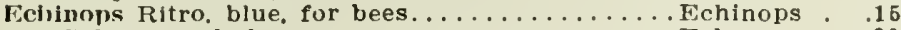

$\$ 0.65$

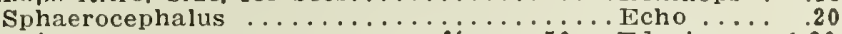

Edelweiss ..............per $1 / 4$ oz, $50 \mathrm{c} \ldots$ Edenic ... 1.80

Frigeron Aurantiacus Hybridus... 1/8 oz., \$1.25 . Enlgma ...

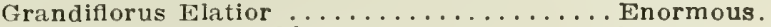

Erynglum Planum, dark blue.................... .15

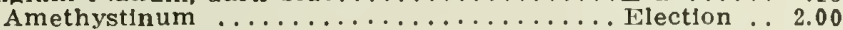

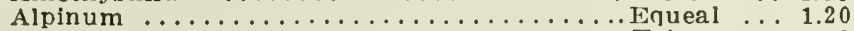

Frysimum Arkansanum .............. Erie ... .10

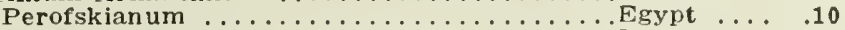

Erytlurina Crista Galı, Coral Tree................... .50

Escholtzia, Californla Poppy, Mandarin........... Eruption ... $\quad .15$ Californica, pure yellow .....5 lbs., $\$ 3.50 \ldots$ Eregsiple . .10

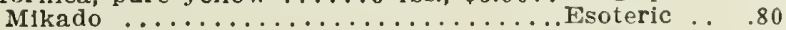

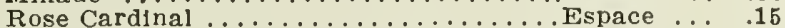
Rosy Morn, Canaliculata Rosea.........Essay .... .20 Glory of the West, or the Golden West. . Espoir ... . .15

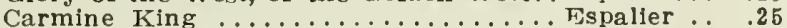

Maritima, Maltese Cross .............. Espohr .. .15

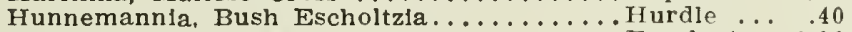

Erceta Compacta Dainty Queen........................ . 1.00

Mixed, many colors.........5 ibs., $\$ 3.25 \ldots$ Estate .... 10

Vaughan's Special Mixture ...................... 30

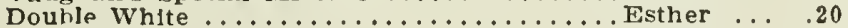

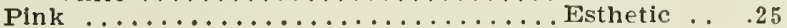

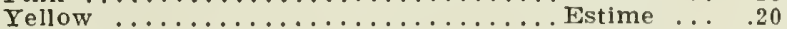

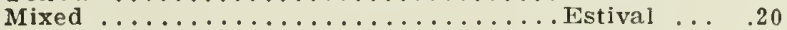

Euealyptus Globulus, Blue Gum............. Evylin ... . 30

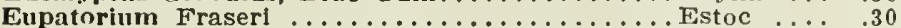

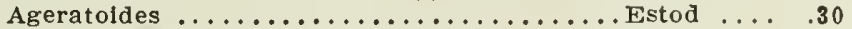

Euphorbla Heterophylla, Mexican Fire Plant..... Euphema . $\quad .35$

Variegata, Snow-on-the-Mountain ........ Eva ..... 10

1.80

1.80

.80

1.60

2.20

1.50

2.80

1.80

4.50

Everlastings, Mixed, many kinds.

Evelina

.15

4.00

1.20

1.40

Everlasting Fowers. see Ammobium, Hellchrysum

Acroclineum, Rhodanthe, Xeranthemum and

Gomphrena

Feverfew. see Matricaria and Pyrethrum.

Forget-Me-Not, see Myosotis

Four O'Clock, see Marvel of Peru.

Foxglove, see Digitalis.

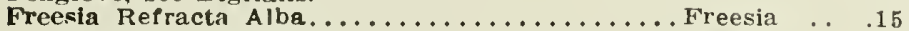

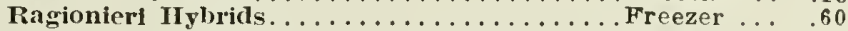

1.60

Fuchsla. Dbl. and single $m x d, 1,000$ seeds, $\$ 2.50$ Fuchs .... Double mixed .........1,000 seeds, $\$ 2.75$. Fugltive ... Single mixed ........ 1,000 seeds, $\$ 2.40 \ldots$ Fugacious: Procumbens ...........1,000 seeds, 75 c.. Flight ....

Gaillardia Lorenziana, double mixed..........Gainer ... $\mathbf{. 1 5}$

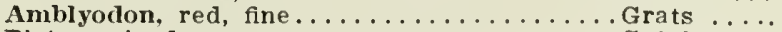

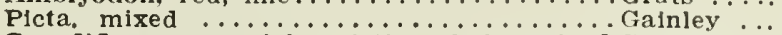

Grandifiora. perennlal varletles, cholce mixed.Gainsaier . Campacta, dwarf sorts ............. Gainsay

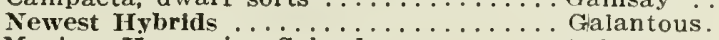

Maxima Kermesina spiendens........... Galantery

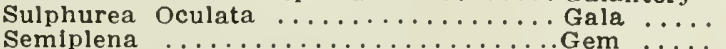

Gaura Lindheimeri, white, very fine............ Gesulate . 16

Gazania, Hybrids, mixed......100 seeds, $60 \mathrm{c} \ldots$ Gild ..... Gentiana Acaulis, dark blue, fine.................. . .50

Geranium, Apple-scented, 100 seeds, $25 \mathrm{c} ; 1,000$ speds, $\$ 1.50 \ldots \ldots \ldots \ldots \ldots \ldots \ldots$. Generosity. 
GERANIUM-Continued.

Zonale single, fine mixed. ................. Generous $\$ \$ 0.65$

LB.

Single, extra, large-flowering, mixed.... Genepa ... 1.00

Lady Washington, 1,000 seeds, $\$ 7.00 ; 100,90 \mathrm{c} G$ eneru

Double Mixed, 1,000 seeds, $\$ 3.00 \ldots \ldots$ Genoa

Gerbcra Jamesoni Hybrids. $\ldots \ldots \ldots \ldots$ per 1,000 seeds. Genlal

Geum Atrosanguineum Pl., semi-double, red..... Genesis ... .50

Heldreichi, large orange flowers.......... Gold .... 1.00

Gilia, mixed ................. ibs., $\$ 1.75 \ldots$ Gilding ... .10

Gilly Flower, see Stocks.

Gladiolus Praecox, annual gladiolus.......... Glade ... 1.50

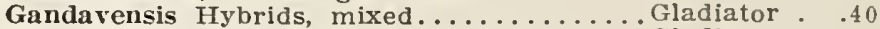

Primulinus Hybrids.................................. 1.50

Glaucium Luteum (Horn Poppy) ...........Gladden .. .10

Globe Anıranth (Gomphrena) mixed .......... Gloher .... .10

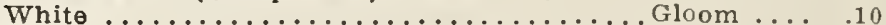

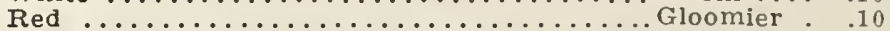

1.00

.90

1.00

1.00

Gloxilla Hybrida Grandiflora, best strains In finest mixture .........1-32 oz., \$1.25..Glossom

Fine mixed................ $1-32$ oz., $75 \mathrm{c}$. Glosam...

Godetia, many sorts mixed........................... .

Duchess of Albany .......................... . .

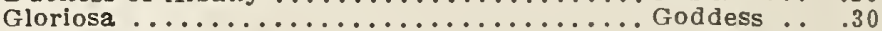

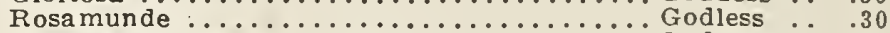

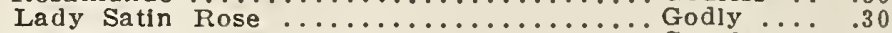

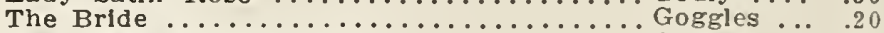

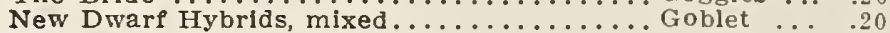

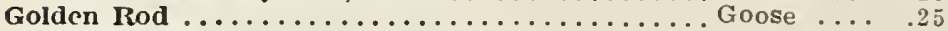

Gomphrena, see Globe Amaranth.

Gourts, Dish-Cloth ......................... . 10

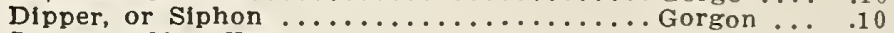

Japanese Nest Egg .............................. .10

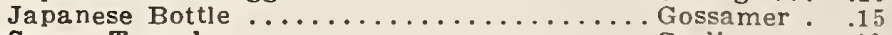

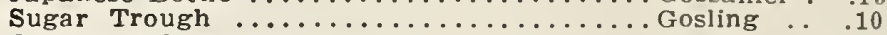

Ornamental Pomegranate .................... .10

Hercules Club ............................. . . . .

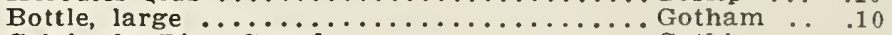

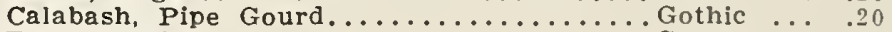

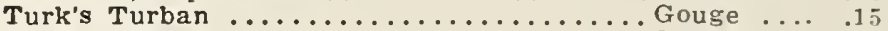

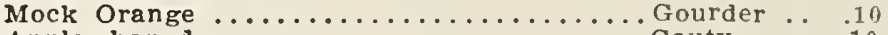

Apple-shaped ............................. . 10

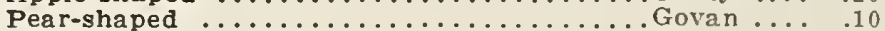

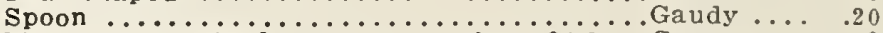

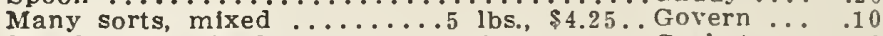

Small sorts, mixed........... lbs., $\$ 4.50 \ldots$ Grabat $\ldots . .10$

Imported Collection of 6 var., ea. 12 c...... Grabble

12 var., ea. 25 c......Graceful

Grasses, Ornamental

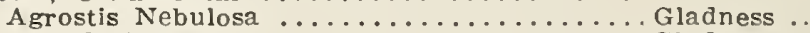

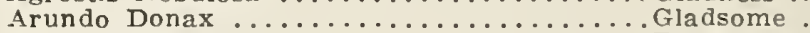

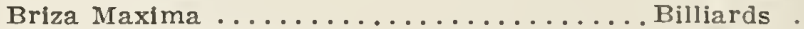

.10

.10

.70

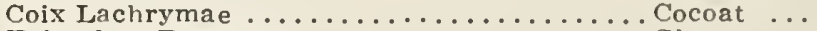

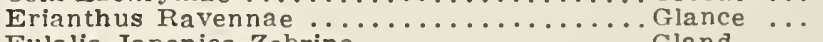

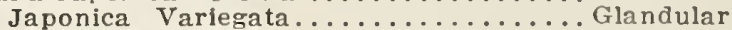

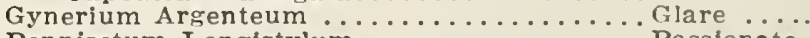

.25

.20

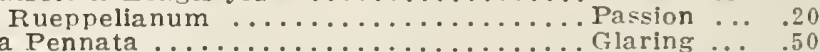

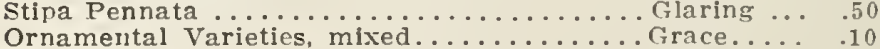


GYPSOPHILA-Continued.

TEL. CIPHER.

oz.

LB.

Elegans. white (Angel's Breath) $10 \mathrm{fbs}, \$ 3.00$. Gyrate . . \$0.10

Rosea ................................ .10

Alba Grandiflora........ 5lbs., $\$ 2.25 \ldots$ Gyzat $\ldots . .10$

$\$ 0.35$

.40

Carminea.

Gyral

50

.80

Helenfum Autumnale Superbum, tali goiden yeliow Heat . . . 1.80

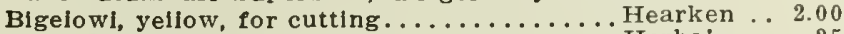

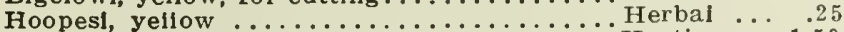

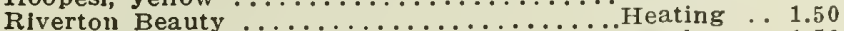

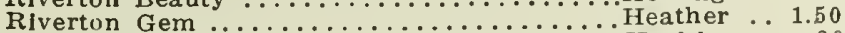

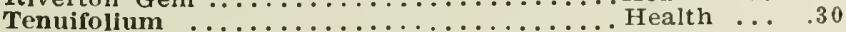

Helianthus, see Sunfiower.

Hcliclirysum Monstrosum f. pl., double mixed... Healer ... .25

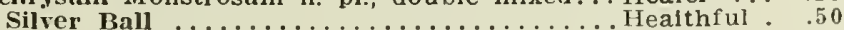

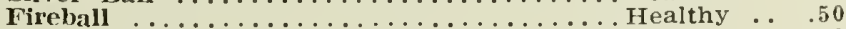

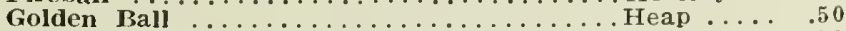

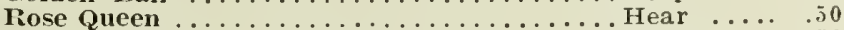

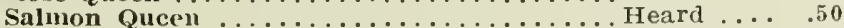

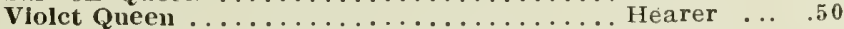

Heliopsis Pitcheriana, goiden yellow, early.....Height ... .25

Helipterum Sandfordi ......................... .25

Heliotrope, fine mixed..........1/4 oz., 20c.. Hearty ... .65

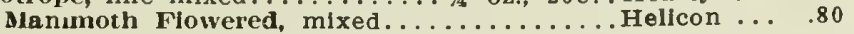

8.00

11.00

Hesperis Matronalis (Sweet Rocket)...........Helmet ... .15

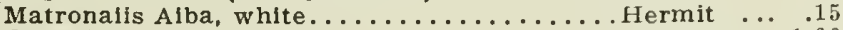

1.25

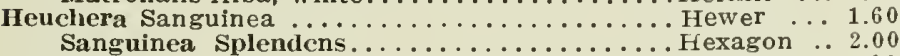

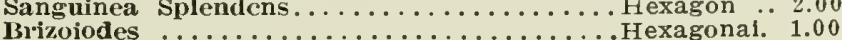

Hibiscus, Glant Yellow or Sunset (Goiden Bowi).. Hiatus .... .35

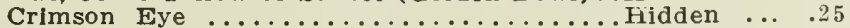

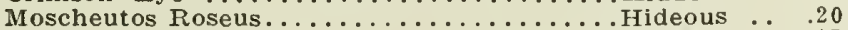

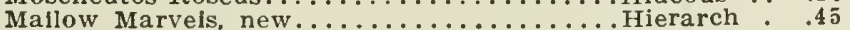

Hollyliock, Double White.................Hoery .... 1.00

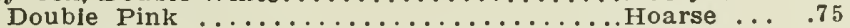

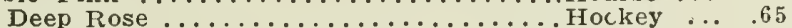

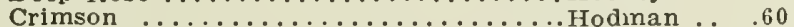

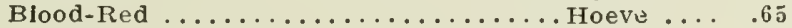

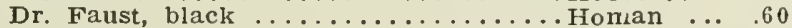

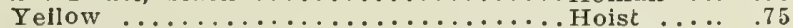

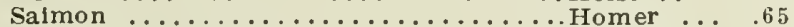

White, pink center ............................... .75

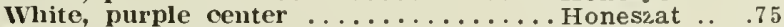

Mixed, extra choice (Chaters) ............ Homily ... .85

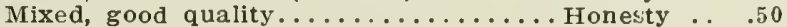

2.50

1.25

4.00

2.50

2.40

5.00

9.00

8.00

7.00

8.00

7.00

9.00

8.00

9.00

9.00

6.00

Single Black ............................... 1.00

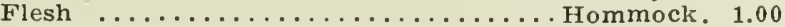

Pink .............................. 1.00

Red ...................... Honestiy . 1.00

Rose ........................ Honey ... 1.00

White ................................... 1.00

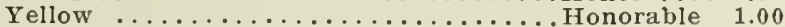

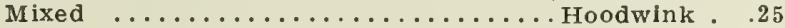

2.50

Mammoth Alfegheny, best mixed.......... Hoof .... .50

Everblooming, single mixed............. Hoofing ... .30

Doubie Annual, mixed .......................40

6.00

3.50

4.50

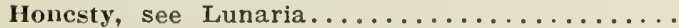

Horned African Cucumber................. Hoofhorn . .20

2.00

Humulus Japonicus, Japanese Hop...........Hook .... . .15

1.80

Japonicus fol. var., variegated........... Hoop .... .20

2.00

Hunnemannia Fumariaefolla, Bush Escholtzia... Hurdle ... .40

4.50

Hyacinth Bean, see Dolichos.

Hyacinthus Candicans ....................... 
Ice Plant (Mesembryanthemum Crystallinum) ... Iceland . $\$ 0.10$

Impatiens Sultani, brill't carmine, 1-16 oz., 70c..Image ... 8.00

Holsti, very fine..........1-16 oz., 60c.. Imitate ... T.00 New hybrids ..........1-16 oz., 40c..Imitator ..

Incarvillea Delavayi ............1/8 oz., $25 \mathrm{c} \ldots$. Incar ... 1.60

Granditlora ..................... Imagery .. 1.80

Inula Ensifolia, golden yellow, dwarf......... Idol ...... 90)

Glandulosa Grandiflora .......1/8 oz., $60 \mathrm{c}$. Idler ....

Ionopsidlum Acaule, Diamond Flower......... Iono .... 1.25

Ipomoea Noctifora, Moonflower, white seeded.... Ipecac ... 30

Noctiflora, black seeded.............. Ipfam .... .25

Hybrid, Moonflower, early blooming.... Iphisa .... . 30

Glant Pink (Northern Light) ........... Ipjar ... . 20

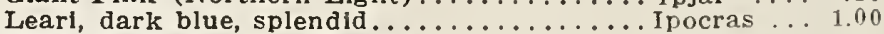

Bona Nox, large blue flowers............... Ireful .... 10

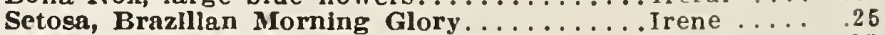

Rubra-cœrulea, or Heavenly-Blue ......... Iridal ... .25

Early Flowering.................. Irish .... .75

Imperialls, Japanese Morning Glory

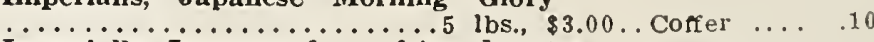

Imperialls, Japanese fancy fringed, extra

$\ldots \ldots \ldots \ldots \ldots \ldots \ldots \ldots \ldots . \ldots$ lbs., $\$ 7.00 \ldots$ Cofringed . . 16

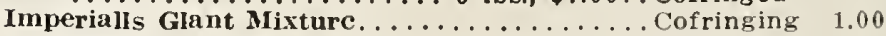

Rochester Morning Glory........................... 15

Coccinea, scarlet, very free................. Incldent .. 10

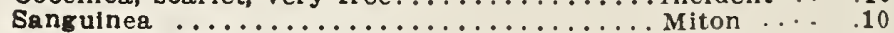

Iris Kaempferi, Japanese Iris.............. Iris . . . . .25

3.50

2.80

3.00

2.40

.80

2.80

3.00

9.00

Job's Tears, see Coix Lachrymae.............

Kenilworth Ivy, Linarla Cymbalaria, pink.......... . . . . .90

Cymbalarla Alba, whlte..................... .70

Kochia Tricophylla...........

Kudzu Vine, Pueraria Thunbergiana...........Kudzu ... .40

Lantana Hybrida, mixed............................ .

Bruant's Dwarf Hybrids, mixed............ Laflin .... .20

Larkspur, Emperor, extra choice mlxed......................... 10

Double Dware Rocket, best mixed............. Lambeau . 10

Double Tall Rocket, best mlxed............................ . 10

Double Giant Hyacinth-Flowered, mixed...... Lambrock. 10

Stock Flowered, White................................ . 20

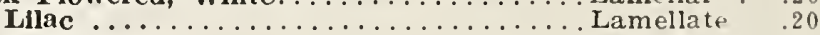

Dark Blue ................. Lamina ... .20

Flesh $\ldots \ldots \ldots \ldots \ldots \ldots$ Lammas ... .20

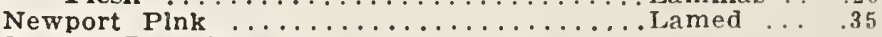

See also Delphlnlum.

Lathyrus Latlfolius (Everlasting Pea), mixed.... Lament ... . 15

Latifollus Albus, white................................. .20

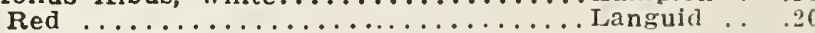

Plnk Beauty, very fine..................... .20

White Pearl ................................... 200

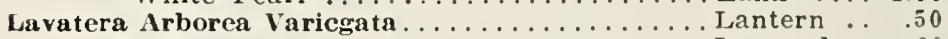

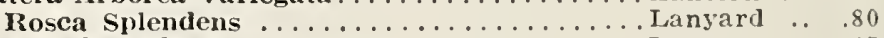

Alba Splcndens .............................. 45

Trimestris Grandiflora Rosea...................... 10

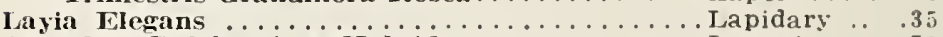

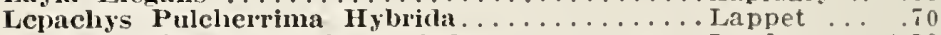

Lemon Verbena. Aloysia Citriodora..................... 1.50

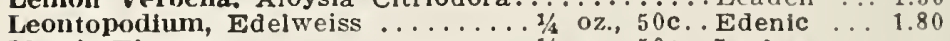

Llatris Elegans................. oz., $50 \mathrm{c}$. . Leakage ..

Splcata .................. oz., $50 \mathrm{c} \ldots$ Leap ....

Linaria Cymbalarla. Kenilworth Ivy........... Lien ..... .90

Cymbalaria, white ...................... . 0 


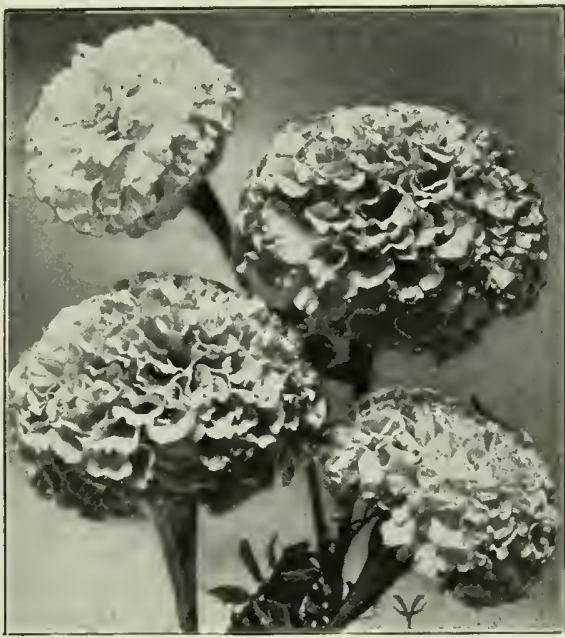

MARIGOLD-Double Tall African, Speclal Mixture

Linuı Grandiflorum Rubruin, Scarlet Flax...... Lifeless . . \$0.10

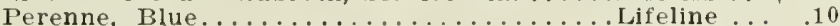

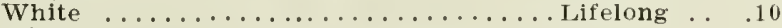

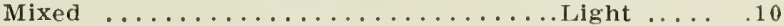

Flavum, perennial, yellow, fine.................... 50

Lobclia Crystal Palace Compacta, true ........ Loading . . .90

Compacta Firnanicnt................ Local .... 1.80

Spcciosa, dark flowers and foliage, true.... Loaf ..... .30

Erinus, Emperor William, dwf., choice strain.L Loamy ... .65

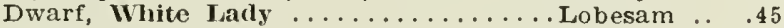

White ............................. .40

Gracilis, blue ........................... . .25

Pumila Splendens (Bedding Queen).... Locate ... .85

Pumila Rosea ...................... 1.00

Mixed ............................... .25

Hamburgia, for hanging baskets, 1/8 0z., 25c.. Loanaye . 2.40

Heterophylla Major ......................... 1.00

Hybrida Sapphire........1-16 oz.. \$1.00 . Lobbaire .

Cardlnalis ..................... Logical ... 1.75

Syphilitica, blue...........

Lunaria Bicnnis (Honesty), dark crimson...... Lop ..... . 20

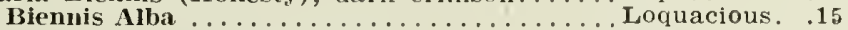

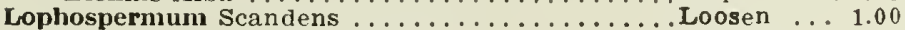

Luffa Acutangula, Dish Rag Gourd....................... 10

Luplnus, Annual sorts mixed, tall....................... .10

Annual sorts mixed, dwarf............. Luncheon . .15

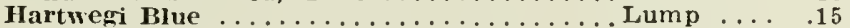

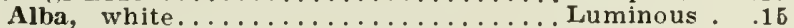

Cruickshanki, dark blue......................... 15

Polyphyllus Albus........................... 15

Atrocoeruleus .......................... .10

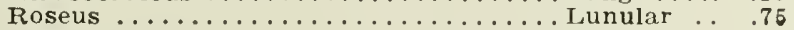

Mixed ............................... . . .

Arboreus snow Queen ............................ 50

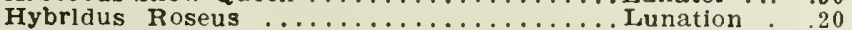

Atrococcineus ................... Luminary . .15

Subcarnosus, blue dwarf...................... 25

I.1s.

$\$ 0.70$

1,40

1.40

1.30

12.00

3.50

8.00

5.00

4.50

3.00

11.00

2.80

20.100

2.00

1.80

1.20

.70

1.40

1.80

1.80

1.60

1.20

9.00

1.00

2.00 
TEL. CIPHER.

Oz.

LB.

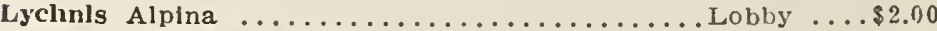

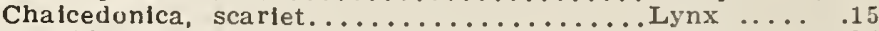

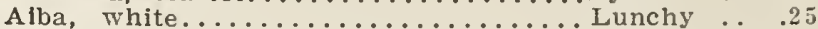

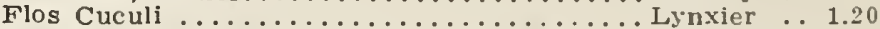

Haageana hybrida mixed, extra choice...... Lyre .... .60

Scarlet ........................................ 1.00

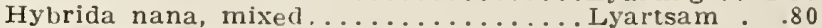

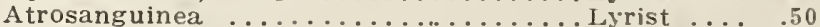

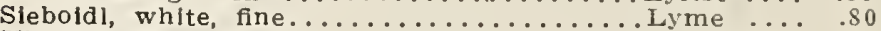

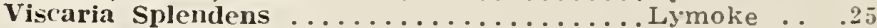

Lythrum Roseum Superbum ................... . . . . . . . . . . . . . . . . . . . . .

Malope Grandiflora Rosea ...................... . .10

$\$ 1.80$

2.50

7.00

1.80

1.00

\section{MARIGOLD.}

Marigold, Double Tall African mixed, 5 lbs., \$8.50. Madame . . .15

Double Tall African, Orange Queen......... Mackerel . .25

Eldorado, a fline strain......................... .20

1.80

Double Dwarf African, mixed, (Pride of

the Garden) ............................ . 20

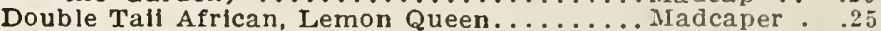

Vaughan's Special Mixture, Tall sorts.............

Double Tall French, mlxed......................... .15

Double Dwarf French, gold striped........... Maimed .. .20

“ Aurora ....................... .25

2.50

2.00

2.00

2.50

2.80

1.80

2.00

2.80

Double Dwarf French, mixed, 5 ibs., $\$ 7.50 \ldots$ Malofic ... $.15 \quad 1.60$

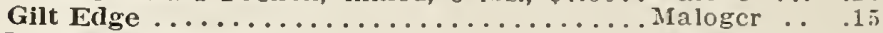

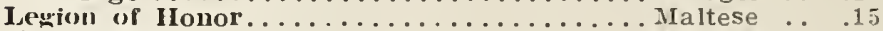

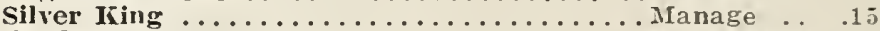

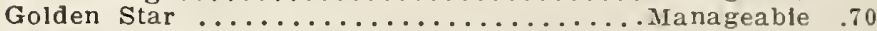

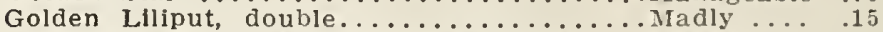

Vaughan's Special Mixture, Dwarf sorts.......Magasin .. .25

1.80

1.80

Imported Collection French Marigold, 6

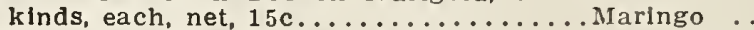

Imported Collection African Marigold, 6

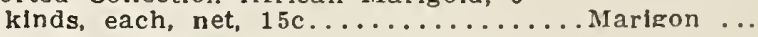

See also Calendula

Marvel of Peru, Four o'clock, mixed. Write for

prices on larger amounts................. .10

Tom Thumb, dwarf, mixed......................... .10

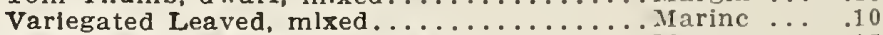

Lnnglfinra Aiba. sweet scented....................... 15

2.80

Mathiola Bicornis, Evening Scented Stock.........laturc ... . I5

Matricaria Capensls fi. pi., double white Fev-

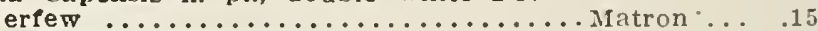

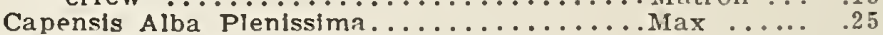

Eximia f. pl., Golden Ball...... i/ oz., 40c.. Martyr ... 1.40

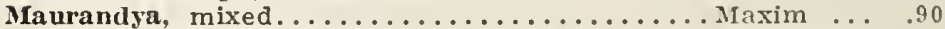

Purpurea Grandifiora..........1/8 oz., $25 \mathrm{c} .$. Mausoleum.

Barclayana Alba ..........1/8 oz., $25 \mathrm{c} . . \mathrm{Maw}$

Barclayana ...............1/8 nz.. 20c...1aul .... 1.25

Melothria Punctata, splendid annual climber .... Melo .... .80

\section{MIGNONETTE.}

Mignonette Grandiflora, large flowering, $100 \mathrm{lbs}$.

$\$ 35.00 ; 10$ lbs., $\$ 3.75 \ldots \ldots \ldots \ldots \ldots \ldots \ldots$ Microbe ... .10

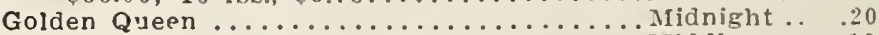

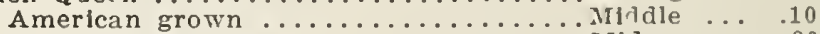

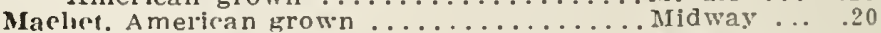

Vaughan's Selected Stock............................ 50

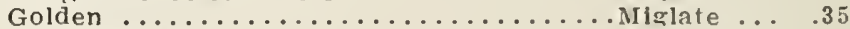

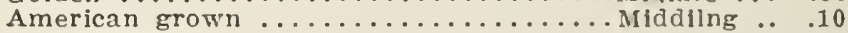

White Pearl ........................ 60 


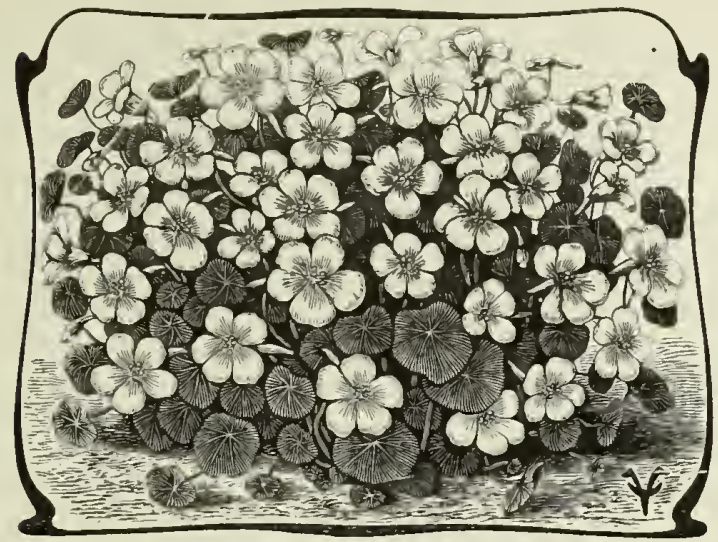

VAUGHAN'S NASTURTIUMS.

\section{Mignonette-Continued}

THL. CIPHER.

Oz.

LB.

Bismarcli, an Improved Machet........................ \$0.45

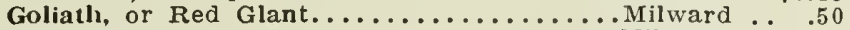

$\$ 5.00$

Mlles' Spiral ..................................... . . . . .

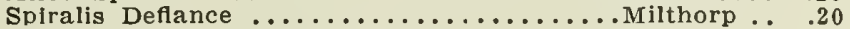

6.50

1.40

Gabriele, extra large and sweet................. .30

2.00

New York Market, for greenhouse,

3.50

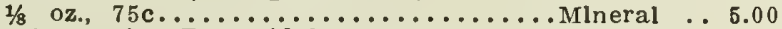

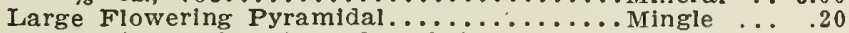

Giant White Spiral (Reseda Alba) ............. . . . .10

Many sorts mixed, 5 lbs., $\$ 2.40 ; 10$ lbs., $\$ 4.75$. Minify ... $\quad .10$

2.00

1.20

.50

Mimosa Pudica, Sensitive Plant ............ Minstrel ... .15

1.80

Tigrinus, slngle mixed........................60

Tigrinus fl. pl., Double Monkey Flower...... Misery ... 1.00

Large-Flowering, single (Queen's Prize).... Mister .... 1.20

Mina Lobata ............................... . ... 50

Sanguinea, one or our best annual climbers. . Miton ... $\quad .10$

6.00

.70

Mirablls, see Marvel of Peru.

Momordica Balsamlna, see Balsam Apple.

Monarda Didyma. . . 100 seeds, 25c; $1,000, \$ 1.50 \ldots$ Mist .....

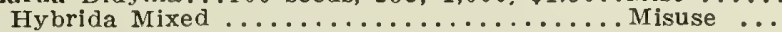

Moon Flower, see Ipomoea.

Morning Glory, see Convolvulus and Ipomoea.

Musa Ensete, per 100 seeds, $55 c ; 1,000, \$ 4.00 \ldots$ Musa ....

Myosotis, Forget-Me-Not, Alpestris, blue................

Alpestris. white ........................... .... 30

Indigo Blue, Vaughan's Royal Purple .. Myself .... .40

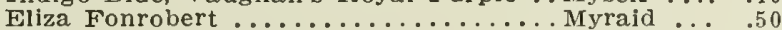

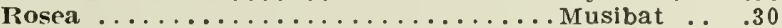

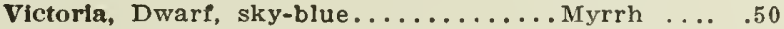

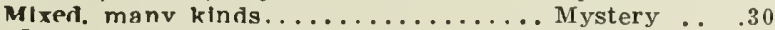

$3 . \cup 0$

3.50

4.50

6.00

5.50

Hybrida. Ruth Fischer, new, 1-32 oz.. \$1.50.. Musical ...

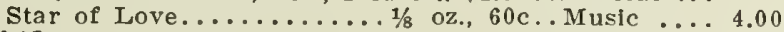

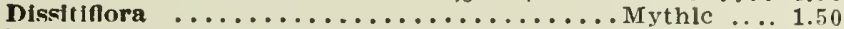

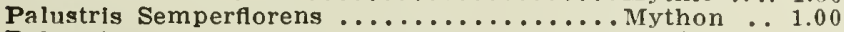

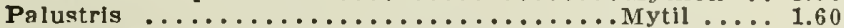

Ohlnngata, very fine, blue, blooms in 8 weeks. Mvtle ... .5n

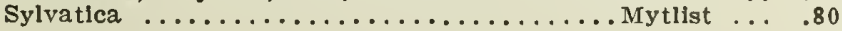




\section{DWARF NASTURTIUMS.}

We are headquarters on these and carry large sto cks.

TEL. CIPHER. $1 / 4$ LB.

LB. 10 LBS.

Aurora, chrome yellow, blotched crimson... Naples ... \$0.15 \$0.40\$3.50

Bronze ....................... Nardoo ... .15 $.40 \quad 3.50$

Beauty, scarlet, blotched canary.........Narcoma .. $.15 \quad .40 \quad 3.50$

Chameleon, mixed, true French strain......Nargil .... $\quad .15 \quad \mathbf{4 5} \quad \mathbf{4 . 2 5}$

Cloth of Gold, flowers scarlet, foliage yellow. Narrate $\ldots \ldots .15 \quad .45 \quad 4.25$

Cooclneum, dark scarlet.............. Narrow ... $.15 \quad .35 \quad 3.25$

Crystal Palace Gen, sulphur, spotted

maroon.................................. $\quad .15 \quad .35 \quad 3.25$

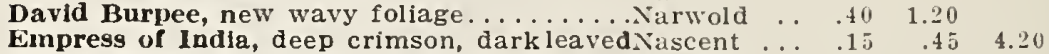

Golden IKing, rich golden color, true........Nassau .... $.15 \quad .45 \quad 4.20$

King of Tom Thumbs, deep scarlet, dark-

leaved................................... $\quad .15 \quad .45 \quad 4.20$

King Thcodore, rich red, dark bluish follage. Nasute $\ldots . .4 .15 \quad .40 \quad 3.80$

Ladyblrd, yellow, barred with crimson..... Nasy ..... $.15 \quad .40 \quad 3.75$

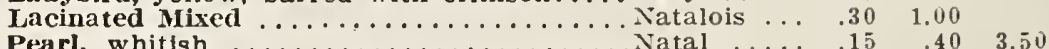

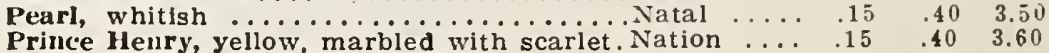

Queen of Ton Thumbs, crimson.........Nationate . .25 .90

Deep orange scarlet ................................. $40 \quad 1.30$

Mixed colors ....................................... . .20

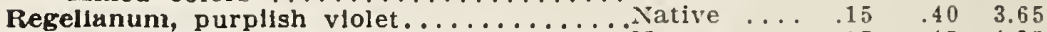

Rubs King, a pecullar blue-tinted red, true. Nature $\ldots \ldots 1_{15} \quad .45 \quad 4.25$

Spotted King. See Ladyblrd.

Vesuvius, a rich salmon rose, very fine. ... Naun $\ldots \ldots .15 \quad .45 \quad 4.20$

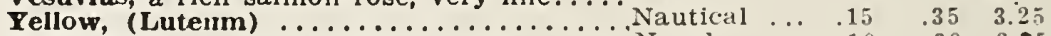

Dwarf Sorts, all colors mixed, $100 \mathrm{lbs}, \$ 20.00$. Naval .... $\quad .10 \quad .30 \quad 2.35$

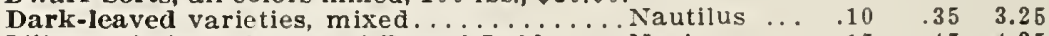

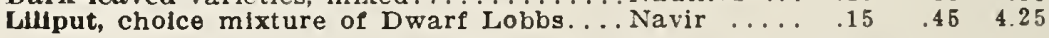

\section{“VAUGHAN'S SPECIAL MIXTURE"--Navy}

"Vaughan's Special Mixture" of Dwarf Nasturtiums wlll give a larger varlety of colors in even proportlons than any other Nasturtium mixtura for It is put up by ourselves from named surts, whlch have the richest and most varled combination of colors ever produced, showing odd colors hitherto unknown among flowers.

Per 1/4 Ib., 15c; Ib., 50c; 10 lbs., \$4.75; 100 lbs., \$45.00.

\section{TALL NASTURTIUMS.}

Butterty .................... Necktost . . .15 .4.

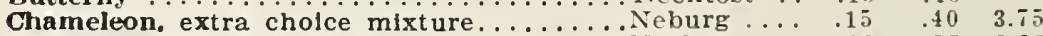

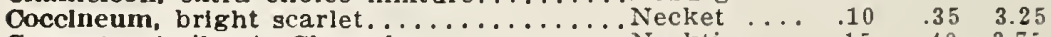

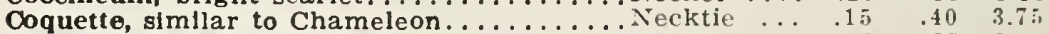

Dunnett's Orange (Sunlight), bright orange. Necre ..... $\quad .10 \quad .35 \quad 3.00$

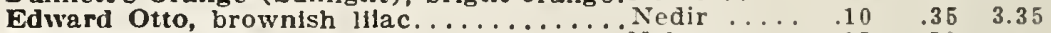

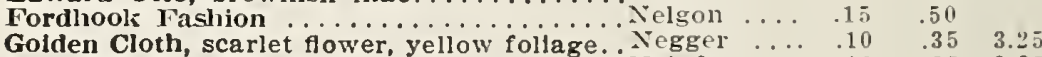

Helnemanni, chocolate color......................... $10 \quad .35 \quad 3.25$

Hemispherlcun, orange, very handsome..... Neiler .... $\quad .10 \quad .35 \quad 3.25$

Jupiter, Callfornia sort, yellow, large...... Neiling . . . . . $15 \quad 40 \quad 3.75$

KIng Theodore, rich, deep red, dark follage. Neinsook $\quad . \quad \begin{array}{lllll}.15 & 40 & 3.85\end{array}$

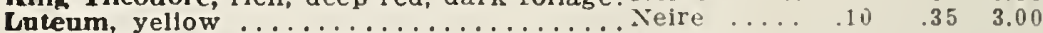

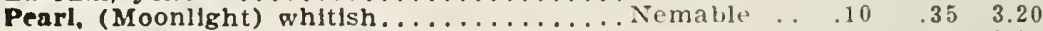

Prine Henry, light yellow, marbled scarlet. Nemeless .. $\quad \begin{array}{llll}10 & .35 & 3.30\end{array}$

Regcllanum, rich crimson, one of the best. Nemesake $\begin{array}{rrrr}.10 & .35 & 3.20\end{array}$

Scheuernianl, straw color, spotted.........Neming ... $.10 \quad .30 \quad 2.80$

“ Cocclnenm. scarlet striped.... Nency $\ldots . .10 \quad .35 \quad 3.20$

'Twilght, bright yellow marbled salmon rose. Nendogty ... $\quad .15 \quad .40$ 


\section{TALL NASTURTIUMS-Continued.}

Shtilingi, bright yellow, maroon blotches... Nendino .. \$0.10 \$0.35 \$3.20

Vesuvlus, salmon rose, dark-leaved, extra... Nendus ... $\quad .15 \quad .35 \quad 3.30$

Von Moltike, bluish rose................ Nenism ... $.10 \quad .35 \quad 3.25$

Tali sorts in finest mixture, 100 lbs., $\$ 18.00$. Netar $\ldots \ldots .10 \quad .25 \quad 2.00$

Madain Gunther's IIybrids, true......... Neuf ......15 $.40 \quad 3.75$

Variegated leaved mixed................ Nibated ... $.15 \quad .40 \quad 3.75$

VAUGHAN'S SIECIAL Mixture of Tall Nasturtiun-Tel. Cipher Nett. Includes the above sorts of Tall as well as the Lobb's Nasturtiums. the beautiful IIybrids of Madam Gunther, and the Canary Bird Creeper, and is the finest mixture ever offered. $1 / 41 b ., 15 c ; 1 b ., 50 c ; 10$ Ibs., \$4.75; 100 ibs., \$45.00.

\section{LOBB'S NASTUR'TIUM.}

Asa Gray, yellowish white............... Transmit $\$ \$ 0.10 \$ 0.35$

Biack Princo ....................... Trance ... .15 $\$ .50$

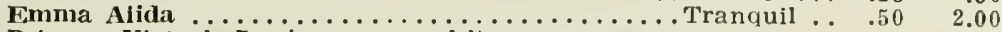

Princess Victoria Louise, cream white, orange-scar-

let biotches: calyx and spur orange-red...... Trawler ... .15

Spltnre, brilliant scarlet................ Trapper $\ldots . .15$

Raglna, salmon, extra fine............... Tropical . . 15

Giant of Battles, sulphur wlth red........... Travel ... .10

Brliliant, dark scarlet................... . . . . .

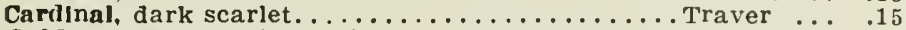

Golden Queen, golden yellow............. Trawling .. . I5

Lucifer, very dark scarlet.................. Tray .... .15

Napoleon III, golden yellow, spotted with brown..Trazer .... .15

Queen Wrihelmina .................... Tread .... .20

Queen Alexandm, flowers blood red..........Traverse.. .25

Queen of the Morning. flowers aurora colored Treiste ... .25

Queen Emma, flowers scarlet...........Treadest ... .15

Queen of Spain, flowers golden yellow....... Transform. .20

Queen type (var. leaved sorts) mixed...... Trawl .... .20

Fimbriatum, fringed flowers .............. Transfer .. .15

Klng of the Biacks, black-brown............. Treading .. .15

Flrefly, fiowers dark orange, spotted and flamed

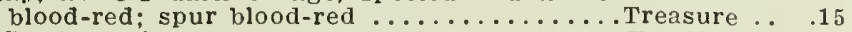

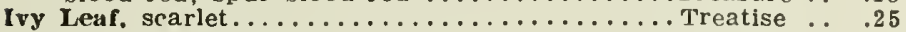

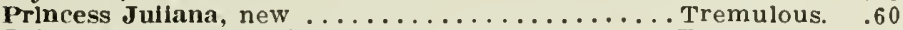

Primrose, ereamy-white with brown spots....... Tremor ... .15

Virchow, deep ruby rose.................. Tropico $\ldots . .15$

Iany Colors Mixed, $100 \mathrm{lbs}$., $\$ 22.00 ; 10$ lbs., $\$ 2.50$. Tropocl $\ldots . .10$

oz.

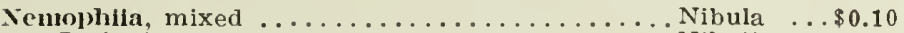

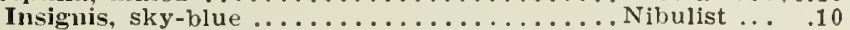

Nlcotiana Affinis, large, white, very tragrant. . . . Nickel . . . . 15

Aflinis Hybrids ....................... Nook .... .25

Sylvestrls, very beautiful, sweet, pure white.. Niedlic ... 10

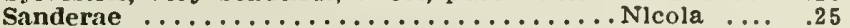

New Hybrids, mixed ............... Nibble ... . 25

Nlerembergia Gracllis, light blue, dwarf........ Nigard ... .40

Nigelia, mixed................ $10 \mathrm{lbs} ., \$ 3.75 \ldots$ Nigella ... .10

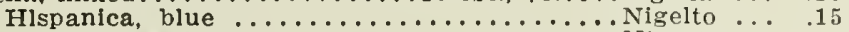
Alba, white .................................

Damascena, Miss Jekyll, double blue........ Nigastow .. .20

Nolann. mixed ............................... 10

Nycterinia Canensis, white, very sweet. 30 oz.. $10 \mathrm{c}$. Nyctea ... 50

Oenothera, Evening Primrose, annual varieties,

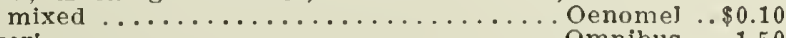

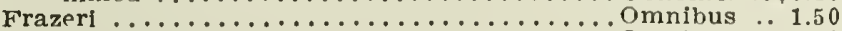

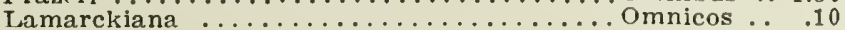

Macrocarpa ......................... 30 


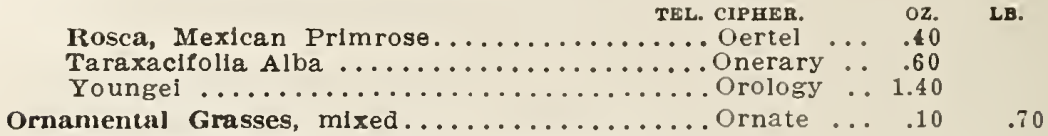

Oxalls Tropæololdes; dark yellow flowers, brown

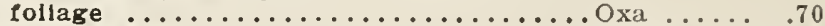

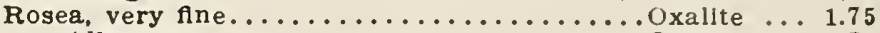

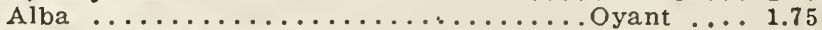

Valdiviana, yellow, sweet......................

\section{PANSIES. \\ GIANT-FLOWERED SORTS.}

We are having our Pansy seed grown by some of the best European Pansy specialists and are therefore in position to offer the best quallties at prices that are as low, and In many cases, lower than those of German wholesale seedsmen. We invite comparison.

TEL. CIPHER. OZ. LB.

Glant Adonis, light blue...............Pacage ..\$0.85 \$10.00

Andromeda, rose with lavender $1 / 8$ oz., 20c..Pacer $\ldots . .1 .2516 .00$

Atrosanguinea, dark red ......................... 1.00

Aureola, lower petals crimson, with dark

blotches; upper petals light yellow........ Pacific ... 3.00

Auricula Flowered (Bronze), fine shades.... Pacifier ... .75

Aurora, pure white....................... 0.75

Beaconsficld, purple violet, top petals blue... Padous ... .75

Black (King of the Blacks) ...................... . 80

Boulogne Giants, extra.............Pagjot ... 10.00

Bridesmaid, lovely apple-blossom...........Paginer .. 1.00

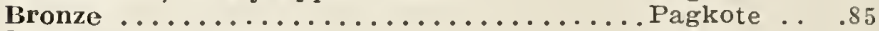

Bugnot, extra choice ................Pagode ... 1.25

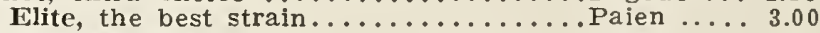

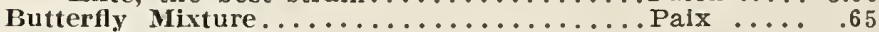

Cardinal, brilliant red ...................... 1.50

Cassier, 3 and 5 blotched, extra.............Paison ... 1.00

5 blotched yellow (Pres. McKinley).... Paitre ... 1.25

5 blotched white (Pres. Carnot)........Pair ..... 1.00

Hortensia-red ..................Palable. . 1.00

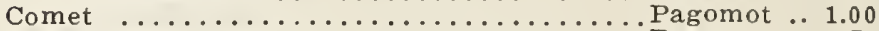

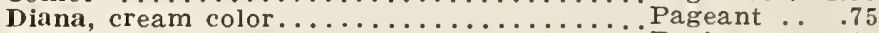

Eros, velvety brown, edged golden yellow...P. Paging ... 1.80

Emperor William, rich metallic blue.......Palais .... .75

Emperor Francis Joseph................Palmistry . 2.00

Ficry Faces, (Fire KIng), red with black

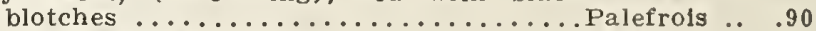

Freya, sllver-edged purple.......................... 80

Golden Queen, pure yellow........................ .9.

Indigo King, indigo blue..........................

Mad. Pcrret, lovely shades of rose and pink..Paleur ... .65

Masterpiece, new strain with fluted petals...Pallr ... . 1.00 Origlnator's stock ....................... $\$ .50$

Mauve Qucen, dellcate mauve, lower petals blotched carmine ................Palissya ... .80

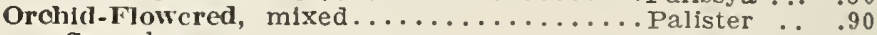

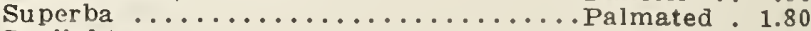

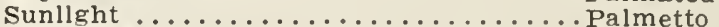

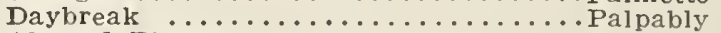

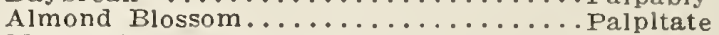


PANSY-Giant Flowered Sorts, Continued.

Tel. Cipher. Oz.

Lb.

Pretiosa, ground color rosy crimson, violet

blotches white margin.............Palmira . $\$ 1.00$

Prince Bismarck, light brown shades.......Palot..... .80

Prince Henry, darkest blue.............Palovate .. 1.0015 .00

Princess, a new type..................... 4.50

Purple, rich color....................... 85

Purple King ......................... 1.20

Psychc, violet bordered white, 1/8 oz., 25c... Palus .... 1.75

Rapliael, blue veins and violet blotches on

white ground ............................ 3.00

Rosy Morn, purplish crimson with clear edge.Paddle ... 2.00

Ruby-Red, rich shades of red............ Pantheon . .90

Siegfricd, brown shades, white margined....Pantheist , 2.00

Striped, a fine strain........................ .85

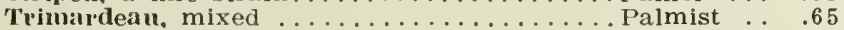

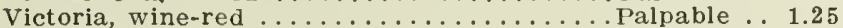

Violet Blue ........................... .80

Vulcano, dark red with large blotches....... Pampfly ... 1.60

White, with large purple eye............ Pamphlet . .80

Yellow, with large black eye............. Panache .. .75

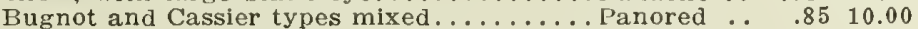

\section{VAUGHAN'S GIANT PANSY MIXTURE}

This mixture is specially made up by ourselves from all of the separate colors of the Giant Pansies and several special strains which cannot be had in any other way.

TEL. CIPHER. 1/4 OZ. OZ.

LB.

Vaughan's Cut Flower Mixture........... Pandect 4.00

Pancarte $\$ 2.00 \$ 6.50 \$ 25.00$

PANSY-Chicago Parks Bedding Varieties

Atrosanguinea, dark blood-red..........Panse . . . \$0.15

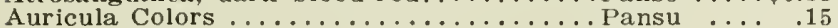

Azure-blue, velvety, dark..........................

Black, with Gold Bronze.............Panth .... .15

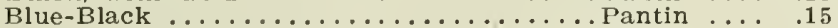

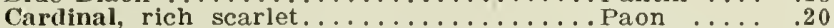

Coquette de Poissy, slate or mouse color... Papa .... .15

Dr. Faust, or King of the Blacks......... Papegal ... .15

Diana, crearn color..................Papeland .. .15

Emperor Frchlerick .................Papeterie ... .15

Emperor William, ultra-marine blue.....Papier .... .15

Fairy Qucen, sky-blue, silver border...... Papil ..... 15

Fire King, red, yellow margin........... Papilesse .. .15

Golden Gem, pure yellow. ............. Papilo .... .20

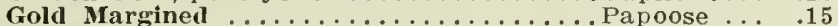

Light Blue, delicate shade.............Parade .... .15

Lord Beaconsfield, purple violet...........Paradox ... .15

Mahogany Colors ...................Paragon ... .15

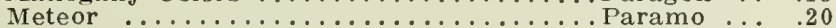

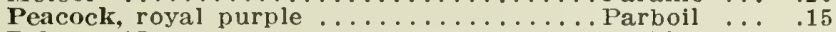

Pelargoniflora ........................... . 20

Prince Bismarck, bronze and light brown.. Parcel .... .15

Quadricolor (Pheasant's Eye), sky-blue,

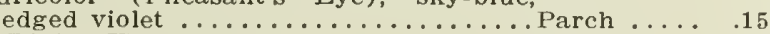

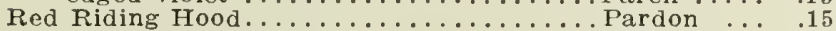

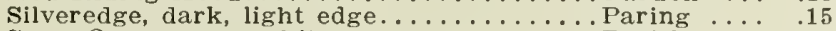

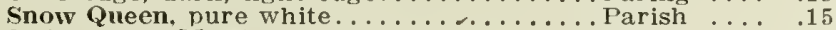

Striped and Mottled, extra choice..........Parity .... .15

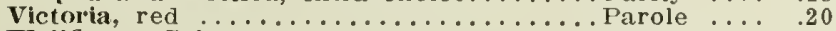

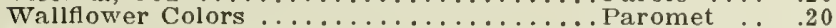

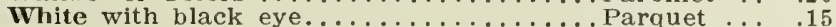

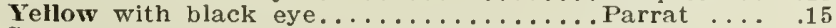

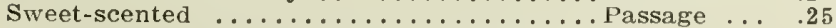

$\$ 0.60 \$ 7.00$

$.50 \quad 6.00$

$\begin{array}{ll}.50 & 6.00\end{array}$

.50

.60

$\begin{array}{ll}.75 & 10.00\end{array}$

$.60 \quad 7.00$

.556 .50

.60

.60

.50

.50

6.00

55

.658 .00

$.50 \quad 6.00$

$.50 \quad 6.00$

.556 .50

$.50 \quad 6.00$

.658 .00

$.50 \quad 5.50$

.658 .00

$\begin{array}{ll}.50 & 6.00\end{array}$

$\begin{array}{ll}.50 & 6.00\end{array}$

.556 .50

$.50 \quad 6.00$

$.50 \quad 5.50$

$\begin{array}{ll}.50 & 6.00\end{array}$

.657 .00

$\begin{array}{ll}.60 & 7.00\end{array}$

$.50 \quad 6.00$

$\begin{array}{ll}.50 & 5.00\end{array}$

CHICAgo PARKS BEDDING PANSIES-

Choice Mixed. All the preceding.... Panor ....\$0.45

PANSY, Good Mixed, many colors....... Pansage..... .30 


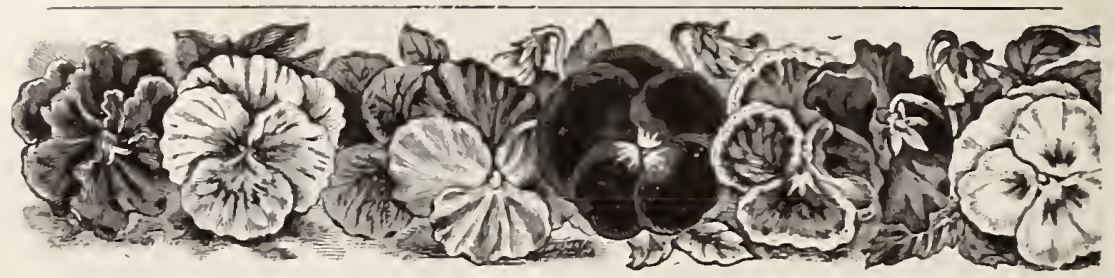

\section{VAUGHAN'S UP-TO-DATE : INTERNATIONAL-Pallia THE WORLD'S BEST PANSIES}

Vaughan's International is, we firmly belleve, the best Pansy mlrture In existence, because it is composed of the cream of 10 Pansy Specialists collections. It contains besides the fancy selections of these growers all the separate colors and strains in cultlvation, carefully prepared by ourselves, so we know just what it should produce.

This is one of the specialties which has establlshed the reputation of Vaughan's Seeds, and our customers can readily see why it is our most carnest endeavor to make Vaughan's International Pansy Mixture better than erer. Per $1 / 8$ oz., $\$ 1.00 ; 0 z ., \$ 7.00 ; 1 b ., \$ 90.00$.

\section{MPROVED GERMAN MITCRE PANSIES.-Panne.}

This is a mixture of three strains from different German Growers, and is made up mostly of separate colors, with sufficient percentage of white and yellow.......................... $\$ 1.00 \$ 12.00$

Papaver, see Poppy.

TEI. CIPEER.

oz.

LB.

Passiflora, Mammoth Passion-flower, blue.......Passing ...\$0.70

$\$ 8.00$

Incarnata .....................Passable . . 40

Gracllls ................................... 40

Pelargonlum, see Geranium.

Pennisetum Rueppelianum, Purple Feather Grass.Passion ... .20

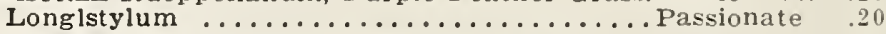

Pentstemon, large-flowering hybrids, mixed...... Peage ... $\quad .90$

Dlgitalls, white tinted vlolet.............Peach .... .20

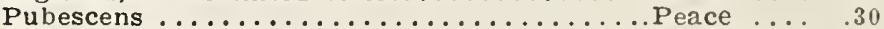

Pulchellus Hybridus.................Peaceable . .50

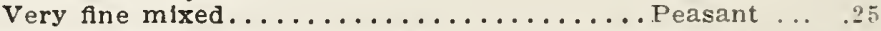

Pepper, Vaughan's Xmas (Chamaleon).1/8 oz., 15c.Pegasus ... 1.00

Peony, Double Chinese................. Peony ... . .30

Perilla Nankinensis....................Perllla ... .10

\section{PETUNIA HYBRIDA.}

l'etunia Hybricla, good mixed ....5 lbs., \$17.00 . Peerage .. .30 $3.5 n$ Hybrlda, finest mxd. (part from named sorts).. Peevish ... . 40 4.50) Alba, whlte .....................Pelagic ... 40 40.100

Carmen Sylva, (Baby Blue).................... .70

Blotched and Strlped (Inlmitable)........ Pellet .... .65

Snowball, Dwarf whlte................ Penance . . 90

General Dodds, blood-red...................... .40

Countess of Ellesmere, pink with white throat. Pencast .. .30

Venosa, llght red, velned...................... 35

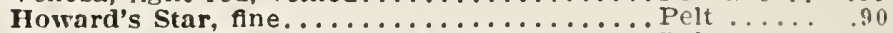

Hubrida Nana Comnacta. Rosy Morn........ Pelter .... .90

Nana Compacta, Gloria, pink............................. 2.40

nware Inlmitable. hlotched and strlned...... Pelting ... 60

Vaughan's Speclal Mixture Dwarf Petunias..... Peltert .... 2.00 


\section{PETUNIA-Large=Flowering Sorts.}

We call particular attention to our large-flowering strains of these, which are unsurpassed for size, shape and variety of coiors.

TEL. CIPHER. OZ.

Large- Flowering, finest mixed ...1-16 oz., \$1.75..Pencil . $\$ 24.00$

Large Flowering, good quality, mixed, 1/8 oz., 50c. Penciled . 3.00

Fringed, mixed, extra choice..1/8 oz., 4.00..Pendant ..

Giants of Califorula, true..1-16 oz., 1.75.. Pensive . 24.00

Triumph of the Giants, the most beautiful strain of large flowering single petunia. .

$\ldots \ldots \ldots \ldots \ldots \ldots \ldots$ oz. for $4.00 \ldots$ Pensively..

liurled Giauts, extra choice.1-16 oz., 2.50.. Pentact ...

I3alcony Queen, dark indigo blue with white

rays ............1-32 oz., \$1.00..Pentinkage

Pink ................1-32 oz., 1.00..Pentink ..

White ..............1-32 oz., 75c..Penture ..

Vaughan's Best mixture of large flowering sorts includes all the above and others..

................1-16 oz.,\$2.50..People ...

\section{Double=Flowering Sorts.}

Double Large-Flowering, extra choice mixed, 1,000 seeds, $\$ 1.00 ; 10.000$ seeds, $\$ 9.00 \ldots$

1-64 oz., $\$ 2.75 ; 1-32$ oz., $\$ 5.00 \ldots \ldots \ldots$. Perfectlon

Double Large-Flowering, frlnged, best mixed, 1,000 seeds, $\$ 1.00 ; 10,000$ seeds, $\$ 9.00 \ldots$

......1-32 oz., $\$ 4.50 ; 1-64$ oz., $\$ 2.50 \ldots$ Period

Double Large-Flowering, good mixed........

.......... 1/8 oz., $\$ 5.00 ; 1-32$ oz., $\$ 1.50$..Perios

Lady of the Lake, double-fringed, pure white,

1,000 seeds, $\$ 1.20 ; 1-64 \mathrm{oz} ., \$ 2.75 \ldots \ldots$ Permit

Double Fringed Perfection, 10,000 seeds,

$\$ 9.00 ; 1,000$ seeds, $\$ 1.00 ; 1-64$ oz., $\$ 2.50$. Perquisite

Double Fringed Pink.....1,000 seeds, $\$ 1.30$.. Perfecto

Vaughan's Special Mixture. Double, extra

choice, 1,000 seeds, $\$ 1.20 .1-64$ oz., $\$ 3.50$. Perqueo

oz. LB.

Phacella Tenacetifolla, blue......................... $\$ 0.10 \quad \$ 0.40$

Plieasant's Eye Pink, see Dianthus Plumarius.

\section{PHLOX DRUMMONDI GRANDIFLORA.}

White ............... Phase.

Pink ................. Pheasant.

Bcarlet ............... . Phenic.

Stellata Splendens......... Phire.

Black Brown. . . . . . . . . Phiring.

Rnspa Striata............ Phlrka.

Brilliant Rose dark eye Pew.

Carnea (Flesin)......... Pheaton.
Alba Oculata.......... Phoca.

Coerulea Striata......... Phocold.

Scarlet Striped..........Phœbo.

Yellow ................. Phonade.

Kermesina Splendens..... Phonai. Orbieularis Violacea .... Phantasmorbicularis Atrorosea..... Phalanx.

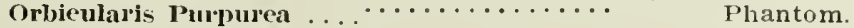

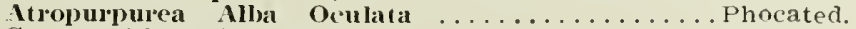
Crean witl lied Fye.... $\ldots \ldots \ldots \ldots \ldots \ldots \ldots$ phocare.

Crown Prince, fierv scarlet, oz., $\$ 1.00 \ldots \ldots \ldots \ldots \ldots$. . . . . . . . . .

Rosea Aurea Stellata (Gold Star) oz., $\$ 1.00 \ldots \ldots$. . . Wharisee.

Fach of above, except those priced, pel oz., 50c; 1h., $\$ 6.00$

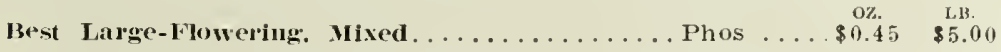

Imported Collection, 6 varieties, ea., $15 \mathrm{c}$, net.Pholobo ...

Imported Collection, 12 varietles, ea., 25 c, net.Pholobox॰. . 


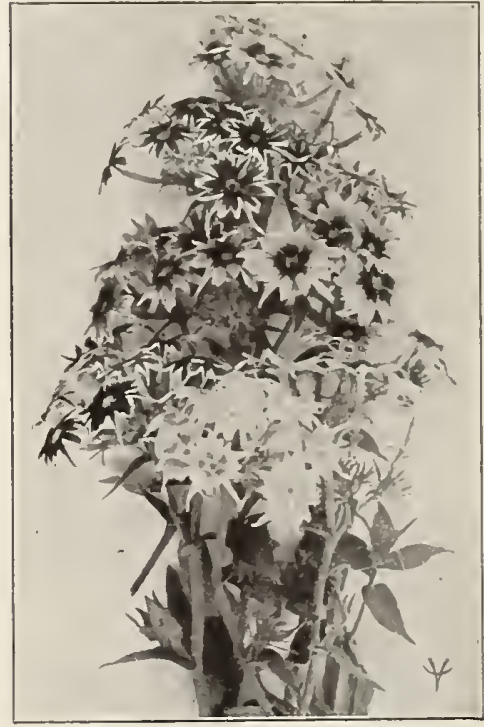

PHLOX Star of Quedllnburg

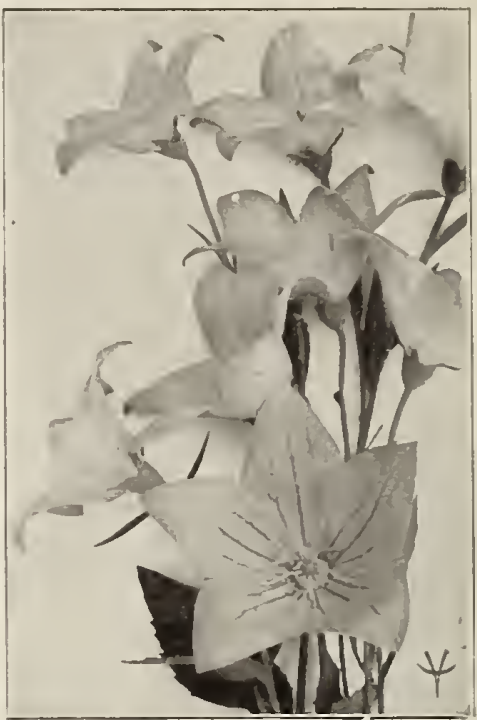

PLATYCODON

\section{PHLOX, Continued}

Phlox Drummondi, fine mixed............. Phosphor $\$ 0.40 \$ 4.50$

Double Yellow, Isabellina semi-plena...... Phrase ... 1.00

White, Alba semi-plena........................ .90 12.00

Red, Atropurpurea semi-plena......... Pholopur . 1.00

Mixed, the above and others..........Phro .... $.85 \quad 10.00$

Star of Quedlinburg (Star Phlox), mixed.... Phron ... .50 6.00

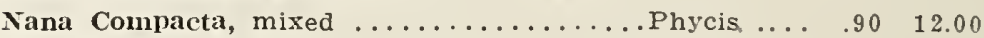

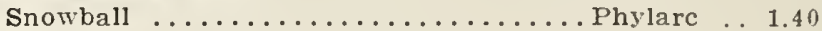

Fireball .............................. 1.25

Large-Flowering Cecily, mixed........ Phial ....

Hortensia-Flowered, extra choice, mixed.... Physac ... .60

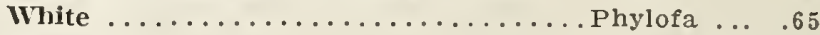

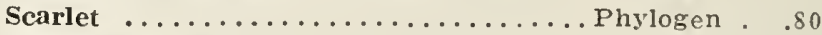

Brilliant Rose ................. Phyloka .. . .80

Salmon Rose...................Phyloked . 1.00

Vaughan's Speclal Mixture, includes all the

varieties listed above and others....... Physiol ... .75

Decussata, perennial varieties, mixed...... Physique . . S5

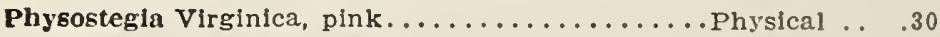

Virginica Alba, white.......................... .70

Pinks, Chinese and others, see Dianthus. 
TEL. CIPHER.

oz.

Platycodon Grandiflora, blue..................... $\$ 0.85$

Grandiflora Alba, white.............. Plateau ... 1.00

Double Blue .............................. 1.80

White ................. Plaid .... 2.00

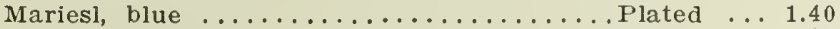

fl, albo, white......................... .80

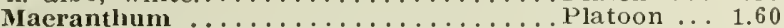

Polemonimn Coeruleum Grandiflorum, blue......Platron ... .20

Coeruleum Grandif. Album, white......Platrs ... . .10

Polyanthus, mixed (Primula Veris) ............ Polyad ... .75

\section{POPPY-Papaver.}

Ameriean Nag, white with scarlet...........Ponkate .. .10

Alpinum Laciniatum ................... Pomado ... 2.40

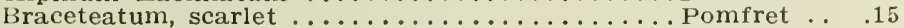

Danebrog, with large white spots..................... 10

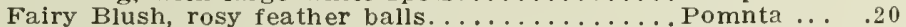

Golden Gate .......................Pomnzar .. .15

Glaneum, Tulip Poppy, deep scarlet flowers..... Pompous . 20

Hansa, single white edged carmine..................... .10

King Edward, deep scarlet......................... .20

Laevigatum, Persian or Firedragon......................... 10

Mephisto, deep scarlet with blackish violet spots. Pomras ... $\quad .10$

Murselli f. pl., Mikado, white; crimson striped.. Pomsart .. .10

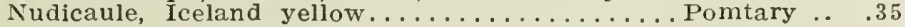

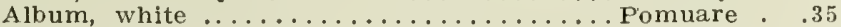

Coccineum (aurantiacum) scarlet.......... Pomvate .. .60

Striatum striped .................. Pomway .. .60

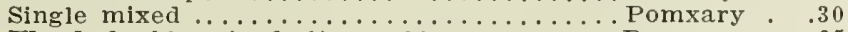

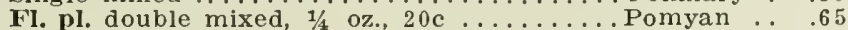

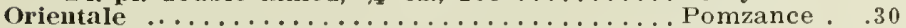

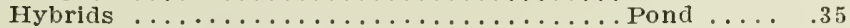

Princess Victoria Iouise. . . . . . . . . . Porgany $\quad \therefore 1.00$

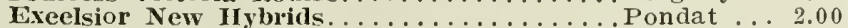

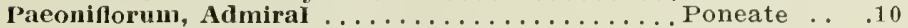

FI. pl., paconyfld, double, white...........Ponfer ... .10

“. " " "

" " " $"$ pink ............

" " $"$ " $"$ scarlet ...........

mixed $\ldots \ldots \ldots \ldots$

$\ldots \ldots \ldots \ldots \ldots \ldots \ldots \ldots$ ibs., $\$ 3.0 \ldots$. Ponjater

.10

.60

1.40

.40

2.00

1.80

2.40

1.40

2.00

1.30

.40

.50

3.80

3.80

3.00

8.00

3.50

4.00

.50

1.00

1.00

1.00

1.00

.40

Pavonium, Peacoek, brilliant-scarlet with glossy

black ring ............................... .20

Rhoeas single English scarlet........................ .20

F1, pl, double Freneh mixed .............. Ponnay ... . 10

Shirley ............................... 10

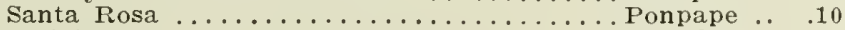

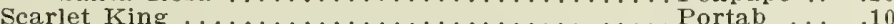

Somniferum, single, Black Prince....................... .10

$$
\text { "t "The Bride, white............... Ponryan ... } 10
$$

fl. pl., double Carnation-Flowered,

mixed.........10 lbs., $\$ 3.50$..Ponszat .. $.10 \quad .40$

“ nanum f. pl., Caidinale, scarlet.... Pontal ... . .

$.10 \quad .60$

Tmbrosum, bright vermillion with black spots... Ponvatie ... 10

Fl. pI., producing about 50 to $60 \%$ semi-

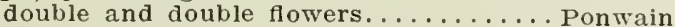

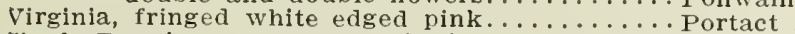

Single Poppies, many sorts, mixed. 10 ibs., $\$ 3.00 \ldots$ Ponxent

Double Poppies, many sorts and colors, mixed...Ponzahn

Vaughan's Special Mixture, Single Poppies...... Ponzater

" " $"$ Poppy Mixture, Includes all

the double and single (annual varieties). Ponzaster
$.10 \quad .60$

$.10 \quad .85$

.70

.10

.10

.10

.15

.15

2.00

2.40

.70

1.00

1.40

1.00

1.00

.60

80

.15
.85

.35

.40

1.60

1.00

1.50 


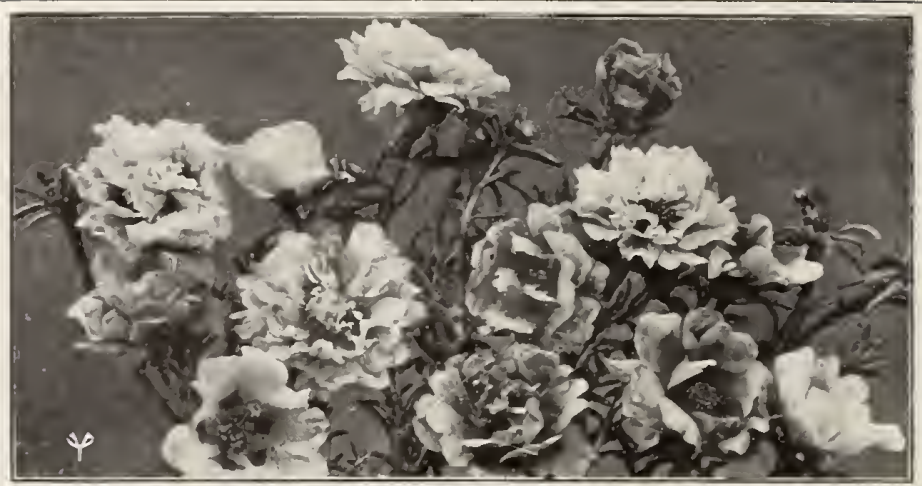

PURTULACA

Single Large-Flowering.

TEL. CIPHER. OZ. IB.

White ......Pottage. $\$ 0.20 \$ 2.40$

Pirk .......Portal... .20 2.40

Yellow ......Potash... .20 2.40

Parana, New Giant flowered............... Potancing . .50

All colol's mixerl. . ...........5 lbs., \$8.50..Potion ... .15

TEL. CIPHER.

oz. LB. Scarlet ...... Potance. . $\$ 0.20 \$ 2.40$ Striped .......Potanced. $20 \quad 2.40$

Imported collection, 8 varieties..each, $20 \mathrm{c}$, net. . Portapo..

\section{Double Large Flowering.}

Oz.

White..per 1/8 oz., 30c. Pottage $\$ 2.00$ Pink...per 1/8 0z., 30c Pouch...\$2.00 Yellow.per 1/8 oz., 30c. Potter . 2.00 Scarlet.per $1 / 8$ oz., $30 \mathrm{c}$ Poultry.. 2.00

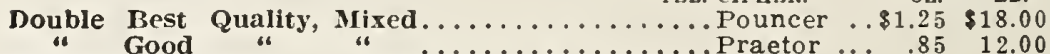
TEL. CIPHER.
OZ. LB.

Imported collection, 6 varieties, double, each $25 \mathrm{c}$. Porthole ..

Potentilla. Single mixed................... .30 Double mixed ...................................... 1.60

Formosa, light red.......................... 40

\section{PRIMULA CHINENSIS FIMBRIATA Large-Flowering Fringed Sorts-ENGLISH GROWN.}

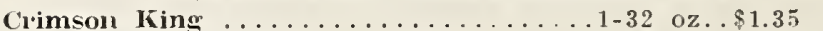

Queen Alexandra, giant white........

Orange king ................... 1-32 oz.. 1.50

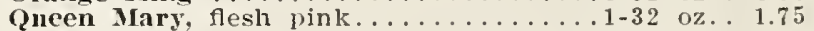

Giant Pink ........................ oz . 1.75

King George, crimson........... 1-32 oz.. 1.75

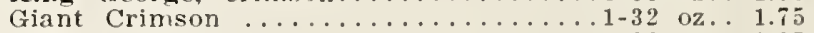

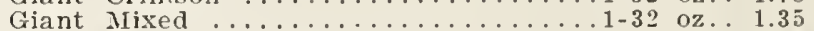

Delicata .................. 1-32 oz.. 1.20

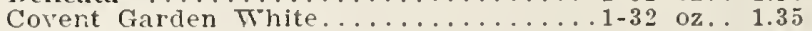

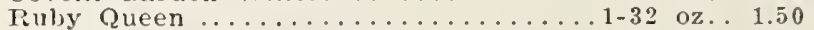

True Blue ................ 1-32 oz, 1.75

Chiswick Red, Improved........... 1-32 oz.. 1.35

Giant Salmon ............................ 1.75

Vanghan's International Primula Mixture. 1-16 0\%.. \$3.25;

$1 / 8$ oz., $\$ 6.25$; per oz., $\$ 45.00 \ldots \ldots \ldots$.

Single Chinese Primrose, mixed colors, is oz. $\$ 1.00 ;$ Oz., $\$ 6.00$.

PIRIIUIA STEIA.IT.I

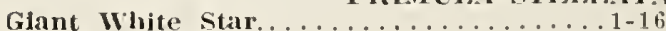

Blue Star Improved .............. 1-16

Crimson Star ...............1-16

Salnon Star ...............1-16
Prevent.

Prevented.

Primal.

Preventry.

Presta.

Prevention.

Preventist.

Prevensan.

Prenal.

Prey.

Prick.

Pretension.

Preterit.

Principal.

Prison.

. Primary.

oz...\$2.50 Primlst.

oz... 2.50 Primfay.

oz... 2.50 Primkot.

o\%... 2.50 Primlam. 


\section{PRIMULA-Continued. Various Sorts.}

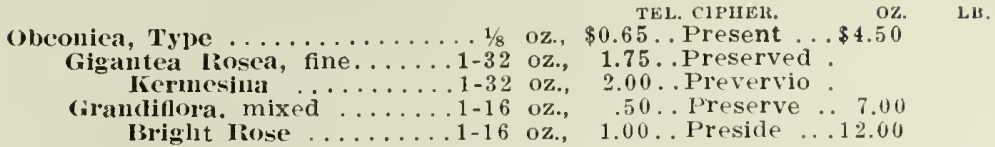

Besides the above strains, we can supply "Special Fiorists" stock, grown by the most celebrated $\mathrm{P}$. Obconica specialist. We will quote this strain on application.

Iorbesi, Baby Primrose........1/8 $0 \% ., 50 \mathrm{c} \ldots$ President . 3.50 Japoniea, mixed ............1/8 oz., 30c.. Presume .. 2.00

Kewensis...............1-16 oz., \$1.25. Previous...

Malacoides, Imp. Baby Primrose...1/8 oz., $\$ 1.00 \ldots$ Prevision

Sieboldi, large flowered, new varieties.1-32 oz., 1.00.Pressant

Veris Polyanthus (Cowslip), mixed........... Press ... . .75

New Giant Flowered, choice... 1/8 oz., $45 \mathrm{c}$. Pretestine .3 .00

Lutea Gigantea .................... Presumist . 4.00

Vulgaris, the true yellow Primrose.......... Presser ... 1.20

Pueraria Thunbergiana, Kudau Vine.........Puerto ... .40

Pyrethrum Parthenifolium Aureum, Gold. Feather.Prestine .. .15

Parthenlfolium A ureum Selaginoldes........Pyral .... . 30

Roseum Hybridum, single mixed......... Pyram ... .90

Grandiflorum, Giant, mixed............Pyrax ... 1.25

Double mixed, choice.... 1/8 oz., $\$ 1.10 \ldots$ Pyrite ... 8.00

New Hybrids . . . . . . . . . . . . Pyx .... 5.00

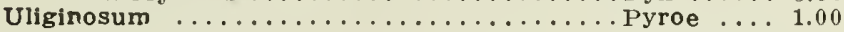

Relımanuia Angulata .........1/8 oz., 35c.. Repentant.

Rheum Palmatum Tanguticum..............Rhombold . .15

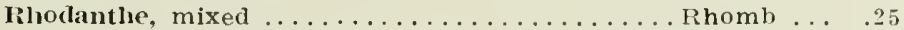

Rieinus Zauzibariensis, mixed...10 lbs., $\$ 6.50 \ldots$ Riches ... .10

Zanzibariensis Enormis ............... Riddance . .10

Borboniensis Arboreus .............. Ridpath .. .10

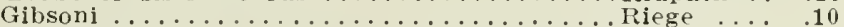

Sanguineus ............. 10 ibs, $\$ 2.70$. Riffler $\ldots .05$

Cambodgensis, very dark......5 lbs., \$2.50. Rifle ..... 10

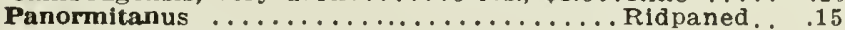

Mixed, many kinds.........10 lbs., $\$ 2.50 \ldots$ Rigid .... .05

Romneya Coulteri, white, large........... Roll .... .75

Rosa Polyantha Nana, Baby Rose, clean seed... Rosetta ... 2.50

\section{"VAUGHAN'S SPECIAL MIXTURES"}

Means the Best in Existence.

All of "Vaughan's Speeial Mixtures" are made up by ourselves from separate colors and specially fine strains, combined in desirable proportions. We spare no trouble or expense to secure for them the newest and choicest sorts, the widest possible range of shade and color. Vaughan's Speeial Mixtures" are full of life. We pride ourselves upon them, and our customers may depend upon it that any mixture listed 


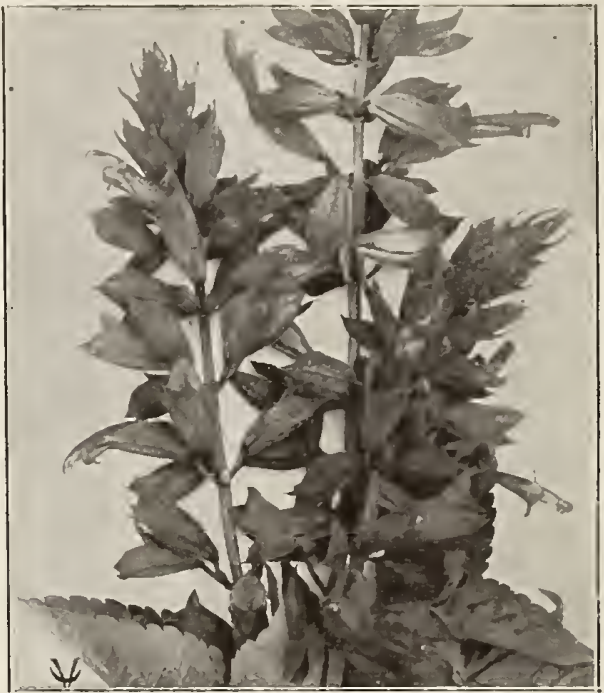

SALVIA SPLENDENS Clara Bedman

TEL. CIPHER.

OZ.

$\$ 0.15$

Bicolor Superba Semi-plena............. Rudbock .. .50

Fulgida, yellow, very free............... Rude .... .40

Nitlda, Autumn Glory.......1-16 oz., $80 \mathrm{c} .$. Rufflan ..

Golden Yellow, Large Flowering..........Rudeable . 1.25

Purpurea (Echlnacea) ..................Rueful ... 1.40

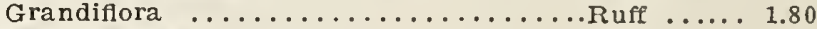

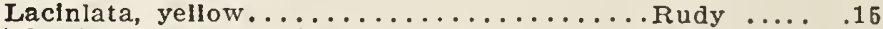

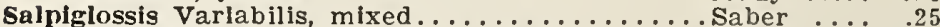

Large-flowering, extra cholce mlxed.........Sad ..... .40

Fimperor, mixed, very fine.......................... 75

Crimson ............................. 2.00

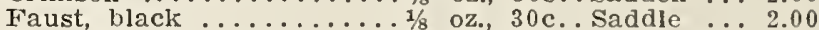

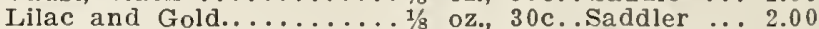

Pink ............................. ozaddlery .. 2.00

Purple Violet and Gold....1/8 oz., 30c.. Sadducee . 2.00

Purple Velvety Violet......1/8 oz., 30c..Sadly ... 2.00

Golden Yellow ................... 2.00

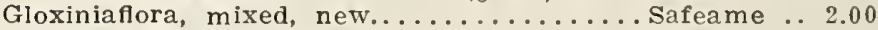

Vaughan's Special Mixture ............................... 1.60

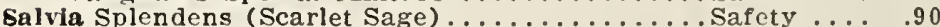

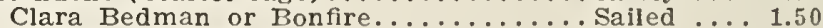

Drooping Spikes, very fine................... 1.50

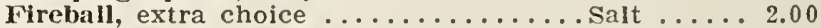

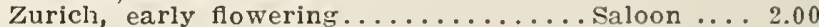

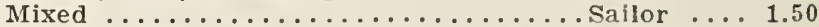

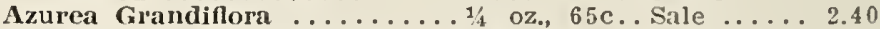

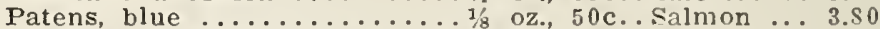

Coccinea, scarlet, annual flowering sage... Salvable .. .20

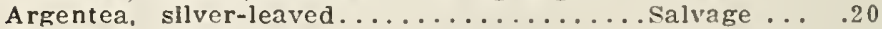

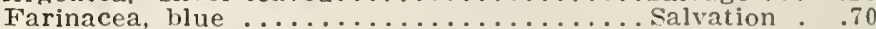

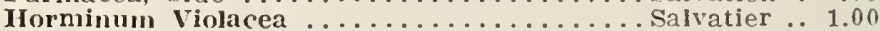

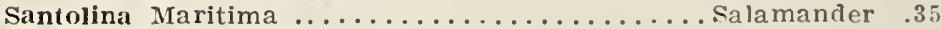

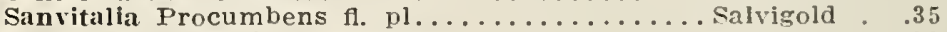

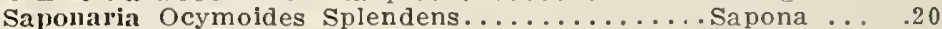

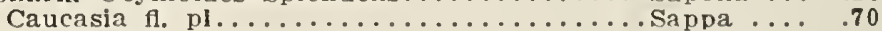

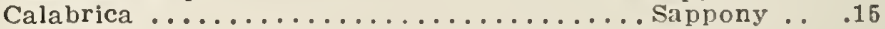

Vaccarla ...................................... 15
LB.

$\$ 1.80$

3.00

4.50

10.00

12.00

22.00

20.00

30.00

30.00

2.00

2.00 
TEL. CIPHER.

oz.

LB.

Scabiosa, Double Dwarf, mixed............................... . $\$ 0.15$

$\$ 1.80$

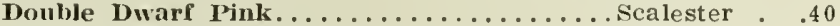

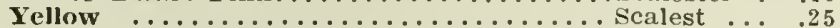

Lalde Flowering, Double Tall, mixed......... Scallop ... . 25

Atropurpurea Major, Tall, mixed...................... .15

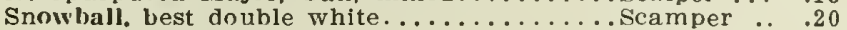

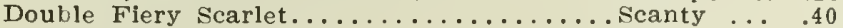

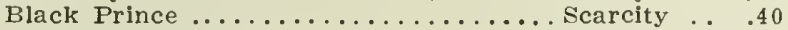

Mauve (Azure Fairy) ...................... . .50

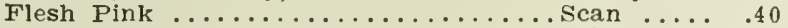

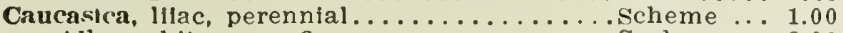

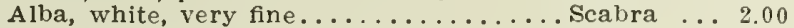

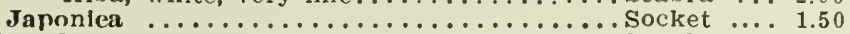

Schizanthus, (Butterfly Flower) mixed......... Scoul .... .10

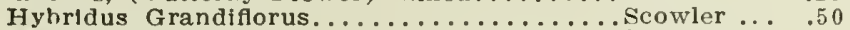

3.00

1.25

2.00

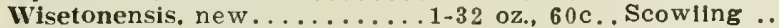
Bridal Veil.............................. 10cowled ..

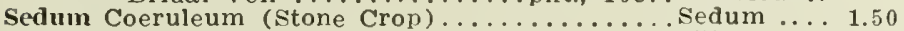

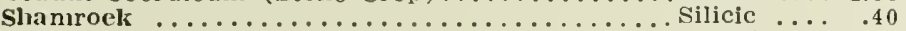

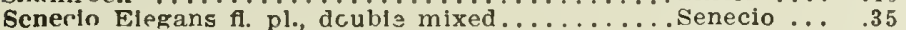

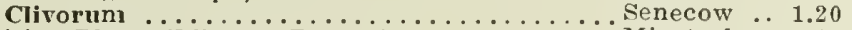

Sensitive Plant (Mimosa Pudica) .............. Minstrel . . . . . . . . . . . .

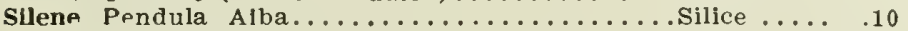

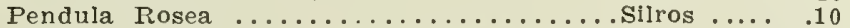

Orientalis, perennial, beautiful dark rose... Siek ..... 10

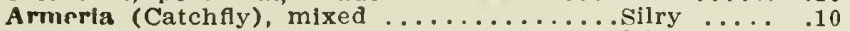

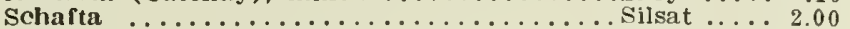

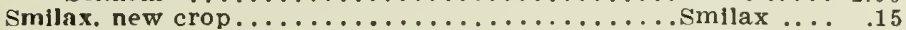

Snapiragon. see Antirrhinum.

Solanum Capsicastrum Nanum, Jerusalem Cherry. Sorrow ... .20

Capsieastrum Melvini ...................................... 1.00

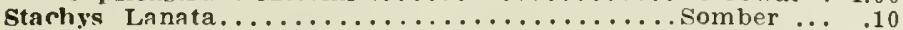

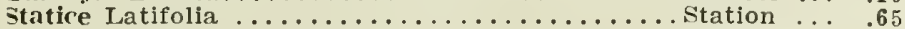

Sinuata, blue, fine cut flower.................................. 10

Suworowii (Russian) ............................................. .40

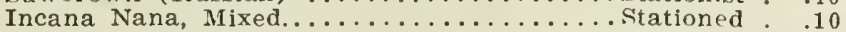

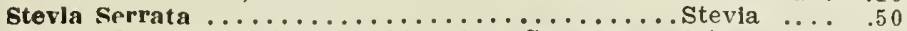

Lindleyana, a clearer white than Serrata.... Stiffe .... .60

\section{STOCKS.}

\section{Large-Flowering Double Divart German Ten Weeks.}

Our Stock Seed is from one of the best European Growers and is unexcelled, it is fust the quality florists want.

Crimson TEL. CIPHER.

Blood-Red ................. Stocky.

Brilltant Rose............... Stolcal.

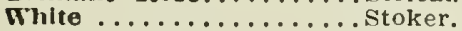

Canary Yellow............ Stolzcup.

Dark Blue................. Stooping.

Lirlit Blue ......... . . . Stoop.

Flesh .................. Stooped.

Each of above, $1 / 8$ oz., $25 \mathrm{c} ; 1 / 4$ oz., $40 \mathrm{c}$; Oz., $\$ 1.50$; Ib., $\$ 20.00$.

Extra Choice Mixed, all col-

ors, oz., \$1.25; lb., \$16.00 . Stoppage.

Good Mixed, oz., 50c; $\$ 6.00 \ldots$ Storage. Imirrted Collection, Large-

Flowering Ten Weeks

Stocks of 12 varietles, each, $30 \mathrm{c}$ net............. Straight.

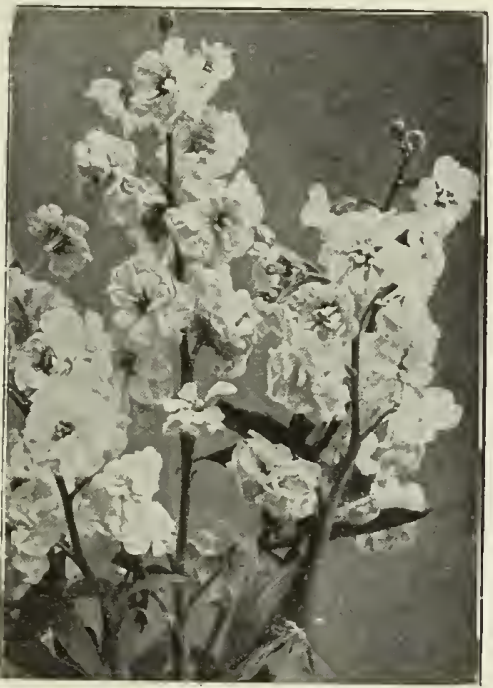

sTuCKs Beauty of Nice

5.00

4.00

1.80

.65

65

1.80

2.00

8.00

1.40 


\section{STOCKS-Continued.}

Imported Collectlon, Large-Flowering Ten Weeks

TEL. CIPHER.

oz.

L.

Stncks of 6 varletles.......edch, $16 \mathrm{c}$, net..strain ....

Glant Perfection, light blue.............. Stockful .\$1.50

Flesh color ................................ 1.60

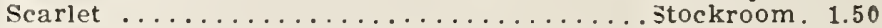

Pink ....................... Stockwork. 1.60

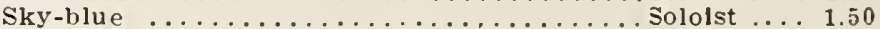

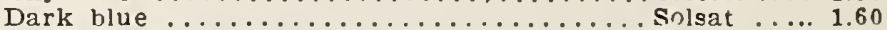

White (Princess Alice)......................... 1.50

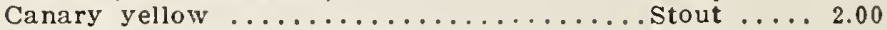

Mixed (Cut and Come again).................... 1.50

Imported Collection of 6 colors, $30 \mathrm{c}$ net..... Stocking.

Prince Bismarck, white............... Stow. ... 2.00

Lavender ................................ 2.20

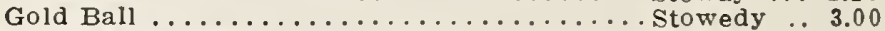

Beauty of Nice, plnk.................

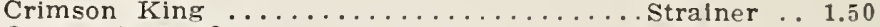

Queen Alexandra ................................ 1.40

Monte Carlo (Creole), canary yellow....... Stockton ... 2.00

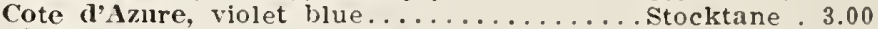

Abnndance, delicate rosy lilac........... Stockum .. 3.00

Mont Blanc ....................... Stocklum . 1.50

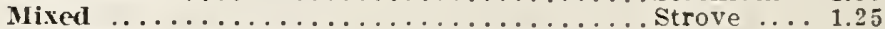

White Column, fine white........ oz., $40 \mathrm{c}$. . Stouten ... 2.50

Crimson Column ............. 1/8 oz., 50c.. Stolc .... 3.00

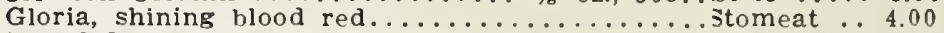

Snowflake, early white, forcing...1/8 oz., $\$ 1.00 \ldots$ Straggle .. 7.00

Vaughan's Fire Flame............1/8 oz., 45c.. Strive ... 3.20

Emperor or Winter, mixed.......1/8 oz., 45c.. Stroy .... 3.00

White ..............1/8 oz., 60c..stroying .. 3.50

Rose ................ oz., 60c..Stoniness . 3.50

Crimson .............. 1/8 oz., 60c..Stood .... 3.50

Empress Elizabeth ........1/8 oz., 60c.. Stoodine .. 3.50

Large Flowering White Lady. 1/8 oz., 80c. Stoodist .. 5.00

Vauglan's Special Mixture........................ 3.00

Stokesia Cyanea, Stokes' Aster..................... . 75

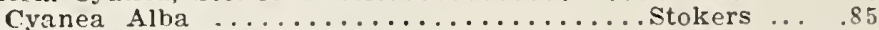

$\$ 22.00$

22.00

20.00

22.00

20.00

22.00

18.00

Streptoearpus. Largest Hybrids...... pkt., 12c.. Strepts ...

Swainsonia Grandiflora Alba................... 2.00

\section{SUNFLOWER.}

Sunflower, (Miniature Type),Cucumerifolius....summons . .10

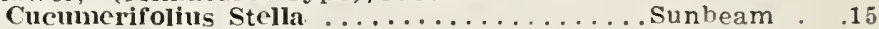

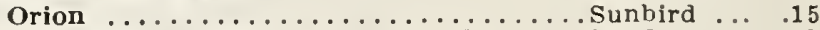

Purpureus, Miniature Red Sunflower ..Sunnora .. .50

Doulle IIybrids ................... Sunder ... .20

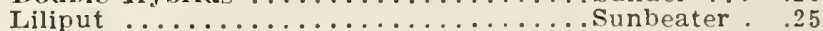

Vaughan's Special Mixed .................... .20

Annus Pallidus pl. Primrose Queen.................. .15

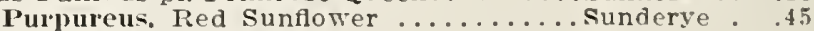

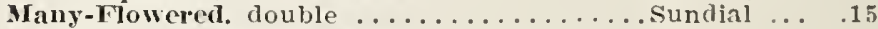

A revrophyllus (Sllver-leaved) ...................... 10

Californicus 1. pl.. double California...................... 10

1.20

9.00

10.00

Globnsus Fistulosus fl. pl., fine double........ Sunshine

Uniflorus Giganteus ............................ 10

Doulble Dwarf. (Nanus $\mathrm{n}$. pl.)................... 10

Double Chrysanthenum-Fowered ..........superba .. .10

Double, many kinds mixed ........................ 10

Vaughan's Special Mixture, all the above..... Supple .... .20 


\section{VAUGHAN'S SWEET PEAS}

Our Sweet Peas a re carefully grown by experienced men from stock seed in many cascs supplied by us, and better seed cannot be liad in this eountry than is supplied by Vaughan's Seed Store.

\section{SPENCER VARIETIES}

Afterglow. Bright violet standards shaded rosy

Tel. Cipher

Lb. amethyst, wings paler blue; waved................... $\$ 6.00$

America. White ground, heavily striped and

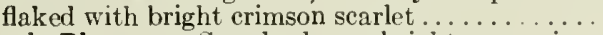

Apple Blossom. Standard very bright rose; wings

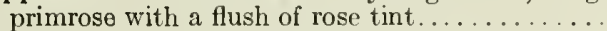

Asta Ohn. A soft pinkish lavender self. Flowers very large and beautifully waved..

Scripture

Aurora. Hlowers white, mottled and flaked with bright orange pink.

S'eil

Blanche Ferry. Rose standard and wings white, suffused and tinted with light pink.

Captain of the Blues. Pure purple, the margin of the petals is marbled. Very large and distinct

Clara Curtis. A beautifully waved cream of good substance, usually has four blossoms on a stem

Seiminal.

160

Countess Spencer. The original giant flowered Spencer, with finest waved standard and wings clear pink, deepening toward the edge.

Selation...

Senatorial.

160

Dainty. White ground, with beautiful picotee edge of rose pink, splendid form ................

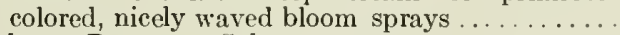

Edrom Beauty. Salmon orange. .

Elfrida Pearson. Large pale pink flower, the buds and young flowers having a distinct tint of buff

Ethel Roosevelt. Cream ground, striped crimson

Evelyn Hemus. Cream, with a pieotee edging of

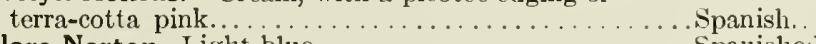

Flora Norton. Light blue.

Siegtar.

Sign.

Florence Morse. Standard richly flushed with a beautiful distinct shade of pink; wings soft blush pink..Spearade...... 150

Florence Nightingale. A bluish lavender........... Spank....... 240

George Herbert. Rose crimson, suffused with magenta and showing veins in wings ........... Specialty .... 160

Helen Lewis. Blooms large orange salmon with pink, especially bright orange standard............

Hercules. A pale rosy pink flower of giant size.

Snash.

Soda

Soap

600

Soberty.

Sobriety

Spaniard.

175

King Edward VII. The best pure red Spencer, very large, with espeeially immense wings.

King White. Gigantic flowers of pure white.

Lady Knox. Deep cream, beautifully edged

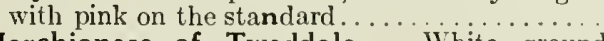

Marchioness of Tweddale. White ground

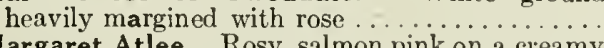

Margaret Atlee. Rosy, salmon pink on a creamy buff ground. Immense wavy standard, inclined to double. Large wavy wings........ Oz. $\$ 1.00$

Margaret Madison. A beautiful self colored azure blue. A lovely sharle

Spin

Spinal

Sportive

180

Sportful

600

Spindle

600

Spine.

400

Spinarl

Spinel 


\section{SPENCER VARIETIES-Continued}

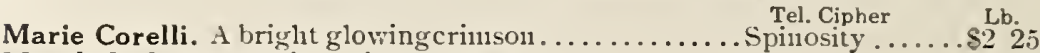

Maud Holmes. Rich crimson Spencer................ Spiracle........ i 80

Mrs. A. Ireland. Standard bright rose pink,

witli creanly base, wings blush $\ldots \ldots \ldots \ldots \ldots \ldots$. Spirit......... 175

Mrs. Cuthbertson. Standard rose-pink, wings

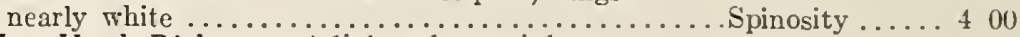

Mrs. Hugh Dickson. A light salmon pink on

cream ground, strong grower................... Spiritual...... 160

Mrs. Routzahn. Buff or apricot ground flushed

and suffused with delicate pink......................... 200

Mrs. Townsend. White edged with blue........... Spissitude.... 250

Nettie Jenkins. Pale lavender mauve.......................... 400

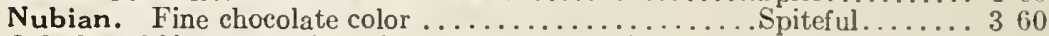

Othello. Of immetıse size, rich maroon. One of

the best dark sweet peas.............................. 200

Primrose. Deep primrose or creamy yellow............. Steadily....... 180

Rainbow. Soft rose color in fine hair lines on white... Steak......2 20

Rosabelle. A very fine large rose-colored flower, giant size and a strong grower ......................... \& 00

Senator. Claret and chocolate stripe on French grey ground, large and most distinct.............. Stocking...... 180

Sterling Stent. A deep salmon; less influcnced by weather than any other sort of its class........Stocks....... 400

Tennant. Purplish mauve, a large, fine flower,

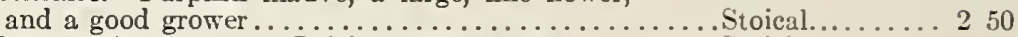

Thomas Stevenson. Bright orange scarlet......... Stoicis........ 250

Vermilion Brilliant. A brilliant scarlet Spencer of splendicl form..................................... 00

Wedgewood. A lovely self blue.............. Stoniness.....6 60

White. Flowers very large and of magnificent Spencer waved form....................... Street....... 1 80

Vaughan's Special Mixture of Spencer Varieti es, made up mostly from separate varieties........ Stattary......2 00

Spencer Hybrids in splendid mixture, $100 \mathrm{lbs} \$ 60.00 \ldots .$. Streator...... 70

\section{Unwin and Grandiflora Types}

America, the brightest blood-red, striped white........ Seide........\$0 45

Aurora, orange rose, striped on white....................... 65

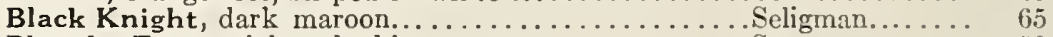

Blanche Ferry, pink and white........................ 50

Captain of the Blues, purplish mauve....................... 100

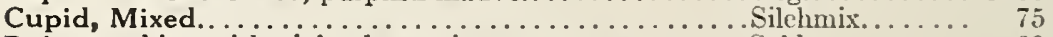

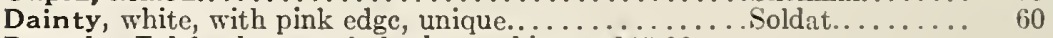

Dorothy Eckford, one of the best whites. $\$ \$ 5.00$ per 100 lbs.......................................... 50

Emily Henderson, white, early and free. $\$ 45.00$ per

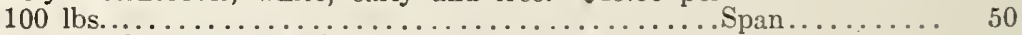

Frank Dolby, largest and finest pale blue. $\$ 90.00$ per

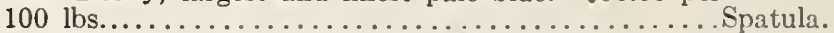

Flora Norton, a very bright blue. $\$ 60.00$ per $100 \mathrm{lbs} .$. Spatz......... 65

Gladys Unwin, pale rosy pink, $\$ 75.00$ per $100 \mathrm{lbs} . .$. . Spearfish....... s0

Helen Pierce, bluc, with dark grain markings......... Spinley ....... so so

Henry Eckford, an extraordinary orange color........ Spinster..... 125

Hon. Mrs. E. Kenyon, yellow......................... 70

King Edward VII, bright red. $\$ 45.00$ per $100 \mathrm{lbs} . .$. sported....... 50

Lady Grisel Hamilton, best of all lavender sorts.

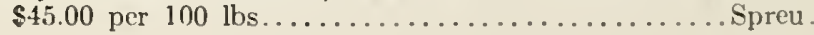




\section{UNWIN AND GRANDIFLORA TYPES-Continued}

Lord Nelson, deeper and richer than Navy Blue; samc Tel. Cipher

as Brilliant Blue............................. Spring...

Lovely, Soft shell pink............................. 60

Miss Willmott, richest orange pink, shaded rose ..... Stafel....... 50

Mrs. Geo. Higginson, Jr., delicate light blue........ Stallman ...... 70

Mrs. Walter Wright, beautiful mauve color ......... Statt ........ 60

Navy Blue, deep violet blue.................. Statthaf...... 65

Nora Unwin, giant white. $\$ 70.00$ per $100 \mathrm{lbs} \ldots \ldots \ldots$. Stattin........ 75

Othello, a beautiful dark brown chocolatc-red color.... Stattlich .... . . 70

Prima Donna, soft pink ..................................... 65

Queen Alexandra, giant size scarlet............... Stellate...... 60

Sadie Burpee, pure white, hooded form ............ Stale. ....... 45

Saint George.................................... 35

Salopian, one of the best of the dark briglit reds....... Stimme...... 60

Senator, chocolate, striped white................ Stock........ 50

Shasta, pure white, large open form............. Stockely ...... 65

White Wonder, the many-flowered white..................... 70

\section{CHRISTMAS or WINTER=FLOWERING SWEET PEAS}

Canary Bird, early yellow ................... Seiutke....... L 100

Christmas Countess $\}$ sky blue................. Spiral .......100

Earliest of All, pink and white................. Siesta ....... 75

Christmas Meteor, brilliant red............................ 120

Christmas Pink, Pink and White...................... I 00

$\left.\begin{array}{l}\text { Christmas Prima Donna } \\ \text { Mrs. F. J. Delansky.... }\end{array}\right\}$ daybreak pink .......... Seed ........ 100

Christmas, White )

Mont Blanc...... pure white................. Saling....... 85

Florence Denzer,

Mrs. Alexander Wallace, lavender................Standery ...... 100

Mrs. E. Wild, bright rose.............................. 100

$\left.\begin{array}{l}\text { Mrs. W. W. Smalley } \\ \text { Mrs. William Sim... }\end{array}\right\}$ salmon pink..............Starost........ 90

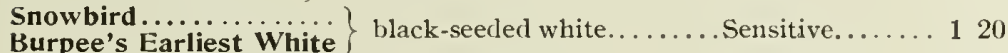

Le Marquis, navy blue .......................... 100

Winter=Flowering Sweet Peas Mixed, $100 \mathrm{lbs}$. $\$ 75.00$. Sentence ..... 80

\section{SWEET PEAS IN MIXTURE}

Vaughan's Prize Mixture-Stumpf. This mixture contains the cream of the new and standard sorts, including most of the new Spencer varieties. It is made up entirely of separate named sorts, carefully proportioned as to its composition, and we can safely say "there is no better mixture in existence," no matter at what price or under what name it may be offered. Per lb. $\$ 1.00,10$ lbs. $\$ 9.50,100$ lbs. $\$ 90.00$.

Vaughan's Florists' Mixture-Stumpfsinn. 'This is a mixture made by ourselves, of principally light-colored varieties, suitable for florists' cut-flower trade. It is a very carefully proportioned mixture and gives satisfaction. Per lb. 75c, per $10 \mathrm{lbs}$. \$7.00.

Eckford Mixture-Stunde. This mixture contains over thirty varieties, inclufing some of the novelties of 1913 and other choice sorts. Per lb. 50c, 100 lbs. $\$ 45.00$.

All Colors Mixed-Sturm. Also a very good mixture. Contains over twenty varieties. Per lb. $45 \mathrm{c}, 100 \mathrm{lbs} . \$ 40.00$. 
Swect Sultan (Centaurea Moschata), mixed .... Supply ...\$0.20 $\$ 2$.

Yellow (Centaurea Suaveolens)........... Cement ... .25

Sweet Vlolet, Semperflorens, blue..................eeper .. .65

2.80

8.00

Sweet Villiam, single mixed......5 lbs., \$4.75 .. Swell .... .10 1.00

Mammoth-Flowered, single mixed........ Swelter ... .25 3.00

Double mixed ................... Swift .... $.75 \quad 9.00$

Double white ....................... swiftness . .50 6.00

Blood-red (Atrosanguineus pl.)........Swiger ... $.60 \quad 80.50$

Mixed .............................................. .30 30

Oculatus Marginatus fl. pl................... .50

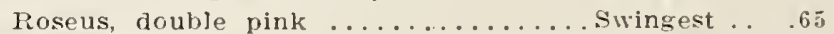

8.011

Single Coppery Red ................. Swipage . . 1.00

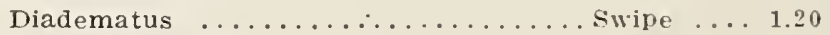

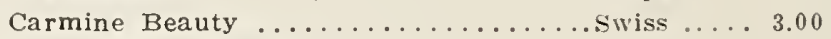

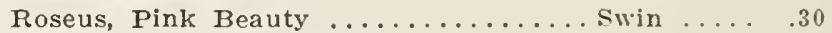

Blood-red (Atrosanguineus) ............... . . .15 1.50

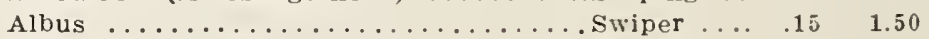

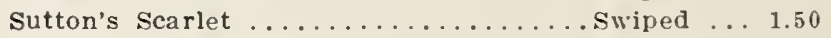

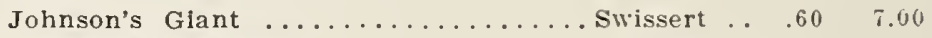

Oculatus Mrarginatus .............. Swinger .. . .10 1.20

Nigrescens, darkest sort ............ Swissing ... .15 1.80

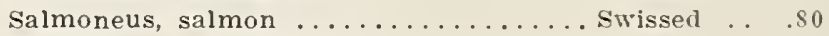

Harlequin (Mrutabilis) ............ Swivel ... .15 1.80

Barbatus $X$ Chinensis annual Sweet

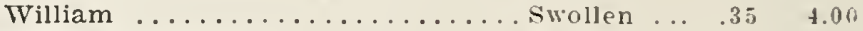

Vaughan's Special Mixture.............. Switch ... .60 7.00

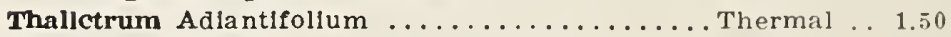

Thunbergla, mixed ................... . 30

3.80

Torenla Fournlerl Grandiflora......1/8 oz., 25c..Torment .. 1.80

White Wings ............ $1 / 8$ oz., 25c..Torpedo .. 1.80

Bailloni .............. 1/8 0z., 65c..Torpilz ...

Tritoma Grandlfiora, new hybrlds, mixed....... Tritoma .. $\quad 45 \quad 5.00$

Valemana Rubra, Garden Hellotrope..........Vase ....\$ \$ 20

Rubra Flore albo, white ........... Vasal .... .20

Vauglan's "SPECIA, Mixtures" contafn only the best selected strains.

Our Asparagus I'lumosus Seed has a high pereentage of germinatfon.

Vauglan' Gladiolus Bubs, home grown. are best and chenpest.

Electrotypes of our catalog illustrations can be furnished at $15 \mathrm{c}$ per $\mathbf{q}$. In. 


\section{VERBENA VAUGIIAN'S BEST MIXTURE. Verbest.}

This is unquestionably the best strain of Verbena seed in existence. It is composed of the choicest strains and colors of the Mammoth type, the best European introductions of late years, ali grown separately and mixed by us in the right proportion. Per oz., $\$ 1.50$; per 1 b., $\$ 20.00$.

TEL. CIPAER.

o7. L.8.

Verbena hybrida, Defiance, brilliant scarlet, true.. Velnage $\ldots \$ 0.75 \$ \$ 9.00$

Firefly, brilliant scarlet, white eye.........vein .....90 12.00

Mammoth, mixed .................... Velveteen...60 6.50

White, extra cholce................ Vemager.. $.65 \quad 8.00$

Purple shades ..................................60 7.60

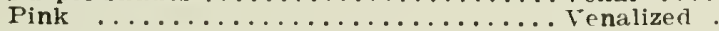

Itallan Striped ................... Vengar ... .60 6.50

Striped, white on red ground, very fine.....vengeance. $.85 \quad 10.00$

Auricula-Flowered, mixed ............................. $50 \quad 6.00$

Mayflower, beautiful pink................Vengehard. $.90 \quad \mathbf{1 2 . 0 0}$

Gigantea, giant flowered in splendid mixture. Venison $\ldots 2.00 \quad 28.00$

Coceinea Stellata, scariet, white eye........Venice ... $1.25 \quad 16.00$

Violaeea Stellata, purple, white eye......... Venitian .. $.85 \quad 10.00$

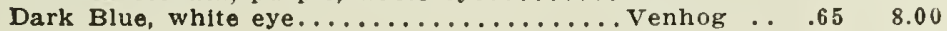

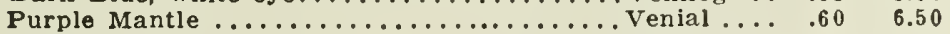

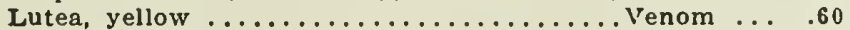

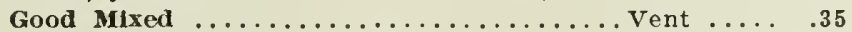

Extra Cholce Mlxed................... Verdan ... .50

Nana Compecta, dwarf mixed......... Verdure .. .85

Venosa ................................ . 30

Lemon (Citriodora) ........................... 1.50

Frinoldes, Moss Verbena, purple..........Vernest .. .30

White .............................. $\$ 0$

Veronica spicata, mixed............... Viands ... .60

Vinca Rosea, mixed..................... Vibrate .. .35

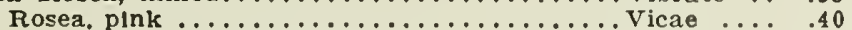

Fl. Albo, white with pink eye........ Victlm ... .40

Alba Pura, pure white............ Victor .... .40

Vlola Cornuta, mixed................ vide .... .45

Admiration, dark blue.......................... 1.00

Golden Yellow ............................. .50

Pure White (White Perfection).................. .80

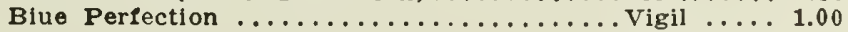

Vlola Odorata, Sweet Violet............................ .65

Virginia Stocks, mixed................. Vigilant .. .10

Viscaria, mixed ...................... Viscaria .. .15

4.00

6.00

10.00

3.50

4.00

4.50

4.60

$\$ .60$

5.00

8.00

.70

1.60

Wahlenbergia, see Platycodon.

Wallfower, single mixed................Wali .... .10

Parislan Forcing, bloom the first year........ Walloping. 15

Double Mixed, extra choice............. Water ... 1.80

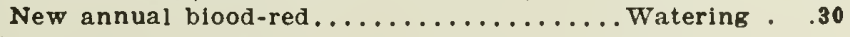

Whitlavia Gloxinoides, blue............Whirl ... .10

Wild Cucumber............10 lbs., \$2.50..Wilder ... .10

Wistarla Sinengig

Winglter 


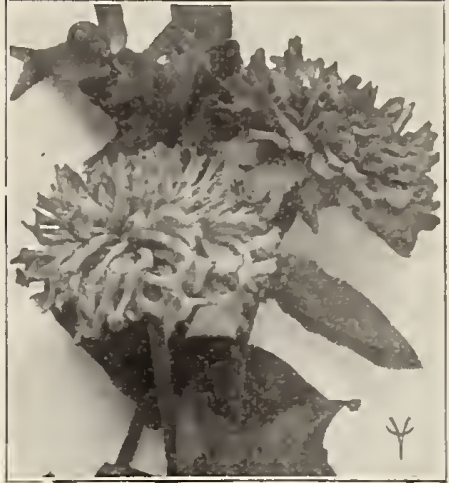

ZINNIA-Crested and Curled

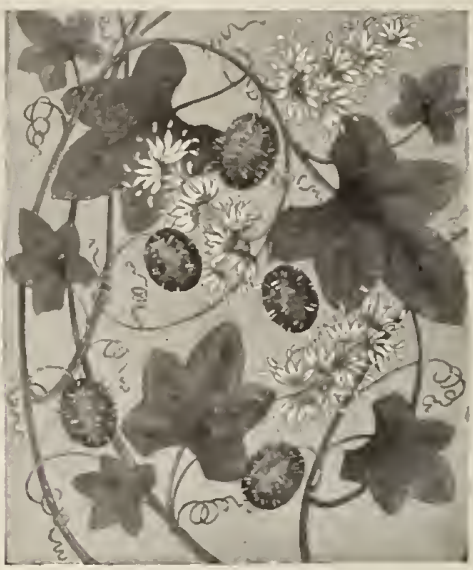

WILD CUCUMBER

TEL. CIPUEH. OZ.

LB.

Xeranthemum, double mixed............. xenia ...\$0.25

$\$ 2.60$

7iea Japonica 1ol. var., Varlegated Maize........ Zealand .. .10

Quadricolor Perfecta ......................... .

1.80

ZInnia Elegans, Double White............ Zealot ... .20

2.40

Elegans Double, Scarlet........................ .20

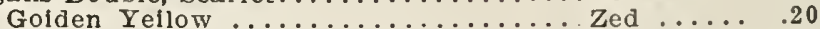

2.40

Pink .................................. .20

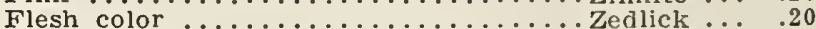

Jacqueminot, double deep blood-red.....Zinhate .. $\quad .25$

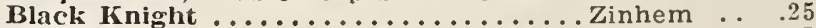

Double mixed ........... 1bs., $\$ 8.50 \ldots$ Zingle ... .15

Double Dwarf (Pumila n. pl.), Mxd., extra...Zion ..... .25

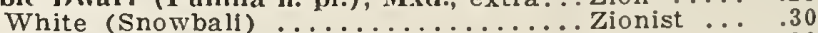

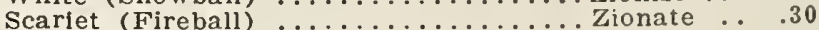

Rose (Pink Ball)........................... .30

2.40

2.40

2.40

2.80

2.80

1.85

2.40

3.00

3.00

3.00

Robusta Plenissima, mixed, double, ex. large. Zither ... . 35

4.00

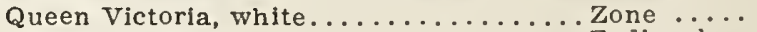

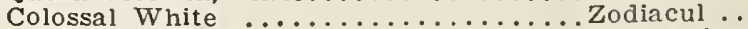

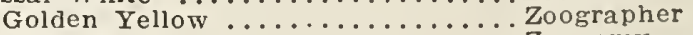

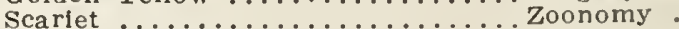

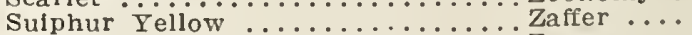

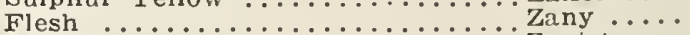

Crimson $\ldots \ldots \ldots \ldots \ldots \ldots \ldots \ldots \ldots \ldots$ Zanist $\ldots \ldots$

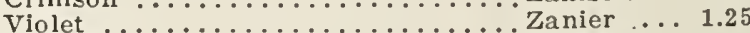

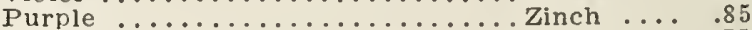

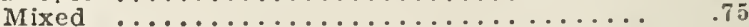

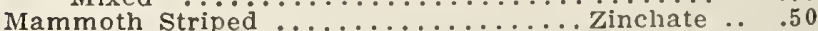

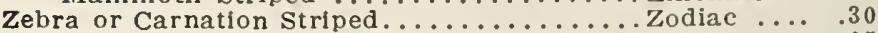

Graclllima coccinea pl. (Red Riding Hood)..Zodactor .. .65

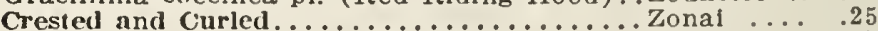

Double Pompone (Darwin) mixed............... Zonave .... .30

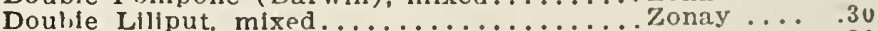

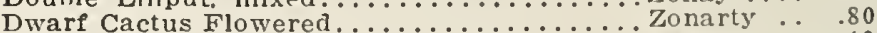

Mexicana (Haageana) fi. pl....................................... 40

Hybrida, new Mexican Hybrids............. Zymo .... .75

3.50

Wild Flower Garden Mixture, 100 lbs., $\$ 30.00 ; 10$

lbs. $\$ 3.50 \ldots \ldots \ldots \ldots \ldots \ldots \ldots \ldots \ldots \ldots$ Winter ... 


\section{SUMTIER FLOWERING, BULBS, ETC. Special Prices on Large Quantities}

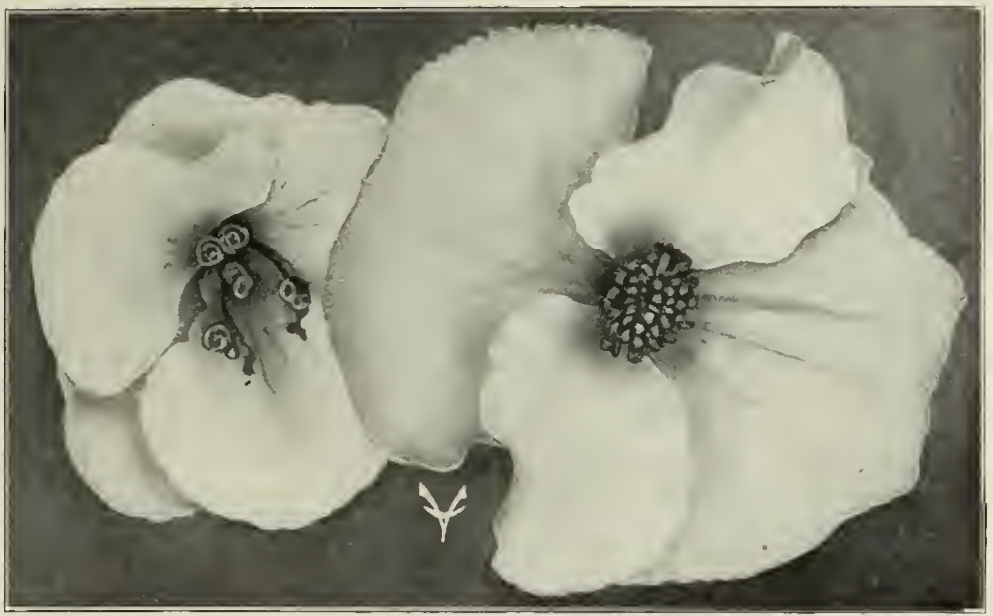

SINGLE TUBEROUS ROOTED BEGONIA

A MARYLLIS.

TEL. CIPHER. DOZ.

100

1000

Belladonia Major ...................... $\$ 1.25 \$ 8.00$

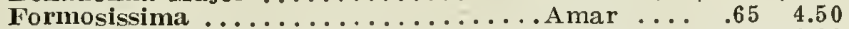

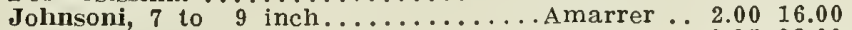

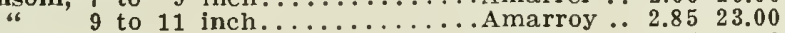

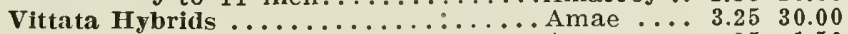

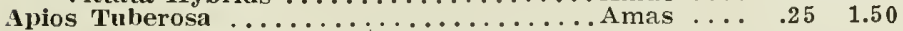

$\$ 40.00$

\section{BEGONIAS.}

Tuberous Rooted Giant Flowering Varieties

We import only bulbs which are grown from the finest, large-flowering strains of seed and which are sure to give satisfaction.

Single Sorts-White, scarlet, pink, yellow, $1 \frac{1 / 4}{4}$ inch and up....................

Single Sorts-Same varieties as above,

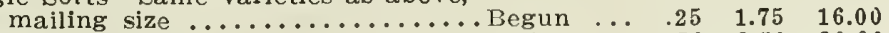

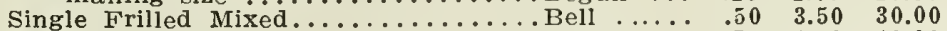

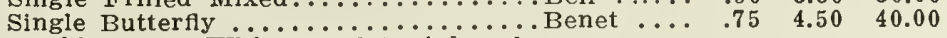

Double Sorts-White, scarlet, pink, yel-

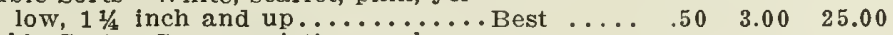

Double Sorts-Same varieties as above,

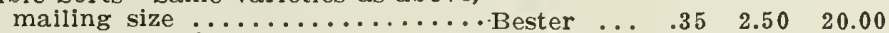

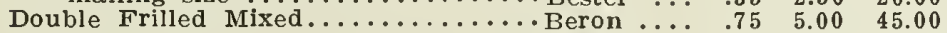

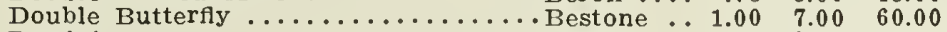

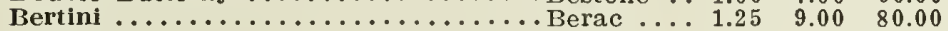

Cristata $\ldots \ldots \ldots \ldots \ldots \ldots \ldots \ldots \ldots \ldots$ Bemol $\ldots \ldots \ldots .15 \quad 8.00 \quad 75.00$

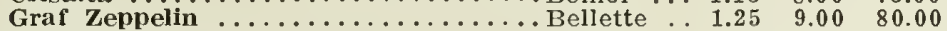

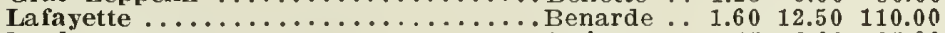

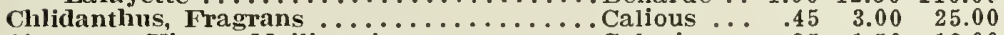

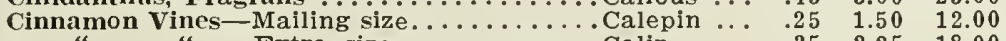

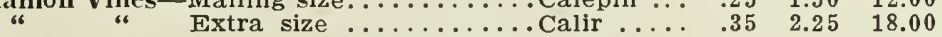

\section{GLOXINIAS.}

Named varieties $\ldots \ldots \ldots \ldots \ldots \ldots \ldots \ldots$. Callat $\ldots . .50 \quad 30 \quad 3.00 \quad 25.00$

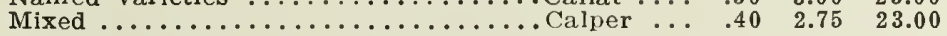

Vaughan's "SPECIAY Mixtures" contain only the. best sclected strains. 


\section{SUMMER FLOWERING BULBS (Continued).}
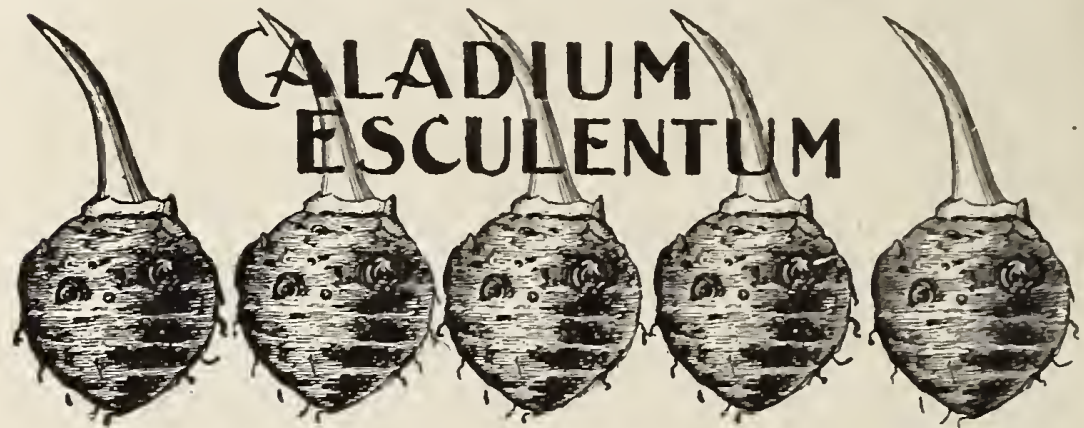

\section{CALADIUM ESCULENTUM, or Elephant's Ear.}

Cured and sound bulbs with good, live center shoots.

TEL. CIPHER. DOZ. 100.1000.

5 to 7 inches in circumference..... Calamine $\ldots \ldots \$ 0.25 \quad \$ 1.50 \$ 12.00$

7 to 9 " $\quad$ " $4 .$. Calandre .... .50

9 to 11 " "

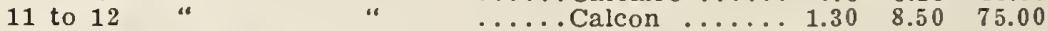

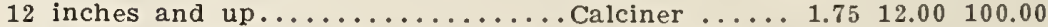

Ten per cent. diseount from above when slupped from New York Store.

Callas Elliottiana, first size, yellow ....Gab ....... 2.00 15.00

Fuliottiana, mailing size, yellow.. Gabe ........ 1.5012 .00

Spotted......................... $.50 \quad 3.00$

Fern Balls, 5 inches in diametel...... Gaze ........ 1.8014 .00

" " 7 inches in diameter.......Gazer ....... 2.8022 .00

Hyacinthus Candieans, $1-2$ inches..... Gazism ....... . . $20 \quad 1.25$

" $\quad 2-3$ inches.......Gazeler ......

Ismene Calathina ................. Liberal ........60 $4.00 \quad 35.00$

Madeira Vine Roots, $2-3$ inches.........Liberaler .... .25 $1.50 \quad 12.00$

" " " 3 inch up........Libertin ............

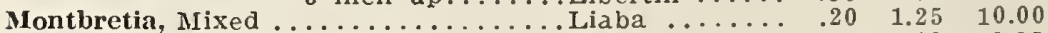

Oxalis, White, Pink and Red...........Liab ....... $\quad .40 \quad 2.25$

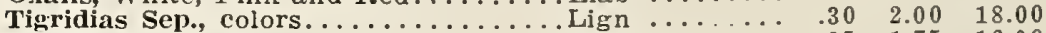

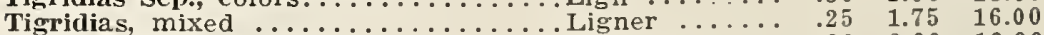

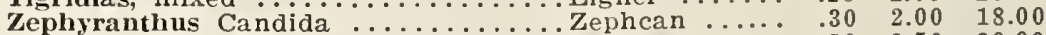

Zephyrauthus Rosea .............. Zephcaner .... $.50 \quad 3.50 \quad 30.00$

Well Cured Bulbs.

TUBEROSES.

TEI, LIPFER.
Free from Rot

100. 1000 .

True Excelslor Pearl, 1st Class, as received

“" 4

from our growers

" in New York........ Rose ....\$0.\$0 \$7.50

" in Chicago.........Rosebif ... $90 \quad \$ .00$

Hand picked after March 1st...... Roseat. Write for price

“ malling size...................... . .50 4.50

in New York Roseau ... 45

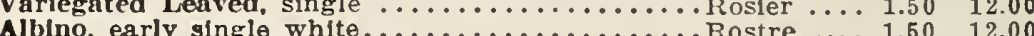

Armestrong's Ever Hlooming................ Rotule .... 1.7515 .00

Vaughan's International Pansies should be carried by all dealers. 


\section{SUMMER FLOWERING BULBS}
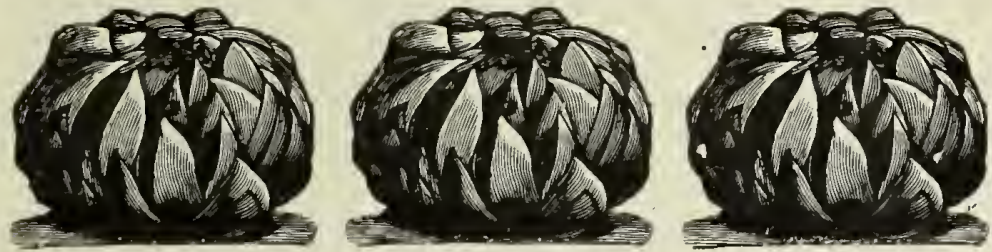

\section{HARDY LILIES}

PLEASE NO'TE-Japan Lily Bulbs usually arrive in this country with a large percentage of them rotten and entirely worthless. We repack all of this stock before shipping and bulbs will be in sound condition when leaving our store.

Prlces below subject to change without notlce.

We furnlsh 25 of a klnd at the 100 rate, less at the dozen rate.

TEL. CIPHER. DOZ. 100. 1000.

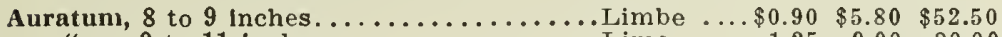

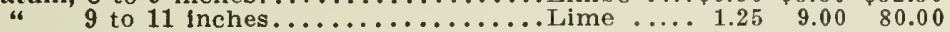

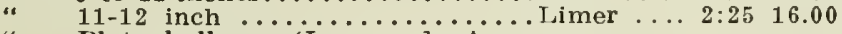

"Platyphyllum (Improved Aura-

tum) $8-10$ inch.................... 2.00 15.00

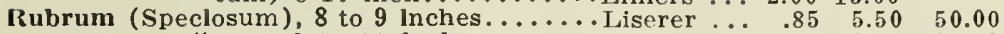

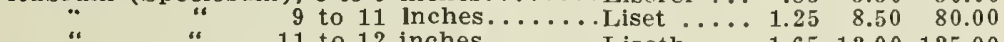

Album $\quad \because \quad 11$ to 12 inches........ $\quad 8$ Lisoth $\ldots .1 .6513 .00125 .00$

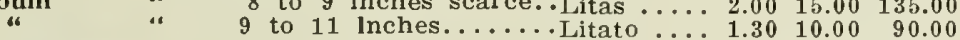

Canadense ................................................. . . . 450 4.50 40.00

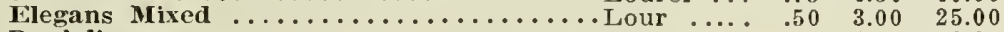

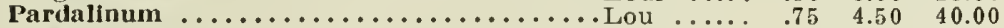

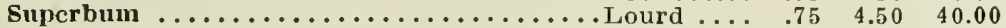

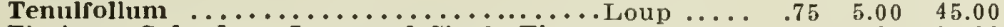

Tigrinum Splendens, Improved Single Tiger. Literary . $\quad .50 \quad 3.00 \quad 25.00$

"Fl. Pl.. Double Tlger Llly...........izard .... $.50 \quad 3.00 \quad 25.00$

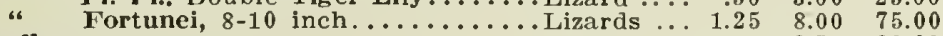

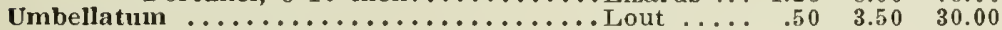

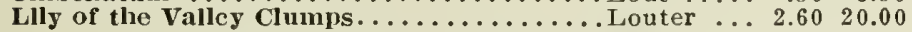

\section{LILY BULBS AND VALLEY FROM ICE STORAGE}

These are Chicago storage prices. Customers of our New York house will please ask for prices there. Our cases are numbered and "stock marked," and these records show on your invoice. You can thus "Repeat" your orders with assurance of receiving the same grade and packing. Try us. Book now for future dates and secure your supply.

\section{LILY OF THE VALLEY PIPS}

Packed 500 Pips to case......................... 8.50

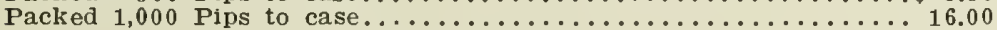
Packed 2,000 Pips to case........................ 30.00

\section{Lilium Rubrum}

8-9 inch (170 Bulbs to case) 9-11 inch (100 Bulbs to case).

Lilium Auratum

Per case

$8-9$ inch (160 Bulbs to case) $\$ 9.00$ 9-11 inch (100 Bulbs to case). 9.00

\section{Lil Giganteum}

Per case

7-9 inch ( 300 Bulbs to case) $\$ 15.00$ 8-10 inch ( 225 Bulbs to case) 18.00 $9-10$ inch ( 200 Bulbs to case) 18.50 


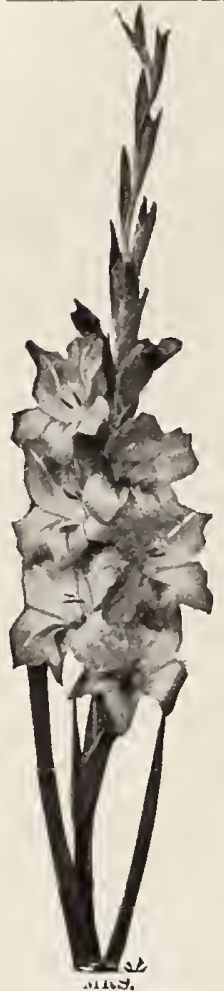

FRANCIS KING

\section{GLADIOLUS}

\section{MRS. FRANCIS KING}

The Leading. Decorative Gladiolus-For the Trade

The long, strong flower stalks with foliage, its effective fower spike with a good line of reserve buds continually

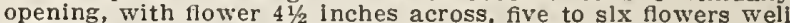
spread out on the spike at the same tlme, its brilliant vermilion scarlet color gives for vases, in hotel lobbies and dining rooms, parlor decorations, etc., an effect not produced by any other Gladiolus.

FIRST size, per 100, $\$ 1.00$; per $1,000, \$ 8.50$, Mailing size, per 100, 80e; per 1,000; $\$ 7.00$. Code Vur'd-Gam."

\section{CHICAGO WHITE}

The Eurliest White

The flowers are well expanded, iell placed upon the stalk, pure white with faint lavender streaks in the lower petais. In form they resembie the Childsli type. They are borne on tail, straight stems and from five to seven flowers are open at one time. It is one of the earlest varietles to bloom.

We gave this a very effective try-out the past season among some of the most important forcers of bulbs for cut flowers in and about Chicago. The results have been very satisfactory and a fixed demand for this variety for early use, both in doors and out of doors. A notable feature is the fact that many of these bulbs grown and sold by us produce two flower spikes.

FIIRT size, per 100, \$3.00; per 1.000, \$25.00. Mailing size, per 100, \$2.50; per 1,000, \$20.00. Code Word"Garden,"

\section{AMERICA}

Soft lavender pink, very light, almost à tinted white, spikes two and three feet long, very large spreading blooms and luxuriant dark green foliage. When cut it lasts in water a week or more, the blossoms retaining their vigor and delicate coloring to the last.

FIRST size, per $100, \$ 1.25$; per $1,000, \$ 10.00$. Mailing size, per $100, \$ 1.00$; per $1,000, \$ 8.50$. Code Word"Gladamer."

\section{MARGARET}

A vigorous grower 5 to 6 feet, with broad foliage. The spike is straight and strong. The flowers are large, beautifully arched and arranged in two rows facing the same way, with 6 to 8 open at a time. The color is a brilliant carmine with a large white blotch on lower petals, the white and carmine blending into a violet tint. FIRST size, per 100, \$10.00. Code Word- "Gardenite."

\section{VAUGHAN'S PRINCEPS}

The flowers are 5 to $6 \mathrm{in}$. broad, the petals are very wide and rounded, well reflexed, forming an almost circular flower. The color is brilliant scarletcrimson, carrying mostly three broad white blotches on the lower petals.

Per 100, $\$ 3.00$; per 1,000, $\$ 25.00$. Code Word-“Garcia."

\section{NIAGARA}

In type the variety resembles "America," but the flowers a ppear to be somewhat larger, measuring $4 \frac{1}{2}$ inches across. In color the fowers are a delightful cream shade with the two lower inside petals or segments blending to canary yeilow. The throat is splashed with carmine, and the lower ends of the outside petals are also blushed with carmine. The stamens are purple and the stlgmas pale carmine, this little addition in the coloring relieving creamy effect of the petals.

FIRST size, per $100, \$ 1.00$; per $1.000, \$ 35.00$. Code Word-"Gauble."

\section{KUNDERDI "GLORY"}

New Type of Gladioli Witl "Rufrled" Petals.

The broadly expanded, wlde open paired flowels, all face in the same direction and are carried on straight, stout stalks, fully $31 / 2$ feet. From three to eight of these handsome flowers are open at one time. Each petal is exqulsitely ruffied and fluted.

The color is a dclicate plnk, with a most attractive crimson stripe in the center of each lower petal, the shade of which is unique in Gladioli. The ruffling of the petals in this new straln has attracted attentlon the country over.

FIRST size, per 100, $\$ 4.50$; per 1,000 , \$10.00. Code Vord-“Garg." 


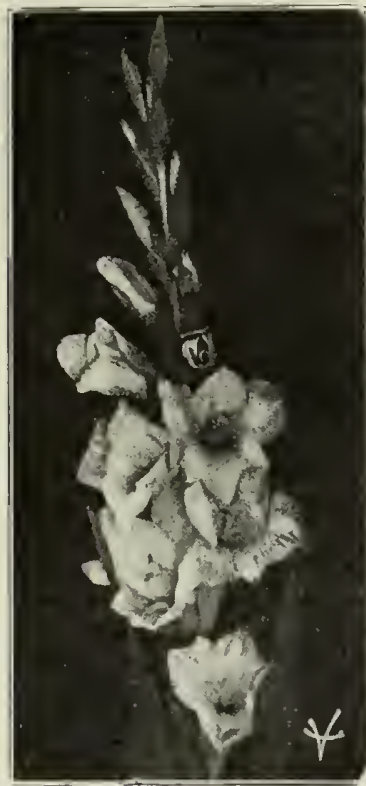

HYUE PAKK.

\section{GLADIOLUS - Continued}

\section{HYDE PARK}

\section{VERY EARLY BLOOMER}

A new hybrid of the Gandavensis type, a cross between May and the old favorite Shakespeare.

The flowers have the same fine open shape and large size as Shakespeare, with the same exquisite coloring in the three upper petals - a frosty, sparkling white, penciled and feathered with light and dark rose-but the lower petals are much more distinctly blotched with cream-which is an added charm.

The foliage is clean and healthy, remaining deep green until frost.

It is a very early bloomer, making it of special value in the cut-flower market, as it comes in at a time when there is very little competition from other varieties; an ideal variety for Florist use.

Received certificate of merit from American Institute of New York City.

Priee-Per 100, $\$ 5.50$; per 1,000, $\$ 50.00$.

\section{PANAMA}

A new seedling of America which resembles the parent variety in every way except that it is a much deeper pink. A grand variety which evokes words of praise wherever exhibited. Spike very long with flowers large and well arranged. Price-First Size, per $100, \$ 7.00$; per $1,000, \$ 65.00$.

\section{AUGUSTA}

Pure white, with slight pink shade on lowel half of the petals, blue anthers; bulb often produces two or more spikes.........First size...\$0.25

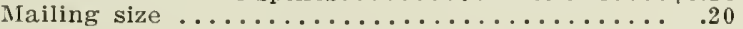

Baron J. Hulot. Dark velvety purple.......... .50

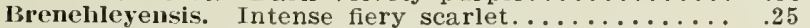

Brilliant. Beautiful orange-red, with carmine and white throat $\ldots \ldots \ldots \ldots \ldots \ldots \ldots \ldots \ldots \ldots \ldots . . . \ldots$ Canary IBird. Very fine clear canary yellow....... .85 Ceres. Pure white, spotted with purplish rose..... .35 Chrysolora. Golden yellow................. 50 Columbia. Light orange-scarlet, freely blotched and penciled with blush purple............ .50

Contrast. Color intense scarlet with large pure

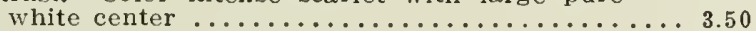

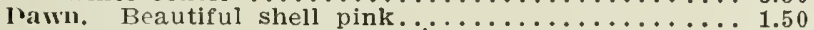

Emma Thursby. White striped and blotched....... .50

Eugene Scribe. Tender rose, blazed carmine-red.... .50

Enlope. Best pure white yet introduced ......... 4.00

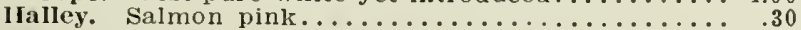

Henry Gilman. Deep salmon-scarlet with pure white band $\ldots \ldots \ldots \ldots \ldots \ldots \ldots \ldots \ldots \ldots \ldots . . .60$

Isaae S. Hendrickson. Large handsome spikes, color an irregular mottling of white and pink.... .75

Isaae 3uchanan. Very fine yellow............ 75

Jessie. Rich velvety red, early bloomer......... . 50

Kate. Blush white with crimson blotched throat.... .50 Klondyke. Light yellow crimson maroon blotch..... .50
$100 \quad 1000$

$\$ 1.25 \$ 10.00$

$1.00 \quad 8.50$

$3.00 \quad 25.00$

1.2510 .00

3.50

$6.00 \quad 50.00$

$2.00 \quad 16.00$

$3.50 \quad 30.00$

$3.50 \quad 30.00$

25.00

12.00

3.00

3.25

30.00

1.75 i5.00

$4.00 \quad 35.00$

$5.00 \quad 45.00$

$5.00 \quad 45.00$

$3.00 \quad 25.00$

$3.00 \quad 25.00$

$3.00 \quad 25.00$ 


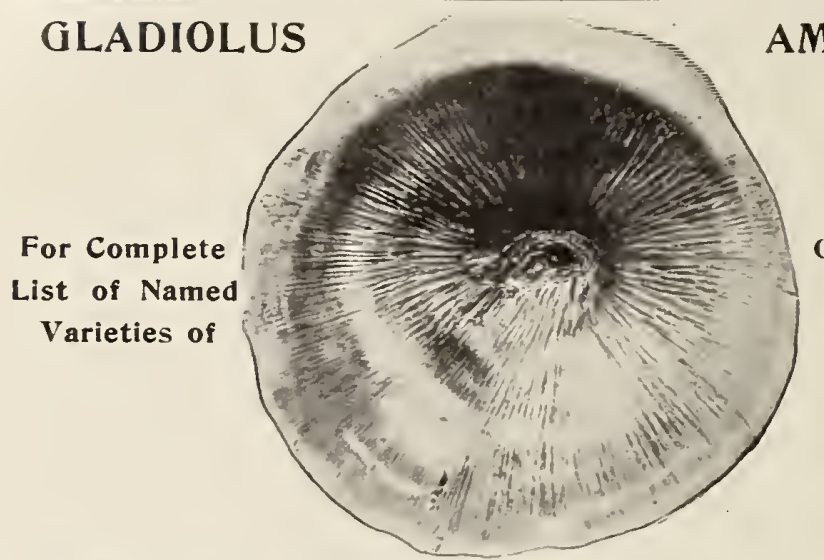

Gladiolus, See

Our Book

for Florists.

Madame Monneret. Delicate rose .............\$0.45

May. Pure twite, finely flaked rosy crimson....... .45

Melrose. White flaked pink, bright crimson center.. 1.50

Mrs. Beecher. Deep rosy crimson, with pure white throat freely marked and spotted.............

Mrs. Frank Pendleton. Immense sized fower, delicate salmon pink with blood red blotch in throat.... 2.25

Mohonk. Deep dark pink with spotted throat, shaded

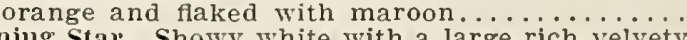

Morning Star. Showy white with a large rich velvety crimson throat; very early to bloom......... 1.25

Nezinseott. Bright scarlet with crimson black .... $\quad .75$

Pink Beauty, Violet Rose, dark center............ .35

$17.50 \quad 150.00$

Reine d'Anjou. Pure white with very small purple streak at the bottom of the throat.......... 1.25

Salem. Salmon pink, maroon blotches.......... $\quad .50$

Seribe. Beautiful rose, tinted white, striped carmine .75

Shakespeare. White tinged rose............. .50

Sulphur King. Clear sulphur yellow........... 1.30

Velvet King. Dark scarlet................ $\quad .35$

Wild Rose. Bright rose with blush tint............ 1.00

Wm. Falconer. Beautiful clear, light pink........ .80

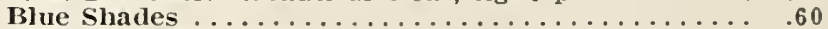

Lilac and violet shades ................... $\quad .50$

Pink shades ........................ .25

Pink and white shades.................. .40

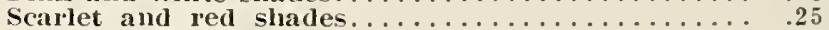

Searlet with white throat................. .35

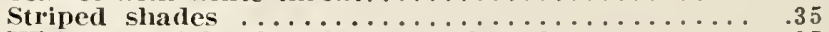

White and light shades, extra selected......... $\quad .35$

White and light shades, $\mathbf{x x} \ldots \ldots \ldots \ldots \ldots \ldots \ldots .20$

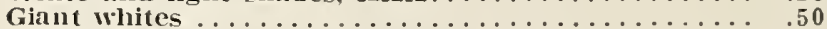

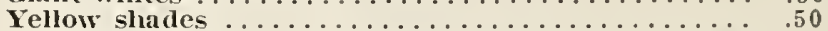

5.0045 .00

$8.00 \quad 75.00$

$5.00 \quad 47.50$

$1.75 \quad 15.00$

$8.50 \quad 80.00$

$3.50 \quad 30.00$

$4.50 \quad 40.00$

$3.25 \quad 30.00$

$9.00 \quad 85.00$

$2.50 \quad 22.50$

7.00
60.00

$5.50 \quad 47.00$

$4.00 \quad 35.00$

$3.50 \quad 30.00$

$1.60 \quad 14.00$

$2.75 \quad 23.00$

$1.35 \quad 10.00$

$2.25 \quad 20.00$

$2.25 \quad 20.00$

2.2520 .00

$1.60 \quad 13.50$

$3.50 \quad 30.00$

Rainbow Mixture, Composed of the choicest named $3.00 \quad 25.00$ varieties and including also the famous Childsii, Lemoine's and Nanceanus sorts. The best possible mixture, and without an equal in America

Extra Fine Mixed. Especially grown for us, which we further improve by the addition of many choice new kinds, including Childsii, insuring a wide range of colors, shades and types......

Good Mixed. This mixture contains a very complete range of colors.

First Size $\ldots \ldots \ldots \ldots \ldots \ldots \ldots \ldots \ldots \ldots \ldots \ldots \ldots \ldots . . \ldots \ldots$ 


\section{GREENHOUSE DEPARTMENT.}

Note: On all orders for dormant stock, such as Roses, Harly climbers, Shrubs, Fruits and Trees, packing will be charged at cost.

Besides the few items mentioned below we have Shrubs and Perennials in large quantities in all the popular and best commercial varieties. Ask for our Book for Florists which has a complete list. Special quotations will be made on large orders.

HYDRANGEA PANICUTATA GRANDIFLORA.

Our large stock of this is strong, bushy, and has good roots.

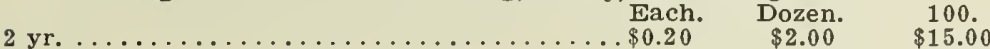

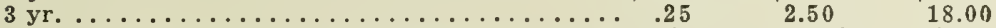

Standards, $11 / 2$ to $2 \mathrm{ft}$. stem............... (Prices on application.)

OTHELLO PLUM.

The finest purple-leaved ornamental on the market.

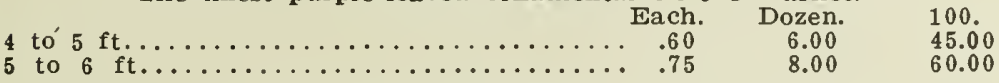

PEONIES.

Our own growing, strictly true to name with from 3 to 5 eyes. Ask

for complete list. Prices on larger clumps on application. Unnamed-in separate colors, 3 to 5 eyes.

Each. Dozen. 100.

Double Pink, Red, and White ........\$ \$0.15 $\$ 1.50 \quad \$ 10.00$

Double Mixed $\ldots \ldots \ldots \ldots \ldots \ldots \ldots \ldots \ldots \ldots \ldots \ldots . . \ldots . . .10 \quad 1.00 \quad 6.00$

Case Lots.

Specially packed cases of roots with 3 to 5 eyes, each with 67 double white, 66 double red, 66 double pink, 200 in all.

200 roots for $\$ 18.00 ; 2$ cases for $\$ 35.00$. We cannot divide cases.

VINES.

Ampclopsis Veitchi-Japan or Boston Ivy.

Each. Dozen. 100.

Strong, 2 year.................. $0.20 \quad 1.75 \quad 12.00$

Strong, 3 year..................... $25 \quad \$ 2.00 \quad 15.00$

Clematis, Large Flowering.

Beauty of Worcestcr, sky blue Gypsy Queen, pur'ple

Ducliess of Edenberg, double white Jaekmani, royal purple

Henryii, white

Mme. B. Veillard, rose shaded lilac Ville de Lyon, red

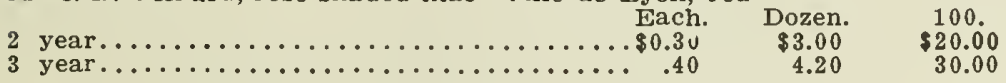

Clematis Panieulata, Small Flowering, white.

Each. Dozen. 100.

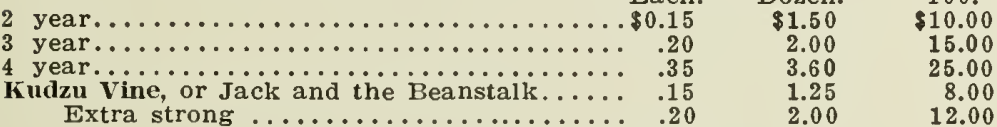

Matrimony Vine. (Lycium Barbarum.)

Each. Dozen. 100.

2 year..........................\$0.15 \$1.50 \$10.00

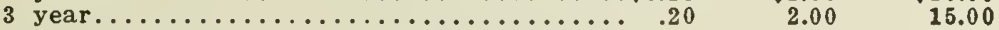

DORMAN'T ROSES.

Not less than 3 of a kind sold at the dozen rate, nor 25 at the 100 rate. Unless noted all are two year, field grown and budded, strong and bushy.

Each, 20c; dozen, $\$ 2.00 ; 100$, \$14.00.

Alfred Colomb, carmine red

Anna de Diesbaeh, rose

Baron de Boristettin, dark red

Baroness Rothschild, pink

Frau Karl Druschki, white

General Jacqueminot, crimson
John Hopper, crimson

Magna Charta, deep pink

Mrs. Jolun Laing, pink

Paul Neyton, deep rose

Prince C. de Rolın, crimson

vlrieh Brunner, crimson 


\section{DORMANT ROSES-Continued.}

Prices, except where noted: Eaeh, 20e; Dozen, \$2.00; 100, \$15.00. American Beauty, Deep pink
Captain Christy, Flesh

Carolina Festout, Pink

Fisher Holmes, Crimson

Iugli Diekson, Crimson

J. B. Clark, Scarlet

Kiaiserin Augusta Victoria, White
La France, Bright satin pinli

Mabel Morrison. Pure white

Mme. Plantier, Pure white

I. P. Wilder, Bright red

Persian Yellow, Golden yellow

Victor Verdier, Deep rose
Each

Gen. Mac Arthur, Crimson.......... . . 25

J. L. Mock, Carmine pink ....... .25

Killarney, pink ............... .25

Lady Ashtown, Pink arid yellow.... . . 25

Mrs. Aaron Ward, Golden orange... . .25

Prince de Bulgarie, Pink ........ .30

Sour. de L. Malmaison, Flesh pink.. .25

White Killarney ............... 30

Rugosa Alba and Rubra, 2 yr., each, $20 \mathrm{c}$
Avoca, Crimson scarlet ........
CLIMBERS.

Alberie Barbicr

American Pillar

Count Zepplin

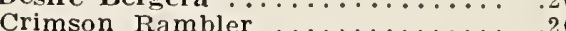

Deliglrt

Dorothy Perkins, pink ........ .20

Excelsa, crimson .............. .25

Flower of Fairfield, crimson...... .25

Hiawatha (single), deep red...... .25

Lady Gay, pink ............. .20

Philadelphia Rambler, crimson .. $\quad .25$

Prairie Queen, bright red....... .20

South Orange Perfection ......... .20

Tausendschon, pink ......... .25

Universal Iavorite .............

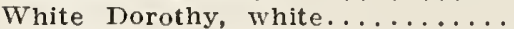

Wichuriana, white
Desire Bergera
2 - Year

.20

.25

.20

\section{BABY RAMBLERS.}

Crimson Baby ............. .20

Erna Teschendorff (scarlet) ..... .25

Jessie (bright crimson) ...........

Mud Jules Gonehault (New), vermillion and orange, changing to pink when matured........... Orleans (pink and white)....... Phyllis (pink)

\section{CANNAS.}

;oz., $\$ 2.00 ; 100, \$ 15.00$.

$5.00 \quad 35.00$

$2.00 \quad 16.00$

$2.00 \quad 16.00$
100

Doz.

3.50

2.50

2.50

2.50

2.50

2.50

3.00

2.50

3.00

$\$ 18.00$

25.00

18.00

18.00

18.00

18.00

18.00

20.00

18.00

20.00
100 Each $\begin{aligned} & 3-\text { Year } \\ & \text { Doz. } 100\end{aligned}$

$\begin{array}{ccccc}\$ 2.00 & \$ 14.00 & \$ 0.25 & \$ 2.25 & \$ 16.00\end{array}$

$2.50 \quad 16.00$

$2.00 \quad 15.00$

$2.00 \quad 15.00$

$2.00 \quad 15.00$

$2.00 \quad 15.00$

$2.00 \quad 15.00$

$2.50 \quad 18.00$

2.2516 .00

$2.50 \quad 18.00$

$2.00 \quad 15.00$

$2.50 \quad 18.00$

$2.00 \quad 15.00$

$2.00 \quad 15.00$

$2.25 \quad 16.00$

$2.00 \quad 15.00$

2.2516 .00

$2.00 \quad 15.00$

2-Year

Doz.

2.50

2.50

100

14.00

18.00

18.00

.25

2.50

18.00

$\begin{array}{lll}.25 & 2.50 & 18.00\end{array}$

$\begin{array}{lll}.25 & 2.50 & 18.00\end{array}$

$\begin{array}{lll}.30 & 3.00 & 20.00\end{array}$

$\begin{array}{lll}.25 & 2.50 & 18.00\end{array}$

$\begin{array}{lll}.25 & 2.50 & 18.00\end{array}$

$.25 \quad 2.50 \quad 18.00$

$.25 \quad 2.50 \quad 18.00$

.25

$2.50 \quad 18.00$

...

3-Year

Each Doz. 100

$\begin{array}{lll}.25 & 2.50 & 16.00\end{array}$

$\begin{array}{lll}.30 & 2.75 & 22.00\end{array}$

$\begin{array}{lll}.30 & 2.75 & 20.00\end{array}$

We are headquarters. All are leading varietles. Full llst on appllcation. For heights, description, etc.. see our Book for Florists.

Prices quoted are for both Dormant 1-2 Eyed Bulbs and 3 -inch pot plants. Dormant Roots are offered up to April 1 or whlle stock lasts. after whlch pot plants are ready. If wanted in large quantity, we wlll quote special prices. Send us your list.

\section{RED WLOWERING-GREEN FOLIAGE Doz.}

Dorm
E Doz.
$\$ 0.60$

Beaute Poitevlne, $31 / 2 \mathrm{ft}$, dark crimson... $\$ 0.60$
Chas. Henderson, $4 \mathrm{ft}$. Brlght crimson... .60 $\begin{array}{lll}\text { Chas. Henderson, } 4 \text { ft. Brlght crimson... } & .60 \\ \text { Comte de Sach, } 4 \text { ft. scarlet crlmson } & .50\end{array}$

\section{$\begin{array}{lll}.25 & 2.50 & 18.00\end{array}$ \\ $\begin{array}{lll}.25 & 2.50 & 18.00\end{array}$}


CANNAS-(Continued).

Dormant roots

Doz. 100

Duke of Marlborough, maroon...... .50

Tulius Koch, 3 ft. brilliant blood red.... 2.00

Fircbird (New) Clear glistening scarlet without any streaks or biotches... 6.50

Milwaukee, $4 \mathrm{ft}$. Dark maroon ....... .60

New Chicago, $41 / 2$ ft. Brilliant scarlet. 1.50

Prince Wied, $3 \mathrm{ft}$. Fiery, velvety blood red .50

The Express, $2 \frac{1}{2} \mathrm{ft}$. Scarlet crimson....

BRONZED LEAVED VARIETIES.

.60

Dormant

3.00

15.00

100 3-inch pots.

Each Doz.

100

\begin{tabular}{rrrr}
50.00 & .60 & 6.50 & 50.00 \\
4.00 & .10 & .85 & 6.00 \\
10.00 & .15 & 1.50 & 10.00 \\
3.00 & & & \\
4.00 & .10 & .85 & 6.00 \\
roots. & \multicolumn{3}{c}{3 -inch pots. }
\end{tabular}

Discolor, $6 \mathrm{ft}$. Foliage effect only.....\$0.50

David Harum, $31 / 2 \mathrm{ft}$. Vermilion scarlet .60

Egandale, $4 \mathrm{ft}$. Currant red ......... $\quad .60$

Leonard Vaughan, $41 / 2 \mathrm{ft}$. Bright scarlet .60

$W m$. Saunders, $3 \frac{1}{2}$ ft. Deep rich scarlet .85

Gladiflora, $3 \frac{1}{2} \mathrm{ft}$. Crimson, edged yellow 1.25

IIalley Comet, $4 \mathrm{ft}$. Scarlet, edged yellow 1.25

Mad. Crozy, $31 / 2 \mathrm{ft}$. Vermilion with gold

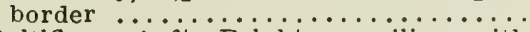

Multiflora, 4 ft. Bright vermilion with

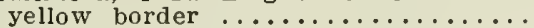

Niagara, $3 \frac{1}{2}$ ft. Deep Crimson with

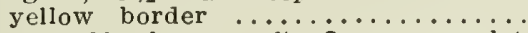

Queen Charlotte, $4 \mathrm{ft}$. Orange scarlet

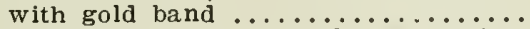

Souv. d'A. Crozy, $4 \mathrm{ft}$. Crimson with

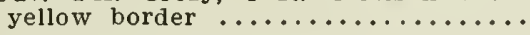

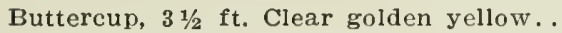

Dwarf Florence Vaughan, 3 ft. Yellow

spotted light crimson
Florence Vaughan, 5 ft. Yellow spotted crimson $\ldots \ldots \ldots \ldots \ldots \ldots \ldots \ldots \ldots \ldots$. . . . . . .

Irungaria, $31 / 2$ ft. Purest La France pink
Irs. Alfred Conard, $4 \mathrm{ft}$. sal mon pink

Venus, $3 \frac{1}{2}$ ft. Rosy pink, mottled

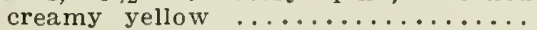

.50

$\$ 3.00$

4.00

4.00

4.00

6.00

8.00

8.00

Each Doz.

100

$.10 \quad .75$

5.00

.10

.75

5.00

.10

.75

5.00

.15

1.25

8.00

.15

1.25

8.00

1.25

8.00

3.00

.10

.75

5.00

.60

4.00

.10

.85

6.00

$.50 \quad 3.00$

.60

4.00

.10

.85

6.00

.60

$4.00 \quad .10 \quad .85$

6.00

.75

5.00

.10

1.00

7.00

.85

6.00

.15

1.25

8.00

.60

4.00

.10

.75

5.00

2.00
2.00

18.00

.20

$2.00 \quad 18.00$

15.00

.20

$2.00 \quad 15.00$

$.60 \quad 4.00$

.10

$.75 \quad 5.00$

3-inch pots.

ORCIID FLOWEIRI CANNAS. Dorinant roots.

Allemania, 4-5 ft. Green foliage, scar- Doz. let and yellow ............... .50

100

Each

Doz.

100

Burbank, $5 \mathrm{ft}$. Green foliage, clear yellow

Frederic Benary, 5-6 ft. Green foliage, red, yellow throat and band.......

King Humbert, 4 ft. Bronze foliage,

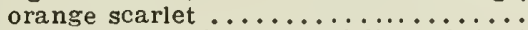

Long Branch, $5 \mathrm{ft}$. Green foliage, bright crimson bordered yellow ..........

Louisiana, $6 \mathrm{ft}$ Green foliage, large

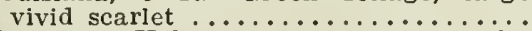

Mrs. Karl Kelsey, $6 \mathrm{ft}$. Orange scarlet

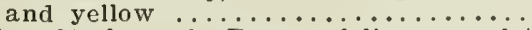

New York, 5 ft. Bronze foliage, scarlet

Wyoming, $7 \mathrm{ft}$. Golden yellow foliage

3.00

.10

.75

5.00

5.00

.60

4.00

.10

.75

5.00

.75

5.00

.10

.85

6.00

.75

5.00

.10

.85

6.00

.50

$$
3.00
$$

.10

.75

5.00

.60

$$
4.00
$$

4.00

.10

$.7 \overline{5}$

5.00

4.00

.10

.85

6.00

5.00

$8.00 \quad .15$

.75

$1.50 \quad 10.00$

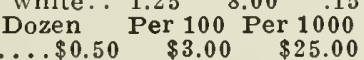

Red Shades Green Leaved......\$0.50

All Shades Green Leaved....... .50

All Shades Bronze Leaved...........50

$3.00 \quad 20.00$

$3.00 \quad 25.00$

\section{DAHLIAS.}

Our Selection of Sorts.
Divided

Fleld Roots, per 100

Named Red, Pink or Yellow..............\$6.00

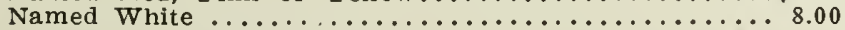

Double Dahlias, mixed, per 100 field roots, $\$ 6.00$.

Field

Roots,

per 100

$\$ 10.00$

15.00 


\section{Garden and Farm Tools.}

Terms: 1st of Month Following Date of Involce.

We Do Not Open New Accounts on thls Stoek Under \$25.00. Please Accompany Order under that amount with Cash.

we Charge Cartage on Orders for Less tlian Tliree Machlnes at a Time. DRILLS AND SEEDERS.

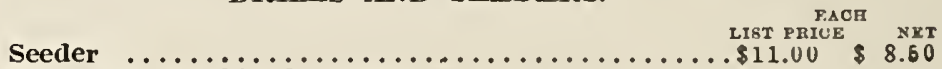

No. 6 Iron Age Combined Dcuble Wheel $\ddot{H o e}$ Hill and

Drill Seeder .............................. $12.00 \quad 9.50$

No. 4 Iron Age Combined Double vicel Hoe and Drill

No. 4 Iron Age Drill Seeder Attaehment.............

No. 6 Iron Age Hill and Drill Seeder Attaehment.......

No. 15 Iron Age Combined Single Wheel Hoe, Hill and

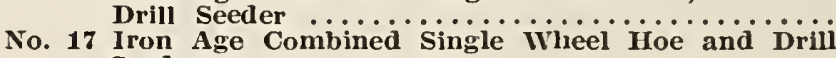

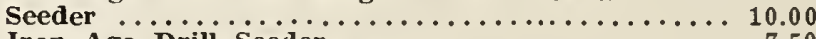

No. 18 Iron Age Drill Seeder................ 7.50

No. 8 Iron Age Itill and Drill Seeder.............. 11.00

No. 22 Iron Age Combined Fertilizer Distributer, Hill and Drlll Seeder ........................... 18.00

No. 25 Iron Age Fertilizer Distributor Attaeliment...... 4.50

New Model Drill..................... 8.00 $4.00 \quad 3.50$ 5.00 4.00 11.00 8.50 8.00 6.00 8.50

14.00 3.60

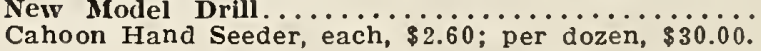

5.90

CULTIVATORS AND PLOWS.

No. 1 Iron Age Double Wheel Hoe, complete......... 7.00

No. 3 Iron Age Double wheel Hoe, with side hoes only.

No. 13 Iron Age Double Wheel Hoe, with side hoes and

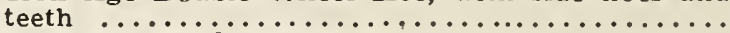

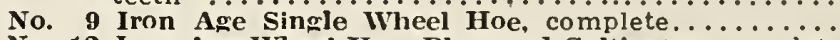

No. 12 Iron Age Wheel Ioe, Plow and Cultivator, complete

No. 19C Iron Age Wheel Plow and Cultivator...........

No. 20 Iron Age Single Wheel Hoe, complete...........

No. 21 Iron Age Single Wheel Hoe, plain (hoes only)....

Gell Cultivator. single wheel complete (lots of $6,10 \mathrm{c}$ less)

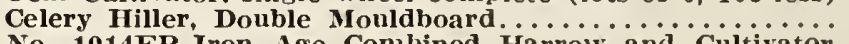

No. 1014EP Iron Age Combined Harrow and Cultivator,

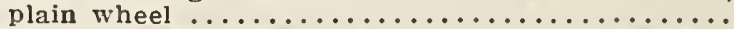

No. 600EL Iron Age Horse Hoe and Cultivator, less fur-

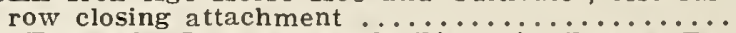
Extras for Iron Age Tools, $\dot{L}$ ist Priee Less 20 Per eent.

$4.25 \quad 3.65$

\section{$5.50 \quad 4.50$}

$5.25 \quad 4.25$

$3.50 \quad 3.00$

$3.50 \quad 3.00$

$6.00 \quad 4.70$

$4.00 \quad 3.20$

$5.00 \quad 4.00$

$16.00 \quad 14.00$

$6.90 \quad 5.00$

$8.60 \quad 6.00$

Net.

Aprons, Rubber, made of the best quality black sheeting. .\$1.80

Barrel Cart, Barrel and Truck with $21 / 2$-inch tires........11.50

Truck without barrel, but 1 pair trunnions....... 8.00

Bellows. American Sulphur, No, 0 , small................... 0.85

Bellows, American sulphur, No. 0 , small......................

No. 2, large................ 1.15

Jumbo Powder Guns..................... $\$ 25.00 \quad .20$

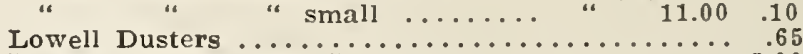

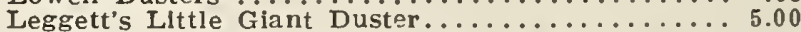

Books, Agrlcultural; 20 per cent. discount from publishers' prices.

Bonc Mills. See Poultry Supplles.

Doz.

$\$ 8.00$

10.00

11.00

2.25

1.10

6.75

Cape Flowers, Pure White. Extra fine quality . . . . . . . . . . .\$0.90

Carnation Bands. Tiny rubber bards, almost invisible, per box of

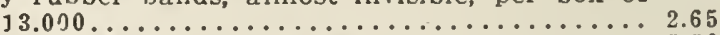

Supports. Common Seise. 2-ring, per 100 ; $1.75 ; 1000 \ldots 15.50$ F. o. b. New York, 2-ring, per 100, $\$ 2.25$; per $1000 \ldots 18.00$ 3 -ring, $100, \$ 2.25 ; 1000 \ldots \ldots \ldots \ldots \ldots \ldots \ldots \ldots \ldots \ldots . \ldots \ldots$ F. o. b. New York, 3-ring, per $100, \$ 2.75$; per $1000 \ldots 20.00$ 
Chenille Lettering, sllk, all colors...............\$ \$0.65 \$6.50

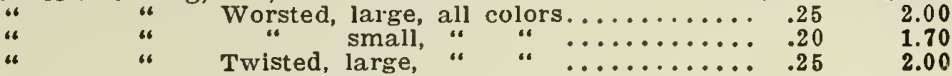

Clamps, Peerless repalr, box of $100,90 \mathrm{c}$.

Clips, Blake's Lever, per 1000, $\$ 1.35$.

Corn Planter, the Eagle..................................... 10

Dandelion Puller. The "Vaughan," made of malleable iroli and unbreakable. A splendid seller. Each, $30 \mathrm{c}$; doz., $\$ 3.25$; gross, $\$ 31.00$.

Dibber, Garden, Malleable. Per gross, $\$ 28.50$; per doz., $\$ 3.00$; each, $30 \mathrm{c}$.

Doves. Vaughan's Flexible. Extra quality, each, $\$ 1.20$; per doz., $\$ 12.00$. First quality, each, $\$ 1.00 ;$ doz., $\$ 11.00$.

\section{FERTILIZERS.}

\section{Subject to Market Changes. Terms, Net Caslr.}

All these fertilizers in bulk are f. o. b. Chicago. If shipped from New York, add 50c per $100 \mathrm{lbs}$, or $\$ 7.00$ per ton, except ltems marked whlch are either f. $o$. b. Chlcago or New York. If wanted In 25 or 50 lb. bags. $15 \mathrm{c}$ per 100 lbs, extra.

Acid Phosphate, 14 per cent available, 100 lbs., $\$ 1.35 ; 500$ lbs., \$5.25; 1,000 lbs., $\$ 10.00 ; 2,000$ lbs., $\$ 19.00$.

Vaughan's "Rose Grower" Bone Meal, 100 lbs., \$2.10; 200 lbs.,

$\$ 3.75 ; 500$ lbs., $\$ 8.75 ; 1,000$ lbs., $\$ 17.25 ; 1$ ton, $\$ 34.00$.

Bone Meal, 100 lbs, $\$ 1.80 ; 200$ lbs., $\$ 3.40 ; 500$ lbs., $\$ 8.00 ; 1,000$ lbs., $\$ 15.75$; ton, $\$ 31.00$.

Bone Flour, pure white, fine plant food, per 100 lbs., $\$ 2.35 ; 200$ lbs.,

$\$ 4.50 ; 500$ lbs., $\$ 10.50 ; 1000$ lbs., $\$ 20.00$; ton. $\$ 39.00$.

Raw Bone Meal, 100 lbs., $\$ 2.10 ; 200$ lbs., \$4.00; 500 lbs., $\$ 9.50$;

1000 lbs., $\$ 18.00$ : ton, $\$ 35.00$.

Bone Shavings, 100 lbs., $\$ 2.60 ; 200$ lbs., $\$ 5.00: 500$ lbs., $\$ 12.00$.

Bone and Blood, per 100 lbs., $\$ 1.65 ; 200$ lbs., $\$ 3.15 ; 500$ lbs., $\$ 7.50$; 1,000 lbs., $\$ 14.50$; ton, $\$ 28.00$.

Bone, Blood and Potash Fertilizer Mixture (analysis 5-8-3).

100 lbs., $\$ 2.40 ; 200$ lbs., $\$ 4.60 ; 500$ lbs., $\$ 11.00$; ton, $\$ 41.00$.

Cattle Manure, Shredded, 100 lbs., $\$ 1.25 ; 500$ lbs., $\$ 4.75 ; 1,000$ lbs.,

$\$ 8.50 ; 2,000$ lbs., $\$ 16.00$.

Clay's Fertilizer, Imported, 28 lbs., $\$ 2.00 ; 56$ lbs., $\$ 3.50 ; 112$ lbs., $\$ 6.00$.

If shipped from New York, $\$ 5.00$.

Dried Blood. 100 lbs.. $\$ 3.50 ; 200$ lbs., $\$ 6.90 ; 500$ lbs., $\$ 16.75 ; 1,000$

lbs., $\$ 33.00 ; 2,000$ lbs., $\$ 60.00$.

Fruit and Root Crop Manure, Fertilizer Mixture (analysis 2-8-3 per cent), 100 lbs., $\$ 1.70 ; 200$ lbs., $\$ 3.20 ; 500$ lbs., $\$ 7.50$; ton, $\$ 28.50$.

Horn Shavings, per 100 lbs., $\$ 3.75 ; 500$ lbs., $\$ 18.25$.

Hardwood Ashes, 100 lbs., $\$ 1.00 ; 200$ lbs., $\$ 1.95 ; 500$ lbs., $\$ 4.50$; ton. $\$ 17.00$.

* Ichthemic Guano, 112 lbs., $\$ 7.50$.

Kainit. Imported from Germany. Stopped account of war. Write us when in the market.

Land Plaster or Gypsum, 100 lbs., $65 \mathrm{c} ; 500$ lbs., $\$ 2.75$.

Muriate of Potash. Imported from Germany. Stopped account of war. Write us when in the market.

Nitrate of Soda, $100 \mathrm{lbs}$, $\$ 3 . \overline{0} 0$; original sack (220 lbs.), $\$ 7.25 ; 500$ lbs., $\$ 16.00 ; 1,000$ lbs., $\$ 30.50$.

Scotch Soot, 112 lbs., $\$ 3.50$.

Sheep Manure, Pulverized, per 100 lbs., $\$ 1.25 ; 500$ lbs., $\$ 5.00 ; 1,000$ lbs., $\$ 9.25$; ton, $\$ 18.00$. Subject to market change.

Sulphate of Potash. Imported from Germany. Stopped account of war. Write in when in the market.

Sulphate of Ammonia, 100 lbs., $\$ 6.00: 500$ lbs. $\$ 28.50$.

Thompson's Vine and Plant Manure, 112 lbs., \$5.00.

Thompson's Chrysanthemum Manure, $56 \mathrm{lhs,} \$ 50$.

Vaughan's $3-8-3$ Fertilizer, 100 lbs., $\$ 1.90 ; 200$ lbs., $\$ 3.60 ; 500$ lbs., $\$ 8.50 ; 1,000$ lbs., $\$ 16.50$; ton, $\$ 32.50$.

Vaughan's 5-8-3 Truck and Farm Manure, 100 lbs., \$2.35; 200 lls.. $\$ 4.50 ; 500$ lbs., $\$ 10.75 ; 1,000$ lbs., $\$ 21.00$; ton, $\$ 41.00$.

- Valsang, 112 lbs., $\$ 7.00$. 


\section{Vaughan's Lawn Fertilizer.}

The Best Lawn Dressing on the Market.

Odorless, lasting and effective. A good seller.

$100 \mathrm{lbs}, 1000 \mathrm{lbs}$.

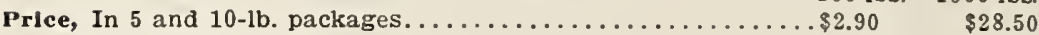

In 25 and $50-1$ b. bags......................... $2.45 \quad 21.50$

In $100-1$. bags............................... 18.50

Fertilizers in Packages for Rctailing.

Vaughan's Conc. P. Food, 1-lb. pkgs., box of 2 doz., \$3.20. Per doz., \$1.75 ................1/2-1b. pkgs., box of 2 doz.,\$1.80. Per doz.,\$1.00 Bowker's Plant Food.....1-lb. pkgs., box of 2 doz., $\$ 3.80$. Per doz., $\$ 2.00$ .... 1/2-lb. pkgs., box of 2 doz., $\$ 2.10$. Per doz., $\$ 1.10$ Flower Gatherer, nickle-plated............................ \$0.80

Flower Seed Gaugcs. These are made of brass and are very useful in putting up Flower Seeds. Set of 8 , per set............. \$1.50

Fumigator, Eureka, No. 1, capacity $1 / 2$ peck..............each.. 1.10 "Eureka, No. 2, capacity 1 peck............... .. 1.50

“ Eureka, No. 3, capacity 2 pecks............. " .. 2.10

“ Eureka, No. 4, capacity 3 pecks............... .. 2.80

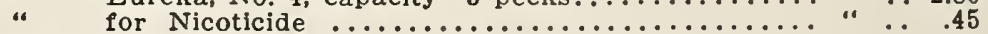

Garden Line, 1000 feet for $\$ 4.00$; per 100 feet...............45

Gardcn Line Reel, per doz., $\$ 5.50 \ldots \ldots \ldots \ldots \ldots \ldots \ldots \ldots \ldots$. . . . .50

Garden Roller. See Lawn Rollers.

Glass Cutter, Diamond Universal...................each . \$4.00

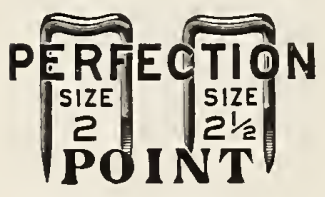

PERFECTION GLAZING POINT. ORIGINAL VAN REYPERS.

On orders for 100,000 and over, we supply cartons, with customer's name on same.

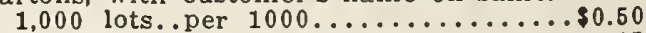

10,000 lots..per $1000 \ldots \ldots \ldots \ldots \ldots \ldots \ldots . .45$

100,000 lots..per $1000 \ldots \ldots \ldots \ldots \ldots \ldots . .35$

Write for prices on larger lots.

Siebert's Improved, $\%$ or $7 /$-inch long, lb., 35c; 5 Ibs., \$1.65;

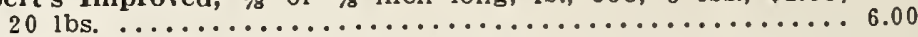

Glazing Tool and Glass Cutter, 3 doz., $\$ 1.35 \ldots \ldots \ldots \ldots \ldots$ per doz.. .50 Grafting Wax, $1 / 4$ lb. bars, per 1b., $25 \mathrm{c}$; 1/2 lb. bars, per 1b., $22 \mathrm{c}$;

$1 \mathrm{lb}$. bars, $20 \mathrm{c} ; 10 \mathrm{lbs}$. (1 lb. bars), per 1b., $19 \mathrm{c}$.

Grass Hools, English riveted backs, each, $40 \mathrm{c} \ldots \ldots \ldots \ldots \ldots$ doz. 4.25 Grass Collectors:

Philadelphia, Regular 12-inch, \$1.50; 14-inch, \$1.60; 16 -inch, $\$ 1.70 ; 18$-inch, $\$ 1.80 ; 20$-inch, $\$ 1.90 \ldots$.

Philadelplia, for style " $K$ " and High Wheel Mowers, 14 and $15-$ inch, $\$ 1.70 ; 16-17-$ inch, $\$ 1.80 ; 18-19-$
inch, $\$ 1.90 ; 20-21-$ inch, $\$ 2.00$.

Hoes, Scuffle, English, 6-inch, each, 40c; doz., $\$ 3.75 ; 8$-inch, each, $50 \mathrm{c}$; doz., $\$ 4.50 ; 10$-inch, each, 60c; doz., $\$ 5.50$.

Hose, "Electric," $3 / 4$ inch, 1 to 49 feet, $17 \mathrm{c} f t . ; ~ t 9$ to $99 \mathrm{ft} ., 16 \mathrm{c} \mathrm{ft} . ;$ 100 to 199 feet at $15 \frac{1}{2} \mathrm{c}$ foot; 200 feet and over at $15 \mathrm{c}$.

"Washers, rubber, for $3 / 4$-inch hose............per gross.. .25

"Clamps, $\$ / 4$-inch.........dozen, 40c; 3 dozen, \$1.10; gross.. 3.75

“Straps, $1 / 2-$ in. and $3 / 4$-in., $10 \mathrm{c} ;$ gr., $\$ 1.00 ; 1-i n .$, doz., $12 \mathrm{c} ;$ gr.. 1.30 


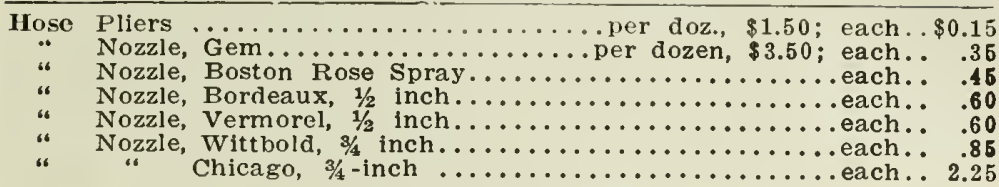

\section{SNAP HOSE COUPLER.}

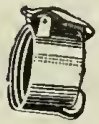

No.1

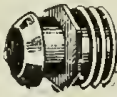

No.2

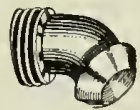

No.3

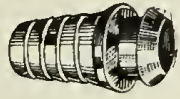

No. 4

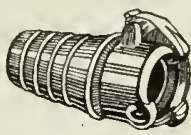

No. 5

Prices for $3 / 4$-inch Hose.

No. 1. Screws on to hydrant or old threaded coupling, use with No. 2 or No. 4 in connecting hose. Each, $18 \mathrm{c}$; dozen, $\$ 1.75$.

No. 2. Screws into faucet or ground pipe, on which it may be left per. manently, and to it the hose is attached by the lever connection, No. 1 or No. 5. Can also be screwed into old threaded coupling. Each, $12 \mathrm{c}$; dozen, $\$ 1.25$.

No. 3. Gooseneck, screws into the old threaded coupling. Most useful at the hydrant. Each, 12c; dozen, $\$ 1.10$.

No. 4. Corrugated, for insertion directly in the hose. Use with No. 5 for connecting hose. Each, 10c: doz., 85c.

No. 5. Corrugated, for insertion in the hose. Use with No. 4 for connecting hose. Each, 18c; dozen, \$2.00. Dozen Gros

IIoce Counler. Brass, $3 / 1$-inch ................... $\$ 0.90 \$ 10.00$

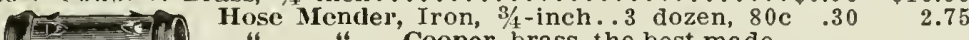
(1) $\quad 40$ " " Cooper, brass, the best made, $1 / 2$ and $3 / 4$-inch............ $65 \quad 7.25$ Cooper, brass, 1 -inch..... $85 \quad 8.50$

Hose IReducer, from 1 to $3 / 4$ inch.........each, $25 \mathrm{c} ; \mathrm{doz} ., 2.65$

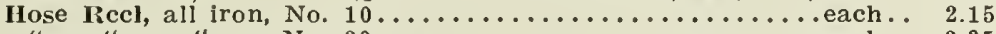

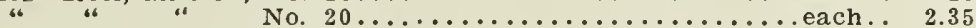

Hot Red Mats, Rattan Fibre, 6 feet by 6 fcet 2 inches......each.. 2.25

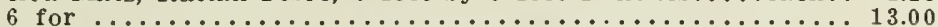

Iot Bed Mats, Burlap Lined, $40 \times 76$, each, $85 \mathrm{c}$; doz., $\$ 8.50$; $76 \times 76$, each, $\$ 1.35 ;$ doz., $\$ 13.50$.

Hot Bed Mats, Duck, $40 \times 76$, each, $\$ 1.25$; doz., $\$ 12.50: 76 \times 76$, each, Hyacintlı Glasses. $\$ 1.75 ;$ doz., $\$ 17.50$.

Tall Belgium, assorted colors. $\$ 1.25$ doz., $\$ 3.60$ for 3 doz., $\$ 13.50$ gross Tyes, assorted colors. $\$ 1.35$ doz., $\$ 3.75$ for 3 doz., $\$ 14.00$ gross.

Inmortelles, colored, subject to fluctuation....per 100 bunches.. 33.50 Per doz. ............................. 4.50 natural, subject to fluctuation.....

INSECTICIDES. Write for prices on large lots.

Int Exterminator. A non-poisonous powder. Sold in State of Illinois and New York only. Small box, 10c; doz., \$1.00;

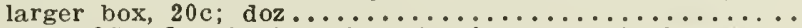

Arscnate of Lead, $1-1 \mathrm{~b}$. tins, $16 \mathrm{c} ; 5-1 \mathrm{~b}$. tin, $75 \mathrm{c} ; 25-1 \mathrm{~b}$. keg, $\$ 3.25$;

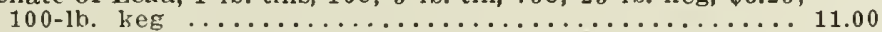

Aphine, 1 pt., $50 c ; 1$ quart, $90 c ; 1$ gallon............... 2.25

Aphis Punk, 1 pkg., 12 sheets, 55c; per case, 144 sheets......

Bordeaux Mixture, liquid, 1 -quart cans, $35 \mathrm{c}$ each; dozen, $\$ 3.70$; case $(36), \$ 10.40 ; 1$ gallon, $80 \mathrm{c}$; case $(12), \$ 8.75 ; 5$-gallon

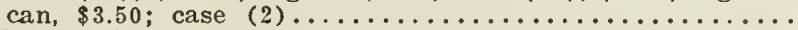

Bordeaux Mixture. powder, $1-\mathrm{lb}$. cartons, ea., $20 \mathrm{c}$; doz., $\$ 2.20$;

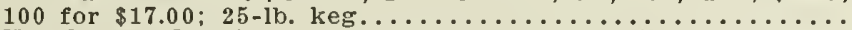
Eureka Weed Killer, $3-\mathrm{lb}$. tins, each, $75 \mathrm{c}$; doz., $\$ 8.50 ; 12 \mathrm{lb}$. tin,

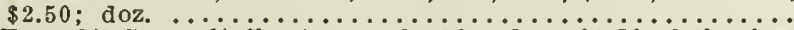

Fir Tree Cil Soap, $1 / 2-1 b$. tins, each, $23 \mathrm{c}$; doz., $\$ 2.50 ; 2-1 b$. tins, $65 \mathrm{c}$ each.

Flower of Sulphur (subject to fluctuation), 10 lbs., $40 \mathrm{c}$; (bbl.

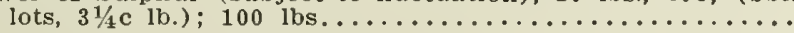


Fish Oil Soap, 1-lb. cartons, each, 13c; 25 for $\$ 3.00 ; 50$ for $\$ 5.50$; 1/2-1b. cartons; each, $7 \mathrm{c} ; 25$ for $\$ 1.65 ; 50$ for.

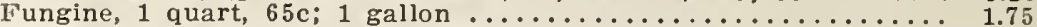

Horicum, per quart, $30 \mathrm{c}$; per gallon, $90 \mathrm{c}, 5$ gallons............ 4.00

Grape Dust, Hammond's, 5-1b. pkgs., $30 \mathrm{c}$; per 100 lbs., bulk....... 4.50

Hellebore, Powdered, 1-lb. pkgs., 30c each; case, 50 1-lb. pkgs.. 13.50

Kerosene Emulsion, 1-qt. cans, each, 35c; per doz., \$3.85; case

(36), $\$ 12.50$; gal. tin, 85c; case (12), $\$ 9.60 ; 5$ gals., $\$ 3.25$

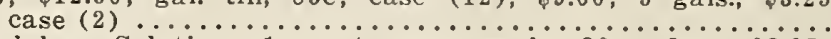

Lime Sulphur Solution, 1 -quart cans, each, $30 \mathrm{c}$; doz., $\$ 3.25$ case (36), $\$ 9.00 ; 1$ gal., $65 \mathrm{c}$; case $(12), \$ 7.25 ; 5$ gal.

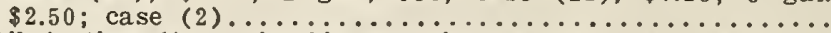

Lemon Oil, in tins, $1 / 2-$ pt. tin, $20 \mathrm{c}$ ea.; pint, ea., $35 \mathrm{c}$; per quart, $60 \mathrm{c}$; $1 / 2$ gal., $\$ 1.00$; per gal., $\$ 1.70 ; 5$ gal............

Nicoticide, 1 pint, $\$ 2.25$; $1 / 2$ pint, $\$ 1.15 ; 4$ ounces, $65 \mathrm{c}$; quart,

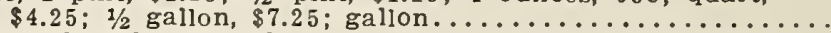

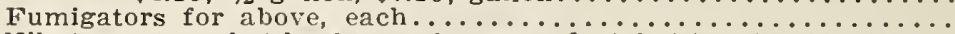

Nikoteen, 4 oz. bottle, $35 \mathrm{c}$ each; case of 48 bottles, $\$ 15.00 ; 1 / 2$

pt., $70 \mathrm{c}$; case, 10 bottles, $\$ 6.50$; per pt., $\$ 1.35$; case, 10 pts. 12.50

Nico-Fume, best and cheapest fumigating material, per tin 24

sheets, 75c; 144 sheets, $\$ 3.75 ; 288$ sheets..............

Nico-Fume Liquid, $1 / 1-1 b$. can, $45 \mathrm{c} ; 1-1 \mathrm{~b}$. can, $\$ 1.35 ; 4-1 \mathrm{~b}$. can

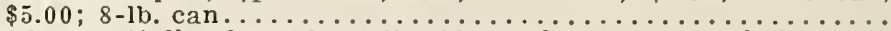

Paris Green, 1/2-lb. pkg., 16c; 1-lb., 28c; 5-lbs., \$1.25; 14-lb. keg

Slug Shot, in bulk, in bbls.. lnts $f$ o. b. cars....... per $100 \mathrm{lbs}$.

13.50

" "1-lb. cartons, each, $15 \mathrm{c} ;$ doz., $\$ 1.50$; case, $4 \mathrm{~S}, \$ 5.20$

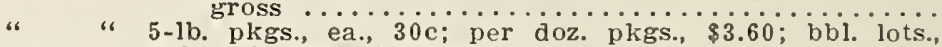

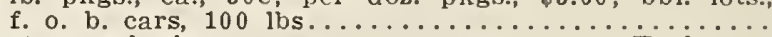

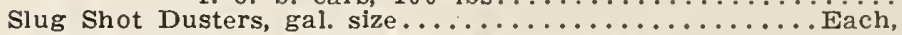

9.75

3.00

4.00

12.25

4.50

Solution of Copper, pint, $30 \mathrm{c}$; quart, $55 \mathrm{c}$; gal. ............ 1.60

Scalecide, qt., $45 \mathrm{c}$; gal., $\$ 1.00 ; 5$ gallons....................... 45

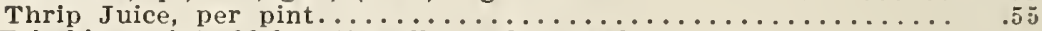

Tobakine, pint, $\$ 2.25$; $1 / 2$ gallon, $\$ 8.50$; gallon............. 16.00

Tobacco Soap, Sulpho, in 1/2-lb. pkgs., each, $15 \mathrm{c}$; per doz., $\$ 1.60$

3 dozen for $\$ 4.50$; in $2-o z$. pkgs., each, $7 \mathrm{c}$; per doz........

Tobacco Dust, regular grade, 1000 lbs., $\$ 18.00$; per $100 \mathrm{lbs} \ldots \ldots . .2 .50$

"fumigating and dusting, 100 lbs., $\$ 3.25 ; 1000$ lbs. 25.00

“ Stems, only in full bales of about 150 lbs., per 100 Ibs.,

$\$ 1.25$; per 1000 lbs., $\$ 9.00$ ( 3 bales $1000-1 \mathrm{~b}$. rate).

X. L. All liquid insecticide, f. o. b. Chicago, per 1/2 gal., $\$ 2.00$;

per gal., $\$ 3.75 ;$ f. o. b. New York, per $1 / 2$ gal., $\$ 1.50$; per gal...

\section{KNIVES.}

Sheffield English knives are the standard the world over. The fol-

lowing have been made specially for us. Try them and you will use no other:

Budding-Ivory handle, No. $112 \ldots \ldots \ldots \ldots \ldots \ldots \ldots$

Ivory Handle, 2 blades, No. $166 \% \ldots \ldots \ldots \ldots \ldots \ldots . \ldots \ldots$

Buffed blade, No. $186 \ldots \ldots \ldots \ldots \ldots \ldots \ldots .85$

German black handle, Ivory tip, No. $715 \mathrm{~B} \ldots . . . .80$

Ivory handle, No. $109 \ldots \ldots \ldots \ldots \ldots \ldots \ldots . . . \ldots \ldots$

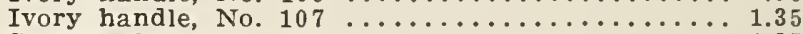

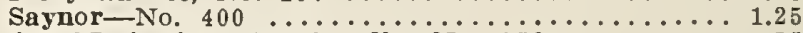

Pruning-Imitation stag handle, No. $052 \ldots \ldots \ldots . .55$

Genuine stag handle, No. $017 \ldots \ldots \ldots \ldots \ldots \ldots . . \ldots \ldots$

Genuine stag handle, 3 blades, No. $267 \ldots \ldots \ldots \ldots 1.10$

Genuine stag handle, 2 blades, No. $2671 / 2 \ldots . . . .9 .90$

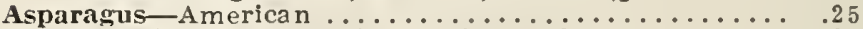

Beech handle $(3021 / 2)$, with Stationary blade........ 20

Propagating (109) English, Ivory handle......... 1.00

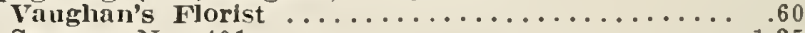

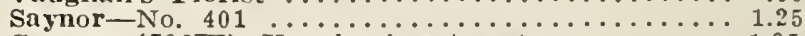

German (728T), Vaughan's extra strong........... 1.25

German ( $727 \mathrm{~W})$, white handle, brass caps, Budding

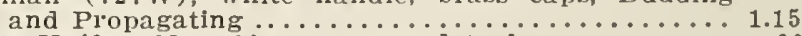

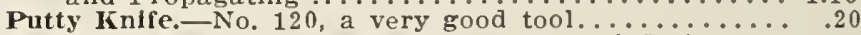

Doz.

$\$ 7.00$

13.50

8.50

7.50

9.00

13.50

14.50

5.50

12.50

11.00

9.00

2.75

2.25

11.00

6.25

14.50

12.50

Potato.-Humphrey's Concave, per gross, $\$ 15.00 \ldots \ldots$ 


\section{Philadelphia Lawn Mowers.}

\section{TERMS: NET CASH.}

\section{High Wheel (Steel), Style A.}

It has 10-inch driving wheels, 6 1/2-inch cylinder cutters with four knives.

\section{Width Weight List Price?}

15-inch... 40 lbs....\$25.00 Discount

17-inch...42 lbs....28.00 360 and 10

19 -inch...44 lbs....3 31.00
21 -inch...46 lbs....34.00

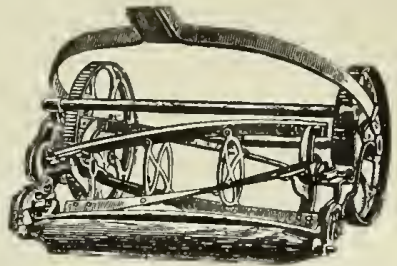

Style K.-10-inch, high wheel, 5 knife open wiper with patent corrugated blades.

Width Weight List Price

14 -inch . 40 lbs. $\$ 20.00$

16-inch..42 lbs..22.00

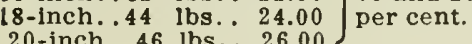

The Vaughan Mower.

Specially manufactured for our sale, high wheel, four-blade mower. Width Net Price Width Net Price 15 -in.....\$7.25 19-in....\$10.00 17 -in.....8 $8.00 \quad 21$-in.... 10.50

\section{Lawn Rollers, Inrham Water Weight} Less 30 WB-5 1 section, $18 \times 24,310 \mathrm{lbs}$. filler. List price... 13.00 percent WB-7 1 section, $24 \times 24,500 \mathrm{lbs}$. filler. List price... 17.00 Discount WB-9 1 section, $24 \times 32,650 \mathrm{lbs}$. filler. List price... 19.00

Crestlawn Mower.

W13-11 2 section, $18 \times 24,330 \mathrm{lbs}$. filler. List price.... 18.00

Strongest, easiest running mower built. Eleven-inch Ball Bearing drive wheels and automatic interlocking frame that cannot be knocked out of adjustme'st.

List price... 18.00

Lawn Horse Boots, The Link. Made in 3 sizes.

No. 2 for No. 2 horse shoe, $5 \frac{1 / 2}{2}$ in. inside measure.

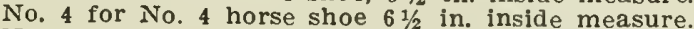

No. 6 for No. 6 horse shoe 7 in. inside measure.

List Price

Net

14 -in. $\ldots \ldots \$ 12.00 \ldots \ldots \ldots 8.50$

16 -in. $\ldots \ldots \ldots 13.00 \ldots \ldots .9 .50$

18 -in. . . . 14.00.... 11.00

20 -in. $\ldots \ldots \ldots 115.00 \ldots \ldots \ldots 11.50$

\section{POT AND TREE LABELS.}

Wo carry the best quality of Wooden Labels made in the country; they are smooth, strong and neat. Samples free.

Pot Mabels, Wooden,

In lots of 5000 we allow $5 \%$ discount.

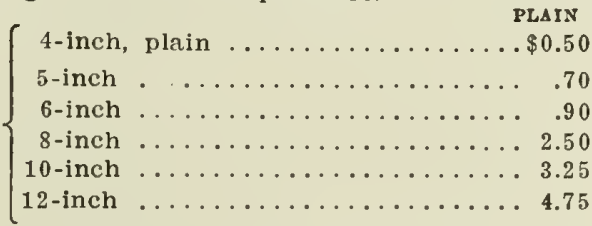

PAINTED $\$ 0.65$

Tree Labels, IVooden, $31 / 2$-inch, notched............ .55

Copper Labels, wired, indestructible. No. 1, per 100, $\$ 1.00$

....No. 2, per 100, 1.30

Lear Mold, for Ferns, etc....................................

Mastica, for glazing, 22 gals., $\$ 23.50 ; 10$ gals., $\$ 11.30 ; 6$ gals., $\$ 7.20$

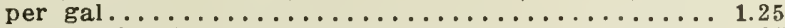

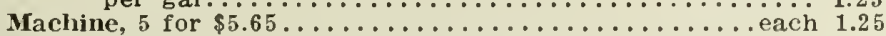

Match Stlcks, per 1000,5 -inch, $40 \mathrm{c} ; 12$-inch, $75 \mathrm{c} ; 18$-inch, $\$ 1.00$. Packages contain 250 sticks. We do not break packages. 
MATTING, Porto Rico. Used by all leading florists for decorating Azaleas, Hyacinths, Tulips, and in fact all blooming plants. They increase the sales of blooming plants.

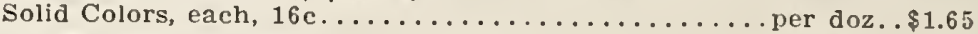

MOLE TRAPS, out o'Sight ......................... .70

.IOSS. (See also Sphagnum.)

French green, in square packages, pkg., 10c; per doz., $\$ 1.00$;

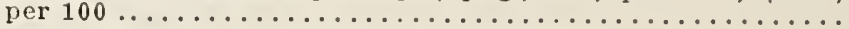

Sheet Moss. Per sack.......................... 1.35

Moss Wreaths. Write for Prices.

Paint Mount Beaeon Green, for shading greenhouses, gal., \$1.55;

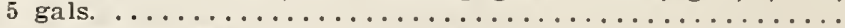

PAMPAS PLUMES. Natural. 32 to 36 inches. Per 1000, $\$ 23.50$;

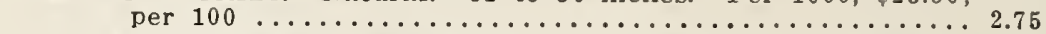

Paper, Manilla Tissue, 10 reams, $\$ 4.85 ; 400$ sheets, f. o. b. Chicago... .55 (480 sheets, $65 \mathrm{c} ; 10$ reams, $\$ 5.85$, f. o. b. New York.)

" Florists' White Tissue, per 4,000 sheets, $\$ 9.50 ; 400$ sheets

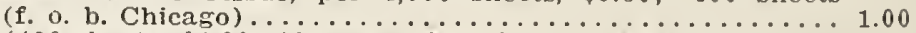
(480 sheets, $\$ 1.20 ; 10$ reams, $\$ 11.00$ f. o. b. New York.)

American Beauty Tissue, per ream, 60c; 10 reams (f. o. b.

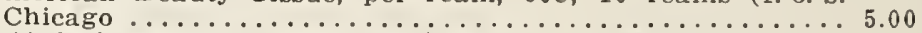
(480 sheets, $70 \mathrm{c} ; 10$ reams, $\$ 6.00$ f. o. b. New York.)

Manilla Wax, light, per 400 sheets, $\$ 1.30$; (480 sheets f. o. b.

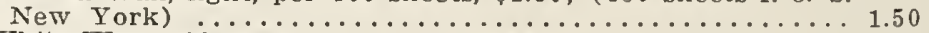

White Wax, 400 sheets (f. o. b. Chicago) ................... 1.40

“Green Wax, 400 sheets (f. o. b. Chicago)............. 1.75 (480 sheets f. o. b. New York, $\$ 2.00$ )

"Waterproof Crepe, per dozen rolls................ 2.25

Peat, Azalea, per bbl., F. O. B. Chicago................... 1.80

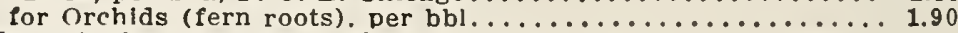

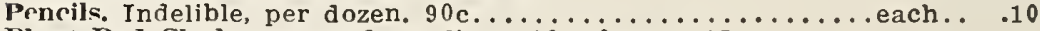

Plant Bed Cloth, per yard, medium, $10 \mathrm{c}$; heavy, $15 \mathrm{c}$.

We do not sell less than 20 yards of a kind.

\section{CANE STAKES.}

Standard Grade. From 6 to 8 feet long; welght, about 175 lbs. per 1000 . Price (if shipped from Chicago), per 100, 70c; 500 for $\$ 2.70$; per $1000 . \$ 4.80$.

Price (if shipped New Yorls), per $100, \$ 1.15 ; 500$ for $\$ 3.40$; per 1,000 , $\$ 6.50$.

Special price on larger lots on application.

Extra Ileavy Grade. For special work, Dahlias, etc. These run from ? to 12 feet in length and weigh about 360 pounds per 1000 .

Price (in Chicago only), per $100, \$ 1.45 ; 500$ for $\$ 6.50 ; 1,000, \$ 12.00$. Pipe Stems. For light work.

Price (from Chicago only), per 100,50 c; 500 for $\$ 1.75 ; 1000$ for $\$ 3.25$.

Japanese Bamboo Canes, light, strong and Aurable, per $100,60 \mathrm{c} ; 250$ for $\$ 1.35 ; 1000$ for $\$ 5.00 ; 5000$ for $\$ 23$.

Green Painted Japanese Bamboo. 2-ft., per $100,35 \mathrm{c}$; per $1000, \$ 3.15$; $21 / 2 \mathrm{ft}$., per $100,50 \mathrm{c}$; per $1000, \$ 4.25 ; 3-\mathrm{ft}$, per $100,60 \mathrm{c}$; per 1000 , $\$ 5.40 ; 4$-ft., per $100,70 \mathrm{c}$; per 1000 , \$6.25.

Plant Stakes, Wooden. Light turned, painted green, per 100,18 -inch, $80 \mathrm{c}$; 2 -ft., $\$ 1.55 ; 21 / 2-\mathrm{ft} ., \quad \$ 2.35 ; 3$-ft., $\$ 3.25 ; 31 / 2-\mathrm{ft} ., \$ 4.00 ; 4-\mathrm{ft} ., \$ 5.00 ;$ 5 -ft., \$5.75. Heavy, per 100.3 -ft., $\$ 4.90 ; 4$-ft., $\$ 6.25 ; 5$ ft., $\$ 8.00$.

Plant Stakes. Galvanized Steel Wire. Terms, net cash.

Price subject to advance without notice.

\begin{tabular}{|c|c|c|c|c|c|c|c|}
\hline O. 9 WIRE & PEN 100 & PER 1000 & No. 8 & & & PER 100 & PEB 10 \\
\hline ot & $\ldots \$ 0.30$ & $\$ 2$. & $31 / 4$ & foot & & $\$ 0.80$ & $\$ 7.2$ \\
\hline 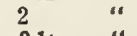 & .4 & 3. & 4 & " & $\cdots \cdots$ & 1.00 & 8 \\
\hline $2.1 / 2$ & $\ldots \ldots \ldots$ & $\begin{array}{l}4.50 \\
5.50\end{array}$ & 5 & “ & $\ldots \ldots \ldots$ & 1.10 & 10 \\
\hline
\end{tabular}


Plant Stand. Rolling, Fibcr.

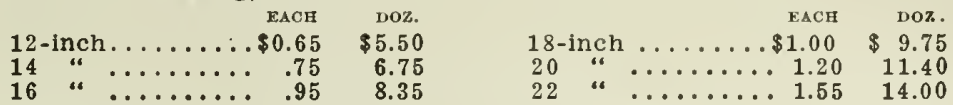

Pots. Neponset Flower. We are Sole Western Agents. F. O. B. Chicago, less 5 per cent in New York.

$21 / 4$-inch, packed 1000 to crate, per $100,25 \mathrm{c}$; per $1000, \$ 2.15$

$2 \frac{1}{2}$-inch, packed 1000 to crate, per $100,30 \mathrm{c}$; per $1000, \$ 2.50$

3-inch, packed 1000 to crate, per $100,40 \mathrm{c}$; per $1000, \$ 3.40$.

$31 / 2$-in., packed 1000 to crate, per $100,55 \mathrm{c}$; per $1000, \$ 4.85$.

4-inch, packed 500 to crate, per $100,75 \mathrm{c}$; per $1000, \$ 6.00$.

5 -inch, packed 500 to crate, per $100, \$ 1.15$; per $1000, \$ 9.75$.

6-inch, packed 500 to crate, pel $100, \$ 1.55$; per $1000, \$ 13.70$.

Pot Hangers, Kramer's.

per doz. $\$ 1.00$

FLOWER POTS, WHITE EARTHEN F. O. B. Chicago only.

\begin{tabular}{|c|c|c|c|c|c|c|c|}
\hline & & 100 & 1000 & & & 100 & 1000 \\
\hline 2 & inch & $\ldots \ldots \ldots \ldots \$ 0.50$ & $\$ 3.70$ & 5 & inch & $\ldots \$ 1.75$ & $\$ 14.00$ \\
\hline $\begin{array}{l}21 / 2 \\
3\end{array}$ & “ & $\begin{array}{cr}\ldots \ldots \ldots \ldots & .60 \\
\ldots \ldots \ldots \ldots \ldots & .70\end{array}$ & $\begin{array}{l}4.00 \\
5.50\end{array}$ & $\begin{array}{l}6 \\
7\end{array}$ & " & $\begin{array}{ll}\ldots & 2.50 \\
\ldots & 4.75\end{array}$ & 23.00 \\
\hline $31 / 2$ & “" & $\begin{array}{rr}\ldots \ldots \ldots \ldots \ldots & .80 \\
\ldots \ldots \ldots \ldots & .90\end{array}$ & $\begin{array}{l}6.50 \\
8.10\end{array}$ & $\begin{array}{r}8 \\
9 \\
10\end{array}$ & $\because$. & $\begin{array}{l}\ldots \ldots \cdots \\
\cdots \cdots \cdots\end{array}$ & \\
\hline 1 & “" & $\ldots \ldots \ldots \ldots 1.00$ & 9.50 & 12 & “ & $\ldots \ldots 22.00$ & \\
\hline
\end{tabular}

Fibre Saucers, superior to the old earthenware, light and not readily broken, easily cleaned.

BULB OR FERN PANS.

\begin{tabular}{rcrrr}
5 & inch. & & DOZ. & \multicolumn{1}{c}{100} \\
6 & “ & $\ldots$ & .45 & $\$ 2.00$ \\
7 & “ & $\ldots$ & .75 & 2.85 \\
8 & “ & $\ldots$ & .90 & 4.75 \\
10 & “ & $\ldots$ & 1.65 & 6.50 \\
12 & “. & $\ldots$ & 2.25 & 12.80 \\
& & & & 18.00
\end{tabular}

\begin{tabular}{|c|c|c|c|c|c|c|c|}
\hline \multirow{2}{*}{\multicolumn{2}{|c|}{ FBRE }} & & & \multicolumn{2}{|r|}{ FTBR } & \multicolumn{2}{|c|}{ E SAUCERS. } \\
\hline & & $\begin{array}{l}\text { SAUCERS } \\
\text { DOZ. }\end{array}$ & S. 100 & 9 & inch. & $\$ 1.90$ & $\$ 12.7$ \\
\hline & inch. & $\$ 1.30$ & $\$ 9.50$ & 10 & $"$ & 2.15 & 13.50 \\
\hline & “ & 1.40 & 9.75 & 12 & “ & 2.50 & 1 \\
\hline & “ & 1.55 & 10.75 & 14 & ") & 0 & \\
\hline & “" & 1.75 & 11.50 & 16 & “. & & \\
\hline & & & & & 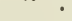 & 6.75 & \\
\hline
\end{tabular}

\section{RED FLOWER POTS.}

No. Pots in Crate.

Sold in crates only. F. O. B. Chicago. inch...1500..\$3.50 per 1000

$$
\begin{aligned}
& \text { “. . } 1000 \ldots 5.00 \text { " " } \\
& \text { “..500...7.75 } \\
& \text { “..250... } 1.00 \text { per } 100
\end{aligned}
$$

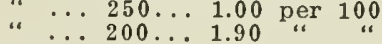

No. Pots in Crate.

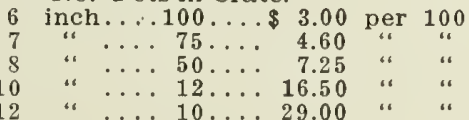

\section{PUMPS.}

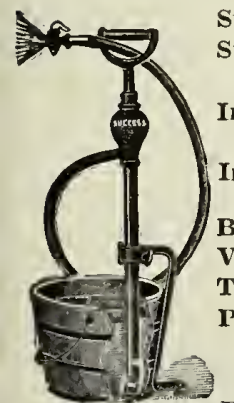

Success Spray Pump, best bucket pump made. . . . . 3.50

Sunshine Spray Pump, for orchard work complete with

2 leads hose........................ 8.25

Iron Age Vertical Barrcl Spray Pump No. 191, without barrel ............................... 1400

Iron Age, No. 190, 50 gal. horizontal, with single acting pump. without hose................. 17.50

Bordeaux Spray Nozzles, each 60c.; doz...........6.6.50

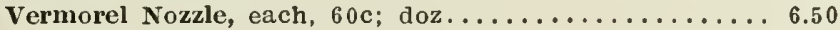

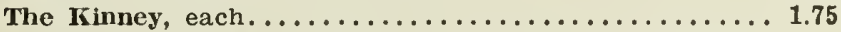

Putty, Twemlow's old English, semi-liquid, for glazing, in 1,2 and 3 gal. kits, per gallon, $\$ 1.50 ; 5$ and 10 gal. kits, per gal..................... 1.4 success PUMP Putty Bulb, Scollays, each, $80 \mathrm{c} ;$ doz. . . . . . . . . 8.75 


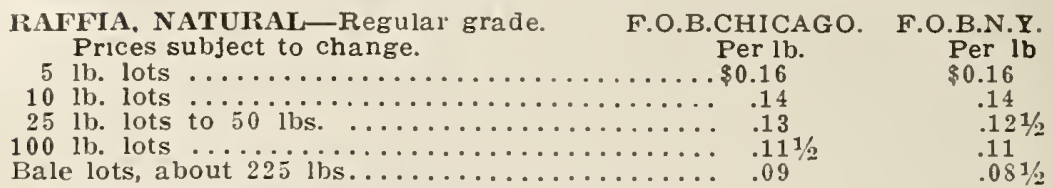

RAFFIA, NATURAL-Florist grade. F. O.B. Chicago. F. O. B. N. Y.

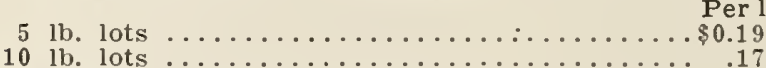

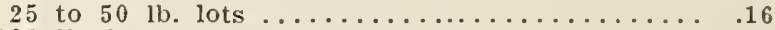

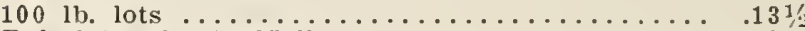

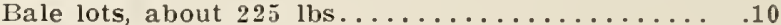

Per lb. $\$ 0.19$

.17

$.151 \%$

.13

$.091 / 2$

Per lb.

RAFTIA, COLORED.

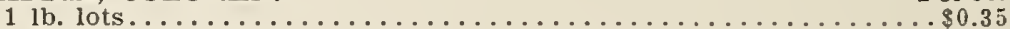

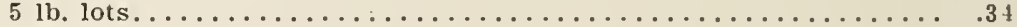

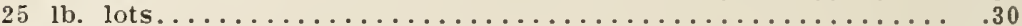

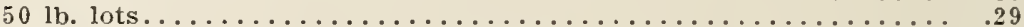

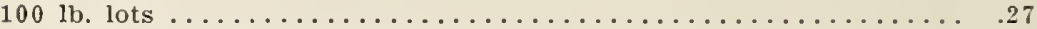

TERMS: First of montl following date of invoiec.

We do not open new accounts on this stock under \$25.00.

Please accompany order under that amount with casin.

\section{REEIS.}

The European war has stopped the importation of same from Gelmany and stocks are practically exhausted in the United States. Write us for list of stock and prices when in the market.

Splints, assorted colors, in 25 yard rolls, $1 / 4$ in. $w i d e, 15 \mathrm{c}$ each; $1 / 2$ in. wide, 25 c each.

Per oz. 1/4 lb. Per lb. 5 lbs.

Sweet Grass $\ldots \ldots \ldots \ldots \ldots \ldots \ldots \ldots \ldots \ldots \ldots . \$ 0.10 \quad \$ 0.25 \quad \$ 0.85$

Wood Ribbon .......................... . $85 \quad .25 \quad .90 \quad \$ 4.00$

\section{BOOKS ON BASKET MAKING, ETC.}

Indian Basketry.-By James. Cloth, 402 pages. Illustrated..... \$2.25 Raflia and Reed Weaving.-By E. S. Knapp. Cloth, 182 pages.

Flne for school work. Illustrated .................. .45

Basket Making.-By T. Vernette Morse. Paper, 32 pages. Richly

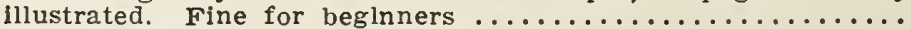

How to Make Baskets.-Thls is the title of a very linstructlve work by

Mary White. Bound in cloth, 194 pages. Iiistrated. Prlce... \$0.90

More Baskets and IIow to Make Them, by Mary White........... .90

Rake, Automatic, self-cleaning, 26 wood teeth...........each .. .60

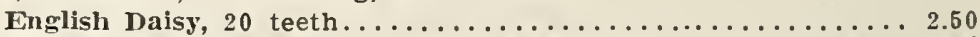

Lawn Queen, 24 wlre teeth, reverslble head, $30 \mathrm{c}$; Jumbo, 36 teeth. .60

Steel Bow, garden. Does not break in the center; 12 teeth, $50 \mathrm{c}$;

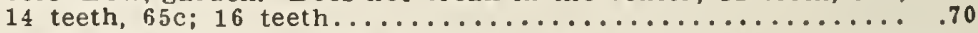

Sash. Hot Bed. No. 1 Cypress, $3 \times 6$ feet, unglazed, each, $\$ 1.35 ;$ doz., 15.50

$3 \mathrm{ft} .2$ in. by $6 \mathrm{ft}$., unglazed, each, $\$ 1.45 ; \mathrm{doz} \ldots \ldots \ldots \ldots \ldots \ldots . . . . .16 .50$

Sitw, Pruming, double edge, 16 -in., each, $70 c ;$ dozen.......... 7.50

$1 \mathrm{~S}$-in., each, soc; doz.. $\$ 8.50 ; 20$-in., each, $90 \mathrm{c}$; Doz. ........................ 9.00

Vaughan's Perfection. With adjustable blade. suitable for hand and pole sam, each, $\$ 1.25 ;$ doz., $\$ 14.50$. Extra

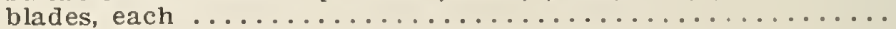


Scythe Bladcs. English; 30 inch, each, $\$ 1.10 ; 36$ inch, $\$ 1.20 ; 40$ inch 1.25

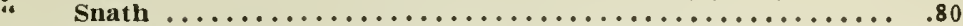

“ Stones. Talacre, imported, each, $15 \mathrm{c}$; per dozen......... 1.50

SEED BAGS. We furnish 250 and over of a slze at the 1000 rate, smaller quantlties are billed at an advance of 15 per cent. over 1000 rate.

These prices do not include printing.

Per 1000

Miniature, $2 \frac{1 / 2}{\times 1} 1 / 4$ inches, gummed, for Flower Seed......... $\$ 1.00$

Yellow, $2 \% / 8 \times 3 \%$ inches (No. 656), for Flower Seed, not gummed .90

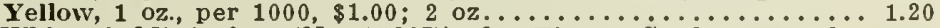

Whlte, $2 \times 3 \%$ inches (No. $1530 \mathrm{~B}$ ), for Flower Seed, gummed;

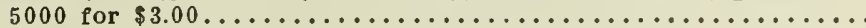

Extra IIeavy, per 1000,1 oz., $\$ 1.00 ; 2$ oz., $\$ 1.20 ; 4$ oz., $\$ 1.50$; $1 / 2$ pint, $\$ 1.80 ; 1$ pint, $\$ 2.50 ;$ quart, $\$ 2.75 ; 3$ pints, $\$ 3.75 ; 2$ quarts, $\$ 4.25 ; 3$ quarts, $\$ 5.25 ; 4$ quarts, $\$ 7.75 ; 6$ quarts,

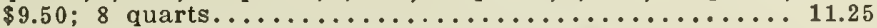

Seed Gauges, for Flower Seeds, brass, set of 8 , per set.............

" for Vegetable Seeds, tin, set of 4 , per set...............

\section{SHEARS.}

German Pruning.-Improved Spring, $4 \frac{1}{2}-$-inch, doz., $\$ 8.00$; each, 80c; $51 / 2$-inch, per doz., $\$ 9.75$; each, $90 \mathrm{c} ; 61 / 2$-inch, per doz., $\$ 10.50$; each, $\$ 1.00 ; 71 / 2$-inch, per doz., $\$ 11.25$; each, $\$ 1.05 ; 81 / 2-$ inch, per doz., $\$ 12.85$; each, $\$ 1.15 ; 91 / 2$-inch, per doz., $\$ 14.50$; each, $\$ 1.30$; extra springs for same, doz., $85 \mathrm{c} ; 10 \mathrm{c}$ each.

Buckeye Pruning, 20 inch handle, $\$ 1.25 ; 26$ inch handle..... 1.50

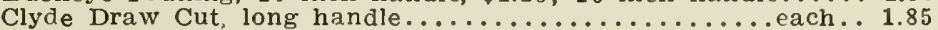
Sheep, with thumb guard, each, $30 \mathrm{c} \ldots \ldots \ldots \ldots \ldots \ldots$ per doz.. 3.25 Improved Wlre-Cutting, for Florist, per doz., $\$ 7.80 \ldots \ldots$ each $\ldots \quad .70$ Border English, long hand., 9-in. blade, ea., \$1,75, with wheel, 2.00 Grass, English, long handles with 2 wheels, 9 -inch......... 2.15 Hedge, English, 8-inch blade, $\$ 1.00 ; 9$-inch, $\$ 1.25 ; 10$-inch... 1.50 Sod Cutter, Richmond

SOD CIRUSHER NO. 1 MACHINE, total height, 3 1/2 feet; height of box, 18 inches; width, 20 inches; length, 24 inches. Price in New York. $\$ 13.50 ;$ In Chicago, $\$ 14.00$. No. 2 Machine, $\$ 18.00$.

Sphagnum Moss. Burlap bales, from Chicago, each, $\$ 1.00 ; 6$ bales for $\$ 5.00 ; 10$ for $\$ 9.50$. Write for prices on larger lots.

Wired bales, each, $\$ 1.00 ; 10$ for $\$ 9.00$.

Burlap bales, from Ncw York, each, $\$ 1.50 ; 3$ for $\$ 4.25 ; 10$ for $\$ 13.50$.

SPRAYERS.

Each

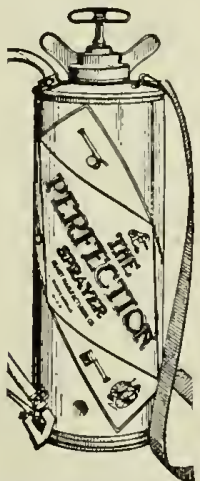

PERFECTION SPRAYER

Anto Spray, galvanized tank, stop cock....\$3.75

Auto Spray, galvanized tank and auto pop... 4.25

Iuto Spray, brass tank and stop cock.each. . 5.25

Iuto Spray, brass tank and auto pop.....6.6.00

Pcrfection Sprayer, brass tank........ 6.00

Perfection Sprayer, galvanized tank....... 4.50

The Lowell, glass tank, all tin, doz., $\$ 6.50 \ldots \quad .65$

Simplex Spray Pump, brass, $3 \frac{1}{2}$ gallon. complete, $\$ 5.50$; galvanized, complete. 3.50

Pncumatic, galvanized, 90c; brass...... 1.35

\section{SPRINKLERS.}

Merrill's Rotary or Butterfly, each, 25c; per doz. .................. 2.75 Evanston, each, $30 \mathrm{c} \ldots \ldots \ldots$..... per doz.. 3.25 Hartford, 2 foot, each, $65 \mathrm{c}$; doz., $\$ 6.50 ; 4$ foot, each, $75 \mathrm{c}$; per doz.. 7.80 

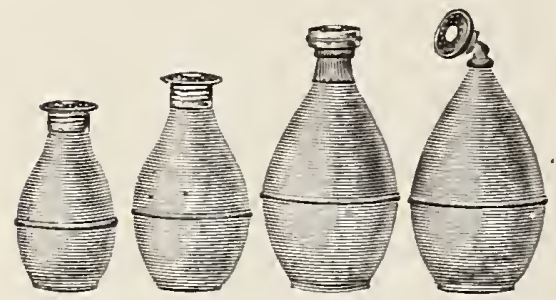

Scollay's Rubber.

None better for floral work. Beware of cheap imitations. We are sole Chicago Agents.

Each. Dozen.

Large size, No. 1..\$0.85 \$8.25

Medium size, No. $3 \quad .65 \quad 5.90$

Small size, No. 4.. $\quad .50 \quad 4.25$

Angle Neck large

size, No. $1 \ldots .$. .

.85

8.00

The Lenox.

Good seller; dozen. $\$ 3.50$

\section{SYRINGES}

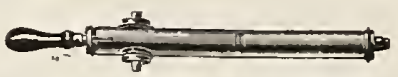

American, Brass, B, $\$ 2.15$; C, $\$ 3.25 ; \mathrm{D}, \$ 3.50 ;$ No. $5, \$ 5.50 ;$ No. $0 . \$ 1.75$

No. 12, English, Brass, with two sprays and 1 stream, 14-inch.. 2.50

No. 11, 18-inch, same style and size as No. $5 \ldots \ldots \ldots \ldots$. each . 3.75

Thermometer, Common, tin case, 8 -inch, 3 dozen, $\$ 3.25 \ldots$ per doz.. 1.15

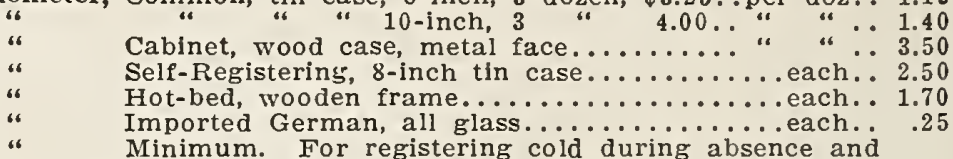

showing present temperature...........each.. 1.50

Tin Foil, 5 or 7 -inch, per 100 lbs. (net cash), subject to change.... 7.65

Tooth licks, per case of 100 boxes, including cartage (net cash) .. 4.50

Wired, per box of $10,000, \$ 1.60 ; 5$ boxes for ...... 7.00

Tobacco Stems. See "Insecticides."

Tonato Supports, heavy galvanized wire, per doz., $\$ 1.60$; per gross 18.00

Tree Pruner, Waters', each, 4-foot pole, 40c; 6 -foot pole........ .60

“ " 8-foot pole, 70c; 10 -foot pole, 80c; 12 -foot pole..... 90

" " extra blades, dozen, $\$ 1.25 \ldots \ldots \ldots \ldots \ldots \ldots \ldots \ldots$ each... .12

TERMS. First of month following date of invoice. We do not open new accounts on this stock under $\$ 25.00$.

Please accompany order under that amount with cash.

Trellises, Fan, per' dozen, 18-inch, \$1.35; 24-inch, \$2.00; 30-inch $\$ 2.85 ; 36$-in., $\$ 4.00 ; 31 / 2$-foot, $\$ 5.25 ; 4$-foot, $\$ 6.25 ; 5$-foot, $\$ 7.75$;

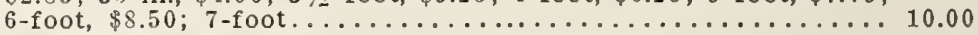

Veranda, per dozen, 5 -foot, $\$ 7.50 ; 6$-foot, $\$ 9.00 ; 7$-foot, $\$ 10.50$;

$\delta$-foot, $\$ 12.00 ; 9$-foot, $\$ 15.00 ; 10$-foot...................... 16.50

Trowels, Vaughan's Steel, the best, per dozen, $\$ 5.00 \ldots \ldots \ldots$ each. . .45

Trowcls, Garden, per dozen lots, 6 -inch, $65 \mathrm{c} \ldots \ldots \ldots \ldots$........... 7.00

English Steel, 6-inch, each, 1Sc; per dozen, \$1.75..gross.. 17.50

COLUMBIAN PLANT TUBS.

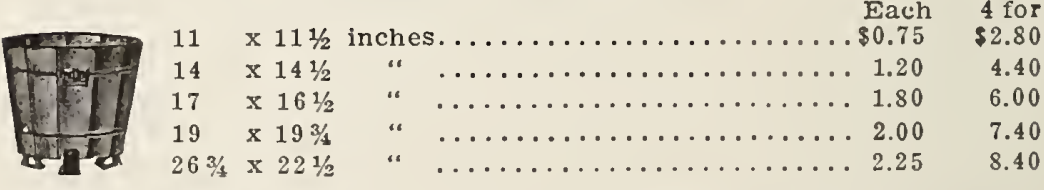

F. o. B. Chicago Each 4 for 2.80 


\section{UNION CYPRESS FIOUER TUBS.}

An excellent tub, cheap, neat and durable. Made from $7 / 8$-inch cypress, with iron handles and wooden feet, three iron hoops and two coats of green paint.

No. 1. $111 / 2$ inches....111/2 inches........ $\$ 0.90$

No. "2. $131 \%$ inches....111/4 inches............. 1.10

No. 3. $143 / 4$ inches....14 inches.......... 1.50

No. 4. 16 inches....15 inches.......... 1.80

No. 5. 19 inches....18 inches.......... 2.65

No. 6. 22 inches....191/4 inches......... 3.50

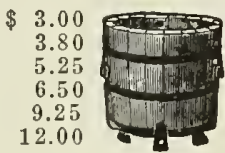

\section{Write for Discounts on Larger Lots.}

Turf Edger, round edge, per dozen, $\$ 4.75 ; 3$ for $\$ 1.25 \ldots \ldots \ldots$ each $\ldots \$ 0.45$

" " English half n:oon, without handle, dozen, $\$ 9.75$; each. . .90

TWEEZERS for Florists.-Imported.

4 -inch, each, 8c; per dozen..\$0.8 j 6-inch, each, $15 \mathrm{c}$; per dozen..\$1.40 5 -inch, each, $12 \mathrm{c}$; per dozen.. 1.35

\section{TIVINE.}

"The Queen" White Cotton. For tying parcels, flowers, etc. Put up in 5 -lb. sacks. Price.................... 1.40

Hemp. No. 18. For parcels, per 1b................ .2s

Linen, Red and white; best for training $\operatorname{Smilax}(4$ balls to lb.), 3-lb.

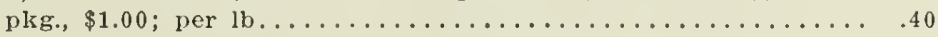

Green, for packages, very strong. Per $1 \mathrm{~b} \ldots \ldots \ldots \ldots \ldots \ldots . \quad .50$

Jute. For bunching, 2 or 3-ply; per 1b., $16 \mathrm{c} ; 10$ lbs., $\$ 1.50 ; 100$ lbs. 13.50

Silkaline. For stringing Snilax, etc.; fast green colors, will not fade or break.

FFF, Coarse, 2-oz. spools, 1-1b. in box, 8 spools........\$1.15

FF, Medium, " " " " " $" \ldots \ldots \ldots \ldots \ldots 1.15$

F, Fine, " $\quad$ " $\quad$ " $\quad$ " $\ldots \ldots \ldots \ldots \ldots 1.15$

Florists' Thread. Similar to Silkaline. Samples on application.

"King Arthur" brand, 2-oz. spools, 12 in a box............... .90

Florists' Glazed Fibre Vases. Indurated Fibre Florists' Vases for cutflower displays and storage purposes, cannot be excelled for richness. beauty and economy. They are too well-known to the Florist trade io require extended comment.

No. 1. Depth, 10 inches, diameter, $51 / 2$ inches $\ldots \ldots \ldots \ldots \ldots \ldots \$ 5.15$

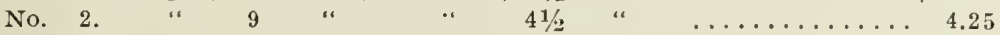

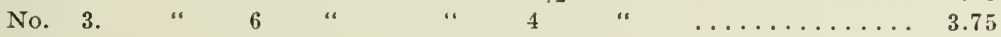

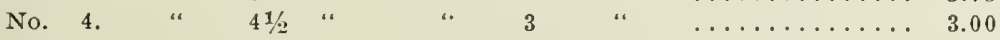

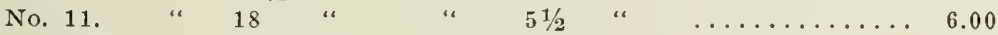

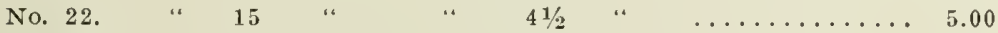

No. 33. " 12 " $12 \quad$ " $404 \ldots \ldots \ldots \ldots$

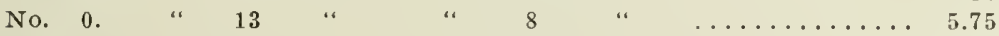

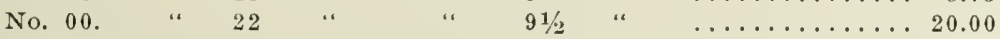

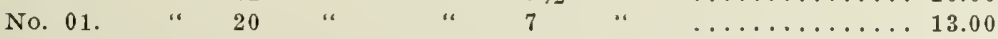

Vase. Clara Cemetery, eaeh, 20e; doz., \$2.25; per barrel of 3 doz., \$5.60. 
Watering Pots, American, gaivanized Iron, long spout, round, 2 copper faced roses with each pot. 4 quarts, each, $\$ 1.60 ; 6$ quarts, each, $\$ 1.70 ;$ s quarts, each, $\$ 1.90 ; 10$ quarts, cach, $\$ 2.25 ; 12$ qts. $\$ 2.50$ French (oval) style, same make; 6 quarts, $\$ 2.00 ; 8$ quarts, $\$ 2.25$;

10 quarts, $\$ 2.50 ; 12$ quarts ..................... 2.75

Wheelbarrow, The Vaughan Greenhouse.................. 4.00

"The Globe Garden ................... 3.50

\section{WEEDERS.}

Combination, per gross, $\$ 19.50 \ldots \ldots \ldots \ldots \ldots \ldots$ per dozen... 1.75

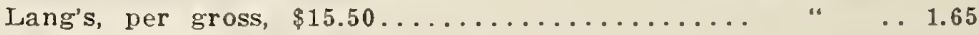

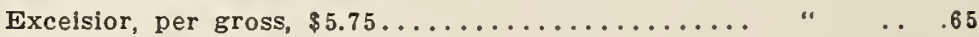

Hazeltine, per gross, $\$ 19.00 \ldots \ldots \ldots \ldots \ldots \ldots \ldots \ldots \ldots \ldots \ldots \ldots$. 1.75

Eureka, short handled, per doz., \$2.25; long handled “ . . 3.00

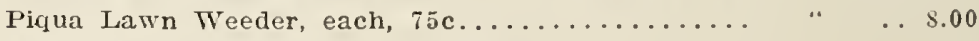

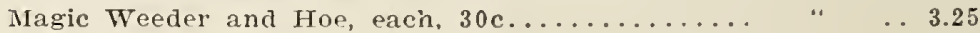

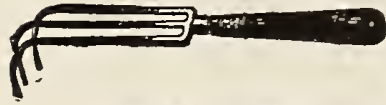

DUREKA WEEDER.

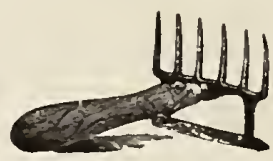

COMBINATION TFEDER.

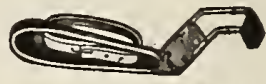

LANG'B WEEDER.

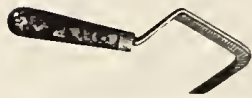

HAZELTINE WEEDER.

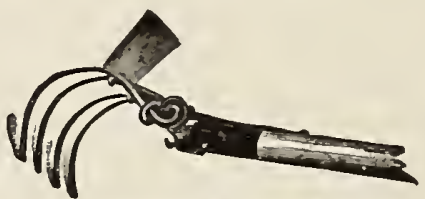

MAGIC WEEDER AND HOE.

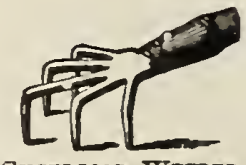

Exchlsion Wreper.

\section{FLORISTS' CUT WIRE.}

In Wooden Boxes.

The wire is prepared especiaily for Florists. Is ciean, extra soft white wire, stralghtened and cut in lengths ready for Immediate use, and in a convenlent form. 12 lbs. in each box, in lengths of 12 or 18 inches.

No. 20

$\$ 0.90$
22

$\$ 1.10$
24

$\$ 1.20$
26

$\$ 1.50$
28

$\$ 1.85$

\section{BRIGHT ANNEALED WIRE.}

Prices subject to change.

$12 \mathrm{jbs}$.

No. $18 \ldots \ldots \ldots \ldots \ldots \ldots \ldots .55$

No. $20 \ldots \ldots \ldots \ldots \ldots \ldots . . .60$

No. $22 \ldots \ldots \ldots \ldots \ldots \ldots \ldots \ldots . .70$

No. 24
No. 26

$12 \mathrm{lbs}$.

No. $28 \ldots \ldots \ldots \ldots \ldots \ldots \ldots \ldots . .95$

No. $32 \ldots \ldots \ldots \ldots \ldots \ldots \ldots 1.60$

No. $36 \ldots \ldots \ldots \ldots \ldots \ldots \ldots \ldots \ldots$ 


\section{TRADE LIST OF \\ POULTRY SUPPLIES.}

TERMS NET CASH.

We carry a complete line and shall be pleased to hear from you when in want of anything In thls line. We fill orders promptly and make prices as low as possible. We quote here some leading articles, description of these and other goods given fully in retall list. Write for prices on items not listed here. Prices, F. O. B., Chicago, except where noted.

\section{BONE CUTTF.RS.}

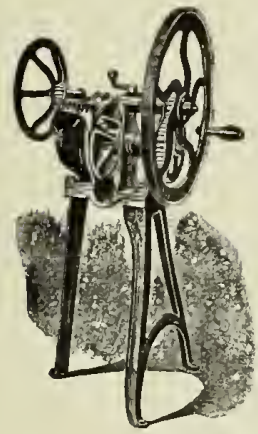

No. 1 HUMPEREY.

The Humplrey.-Simple, large capacity, easy to operate. The only open hopper green bone cutter that is also a practlcal vegetable cutter.

Retall Net.

No. 1. Hand power ..........\$12.00 \$10.75

No. 2. Small power ........... $13.50 \quad 12.10$

No. $21 / 2$ Hand and power......... $15.00 \quad 13.50$

Stover IIand Grinder. For grain bones, shells.

No. $32 \ldots \ldots \ldots \ldots \ldots \ldots \ldots \ldots \ldots \ldots \ldots \$ 4.00 \$ 3.60$

No. $33 \ldots \ldots \ldots \ldots \ldots \ldots \ldots \ldots \ldots \ldots \ldots .5 .00 \quad 4.50$

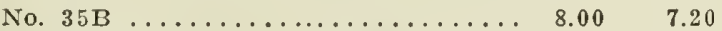

IVilson's "Crown" Bone cutter without stand ................. $6.50 \quad 6.00$

Wilson's "Crown" Bone Cutter with stand ................. $8.00 \quad 7.80$

No. 1. Bone Mill for crushing dry bones, shells, etc., without stand... $5.00 \quad 4.50$

No. 1. Bone mill with stand....... $7.00 \quad 6.30$

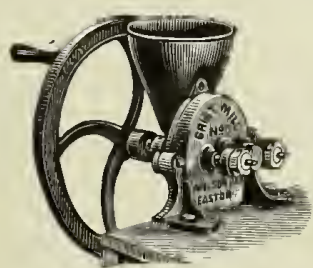

Wilson's Family Grist Mill for grinding and crushing all kinds of gralns.

No. 0. Family Grist Mill without stand ................. $5.00 \quad 4.50$

Humphrey's Rapid Clover Cutter.-Forty cuts of the knife to each revolution of hand wheel; easily operated.

Rapid Clover cutter with stand... $10.50 \quad 9.50$

NO. 0 FAMILY GRIST MILL.

\section{WHITEWASH AND SPRAY PUMPS.}

The $0-2$ IVhitewash Sprayer............... ret. $\$ 6.50 \ldots$ net. $\$ 5.40$ "Success" Bucket Sprayer, double acting, throwing continuous spray 3.50 The Lowell, glass tank, all tin, each $60 \mathrm{c} \ldots \ldots \ldots \ldots \ldots \ldots \ldots \ldots$ doz. 6.50 Junior Compress Air Sprayer (Galvanized) each $75 \mathrm{c} \ldots \ldots \ldots \ldots$ doz.. 8.10 Junior Compress Air Splayer (Brass) each $\$ 1.25 \ldots \ldots \ldots \ldots \ldots$ doz..13.50

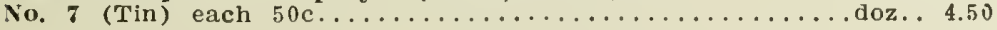




\section{PRAIRIE STATE INCUBATORS AND BROODERS.}

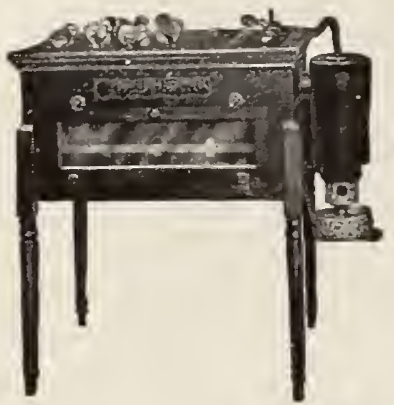

The Prairie State is the safest and most reliable Incubator a merchant can sell; every machine is guaranteed by the manufacturer and no risk whatever is assumed by the purchaser.

The Prairie State, because of its system of regulation and ventilation, is the most easily operated and the most certain in results of all incubators. It has done more to make poultry-raising profitable than all other machines combined. It is a machine upon which you r:an depend. It is the machine you want.

No. 0.-Diffusion, 100 hen eggs, $87 \mathrm{lbs}$. net weight; $135 \mathrm{lbs}$.

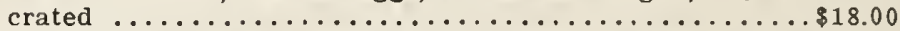

No. 1.-Diffusion, 150 hen eggs, 115 duck eggs-108 lbs. net weight; $160 \mathrm{lbs}$. crated.................. 22.50

No. 2.-Diffusion, 240 hen eggs, 200 duck eggs-175 lbs. net weight; $240 \mathrm{lbs}$. crated.......................... 32.00

No. 3.-Diffusion, 390 hen eggs, 300 duck eggs-220 Ibs.

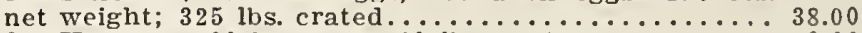

No. 0.-Keystone, 60 hen eggs, 60 ibs. crated........... 9.00

No. 1.-Keystone, 100 hen eggs, 80 lhs. crated.......... 12.00 WPICES OF COMBIN ATION COLONY BROODERS.

No. 1.- (Outdoor) Floor space $36 \times 72$ ins.- weight, crated,

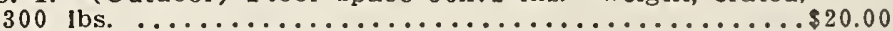

No. 2.-(Outdoor) Floor space $30 \times 60$ ins.-Weight, crated

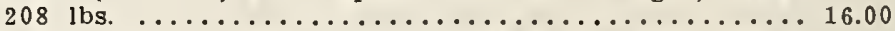

No. 3.-(Outdoor) Floor space $27 \times 48$ ins.- Welght, crated,

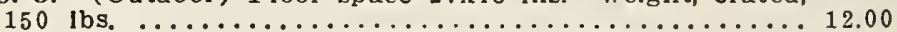

Net.

$\$ 16.26$

UNTVERSAI HOVER.

Hover, with lamp case, smoke conductor, lamp and ther-

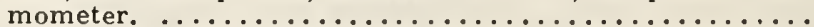

When used indoors alone, lamp case is not required.

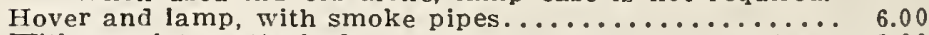

With regulator attached............................. 1.00 This hover is what we use in all our popular Colony brooders. be used in any style of box, brooder or colony house.

SPECIAL: Dealcrs' Orders will be Frcighted from Factory Prepaid.

Complete Pralrie State Catalogue with half-tone illustrations showing all different style Incuhators and brooders, actual dimensions and shipplng weights, mailed free unon application.

\section{VAUGHAN'S “ZENITH" HEN FEED.}

Vauglan's Zcnith Hen Fccl is different from nearly

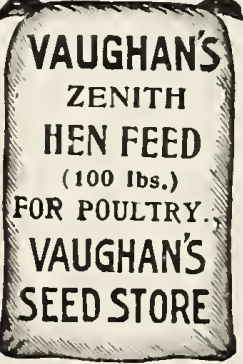
every hen food on the market. It contains no grit, no shell or other weight-making ingredients. Every ounce of "Zenith Fccd" Is an ounce of pure graln. The feed is composed of only sound, sweet grain, as we never, under any condition, allow any damaged grain to get Into this food. The mixture is made carefully and sclentifically. We belleve this Feed to be the cleanest and best balanced ration on the market today. Because Zenlth is an all-grain mixture, it is advisable in feeding to supply. in addition, Grit, Bone, Meat Meal, Charcoal and Green Food. If led In a litter of cut clover or hay, scratching is necessary and is an extremely beneficial exercise for the poultry. Samples and prices malled on application 


\section{VAUGHAN'S CHICK FOOD.}

This is a speclally prepared, balanced ration food for young chlcks whlch meets the full requlrements for growth, health and perfect development. There are two grades, designed for best results at all ages of the chick.

Fine-For chicks up to four weeks old.

Coarse-For chicks four weeks to four months old.

Samples and Prlces on Application.

Pigeon Food.- Put up in one grade only, as a whole food for young pigeons and a foundation food for large birds.

We put up Zenith Hen Food, Chick Feeds and Pigeon Food in handsome 5 -lb. bags to retail at $25 \mathrm{c}$; one dozen bags, $\$ 2.50$.

GRIT AND SHELLS.

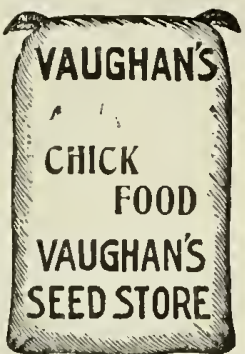

Per ton. 100 lbs.

Vaughan's Complete Pearl Grit, in 3 grades, Nos, 1, 2, 3. $\$ 12.00$

Foust's Iealth Grit for Pigeons. . . . . . . . . . . .

Crushed Oyster Shells.......................... 13.00

$\$ 0.65$

Cartage on Grlt and Shells, 25 c on less than 10 sacks.

\section{GROUND BONE.}

Granulated for young chicks, 25 lbs., $70 \mathrm{c} ; 50$ lbs., $\$ 1.20 \ldots \ldots \ldots \ldots \$ 2.25$

Coarse, for full grown fowl, 25 lbs., $70 \mathrm{c} ; 50 \mathrm{lbs}, \$ 1.20 \ldots \ldots \ldots \ldots 2.25$

\section{CHARCOAL.}

This alds in digestion and sweetens the contents of the crop.

Chlck and hen size, 25 lbs., $60 \mathrm{c} ; 50$ lbs., $\$ 1.00 \ldots \ldots \ldots \ldots \ldots \ldots \ldots \ldots 1 . \ldots \ldots$

\section{ALFALFA CLOVER MEAL.}

The best substitute for green grass.

Vaughan's Alfalfa Meal, 50-1b. bags, $\$ 1.10$.

\section{POULTRY FOODS.}

Doz.

Conkey's Laying Tonic, $25 \mathrm{c}$ size, each, $20 \mathrm{c} \ldots \ldots \ldots \ldots \ldots \ldots \ldots \ldots \ldots$

Conkey's Iaying Tonic, $50 \mathrm{c}$ slze, each, $40 \mathrm{c} \ldots \ldots \ldots \ldots \ldots \ldots \ldots \ldots \ldots$

Pratt's Poultry Food, $25 \mathrm{c}$ size, each, $20 \mathrm{c} \ldots \ldots \ldots \ldots \ldots \ldots \ldots \ldots \ldots . \ldots \ldots$

Pratt's Poultry Food, 50c size, each, $40 \mathrm{c} \ldots \ldots \ldots \ldots \ldots \ldots \ldots \ldots \ldots . \ldots . . . . \ldots$

Dried Blood Meal, 25, 50, or $100 \mathrm{lbs}$. Prices on application; subject to market changer.

Meat and Bone, 25, 50, or $100 \mathrm{lbs}$. Prices on application; subject to market changes.

Beef Scraps, 25, 50, or 100 lbs. Prices on application; subject to market changes.

\section{EGG PRODUCERS.}

Vaughan's "Zenith," $15 \mathrm{c}$ size, each, 12c................. 1.25

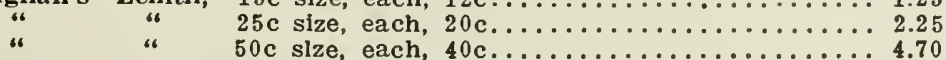

\section{BIRD SEED}

We llst the following: Canary, Hemp, Rape, Sunflower and Mixed. Prices on application, subject to market changes.

\section{STOCK FOODS.}

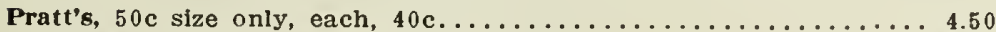

Conkey's Stock Vigor, $25 \mathrm{c}$ size, each, $20 \mathrm{c} \ldots \ldots \ldots \ldots \ldots \ldots \ldots \ldots \ldots \ldots .25$

Conliey's Stock Vigor, $50 \mathrm{c}$ size, each, $40 \mathrm{c} \ldots \ldots \ldots \ldots \ldots \ldots \ldots \ldots \ldots . \ldots \ldots$

\section{LICE POWDER.}

Sandford's, 16-oz. size, ea., 20c; doz., \$2.25. 40 oz. size, ea., 40c; doz. 4.25 100 oz. size, $85 \mathrm{c}$ each; doz..................... 9.50 


\section{LICE POWDERS—Continued.}

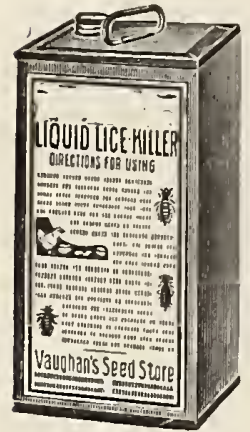

Conkey's 15 oz. size, each, $20 \mathrm{c} ;$ doz.......... 2.25

" 48 oz. size, each, $40 \mathrm{c} ; \mathrm{doz} \ldots \ldots \ldots \ldots .4 .50$

“ 100 oz. size, each, $80 \mathrm{c} ;$ doz......... 9.00

\section{LIQUID LICE KILLERS.}

Kice's 1 gal. size, eách, $75 \mathrm{c} ;$ doz.......... 9.00

“ 2 qt. size, each, $50 \mathrm{c} ;$ doz........... 5.50

“ 1 qt. size, each, $25 \mathrm{c} ;$ doz........... 2.75

Conkey's 1 gal. size, each, $75 \mathrm{c} ;$ doz. . . . . . . $\$ 8.30$

“ 2 qt. size, each, $50 \mathrm{c} ;$ doz.......... 5.75

1 qt. size, each, $30 \mathrm{c} ;$ doz.......... 3.50

Sulphur Candles, Conkey's $10 \mathrm{c}$ size, each $8 \mathrm{c}$; doz... $\quad .95$

\section{PIGEON AND POULTRY REMEDIES.}

Roup Cure, Vaughan's Good Health. Liquid, $50 \mathrm{c}$ size, each, $40 \mathrm{c}$; doz.\$4.25

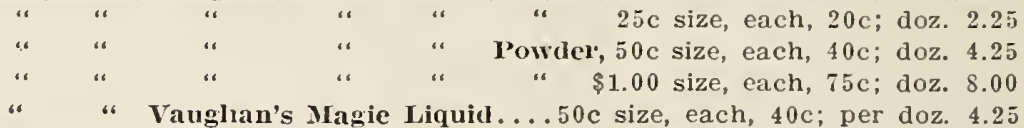

Roup Cure, Conkey ${ }^{\circ}$, $50 \mathrm{c}$ size, each, $40 \mathrm{c}$; doz., $\$ 4.00 ; \$ 1.00$ size, each,

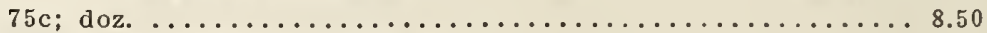

Vaughan's Good Healtl Tablets, per box, $40 \mathrm{c}$; doz. boxes........ 3.50

\section{LEG BANDS FOR POULTRY.}

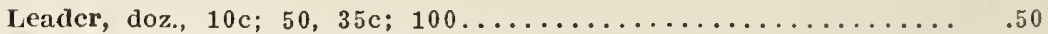

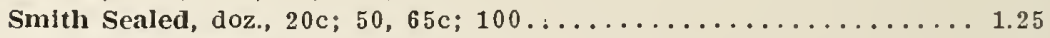

Improved Champion, doz. $10 \mathrm{c} ; 50,35 \mathrm{c} ; 100 \ldots \ldots \ldots \ldots \ldots \ldots \ldots . .50$

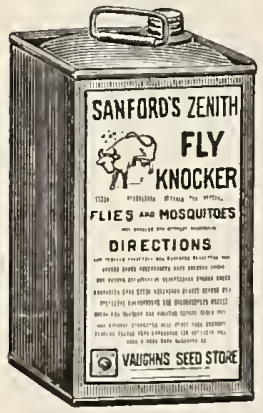

SANFORD'S “ZENITH" FLY KNOCKER.

An effective remedy against files and mosquitoes. also lice, screw worms and horn and blow flles.

It kilis lice on horses, cows and other animals.

Sandford's 1 gal. size, each...........\$1.00

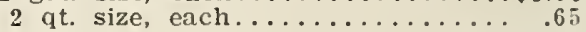

1 qt. size, each..............40

MILK OIL LIQUID DISINFECTANT

As a general disinfectant and eleaner stands without an equal. Infallible destroyer of all vermin and disease germs. Certain eradientor of inseets and disease of all animals. If ills Cockroaches, Water Bugs, Ants, etc.

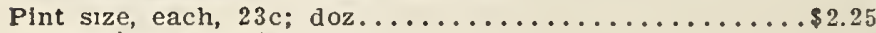

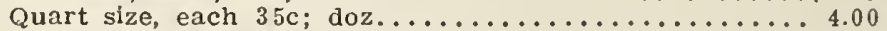

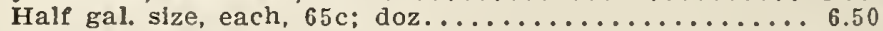

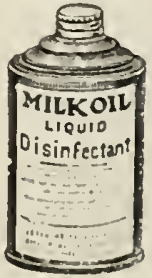

We carry sevcral other Lice Killing l'owders and Liquids, Disinfectants and Poultry Remedies. We are headquarters for Bird Seed of all kinds. 


\section{DRINKING FOUNTAINS}

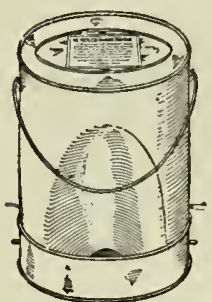

PEERLESS. FUUNTAIN.

Moc's Wall Fountain, No. 25, about $1 / 2 \mathrm{gal} \ldots \$ .30 \$ 3.50$

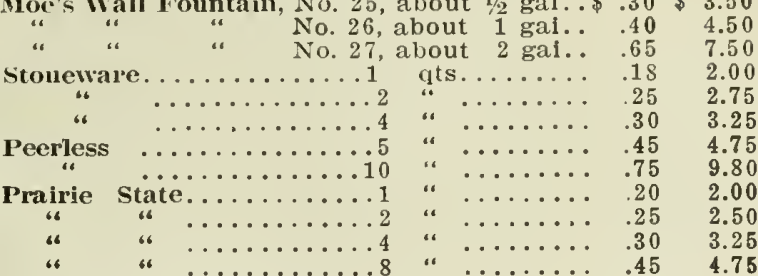

Moe's Top-Fill Fountaiu... g. gai......... 1.10

" "

" "

Brooder Fonntain...1 qt........ .25

2.50

\section{SHIPPING COOPS.}

All-Wood Shipping Coops. No. 1, each, 30c; doz., \$3.00. No. 2, each, 35c; doz., \$3.50. No. 3 , each, $35 \mathrm{c}$; doz., \$3.50. No. 4 each, $40 \mathrm{c} ;$ doz., $\$ 4.25$. No. 5, each, 50c; doz., \$5.25. No. 6 each, 55c; doz., \$5.75.

Vaughan's Exhibition Coop. Trio size, each, $\$ 2.00$; pen size, each $\ldots \$ 2.25$

\section{MISCELLANEOUS.}

Petty Poultry Punch, each, 18c; doz......\$2.00

Spring Lever Punch, each, 18c; per doz..... 2.00

Poultry Killing Knife (Piling), each, $40 \mathrm{c}$; doz.4.50

Egg Carriers, Reilabie. 12 doz., ea., $40 \mathrm{c}$; doz. 4.50 Ontario, 1 doz., 50 for $40 \mathrm{c}, 1,000$

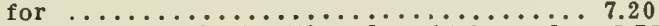

Egg Boxes, Eyrie, 1 setting, doz., $\$ 1.20 ; 5$ doz. 5.75

Egg Boxcs, Eyrie, 2 setting, doz., $\$ 1.65 ; 5$ doz. 8.00

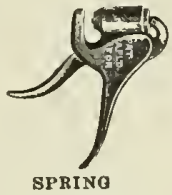

SPRING

Grit Boxes, (Vaughan's wooden), each, 35c; per doz.....\$3.40

" "Sanitary, (Gaivanized), each, $45 \mathrm{c}$; per doz..5.00

Moe's Never Clog and Waste Proof Hopper. This hopper is constructed on a principle hitherto unused. Does away with ail trouble of feed clogging no matter how coarse. The gradual enlargement to the base together with the vibration of the fowis feeding from the trough will force the feed downward. It is a great feed saver. Every safeguard has been piaced in the construction of the trough to prevent the birds from throwing out the feed. A trial wili convince you that it is a hopper of the greatest merit. Manufactured of heavy galvanized iron in three sizes.

No. $35-81 / 2$ inches wide, 12 quarts capacity....... EACH. $\$$ DOz.

No. 36 - 12 inches wide, 18 quarts capacity......... $.90 \quad 10.00$

No. $37-24$ inches wide, 36 quarts capacity ........ $1.35 \quad 15.00$

Nest Eggs, porcelain, doz., $20 \mathrm{c}$; gross................... 2.00

" $"$ Rust's Medicated, doz., $50 \mathrm{c}$; per gross ............... 5.50

Pigeon Nests, (Earthenware), per doz., $\$ 1.10$; per $100 \ldots \ldots \ldots \ldots \ldots . . .80$

" (Fiber), per doz., $\$ 1.10$; per $100 \ldots \ldots \ldots \ldots \ldots \ldots \ldots . \ldots . . \ldots$

“Perches, Muir's Iron, per doz., $85 \mathrm{c}$; per $100 \ldots \ldots \ldots \ldots \ldots \ldots 6.50$

" " Jersey, per doz., 60c; per $100 \ldots \ldots \ldots \ldots \ldots \ldots \ldots \ldots 4.75$

Caponizing Sets, complete, with book of instructions........... 2.00

Wire Hens' Nests, each, $13 \mathrm{c} ; \mathrm{doz} \ldots \ldots \ldots \ldots \ldots \ldots \ldots \ldots \ldots \ldots \ldots \ldots$

Salt Cat for Pigeons. Sait Cat is a scientific combination of those aromatic and tonic properties contained in such seeds and roots as gentian, anise, coriander, etc., combinet with sulphur, charcoal, limestone, sait, bone and other materials that are generaliy recognized by pigeon keepers as aiding digestion, promoting good heaith and egg produoton in pigeons and game fowls. Price, per doz. brlcks,...... \$1.60 


\section{VAUGHaN's Parks LaWn SeEd}

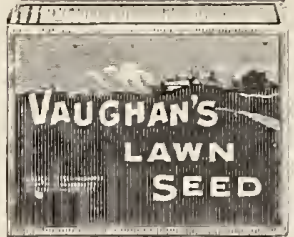

2 lb. box.

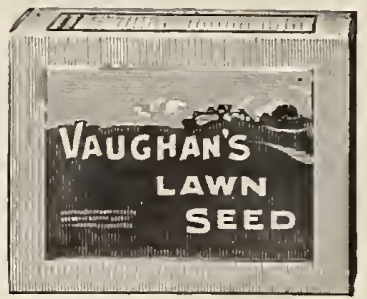

5 10. Dux,

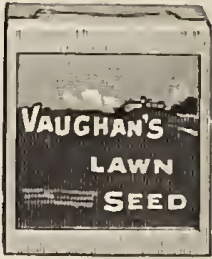

1 Ib. box.

\section{VAUGHAN'S LAWN GRASS SEED.}

I dealer selling Vaughan's Lawn Grass Mixtures is assured that there are no better grades sold at their respeetive priees. Vaughan's Chicago Parks, Central Park and Columbian Mixtures are the highest grade possible to make. There is no brand so well or favorably known, and our many year's of extensive advertising make Vaughan's Lawn Grass Seed a sure and ready seller.

Grass Seed is high again this year, due to the drought the past season. We purehased early from the eream of the American erops, and our representative whom we had in Europe all of this fall bought the best European grasses, samples absolutely impossible to duplicate at this time. An equal quality mixture cannot be sold for less money.

\section{VAUGHAN'S “CHICAGO PARKS" Highest Grade}

Paekage Quantity Priees.

Half pound (sell for $20 \mathrm{c}$ each), per $100 \ldots \ldots \ldots \ldots \ldots \ldots 10.00$

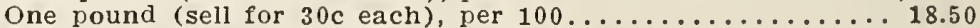

Five pounds (sell for $\$ 1.35$ eaeh), per doz............. 11.60

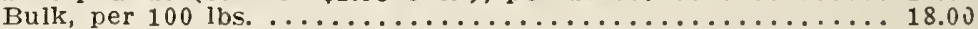

OUR OTHER STANDARD MTXURES.

COLUMBIAN "SHADY NOOK" LAIVN GRASS MIXTURE, for shaded places, under trees, ete. Price same as Chicag , Parks.

"QUICK SIIOIV" LAIVN SEED, for immediate effect. 10 lbs.,

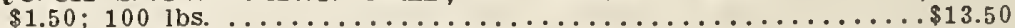

“GOOD MIXED" LAIVN GRASS SEED. $10 \mathrm{lbs} ., \$ 1.50 ; 100 \mathrm{lbs} . \ldots .13 .50$ KENTUCKY BLUE GRASS. Faney Clean.

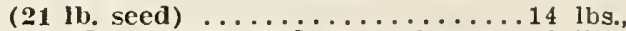

RED TOP, Fancy Seed. Extra heavy..10 lbs., Unhulled ................ 10 lbs.,

$\begin{array}{rr}\$ 1.85 & \$ 100 \text { lbs. } \\ 2.75 & 25.00 \\ 1.50 & 12.00\end{array}\left\{\begin{array}{l}\text { Subjeet to } \\ \text { ehange } \\ \text { without } \\ \text { notice. }\end{array}\right.$

$\begin{array}{ll}2.75 & 25.00 \\ 1.50 & 12.00\end{array}$ wotice.

CLOVER, DUTCH WHITE. Very fine quality. Write for prlees. Terms on al' Grasses or Clovers striclly cash.

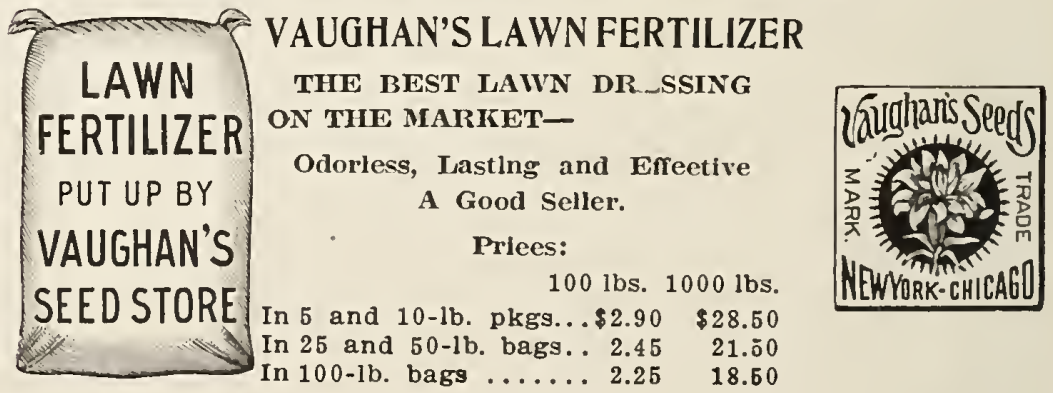




\section{CONTRACT \\ $S A V E$ \\ MONEY}

TAKE ADVANTAGE of our advance growing contracts on the items below by sending us your order in February or March. Let us quote you on your needs.

ONION SETS

BEANS

\section{SWEET CORN}

\section{GLADIOLUS}

\section{TUBEROSES}

\section{CALADIUMS}

Our growers are the pick of the Chicago onion set sections.

Our own growing, from seed stocks bred on our Michigan Farms.

Extra fancy, strains of Early sorts, (Michigan grown).

Grown on our three farms from our own stocks. We sterilize planting stock.

Cured and packed under the supervision of our own representative. 


\section{Vaughan's Lawn Seed}

Of Highest Purity and Germination

Home-grown and Direct Importations

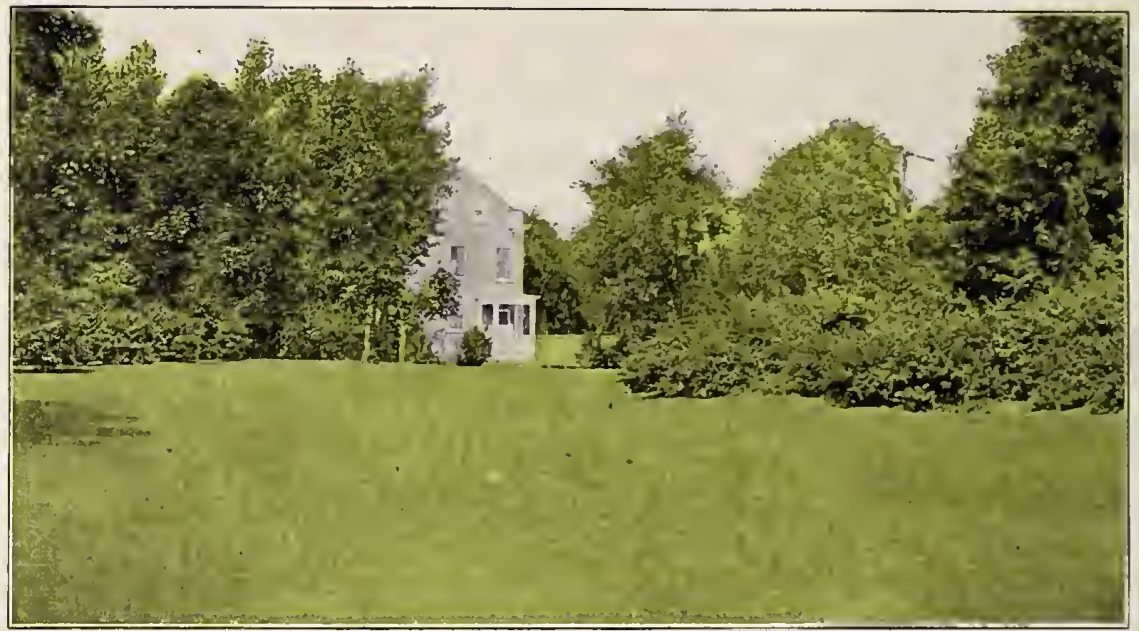

SPECIAL MIXTURES FOR

Lawns, Golf Courses, Tennis Courts, Etc.

Vaughan's "CHICAGO PARKS" and "CENTRAL PARK" Mixtures have been and are so thoroughly advertised that they sell themselves. This is the kind it will pay you to handle. 